\title{
Measurements of Molecular Diffusion Coefficients of \\ Carbon Dioxide, Methane and Propane in Heavy Oil \\ Under Reservoir Conditions
}

A Thesis

Submitted to the Faculty of Graduate Studies and Research

In Partial Fulfillment of the Requirements for the

Degree of Master of Applied Science in

Petroleum Systems Engineering

University of Regina

by

Asok Kumar Tharanivasan

Regina, Saskatchewan

December 2004

(C) Copyright 2004: Asok Kumar Tharanivasan 


$\begin{array}{ll}\begin{array}{l}\text { Library and } \\ \text { Archives Canada }\end{array} & \begin{array}{l}\text { Bibliothèque et } \\ \text { Archives Canada }\end{array} \\ \begin{array}{l}\text { Published Heritage } \\ \text { Branch }\end{array} & \begin{array}{l}\text { Direction du } \\ \text { Patrimoine de l'édition }\end{array} \\ \begin{array}{l}\text { 395 Wellington Street } \\ \text { Ottawa ON K1A ON4 }\end{array} & \begin{array}{l}\text { 395, rue Wellington } \\ \text { Ottawa ON K1A ON4 } \\ \text { Canada }\end{array}\end{array}$

Your file Votre référence

ISBN: 0-494-04009-2

Ourfile Notre référence

ISBN: 0-494-04009-2

NOTICE:

The author has granted a nonexclusive license allowing Library and Archives Canada to reproduce, publish, archive, preserve, conserve, communicate to the public by telecommunication or on the Internet, loan, distribute and sell theses worldwide, for commercial or noncommercial purposes, in microform, paper, electronic and/or any other formats.

The author retains copyright ownership and moral rights in this thesis. Neither the thesis nor substantial extracts from it may be printed or otherwise reproduced without the author's permission.
AVIS:

L'auteur a accordé une licence non exclusive permettant à la Bibliothèque et Archives Canada de reproduire, publier, archiver, sauvegarder, conserver, transmettre au public par télécommunication ou par l'Internet, prêter, distribuer et vendre des thèses partout dans le monde, à des fins commerciales ou autres, sur support microforme, papier, électronique et/ou autres formats.

L'auteur conserve la propriété du droit d'auteur et des droits moraux qui protège cette thèse. $\mathrm{Ni}$ la thèse ni des extraits substantiels de celle-ci ne doivent être imprimés ou autrement reproduits sans son autorisation.
In compliance with the Canadian

Privacy Act some supporting forms may have been removed from this thesis.

While these forms may be included in the document page count, their removal does not represent any loss of content from the thesis.
Conformément à la loi canadienne sur la protection de la vie privée, quelques formulaires secondaires ont été enlevés de cette thèse.

Bien que ces formulaires aient inclus dans la pagination, il n'y aura aucun contenu manquant.

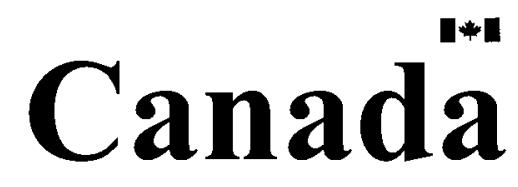




\section{UNIVERSITY OF REGINA}

\section{FACULTY OF GRADUATE STUDIES AND RESEARCH}

\section{SUPERVISORY AND EXAMINING COMMITTEE}

Asok Kumar Tharanivasan, candidate for the degree of Master of Applied Science, has presented a thesis titled, Measurements of Molecular Diffusion Coefficients of Carbon Dioxide, Methane and Propane in Heavy Oil Under Reservoir Conditions, in an oral examination held on December 8, 2004. The following committee members have found the thesis acceptable in form and content, and that the candidate demonstrated satisfactory knowledge of the subject material.

External Examiner: $\quad$ Dr. Yee-Chung Jin, Faculty of Engineering

Supervisor: Dr. Yongan (Peter) Gu, Faculty of Engineering

Committee Member: $\quad$ Dr. Ezeddin Shirif, Faculty of Engineering

Committee Member: $\quad$ Dr. Gang (Gary) Zhao, Faculty of Engineering

Chair of Defense: $\quad$ Dr. Liming Dai, Faculty of Engineering 


\begin{abstract}
Design and modeling of solvent-based heavy oil recovery processes require the knowledge of solvent diffusivity in heavy oil. In this thesis study, a non-intrusive pressure decay method is applied to measure the molecular diffusion coefficients of three different gaseous solvents, carbon dioxide, methane and propane, in heavy oil under reservoir pressure and temperature conditions. In the experiment, direct physical contact of the solvent and the heavy oil is made and thereby the pressure in the solvent phase versus time data are accurately measured inside a closed high-pressure diffusion cell at constant temperature, as the solvent gradually dissolves in heavy oil. On the other hand, in terms of the conservation of mass and the equation of state for a real gas, the pressure in the solvent phase is calculated from the analytical solution to the diffusion equation for such a diffusion process. Then, the solvent diffusivity in heavy oil is determined by finding the best match of the numerically calculated pressures with the experimentally measured data. More specifically, the equilibrium, quasi-equilibrium and non-equilibrium boundary conditions at the solvent-heavy oil interface are examined. With the measured pressure decay data and the developed diffusion model, the respective molecular diffusion coefficients of carbon dioxide, methane and propane in heavy oil under practical reservoir conditions are determined. It is found that the non-equilibrium boundary condition is the most applicable at the $\mathrm{CO}_{2}$-heavy oil interface. The determined diffusivity for $\mathrm{CH}_{4}$-heavy oil system is insensitive to the interface boundary condition. The mass transfer across $\mathrm{C}_{3} \mathrm{H}_{8}$-heavy oil interface is best described by applying the quasiequilibrium boundary condition. Hence, a proper boundary condition should be used for each solvent-heavy oil system in the determination of diffusion coefficient. Furthermore,
\end{abstract}


in this thesis, a new approach is proposed to determine the so-called equilibrium pressure for each solvent-heavy oil system from its measured solubility versus pressure data. Thus the diffusion coefficient of each solvent in heavy oil can be determined by measuring the pressure decay for a short duration. 


\section{ACKNOWLEDGMENTS}

I wish to acknowledge the following persons or organizations:

- Dr. Yongan (Peter) Gu, my academic advisor, for his excellent guidance, valuable advice and timely encouragement during my Master's degree program. Also, I wish to deliver my sincere appreciation and profound gratitude to $\mathrm{Dr}$. Gu for his supervision, trust and support throughout the course of this research work;

- My past and present research group members, Dr. Daoyong Yang, Mr. Jingquan Li, Mr. Wei Wang, Mr. Peng Luo, and Ms. Hongmei Zhang, for their useful technical discussions during my thesis study. In particular, I am grateful to Mr. Chaodong Yang for his technical assistance and suggestions in this thesis research;

- Petroleum Technology Research Centre (PTRC) for the innovation fund to the research project entitled "Measurement of Solvent Diffusivity in Heavy Oil and Evaluation of Solvent-based Recovery Processes" to Dr. Yongan (Peter) Gu;

- Natural Sciences and Engineering Research Council (NSERC) of Canada for the research grant and the discovery grant to Dr. Yongan (Peter) Gu;

- Faculty of Graduate Studies and Research (FGSR) at the University of Regina for granting the Graduate Research Award in Spring/Summer 2004 and Graduate Scholarship in Fall 2004;

- Mr. Dave Duguid and Mr. Doug Wagner, in Electronic Systems Engineering, University of Regina, for their assistance in resolving an electrical problem in the experiment;

- My classmates on campus, Mr. Naveen Ramachandran and Ms. Jing Zhou, for their occasional help and useful discussions;

- My parents, for their constant support and inspiration throughout my entire education. 


\section{TABLE OF CONTENTS}

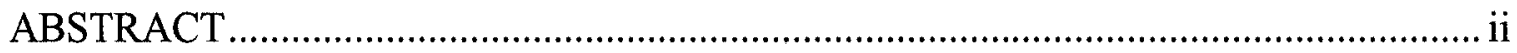

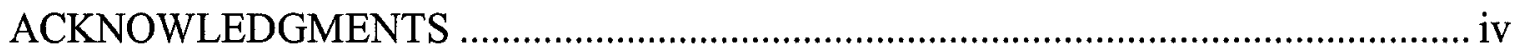

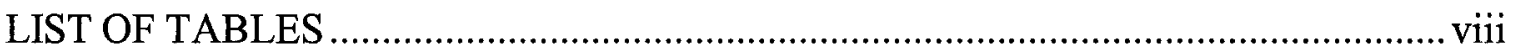

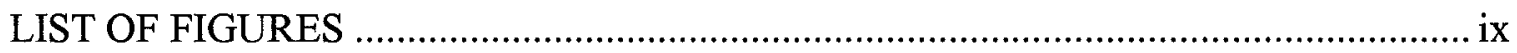

LIST OF APPENDICES ................................................................................ xiii

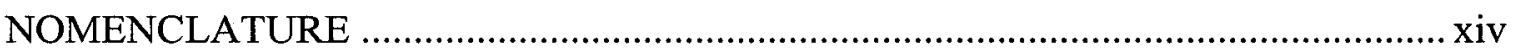

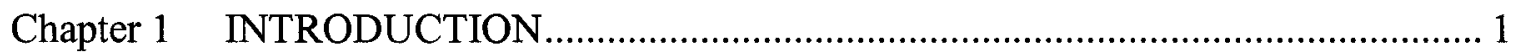

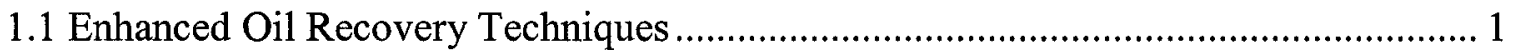

1.2 Importance of Diffusion Coefficient................................................................. 4

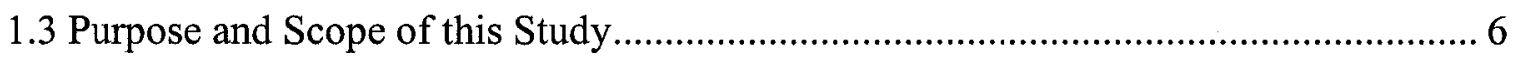

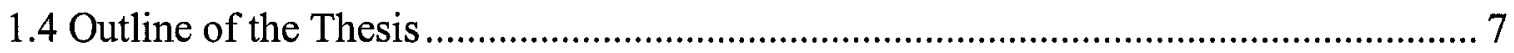

Chapter 2 LITERATURE REVIEW .................................................................... 8

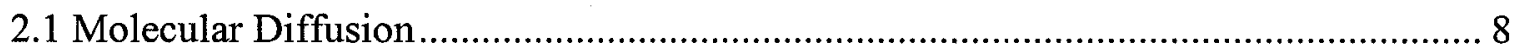

2.2 Theoretical Methods for Predicting Diffusivity................................................. 9

2.3 Experimental Methods for Measuring Diffusivity ........................................... 12

2.3.1 Conventional methods ............................................................................... 13

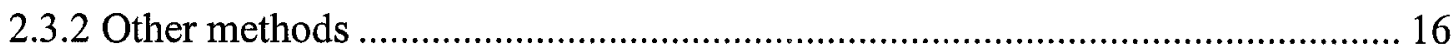

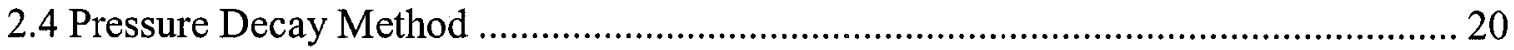

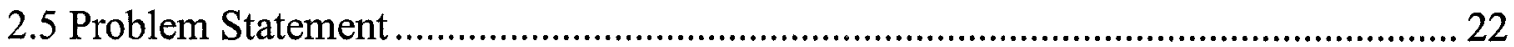

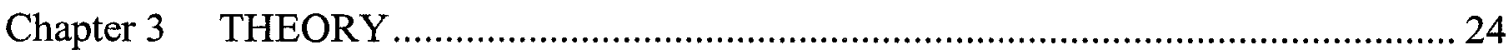

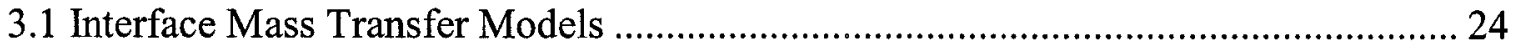

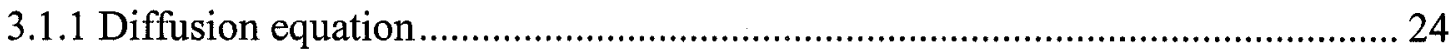

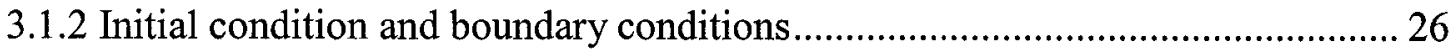


3.1.3 Non-dimensionalization .......................................................................... 28

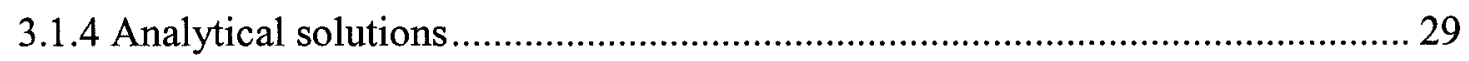

3.2 Calculated Pressure Decay Curves ......................................................................... 33

3.3 Determination of Equilibrium Pressure …………….............................................. 35

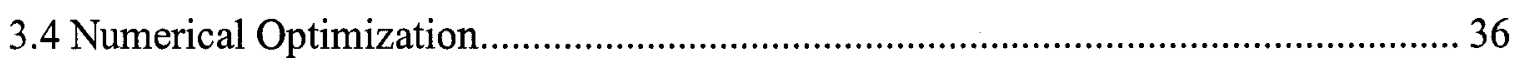

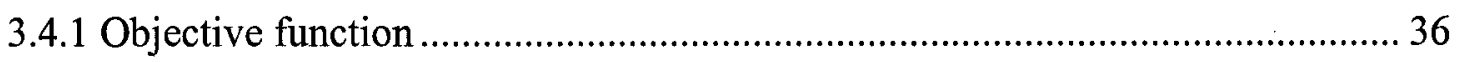

3.4.2 One-parameter simultaneous search method...................................................... 37

3.4.3 Two-parameter simultaneous search method .................................................... 38

Chapter 4 EXPERIMENTAL MEASUREMENTS..................................................... 39

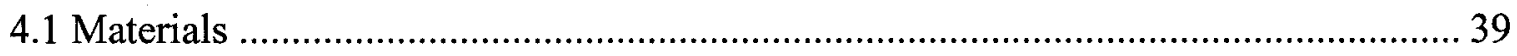

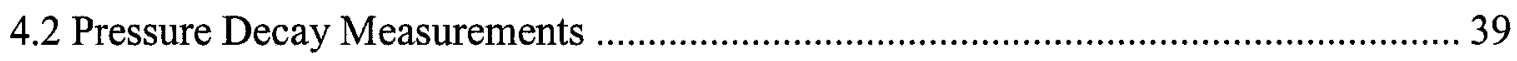

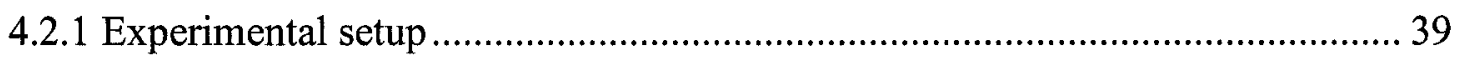

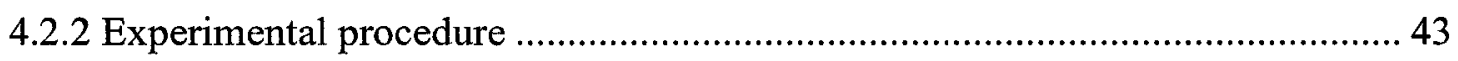

4.2.3 Measured pressure decay data........................................................................... 45

4.3 Solubility Measurements ………………………............................................. 53

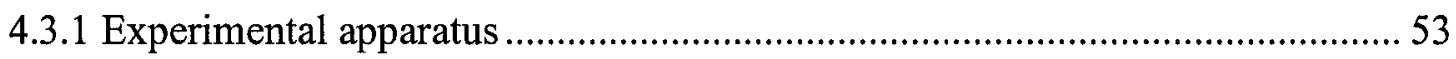

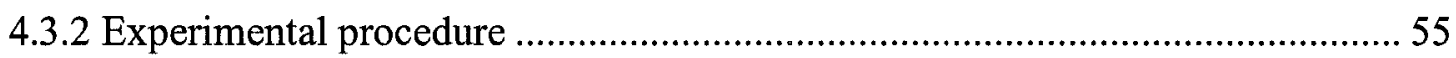

4.3.3 Determination of solubility .......................................................................... 57

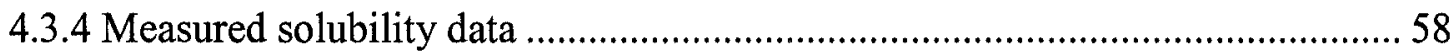

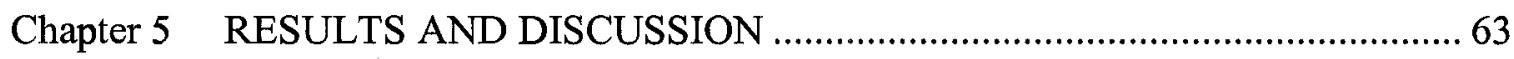

5.1 Interface Mass Transfer Studies Based on the Pressure Decay Data Available in the

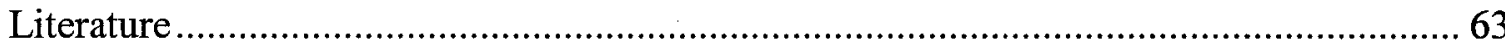

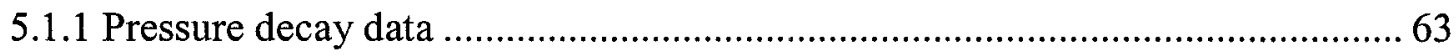

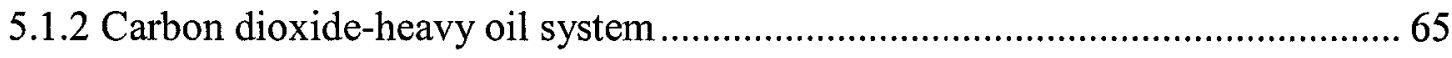

5.1.3 Methane-heavy oil system.............................................................................. 73 
5.2 Determination of Diffusion Coefficients Based on the Measured Pressure Decay Data in this Study

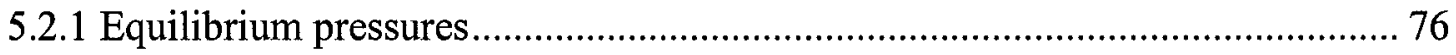

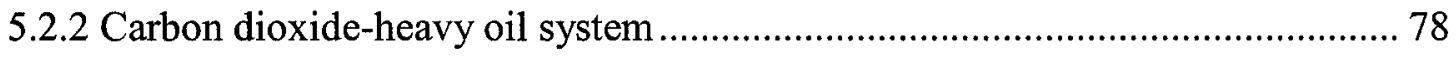

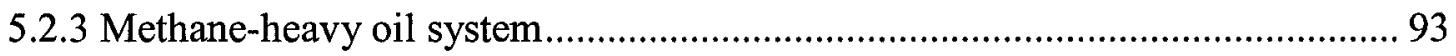

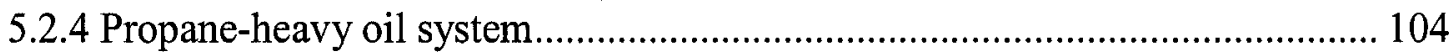

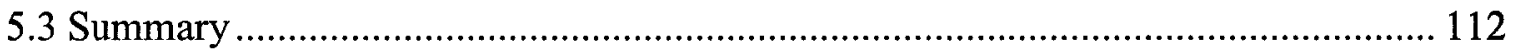

Chapter 6 CONCLUSIONS AND RECOMMENDATIONS.............................. 117

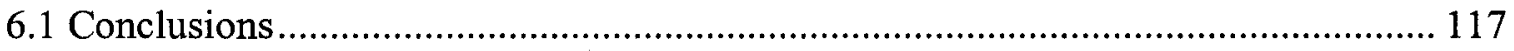

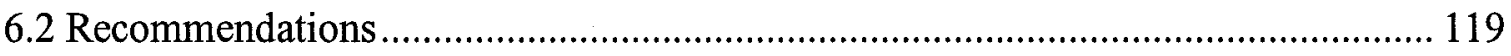

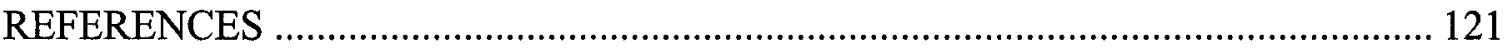




\section{LIST OF TABLES}

Table 2.1 Diffusivities of non-hydrocarbon solvents in crude oil

Table 2.2 Diffusivities of hydrocarbon solvents in crude oil.

Table 4.1 Experimental conditions of pressure decay measurements at $T=23.9^{\circ} \mathrm{C}$

Table 5.1 Experimental conditions of the pressure decay measurements conducted by Zhang et al. (2000) with the heavy oil of viscosity $\mu=5,000 \mathrm{mPa} \cdot \mathrm{s}$ at $T=21^{\circ} \mathrm{C}$ and four parameters required in Eq. [3.12].............64

Table 5.2 Comparison of the results obtained in this study with the previously published data for $\mathrm{CO}_{2}$-heavy oil system (Zhang et al., 2000) at $T=21^{\circ} \mathrm{C}$.

Table 5.3

Comparison of the results obtained in this study with the previously published data for $\mathrm{CH}_{4}$-heavy oil system (Zhang et al., 2000) at $T=21^{\circ} \mathrm{C}$

Table 5.4a Comparison of diffusion coefficients at different diffusion times for $\mathrm{CO}_{2}$-heavy oil system (Run-1) at $T=23.9^{\circ} \mathrm{C}$.

Table 5.4b Comparison of diffusion coefficients at different diffusion times for $\mathrm{CO}_{2}$-heavy oil system (Run-2) at $T=23.9^{\circ} \mathrm{C}$

Table 5.5a

Comparison of diffusion coefficients at different diffusion times for

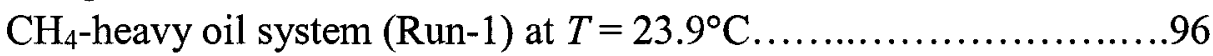

Table 5.5b Comparison of diffusion coefficients at different diffusion times for $\mathrm{CH}_{4}$-heavy oil system (Run-2) at $T=23.9^{\circ} \mathrm{C}$

Table 5.6a Comparison of diffusion coefficients at different diffusion times for

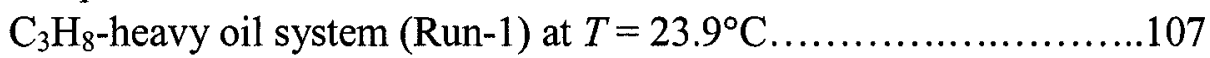

Table 5.6b Comparison of diffusion coefficients at different diffusion times for $\mathrm{C}_{3} \mathrm{H}_{8}$-heavy oil system (Run-2) at $T=23.9^{\circ} \mathrm{C} \ldots \ldots \ldots \ldots \ldots \ldots . \ldots 108$

Table 5.7 Summary of experimental results for three solvent-heavy oil systems at $T=23.9^{\circ} \mathrm{C}$ 


\section{LIST OF FIGURES}

Figure 3.1 Schematic of solvent-heavy oil system inside a closed diffusion cell

Figure 4.1 Block diagram of the experimental setup used to measure the solventheavy oil diffusion coefficient at constant temperature.

Figure 4.2 High-precision absolute pressure transducer used to measure the decaying pressure in the diffusion cell...............................42

Figure 4.3 The experimental setup and the personal computer-based on-line data acquisition system for measuring the solvent-heavy oil diffusion coefficient by using the pressure decay method.....

Figure 4.4a Measured pressure decay data for $\mathrm{CO}_{2}$-heavy oil system (Run-1) at $T=23.9^{\circ} \mathrm{C}$

Figure 4.4b Measured pressure decay data for $\mathrm{CO}_{2}$-heavy oil system (Run-2) at $T=23.9^{\circ} \mathrm{C}$

Figure 4.5a Measured pressure decay data for $\mathrm{CH}_{4}$-heavy oil system (Run-1) at

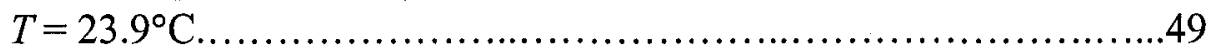

Figure 4.5b Measured pressure decay data for $\mathrm{CH}_{4}$-heavy oil system (Run-2) at $T=23.9^{\circ} \mathrm{C}$

Figure 4.6a Measured pressure decay data for $\mathrm{C}_{3} \mathrm{H}_{8}$-heavy oil system (Run-1) at $T=23.9^{\circ} \mathrm{C}$

Figure 4.6b Measured pressure decay data for $\mathrm{C}_{3} \mathrm{H}_{8}$-heavy oil system (Run-2) at

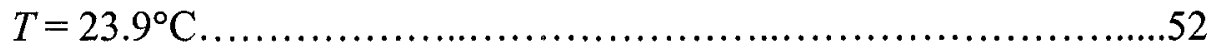

Figure 4.7 Schematic of the vapor-liquid equilibrium (VLE) apparatus used to measure the solubility of a solvent in heavy oil ........................54

Figure 4.8 Vapor-liquid equilibrium (VLE) apparatus used to measure the solubility of a solvent in heavy oil .56

Figure 4.9 Measured solubility of $\mathrm{CO}_{2}$ in heavy oil as a function of pressure at $T=23.9^{\circ} \mathrm{C}$

Figure 4.10 Measured solubility of $\mathrm{CH}_{4}$ in heavy oil as a function of pressure at $T=23.9^{\circ} \mathrm{C}$. 
Figure 4.11 Measured solubility of $\mathrm{C}_{3} \mathrm{H}_{8}$ in heavy oil as a function of pressure at $T=23.9^{\circ} \mathrm{C}$

Figure 5.1 Variations of the average pressure difference $\Delta P_{\text {ave }}$ with the diffusion coefficient $D$ under the equilibrium and quasi-equilibrium boundary conditions for $\mathrm{CO}_{2}$-heavy oil system, based on the pressure decay data published by Zhang et al. (2000).

Figure 5.2 Variations of the average pressure difference $\Delta P_{\text {ave }}$ with the diffusion coefficient $D$ under the non-equilibrium boundary condition for $\mathrm{CO}_{2-}$ heavy oil system, based on the pressure decay data published by Zhang et al. (2000).

Figure 5.3 Variations of the average pressure difference $\Delta P_{\text {ave }}$ with the interface mass transfer coefficient $k$ in the case of $k_{\mathrm{D}}=0$ for $\mathrm{CO}_{2}$-heavy oil system, based on the pressure decay data published by Zhang et al. (2000)

Figure 5.4

History matching of the calculated pressure vs. time curves for the three different BCs (Equilibrium, Quasi-equilibrium and Non-equilibrium) with the experimental data for $\mathrm{CO}_{2}$-heavy oil system (Zhang et al.,

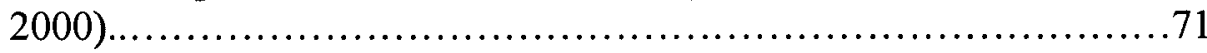

Figure 5.5 Variations of the average pressure difference $\Delta P_{\text {ave }}$ with the diffusion coefficient $D$ under the equilibrium, quasi-equilibrium and nonequilibrium boundary conditions for $\mathrm{CH}_{4}$-heavy oil system based on the pressure decay data published by Zhang et al. (2000)..................75

Figure 5.6 History matching of the calculated pressure vs. time curves for the three different BCs (Equilibrium, Quasi-equilibrium and Non-equilibrium) with the experimental data for $\mathrm{CH}_{4}$-heavy oil system (Zhang et al.

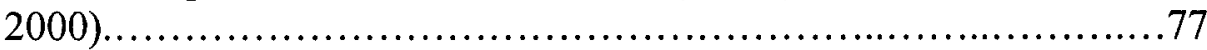

Figure 5.7 The equilibrium pressures determined from the measured solubility data for $\mathrm{CO}_{2}$-heavy oil system at $T=23.9^{\circ} \mathrm{C} \ldots \ldots \ldots \ldots \ldots \ldots \ldots \ldots \ldots$

Figure 5.8 The equilibrium pressures determined from the measured solubility data for $\mathrm{CH}_{4}$-heavy oil system at $T=23.9^{\circ} \mathrm{C} \ldots \ldots \ldots \ldots \ldots \ldots \ldots \ldots \ldots . \ldots \ldots$

Figure 5.9 The equilibrium pressures determined from the measured solubility data

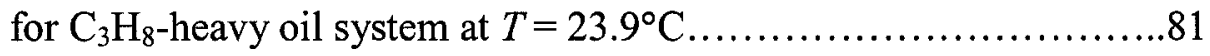

Figure 5.10a Measured pressure decay data and the best-fit curves at different diffusion times for $\mathrm{CO}_{2}$-heavy oil system (Run-1) at $T=23.9^{\circ} \mathrm{C} \ldots \ldots .82$ 
Figure 5.10b Measured pressure decay data and the best-fit curves at different diffusion times for $\mathrm{CO}_{2}$-heavy oil system (Run-2) at $T=23.9^{\circ} \mathrm{C}$

Figure 5.11a History matching of the calculated pressures under three different BCs with the experimental data ( $t=5$ days) for $\mathrm{CO}_{2}$-heavy oil system (Run-

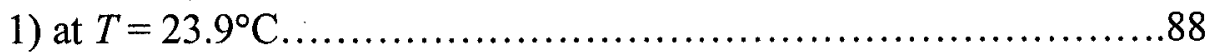

Figure 5.11b History matching of the calculated pressures under three different $\mathrm{BCs}$ with the experimental data ( $t=5$ days) for $\mathrm{CO}_{2}$-heavy oil system (Run-

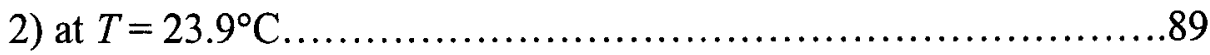

Figure 5.12a Dimensionless solvent concentration profiles for $\mathrm{CO}_{2}$-heavy oil system (Run-1) at different times under the non-equilibrium boundary condition $\left(D=0.94 \times 10^{-9} \mathrm{~m}^{2} / \mathrm{s}\right.$ and $\left.k_{\mathrm{D}}=19.20\right) \ldots \ldots \ldots \ldots \ldots \ldots \ldots \ldots . . . . \ldots 1$

Figure 5.12b Dimensionless solvent concentration profiles for $\mathrm{CO}_{2}$-heavy oil system (Run-2) at different times under the non-equilibrium boundary condition $\left(D=0.92 \times 10^{-9} \mathrm{~m}^{2} / \mathrm{s}\right.$ and $\left.k_{\mathrm{D}}=19.90\right)$.

Figure 5.13a Measured pressure decay data and the best-fit curves at different diffusion times for $\mathrm{CH}_{4}$-heavy oil system (Run-1) at $T=23.9^{\circ} \mathrm{C} \ldots \ldots .94$

Figure 5.13b Measured pressure decay data and the best-fit curves at different diffusion times for $\mathrm{CH}_{4}$-heavy oil system (Run-2) at $T=23.9^{\circ} \mathrm{C} \ldots \ldots .95$

Figure 5.14a History matching of the calculated pressures under three different BCs with the experimental data $\left(t=5\right.$ days) for $\mathrm{CH}_{4}$-heavy oil system (Run1) at $T=23.9^{\circ} \mathrm{C}$

Figure 5.14b

History matching of the calculated pressures under three different BCs with the experimental data ( $t=5$ days) for $\mathrm{CH}_{4}$-heavy oil system (Run2) at $T=23.9^{\circ} \mathrm{C}$

Figure 5.15a Dimensionless solvent concentration profiles for $\mathrm{CH}_{4}$-heavy oil system (Run-1) at different times under the non-equilibrium boundary

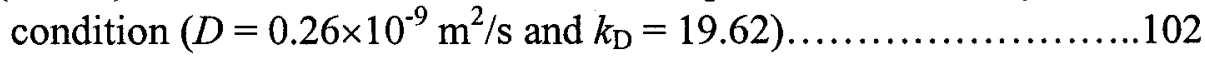

Figure 5.15b Dimensionless solvent concentration profiles for $\mathrm{CH}_{4}$-heavy oil system (Run-2) at different times under the non-equilibrium boundary condition $\left(D=0.23 \times 10^{-9} \mathrm{~m}^{2} / \mathrm{s}\right.$ and $\left.k_{\mathrm{D}}=97.00\right)$.

Figure 5.16a Measured pressure decay data and the best-fit curves at different diffusion times for $\mathrm{C}_{3} \mathrm{H}_{8}$-heavy oil system (Run-1) at $T=23.9^{\circ} \mathrm{C} \ldots . . .105$ 
Figure 5.16b Measured pressure decay data and the best-fit curves at different diffusion times for $\mathrm{C}_{3} \mathrm{H}_{8}$-heavy oil system (Run-2) at $T=23.9^{\circ} \mathrm{C} . \ldots . .106$

Figure 5.17a History matching of the calculated pressures under three different BCs with the experimental data ( $t=5$ days) for $\mathrm{C}_{3} \mathrm{H}_{8}$-heavy oil system (Run1) at $T=23.9^{\circ} \mathrm{C}$

Figure $5.17 \mathrm{~b} \quad$ History matching of the calculated pressures under three different BCs with the experimental data ( $t=5$ days) for $\mathrm{C}_{3} \mathrm{H}_{8}$-heavy oil system (Run2) at $T=23.9^{\circ} \mathrm{C}$

Figure 5.18a Dimensionless solvent concentration profiles for $\mathrm{C}_{3} \mathrm{H}_{8}$-heavy oil system (Run-1) at different times under the quasi-equilibrium boundary condition $\left(D=1.10 \times 10^{-9} \mathrm{~m}^{2} / \mathrm{s}\right)$.

Figure 5.18b Dimensionless solvent concentration profiles for $\mathrm{C}_{3} \mathrm{H}_{8}$-heavy oil system (Run-2) at different times under the quasi-equilibrium boundary condition $\left(D=0.71 \times 10^{-9} \mathrm{~m}^{2} / \mathrm{s}\right)$. 114 


\section{LIST OF APPENDICES}

Appendix A: Analytical Solutions to Diffusion Equation.

Appendix A.1 Derivation of analytical solution given in Eq. [3.10] for the equilibrium

$\mathrm{BC}$

Appendix A.2 Derivation of analytical solution given in Eq. [3.15] for the nonequilibrium $\mathrm{BC}$

Appendix B: Calculation of Compressibility Factor

Appendix B.1 Lee-Kesler correlation 138

Appendix B.2 Hall-Yarborough equation 140

Appendix C: Computer Programs

Appendix C.1 FORTRAN program for the determination of the equilibrium pressure from the measured solubility data

Appendix C.2 FORTRAN program for the diffusivity determination by using the equilibrium $\mathrm{BC}$ at the solvent-heavy oil interface.

Appendix C.3 FORTRAN program for the diffusivity determination by using the quasiequilibrium $\mathrm{BC}$ at the solvent-heavy oil interface.

Appendix C.4 FORTRAN program for the diffusivity determination by using the nonequilibrium $\mathrm{BC}$ at the solvent-heavy oil interface.

Appendix C.5 FORTRAN subroutine program for the calculation of compressibility factor by using the Lee-Kesler correlation

Appendix C.6 FORTRAN subroutine program for the calculation of compressibility factor by using the Hall-Yarborough equation 


\section{NOMENCLATURE}

Notations

$B \quad$ Dimensionless constant defined in Eq. [B.4]

$b_{1}, b_{2}, b_{3}, b_{4}$ Dimensionless constants used in Eq. [B.4]

$C$ Dimensionless molar concentration of solvent in the body of the thesis or dimensionless constant defined in Eq. [B.5] of Appendix B.1

$c \quad$ Molar concentration of diffusing material A (or solvent) in diffusing medium B (or heavy oil), mole $/ \mathrm{m}^{3}$

$c_{1}, c_{2}, c_{3}, c_{4}$ Dimensionless constants used in Eq. [B.5]

$c_{\text {sat }} \quad$ Molar saturation concentration of solvent in heavy oil, mole $/ \mathrm{m}^{3}$

$c_{\mathrm{u}} \quad$ Uniform molar concentration of solvent in heavy oil for $k_{\mathrm{D}}=0, \mathrm{~mole} / \mathrm{m}^{3}$

$D \quad$ Molecular diffusion coefficient of a gas (solvent) in liquid (heavy oil), $\mathrm{m}^{2} / \mathrm{s}$, or dimensionless constant defined in Eq. [B.6]

$D_{\mathrm{AB}} \quad$ Molecular diffusion coefficient of material A in material $\mathrm{B}, \mathrm{m}^{2} / \mathrm{s}$

$d_{1}, d_{2} \quad$ Dimensionless constants used in Eq. [B.6]

$E_{D A B} \quad$ Activation energy for diffusion of material A in material B, $\mathrm{J}$

$E_{\mu B} \quad$ Activation energy for viscosity of material B, $\mathrm{J}$

$j \quad$ Mass transfer flux, mole $/ \mathrm{m}^{2} / \mathrm{s}$

k Boltzmann constant $\left(\mathbf{k}=1.3807 \times 10^{-23} \mathrm{~J} / \mathrm{K}\right)$

$k$ Interface mass transfer coefficient, $\mathrm{m} / \mathrm{s}$, or dummy variable used in Appendix A.1 and Appendix A.2

$k_{\mathrm{D}} \quad$ Mass-transfer Biot number

$k_{1}, k_{2} \quad$ Parameters used in Eq. [3.12], day

$L \quad$ Height of heavy oil in the diffusion cell, $\mathrm{m}$

$M \quad$ Molecular weight, $\mathrm{kg} / \mathrm{kmole}$, or dummy variable used in Appendix A.1 and Appendix A.2

$M^{\prime} \quad$ Dummy variable used in Appendix A.1 and Appendix A.2

$m \quad$ Number of time series, $t=t_{i}, i=1,2,3, \ldots, m$

$m_{1}, m_{2} \quad$ Parameters used in Eq. [3.12], $\mathrm{kPa}$

$m_{\mathrm{oil}} \quad$ Mass of heavy oil in the diffusion cell, $\mathrm{kg}$ 
$N \quad$ Avagadro's number $\left(N=6.023 \times 10^{23}\right.$ molecules/mole $)$, or dummy variable used in Appendix A.1 and Appendix A.2

$N^{\prime} \quad$ Dummy variable used in Appendix A.1 and Appendix A.2

$N_{\text {eq }} \quad$ Number of moles of solvent remaining in solvent phase at the equilibrium pressure, mole

$N_{\mathrm{g}} \quad$ Number of moles of solvent in the gaseous solvent phase, mole

$N_{\mathrm{i}} \quad$ Initial total number of moles of solvent, mole

$N_{0} \quad$ Total number of moles of solvent in the heavy oil phase, mole

$n \quad$ Integer used in Eqs. [3.10], [3.14] and [3.15]

$P \quad$ Pressure, $\mathrm{kPa}$

$P_{\mathrm{c}} \quad$ Critical pressure, $\mathrm{kPa}$

$P_{\text {cal }} \quad$ Calculated pressure, $\mathrm{kPa}$

$P_{\text {eq }} \quad$ Equilibrium pressure, $\mathrm{kPa}$

$P_{\exp } \quad$ Measured pressure, $\mathrm{kPa}$

$P_{\mathrm{f}} \quad$ Final pressure, $\mathrm{kPa}$

$P_{\mathrm{i}} \quad$ Initial pressure, $\mathrm{kPa}$

$P_{\mathrm{pc}} \quad$ Pseudo critical pressure, $\mathrm{kPa}$

$P_{\mathrm{r}} \quad$ Reduced pressure

$p_{\mathrm{pr}} \quad$ Pseudo reduced pressure

$p, q \quad$ Dummy variables used in the derivation of Eqs. [3.10] and [3.15]

$R \quad$ Universal gas constant $(R=8.314 \mathrm{~kJ} / \mathrm{kmole} / \mathrm{K})$, or dummy variable used in Appendix A.1 and Appendix A.2

$\bar{R} \quad$ Radius of gyration, $\stackrel{\circ}{\mathbf{A}}$

$r_{A} \quad$ Radius of the diffusing molecule of material A, m

$t \quad$ Time, $\mathrm{s}$

$\bar{t} \quad$ Reciprocal of pseudo reduced temperature

$T \quad$ Temperature, $\mathrm{K}$

$T_{\mathrm{c}} \quad$ Critical temperature, $\mathrm{K}$

$T_{\mathrm{pc}} \quad$ Pseudo critical temperature, $\mathrm{K}$

$T_{\mathrm{r}} \quad$ Reduced temperature

$V \quad$ Volume, $\mathrm{m}^{3}$ 


$\begin{array}{ll}v_{\mathrm{A}}, v_{\mathrm{B}} & \text { Molar volume of material A and material B, } \mathrm{m}^{3} / \mathrm{mole} \\ v_{\mathrm{CA}}, v_{\mathrm{CB}} & \text { Critical molar volume of material A and material B, } \mathrm{m}^{3} / \mathrm{mole} \\ V_{\mathrm{i}} & \text { Initial volume of heavy oil in pressure cell, } \mathrm{m}^{3} \\ V_{\mathrm{f}} & \text { Final volume of heavy oil in pressure cell, } \mathrm{m}^{3} \\ V_{\text {solvent }} & \text { Volume of solvent, } \mathrm{m}^{3} \\ V_{\mathrm{r}} & \text { Reduced volume } \\ v^{*} & \text { Volumetric average velocity, } \mathrm{m} / \mathrm{s} \\ X & \text { Dimensionless distance } \\ x & \text { Normal distance in the direction of diffusion, or distance from the bottom of } \\ y & \text { diffusion cell as shown in Fig. } 3.1, \mathrm{~m} \\ \mathrm{Z} & \text { "Reduced" density defined in Eq. [B.8] }\end{array}$

Greek letters

$\alpha, \beta \quad$ Correlation constants defined in Eq. [2.5], or dimensionless pressures defined in Eq. [3.13]

$\beta, \gamma \quad$ Dimensionless constants used in Eq. [B.2]

$\Delta P_{\text {ave }} \quad$ Average pressure difference, $\mathrm{kPa}$

$\Delta P_{i} \quad$ Pressure difference at $t=t_{i}, i=1,2,3, \ldots, m, \mathrm{kPa}$

$\Delta X \quad$ Integral interval of the dimensionless distance in numerical integration

$\kappa_{1}, \kappa_{2} \quad$ Dimensionless times defined in Eq. [3.13]

$\lambda_{n} \quad$ Eigen values or positive roots of the characteristic equation, $\tan \lambda_{n}=k_{D} / \lambda_{n}$

$\mu \quad$ Viscosity, $\mathrm{mPa} \cdot \mathrm{s}$

$\xi_{\mathrm{A}} \quad$ Number of molecules around a central molecule of material A on the same plane

$\tau \quad$ Dimensionless time or mass-transfer Fourier number

$v \quad$ Dummy variable used in Appendix A.1

$\chi \quad$ Solubility of solvent in heavy oil, $\mathrm{g}$ solvent $/ 100 \mathrm{~g}$ heavy oil

$\omega \quad$ Dummy variable used Appendix A.1 and Appendix A.2, or acentric factor 


\section{Superscripts}
(o) Simple fluid
(r) Reference fluid

\section{Subscripts}

0 Intrinsic value

A Material A

ave Average value

B Material B

c Critical value

CA Critical value of material A

CB Critical value of material B

cal Calculated value

eq Value at the equilibrium condition

exp Measured data

f Value at the final condition

g Value in the solvent (gas) phase

i Value at the initial condition, or at $t=t_{i}, i=1,2,3, \ldots, m$

$n \quad \mathrm{n}^{\text {th }}$ eigen value defined in Eq. [3.15], and the positive roots of the characteristic equation $\tan \lambda_{n}=k_{D} / \lambda_{n}$

- Value in the oil (liquid) phase

oil Heavy oil

pc Pseudo critical value

pr Pseudo reduced value

$\mathrm{r} \quad$ Reduced value

sat Value at the saturation condition

solvent Solvent

$\mathrm{u} \quad$ Uniform value for $k_{\mathrm{D}}=0$ 


\section{Chapter 1 INTRODUCTION}

\subsection{Enhanced Oil Recovery Techniques}

With the depletion of conventional crude oil reserves, heavy oil and bitumen deposits in Canada have a great potential to meet the future demand for petroleum products. It has been estimated that these deposits constitute about 1.7 trillion barrels (Young, 2003). They naturally exist in the earth crust and are recognized as one of the most complex mixtures of organic compounds. In general, crude oil with viscosity in the range of 10 to $10,000 \mathrm{mPa} \cdot \mathrm{s}$ is considered as heavy oil, while that with viscosity greater than $10,000 \mathrm{mPa} \cdot \mathrm{s}$ under the reservoir pressure and temperature conditions is termed as extra heavy crude oil or bitumen (Strausz, 1989; Miller, 1994). After both primary and secondary oil recovery in Canadian heavy oil/bitumen reservoirs, approximately $70-80 \%$ of the original oil in place (OOIP) remains unrecoverable below the economic limit (Miller et al., 2002). This is attributed to several factors, such as extremely high viscosity of heavy crude oil, large interfacial tension between the injected fluid and heavy oil, high capillary pressure, and unfavorable reservoir lithology. Numerous studies have been carried out to investigate these factors and various tertiary recovery or enhanced oil recovery (EOR) techniques have been proposed to recover the remaining heavy oil from the hydrocarbon reservoirs (Green and Willhite, 1998).

In a broad classification, the EOR techniques include chemical methods, thermal methods and solvent-based methods. The basic chemical EOR processes are alkaline, surfactant and polymer flooding. Both the alkaline and surfactant flooding processes are based on the similar mechanisms, such as the interfacial tension reduction between the 
injected fluid and the reservoir fluids to low or ultra-low values (Wang and $\mathrm{Gu}, 2003$ ) and wettability alteration of reservoir rock. Polymer flooding is a chemically augmented or improved water flood process, which enhances the oil recovery by increasing both the areal and vertical sweep efficiencies. This is facilitated by adding polymer in small concentrations into the injected fluid to reduce its mobility. Therefore, polymer flood process helps to prevent or eliminate the early breakthrough of the injected fluid, i.e., channeling. Generally speaking, these chemical EOR processes are technically complex and can be successful only in light and medium crude oil reservoirs.

To recover highly viscous heavy oil/bitumen, thermal-based recovery methods are often used. In these methods, the viscosity of crude oil is reduced by heating the reservoir. Currently applied thermal methods include steam flooding, cyclic steam stimulation (CSS), in situ combustion (ISC) and steam assisted gravity drainage (SAGD). The maximum recovery in the CSS process seldom exceeds $20 \%$, which is usually followed by steam flooding process. For ISC to be successful, the original oil should have sufficiently low viscosity $(<1,000 \mathrm{mPa} \cdot \mathrm{s})$ so that it can be displaced away from the combustion zone into the production well (Moore et al., 1994). Although SAGD process holds a great potential to recover heavy oil reserves, it is often uneconomical if the pay zone is not thick enough or there is an active bottom water aquifer (Yazdani and Maini, 2004). Apart from the energy inefficiency, other major concerns, such as the presence of overlying gas cap, low thermal conductivity, treatment/disposal of effluent water, huge source for the supply of fresh water and possibility of formation damage by clay swelling, also limit the thermal methods. In general, the thermal methods cause excessive heat loss and require huge amounts of steam and extensive surface facilities (Miller, 1987). 
On the other hand, solvent-based methods utilize gaseous solvents that dissolve into the heavy oil to reduce its viscosity (Butler and Yee, 2002). Presence of dissolved solvent gas in heavy oil may further enhance oil recovery by generating a saturated displacement front that is caused due to oil swelling in reservoir pores (Grogan and Pinczewski, 1987) and by reducing the interfacial tension between the heavy oil and the reservoir brine (Yang and $\mathrm{Gu}, 2004$ ). Since a gaseous solvent injection process does not involve the heat loss that is a major technical problem associated with the thermal recovery methods, it is relatively economical and thus it is currently under active investigation at both laboratory and field scales.

At present, vapor extraction (VAPEX) process (Butler and Mokrys, 1991), which is a typical solvent-based oil recovery method, receives special attention for enhancing the heavy oil/bitumen recovery. VAPEX process (Das, 1998) is a modification of the SAGD process. In principle, instead of using steam, a gaseous hydrocarbon solvent is used, whose thermodynamic state is close to its dew point at the reservoir pressure and temperature. Hydrocarbon solvents that are studied include ethane, propane and butane (Das and Butler, 1996). In the VAPEX process, the heavy oil/bitumen is mobilized by the dissolution of hydrocarbon solvent into heavy oil. This process is conducted in a pair of horizontal wells, with an injection well being located above the production well. Vaporized solvents that are injected through the horizontal injection well gradually dissolve into the viscous heavy oil and drastically reduce its viscosity. Consequently, the solvent-enriched oil phase becomes mobile enough to drain down under the influence of gravity into the horizontal production well, which is located near the bottom of the pay zone. 
Another type of solvent-based oil recovery processes is the direct injection of light gases/solvents. Two distinct displacement cases, namely miscible and immiscible flooding, can occur when solvent is injected into a heavy oil reservoir. In practice, the solvents can be either non-hydrocarbon gases, such as carbon dioxide, flue gas, carbon monoxide, air and nitrogen, or hydrocarbon gases, such as natural gas, methane, ethane, propane, butane, liquefied natural gas (LNG) and condensed liquefied petroleum gas (LPG) (Lake, 1989). In consideration of the wide variety of solvents and the types of solvent-based processes and hydrocarbon reservoirs, the major solvents used are carbon dioxide, air, flue gas, methane, propane and butane (Das and Butler, 1998). In the socalled miscible flooding, the injected solvent mixes in any proportion with the heavy oil to be displaced. It should be noted that, in most cases, the miscibility is achieved by multiple contacts between the injected solvent and heavy oil at pressures above the minimum miscibility pressure (MMP) (Srivastava et al., 1994; Jaubert et al., 2002). If the injected solvent is miscible with the heavy oil, the interfacial tension at the displacing front becomes zero and the capillary force that traps the heavy oil in the reservoir formation or matrix disappears. Thus, this results in efficient displacement of heavy oil. During the immiscible displacement, the solvent flooding can be either water alternating gas (WAG) injection or water injection with surfactant to generate foams. In general, the purpose of the solvent injection in the immiscible displacement is to improve the efficiency of the process by reducing the capillary force.

\subsection{Importance of Diffusion Coefficient}

The efficiency of a solvent-based oil recovery process is largely dependent on the dissolution of solvent in heavy oil and the resultant changes of heavy oil properties. 
Recent studies have shown that the field performance of the solvent-based processes strongly depends on the mass transfer of solvents in heavy oil under reservoir conditions (Boustani and Maini, 2001). Several mass transfer models have also been studied to evaluate the recovery rates during the solvent-based processes (Cuthiell et al., 2003). It has been identified that the molecular diffusion (Grogan and Pinczewski, 1987) and convective dispersion (Yazdani and Maini, 2004) are the two mass transfer processes in the solvent-based heavy oil recovery. Specifically, the molecular diffusion plays an important role in the mass transfer during the so-called soaking time, whereas the convective dispersion is dominant at the time of solvent injection. The molecular diffusion is considered to be important in solvent flooding since it allows gaseous solvents to dissolve in the heavy oil and then inhibits viscous fingering, delays gas breakthrough and improves the oil recovery. During the VAPEX process, the molecular diffusion controls the rate at which the solvent vapor dissolves in the heavy oil, since the magnitude of molecular diffusion coefficient in the high viscosity liquid is very small, in the order of $0.1 \times 10^{-9} \mathrm{~m}^{2} / \mathrm{s}$ (Hiss and Cussler, 1973). Therefore, both the field design and reservoir simulation of a solvent-injection operation need to determine the amount of solvent required for injection and the time taken to achieve the desired mobility of reservoir fluids. This requires the solubility and diffusivity of a solvent in heavy oil under reservoir conditions. Although numerous solubility data are available in the literature, diffusivity data for the solvent-heavy oil systems of interest are scarce. Thus, to better understand the mass transfer by the molecular diffusion, it is desirable to determine the diffusivity data of solvents in heavy oil under practical reservoir conditions. 


\subsection{Purpose and Scope of this Study}

The purpose of this study is to measure the diffusion coefficients of solvents in heavy oil by using the so-called pressure decay method. This non-intrusive experimental method is based on the fact that the pressure in the solvent phase decays as the molecular diffusion of solvent into heavy oil proceeds in a closed diffusion cell. Such a diffusion process continues until the heavy oil is completely saturated with the solvent. In the experiment, the decaying pressure in the solvent phase is accurately measured at constant temperature. With the measured pressure versus time data and a theoretical model developed for such a diffusion process, the respective molecular diffusion coefficients of carbon dioxide, methane and propane in heavy oil are determined at constant reservoir temperature. Specifically, in the theoretical model, the conservation of mass and the equation of state for a real gas are used to predict the pressure in the solvent phase from the analytical solution to the diffusion equation. Therefore, the solvent diffusivity in heavy oil is determined by finding the best match of the numerically predicted pressures with the experimentally measured data.

In this thesis study, technical efforts are made to accurately measure the pressure decay data for all the three solvent-heavy oil systems. Moreover, in the development of theoretical model, the respective analytical solution to the diffusion equation is obtained for each boundary condition at the solvent-heavy oil interface. More specifically, the equilibrium, quasi-equilibrium and non-equilibrium boundary conditions are applied to the above-mentioned three solvent-heavy oil systems, respectively. These three boundary conditions are analysed, based on the experimental data available in the literature and the accurately measured pressure decay data in this study. The three different boundary 
conditions are examined by using the developed diffusion model. Thus, a better understanding is achieved regarding the effects of these three rather different boundary conditions on the determined diffusion coefficient. As the diffusion process takes a long time to complete, a new strategy is adopted to determine the diffusion coefficient of a solvent in heavy oil based on the pressure decay data measured in a short duration, if the measured solubility data of the same solvent-heavy oil system is known. In this thesis study, the solubility data for carbon dioxide, methane and propane in the given heavy oil are also measured for a certain pressure range at constant temperature.

\subsection{Outline of the Thesis}

The thesis is composed of six chapters. Specifically, Chapter 1 gives an introduction to the thesis research topic together with the purpose and scope of this study for the determination of diffusion coefficient of a gas solvent in heavy oil. Chapter 2 reviews the various theoretical and experimental methods available for determining the diffusion coefficient of a gas in a liquid. This chapter also includes the problem statement of the present research topic. Chapter 3 presents three interface mass transfer models developed in this study and the numerical procedure for determining the diffusion coefficient. Chapter 4 describes the experimental setup and procedure for conducting the pressure decay measurement and the solubility measurement of a solvent in a heavy oil sample. This chapter also provides detailed experimental results for carbon dioxide, methane and propane. Chapter 5 discusses the diffusivity data obtained by using the three interface mass transfer models developed in Chapter 3 and the experimental results presented in Chapter 4. Finally, Chapter 6 contains a summary of major conclusions of this thesis study and some recommendations for future research. 


\section{Chapter 2 LITERATURE REVIEW}

\subsection{Molecular Diffusion}

Molecular diffusion is the random movement of molecules of a specific species from a high concentration region to a low concentration region. The physics of the molecular diffusion process has been extensively reviewed by Jost (1960), Hirschfelder et al. (1967), Cussler (1984) and Bird et al. (2002). In general, the Fick's first law is used to describe the molecular diffusion process in one-dimensional case:

$$
j=-D \frac{\partial c}{\partial x}
$$

where $j$ is the mass transfer rate by molecular diffusion per unit surface area or the mass transfer flux of the diffusing substance; $c$ is the concentration of the diffusing substance; $x$ is the space coordinate normal to the surface across which the mass transfer occurs; and $D$ is called the diffusion coefficient or simply called diffusivity. Physically, this law states that the mass transfer flux of a species, relative to the medium of propagation, is proportional to its spatial concentration gradient along the direction of mass transfer (Jacobs, 1967). The negative sign in Eq. [2.1] arises because the diffusion occurs in the direction opposite to that of increasing concentration.

In many laboratory experiments for the study of diffusion in non-reacting gas mixtures, certain conditions have to be satisfied in order to measure diffusivity (Ghez, 2001). For example, the system is maintained at constant temperature. There are no external forces acting on the molecules and also the diffusion takes place in one direction only. Under such conditions, Equation [2.1] may be combined with the equation of continuity for the gas species to obtain a second-order partial differential equation: 


$$
\frac{\partial c}{\partial t}=\frac{\partial}{\partial x}\left(D \frac{\partial c}{\partial x}\right)-v^{*} \frac{\partial c}{\partial x}
$$

This is called the Fick's second law of diffusion. For a system within a stationary closed vessel, the volumetric average velocity $v^{*}$ is zero and therefore the second term on the right-hand side of Eq. [2.2] disappears. Also, in most cases for dilute solutions, the diffusivity can be considered as constant, independent of concentration during the diffusion process. Thus, Eq. [2.2] can be simplified as:

$$
\frac{\partial c}{\partial t}=D \frac{\partial^{2} c}{\partial x^{2}}
$$

Mathematically, this equation is usually categorized as the mass-transfer diffusion equation.

\subsection{Theoretical Methods for Predicting Diffusivity}

Extensive reviews on the theoretical methods for predicting diffusivity have been conducted by Hirchfelder et al. (1967) and Reid et al. (1987). Essentially, in the literature, four general theoretical approaches or theories of diffusion have been proposed, which are the hydrodynamic theory, the kinetic theory, the statisticalmechanical theory and the irreversible thermodynamic theory. The hydrodynamic theory is the most widely used approach in terms of practical application. The predictive equations and semi-empirical correlations in calculating the diffusion coefficients are summarized by Mehrotra et al. (1987). Among all the existing empirical equations, some of the most frequently used equations in estimating the diffusivity are selected and described in this section. 
The predictive equations, such as Stokes-Einstein equation and its modified form i.e., Wilke-Chang equation (Wilke and Chang, 1955), are based on the hydrodynamic approach and found to be of limited success in predicting gas-liquid diffusivity at the atmospheric condition for binary and ternary systems. These two equations are generally applicable for predicting diffusivities in dilute binary liquid-liquid systems. The StokesEinstein's equation is given by:

$$
D_{A B}=\frac{\mathbf{k} T}{6 \pi r_{A} \mu_{B}},
$$

where $D_{A B}$ is the diffusivity of gas $\mathrm{A}$ in liquid $\mathrm{B} ; \mathbf{k}$ is the Boltzmann constant; $T$ is the absolute temperature; $\mu_{B}$ is the viscosity of liquid $\mathrm{B}$; and $r_{A}$ is the radius of diffusing molecule of gas A. This equation indicates that the diffusion coefficient changes with the size of the diffusing molecule. It also suggests that the shape of the diffusing species may be an important factor because the Stokes-Einstein equation is only valid for small hard spherical molecules.

Based on the hypothesis put forward by Hayduk and Cheng (1971), the diffusivity of each gas dissolved in a liquid is a unique function of liquid viscosity, provided that the state of molecular aggregation of both the solute and solvent remains essentially unaltered after mixing. The following relation between diffusivity $D$ and viscosity $\mu$ is proposed:

$$
D=\alpha \mu^{-\beta},
$$

where $\alpha$ and $\beta$ are constants for each diffusing substance. On the basis of limited experimental data, it has been concluded that this relation is independent of temperature and solvent composition. The dependence of diffusivity on temperature and composition 
can be accounted through viscosity. However, when the viscosity is increased, the predicted diffusivity becomes much smaller than the measured one.

Akgerman and Gainer (1972) used absolute rate theory with a liquid structure model and proposed an equation to determine the gas-liquid diffusion coefficient, $D_{A B}$, in the form:

$$
D_{A B}=\frac{\mathbf{k} T}{\xi_{A} \mu_{B}}\left(\frac{N}{v_{B}}\right)^{1 / 3}\left(\frac{M_{B}}{M_{A}}\right)^{1 / 2} \exp \left(\frac{E_{\mu B}-E_{D A B}}{R T}\right)
$$

where $\xi_{A}=6\left(\frac{v_{A}}{v_{B}}\right)^{1 / 6}$ represents the number of molecules of material B around a central molecule of material A on the same plane; $N$ is the Avagadro's number; $v_{A}$ and $v_{B}$ are the molar volumes of material $\mathrm{A}$ and $\mathrm{B} ; \mu_{B}$ is the viscosity of liquid $\mathrm{B} ; M_{A}$ and $M_{B}$ denote the respective molecular weights of $\mathrm{A}$ (gas) and $\mathrm{B}$ (liquid); $E_{\mu B}$ represents the activation energy for viscosity of material $\mathrm{B} ; E_{D A B}$ represents the activation energy for diffusion of material $\mathrm{A}$ in material $\mathrm{B}$; and $R$ is the universal gas constant. These two activation energies are calculated by using the respective equations provided in the literature.

Sridhar and Potter (1977) proposed another equation to predict both the gas-liquid and liquid-liquid diffusivity, $D_{A B}$,

$$
D_{A B}=0.088 \frac{v_{C B}^{4 / 3}}{N^{2 / 3}} \frac{R T}{\mu_{B} v_{0}} \frac{1}{v_{C A}^{2 / 3}}
$$

where $v_{C A}$ and $v_{C B}$ are the critical molar volumes of gas $\mathrm{A}$ and liquid $\mathrm{B}$; and $v_{0}=0.31 v_{C B}$ is the intrinsic molar volume. This equation is developed on the basis of the hydrodynamic theory and shows an inverse relationship between the diffusivity and the size of the solute molecule described in terms of the critical molar volume of the solute. 
Umesi and Danner (1981) have shown that the radius of gyration can be effectively employed to represent the size-shape effects on the predicted gas-liquid diffusion coefficient. In this case, the molecular geometry of the species is considered. The formulated correlation for calculating the diffusion coefficient, $D_{A B}$, is given by:

$$
D_{A B}=2.75 \times 10^{-8} \frac{T}{\mu_{B}}\left[\bar{R}_{B} / \bar{R}_{A}\right],
$$

where $\bar{R}_{A}$ and $\bar{R}_{B}$ are the respective radius of gyration of a molecule of material A and a

molecule of material $\mathrm{B} ; \mu_{B}$ is the viscosity of material B. A table of $\bar{R}$ values for 250 compounds is provided by Reid et al. (1987). It should be noted that this equation is applicable only for pure gas diffusing in a pure liquid. However, this equation cannot be applied for heavy oil, as it is a complex mixture of various hydrocarbons.

All the above-mentioned equations are particularly applicable for binary systems at the atmospheric pressure. As the diffusion process of a solvent gas in heavy oil usually takes place under high pressures, these equations are not suitable for accurately determining the diffusivity. Also, the heavy oil is a mixture of complex hydrocarbon components. Therefore, it is essential to determine the diffusion coefficient of solvent in heavy oil under reservoir conditions by using the reliable experimental methods rather than using the existing empirical correlations.

\subsection{Experimental Methods for Measuring Diffusivity}

Experimental methods for measuring the gas diffusivity in liquids can be broadly categorized into conventional and other methods. In the conventional methods, samples of the liquid that contain dissolved gas are taken at various times and analyzed by gas 
chromatography (Sigmund, 1976) or by NMR (Wen et al., 2003) to find the composition of gas. Thus they are the so-called intrusive methods (Upreti, 2000). The extraction of samples in these methods disturbs the system and the composition estimation becomes quite susceptible to experimental errors (Riazi and Whitson, 1993). In addition, the measurement of diffusion coefficient requires compositional analysis, which is expensive and time-consuming. On the other hand, the other methods measure any property change due to diffusion and correlate that with the composition. Such a property change can be solute volatilization rate (Fu and Phillips, 1979), solvent volume (Denoyelle and Bardon, 1984; Renner, 1988), refractive index (Yu, 1984), solvent-heavy oil interface position (Grogan et al., 1988), refraction or absorption of electro-magnetic radiation (Oballa and Butler 1989), system pressure (Riazi, 1996), shape and volume of a pendant oil drop (Yang and $\mathrm{Gu}, 2003$ ). These non-intrusive methods do not disturb the system and several simplifications are made in estimating the diffusion coefficient. Table 2.1 shows the diffusivities of non-hydrocarbon solvent-heavy oil/bitumen systems, which are found from the literature. Similarly, Table 2.2 shows the published diffusivity data for various hydrocarbon solvent-heavy oil/bitumen systems. These two tables include the diffusivities determined by using both intrusive and non-intrusive methods.

\subsubsection{Conventional methods}

Schmidt et al. (1982) reported the diffusivities of carbon dioxide, methane and ethane in Athabasca bitumen at different temperatures and pressures in the range of 20$200^{\circ} \mathrm{C}$ at 4.93 $\mathrm{MPa}$ (See Tables 2.1 and 2.2). A partition cell was used to conduct the diffusion experiment for eight days. After the experiment, the dissolved gas and bitumen 
Table 2.1 Diffusivities of non-hydrocarbon solvents in crude oil.

\begin{tabular}{|c|c|c|c|c|c|}
\hline Solvent & Crude oil & $\begin{array}{c}\text { Pressure } \\
{[\mathrm{MPa}]}\end{array}$ & $\begin{array}{c}\text { Temperature } \\
{\left[{ }^{\circ} \mathrm{C}\right]}\end{array}$ & $\begin{array}{l}\text { Diffusivity } \\
{\left[10^{-9} \mathrm{~m}^{2} / \mathrm{s}\right]}\end{array}$ & Reference \\
\hline \multirow{22}{*}{$\mathrm{CO}_{2}$} & \multirow{3}{*}{$\begin{array}{l}\text { Stock tank } \\
\text { oil }\end{array}$} & \multirow{3}{*}{15} & 66 & 3.0 & \multirow{3}{*}{$\begin{array}{l}\text { Denoyelle and } \\
\text { Bardon (1984) }\end{array}$} \\
\hline & & & 75 & $8.5-9.2$ & \\
\hline & & & 80 & 4.6 & \\
\hline & $\begin{array}{l}\text { Recombined } \\
\text { reservoir oil }\end{array}$ & 13.1 & 54 & 5 & $\begin{array}{c}\text { Grogan and } \\
\text { Pinczewski (1987) }\end{array}$ \\
\hline & $\begin{array}{l}\text { Maljamar } \\
\text { crude oil }\end{array}$ & 5.2 & 25 & 2 & Grogan et al. (1988) \\
\hline & \multirow{8}{*}{$\begin{array}{l}\text { Athabasca } \\
\text { bitumen }\end{array}$} & \multirow{8}{*}{4.93} & 20 & 0.28 & \multirow{8}{*}{ Schmidt (1989) } \\
\hline & & & 50 & 0.50 & \\
\hline & & & 75 & 0.71 & \\
\hline & & & 100 & 0.92 & \\
\hline & & & 125 & 1.15 & \\
\hline & & & 150 & 1.41 & \\
\hline & & & 175 & 1.55 & \\
\hline & & & 200 & 1.75 & \\
\hline & Aberfeldy oil & 1 & 23 & 6 & $\begin{array}{l}\text { Nguyen and Farouq } \\
\text { Ali (1998) }\end{array}$ \\
\hline & $\begin{array}{l}\text { Hamaca } \\
\text { heavy oil }\end{array}$ & 2.84 & 21 & 4.8 & Zhang et al. (2000) \\
\hline & \multirow{7}{*}{$\begin{array}{l}\text { Suncor coker } \\
\text { feed bitumen }\end{array}$} & \multirow{4}{*}{4} & 25 & 0.1335 & \multirow{7}{*}{$\begin{array}{l}\text { Upreti and Mehrotra } \\
\qquad(2002)\end{array}$} \\
\hline & & & 50 & 0.2338 & \\
\hline & & & 75 & 0.3739 & \\
\hline & & & 90 & 0.4280 & \\
\hline & & \multirow{3}{*}{8} & 50 & 0.3980 & \\
\hline & & & 75 & 0.7436 & \\
\hline & & & 90 & 0.9319 & \\
\hline \multirow{8}{*}{$\mathrm{N}_{2}$} & \multirow{8}{*}{$\begin{array}{l}\text { Suncor coker } \\
\text { feed bitumen }\end{array}$} & \multirow{4}{*}{4} & 25 & 0.0180 & \multirow{8}{*}{$\begin{array}{l}\text { Upreti and Mehrotra } \\
\text { (2002) }\end{array}$} \\
\hline & & & 50 & 0.0513 & \\
\hline & & & 75 & 0.2335 & \\
\hline & & & 90 & 0.4960 & \\
\hline & & \multirow{4}{*}{8} & 25 & 0.0555 & \\
\hline & & & 50 & 0.1717 & \\
\hline & & & 75 & 0.4649 & \\
\hline & & & 90 & 0.7460 & \\
\hline
\end{tabular}


Table 2.2 Diffusivities of hydrocarbon solvents in crude oil.

\begin{tabular}{|c|c|c|c|c|c|}
\hline Solvent & Crude oil & $\begin{array}{c}\text { Pressure } \\
{[\mathrm{MPa}]}\end{array}$ & $\begin{array}{c}\text { Temperature } \\
{\left[{ }^{\circ} \mathrm{C}\right]}\end{array}$ & $\begin{array}{l}\text { Diffusivity } \\
{\left[10^{-9} \mathrm{~m}^{2} / \mathrm{s}\right]}\end{array}$ & Reference \\
\hline \multirow{8}{*}{$\mathrm{CH}_{4}$} & $\begin{array}{l}\text { Athabasca } \\
\text { bitumen }\end{array}$ & 4.93 & 50 & $0.40-0.75$ & Schmidt (1989) \\
\hline & $\begin{array}{l}\text { Hamaca } \\
\text { heavy oil }\end{array}$ & 3.42 & 21 & 8.6 & Zhang et al. (2000) \\
\hline & \multirow{6}{*}{$\begin{array}{l}\text { Suncor coker } \\
\text { feed bitumen }\end{array}$} & \multirow{3}{*}{4} & 25 & 0.0810 & \multirow{6}{*}{$\begin{array}{l}\text { Upreti and Mehrotra } \\
\qquad(2002)\end{array}$} \\
\hline & & & 75 & 0.2932 & \\
\hline & & & 90 & 0.4315 & \\
\hline & & \multirow{3}{*}{8} & 25 & 0.0582 & \\
\hline & & & 75 & 0.1518 & \\
\hline & & & 90 & 0.2029 & \\
\hline \multirow{8}{*}{$\mathrm{C}_{2} \mathrm{H}_{6}$} & \multirow{3}{*}{$\begin{array}{l}\text { Athabasca } \\
\text { bitumen }\end{array}$} & \multirow{3}{*}{5} & 20 & 0.1750 & \multirow{3}{*}{ Schmidt (1989) } \\
\hline & & & 50 & 0.1740 & \\
\hline & & & 75 & 0.3370 & \\
\hline & \multirow{5}{*}{$\begin{array}{l}\text { Suncor coker } \\
\text { feed bitumen }\end{array}$} & \multirow{3}{*}{4} & 25 & 0.2539 & \multirow{5}{*}{$\begin{array}{l}\text { Upreti and Mehrotra } \\
\qquad(2002)\end{array}$} \\
\hline & & & 75 & 0.4203 & \\
\hline & & & 90 & 0.6081 & \\
\hline & & \multirow{2}{*}{8} & 75 & 0.4916 & \\
\hline & & & 90 & 0.6917 & \\
\hline
\end{tabular}


mixture from each section of the partition cell was extracted for compositional analysis to determine the amount of dissolved gas. The semi-infinite mass transfer model was then employed to determine the diffusion coefficient from the measured amount of dissolved gas and the gas solubility data. In their mass transfer model, Schmidt et al. (1982) considered bitumen-swelling effect due to gas absorption and assumed the constant diffusivity. The results of their work showed that despite the high viscosity of bitumen, the diffusivity of gases was in the same order of that observed in most liquid systems, i.e., $\sim 10^{-9} \mathrm{~m}^{2} / \mathrm{s}$. Also, it was reported that the diffusivity of carbon dioxide and ethane increases with temperature. Similar results for carbon dioxide, methane and ethane were also reported by Strausz (1989).

Nguyen and Farouq Ali (1998) have determined the diffusivity of carbon dioxide mixed with nitrogen in Aberfeldy heavy oil at $23^{\circ} \mathrm{C}$ and $1 \mathrm{MPa}$. For the compositional analysis, two oil samples were extracted after about 21 days of gas dissolution, one from the top layer and the other from the bottom layer of the saturated heavy oil. It was noted that increasing the concentration of nitrogen in the carbon dioxide stream decreases the solubility and diffusivity of carbon dioxide in the heavy oil.

\subsubsection{Other methods}

Fu and Phillips (1979) introduced a new technique for the determination of diffusivities of volatile hydrocarbon solvent in semi-solid bitumen obtained from Great Canadian Oil Sands Ltd. This technique involves the diffusion of hydrocarbon from a homogenous solution of bitumen-hydrocarbon solvent mixture into a flowing stream of nitrogen, i.e., solute volatilization, which is controlled by the mass transfer resistance of 
the solution while the gas phase resistance is negligible. The diffusivity of the solute was assumed to be independent of concentration and calculated by using a semi-infinite mass transfer model. Their studies showed that the diffusivity of hydrocarbon gas in bitumen decreases with increase in its molecular weight and branching of molecules. Also, the diffusivity becomes smaller in the presence of molecules having ring-shaped structures. The calculated diffusivity values for toluene are two orders larger than those reported for other hydrocarbon liquids (Oballa and Butler, 1989).

Denoyelle and Bardon (1984) measured the diffusivity of carbon dioxide in stock tank oil in the temperature range $66-80^{\circ} \mathrm{C}$ at $15 \mathrm{MPa}$. In their analysis, the volume of the gas dissolved in oil at constant temperature and pressure was measured. The semi-infinite mass transfer model was then utilized to determine the concentration-independent gas diffusivity.

$\mathrm{Yu}$ (1984) used a high-pressure diffusion cell and an optical system to determine the molecular diffusivities of carbon dioxide in $n$-hexadecane at different pressures. The refractive index gradient in the gas-liquid mixture was determined by using the optical system and carbon dioxide concentrations at several liquid depths were related to the measured refractive indices. Molecular diffusivity of the gas-liquid system was then computed by using an existing mathematical diffusion model available in the literature (Duda and Vrentas, 1982). This diffusion model is valid for the system whose diffusivity is dependent on the solvent concentration and whose mixture volume changes significantly during diffusion. The determined diffusivity was also found to increase with increase in system pressure. However, the volume change of $n$-hexadecane during the diffusion is insignificant. 
Renner (1988) developed a method for measuring the molecular diffusion coefficients of $\mathrm{CO}_{2}$, methane and ethane in consolidated porous media saturated with $n$ decane at a high pressure. In this method, the injection solvent is made in contact with $n$ decane in a closed high-pressure cell. As the time passes, the solvent diffuses into the $n$ decane filled pores of the porous media and the pressure in the gas phase tends to decay extremely slowly. Subsequently, the gas is injected automatically so as to maintain constant pressure throughout the diffusion process and the amount of gas injected is recorded with the time. Thus, the obtained volume-time data were utilized to determine the diffusion coefficients of $\mathrm{CO}_{2}$, methane and ethane in $n$-decane at the pressures up to $5.86 \mathrm{MPa}$ and $T=311 \mathrm{~K}$. This technique does not require any visual observations and compositional analyses. However, in the mass transfer model, the solvent-n-decane interface is assumed to be saturated instantaneously, i.e., the interfacial resistance to transport of the gas into the liquid is neglected.

Grogan et al. (1988) determined the diffusivity of carbon dioxide in Maljamar crude oil at $25^{\circ} \mathrm{C}$ and $5.2 \mathrm{MPa}$ through a method based on oil swelling due to gas absorption inside a glass capillary tube. They measured the motion of the gas-oil interface, which can be related to the mass of gas absorbed in the crude oil and employed a semi-infinite mass transfer model to calculate the gas diffusivity. At a higher pressure of 6.9 $\mathrm{MPa}$, experimental measurement became impossible due to enhanced turbulence at the interface and severe asphaltene precipitation.

Oballa and Butler (1989) used infrared radiation to determine the concentration distribution of toluene in the bitumen phase during the diffusion process. The diffusivity was calculated by using a semi-infinite mass transfer model. In addition, the diffusivity of 
toluene was found to be a unimodal function of its concentration in bitumen and to increase from $0.05 \times 10^{-9} \mathrm{~m}^{2} / \mathrm{s}$ at infinite dilution of toluene to a peak value of about $0.44 \times 10^{-9} \mathrm{~m}^{2} / \mathrm{s}$ at 0.4 volume fraction of toluene. The diffusivity then reduced to $0.1 \times 10^{-9}$ $\mathrm{m}^{2} / \mathrm{s}$ at unit volume fraction of toluene.

Riazi and Whitson (1993) proposed a generalized correlation between viscosity and density and used the Stokes-Einstein equation to predict the binary diffusion coefficient. The correlation can be used for both gases and liquids up to a pressure of about $40 \mathrm{MPa}$. The input data for the correlation are molecular weight, critical properties, acentric factor of each component in the system, molar density of mixture, low-pressure and highpressure gas viscosities. The latter three properties have to be calculated from their respective correlations. Thus, the Riazi-Whitson correlation between viscosity and density can be applied to the system with known components. As the heavy oil consists of a large number of hydrocarbon components, nevertheless, this correlation cannot be used to determine the diffusion coefficient of gas solvent in heavy oil.

The diffusivities of propane and butane in the Peace River bitumen were estimated by Das and Butler (1996), based on their VAPEX experiments. By assuming the gas diffusivity as well as the gas-bitumen mixture density to be constant, they empirically correlate the motion of vapour-bitumen interface with the gas diffusivity and the corresponding bitumen viscosity. Their results indicate that the diffusivities of propane and butane are not inversely proportional to their viscosities. Instead, the exponent is close to -0.5 . Also, this method is suitable for highly viscous heavy oil/bitumen system, which is considered virtually immobile in the absence of the dissolved solvent. 


\subsection{Pressure Decay Method}

Riazi (1996) developed the so-called pressure decay method for measuring the diffusion coefficient of a gas in a liquid and tested with methane- $n$-pentane system. This non-intrusive experimental method is based on the fact that the pressure in the gas phase decays as the molecular diffusion of gas into the liquid proceeds in a closed diffusion cell. Such a diffusion process continues until the liquid phase is completely saturated with the gas phase. The gas concentration distribution in the bulk liquid phase is predicted from the analytical solution to the diffusion equation. Further, the diffusivity of a gas in liquid is determined by relating it to the mole fraction of the gas in the liquid with the Sigmund correlation (Sigmund, 1976) or the Riazi-Whitson correlation (Riazi and Whitson, 1993) for binary dense fluids. This method is limited to pure components only because the diffusivity determination is carried out by using either one of the two correlations, which are valid for pure components.

By means of the pressure decay method, Zhang et al. (2000) measured the diffusivity of carbon dioxide in heavy oil at $2.84 \mathrm{MPa}$ and $21^{\circ} \mathrm{C}$ and the diffusivity of methane in heavy oil at $3.42 \mathrm{MPa}$ and $21^{\circ} \mathrm{C}$, respectively. The diffusivities of both the gas solvents in heavy oil (See Tables 2.1 and 2.2) were determined through a mass transfer model coupled with the conservation of mass in the closed diffusion cell. The experimental pressure decay for carbon dioxide-heavy oil system was measured for 21 days, whereas the pressure decay for methane-heavy oil system was recorded for 5 days. The so-called history matching (e.g., non-linear regression) was carried out to find the best fit of the measured pressure decay data with the predicted pressure decay results by using the diffusion coefficient as an adjustable parameter. In their calculation, they 
assumed constant compressibility factor for gas, no resistance to mass transfer at the solvent-heavy oil interface, no volume change of heavy oil phase (i.e., no oil swelling effect), no chemical reactions, concentration-independent diffusivity and non-volatile heavy oil.

By using the similar approach, Upreti and Mehrotra $(2000 ; 2002)$ improved the pressure decay method to measure the concentration-dependent diffusivities of carbon dioxide, methane, ethane and nitrogen in bitumen in the temperature range of $25-90^{\circ} \mathrm{C}$ at 4 and $8 \mathrm{MPa}$, respectively. These gas diffusivities were formulated as a function of temperature and gas mass fraction in bitumen (Upreti, 2000). Their mass transfer model utilized the numerical matching of calculated mass of gas dissolved into bitumen with the experimental value by using the diffusion coefficient as an adjustable parameter at a particular depth and time. The concentration-averaged diffusivities were also obtained in their studies and are given in Tables 2.1 and 2.2.

Later, Civan and Rasmussen $(2001 ; 2002 ; 2003)$ developed a transient state nonequilibrium diffusion model to determine the gas diffusion coefficient from the experimental data available in the literature (Riazi, 1996; Sachs, 1998; Zhang et al., 2000). Both the finite and semi-infinite analytical solutions of this model for the diffusion process were developed. These two solutions were then reformulated to determine the diffusion coefficient by means of regression of the resultant analytical expressions to the experimental data. This transient non-equilibrium diffusion model accounts for the interfacial resistance to gas mass transfer into heavy oil and assumes no volume change of heavy oil, no chemical reactions, concentration-independent diffusion coefficient, and non-volatile heavy oil. 


\subsection{Problem Statement}

As described in the preceding section, some major improvements of the pressure decay method have been made in the past to determine the diffusion coefficient of a solvent in heavy oil/bitumen. Nevertheless, both the experiment and the numerical modeling can be further improved so as to apply this method for a wide variety of solvents.

During the experiment, the decaying pressures should be measured accurately and continuously for a sufficiently long time, as the accuracy of the determined diffusion coefficient depends on that of measured pressures. Therefore, this study is aimed at measuring the decaying pressures accurately by properly constructing the experimental setup. The first two solvents that are tested in this thesis study are carbon dioxide and methane.

In general, a variety of solvents are used in the solvent-based recovery processes. Diffusion coefficient data for some hydrocarbon and non-hydrocarbon solvents in some specific heavy oil/bitumen are made available in the literature (See Tables 2.1 and 2.2). However, the measured diffusion coefficient for propane in heavy oil is not available, except that there are some correlations (Das and Butler, 1998). As the diffusivity for such a solvent-heavy oil is vital in designing the VAPEX process, it will also be measured in this thesis study by using the pressure decay method.

With respect to the diffusion model, there exists no consensus on what boundary condition should be applied at the solvent-heavy oil interface. For example, the heavy oil at the interface was assumed to be saturated with the solvent under the so-called equilibrium pressure at all times (Zhang et al., 2000; Renner, 1988), which is termed as 
the equilibrium boundary condition (BC). On the other hand, Riazi (1996) and Upreti and Mehrotra $(2000 ; 2002)$ assumed that the solvent concentration at the interface is equal to the saturation concentration (i.e., solubility), which varies with the existing pressure in the solvent phase during the experiment. This interface boundary condition is called the quasi-equilibrium BC. Finally, Civan and Rasmussen $(2001 ; 2002 ; 2003)$ applied the socalled non-equilibrium $\mathrm{BC}$ at the solvent-heavy oil interface, which considers the interface mass transfer resistance. Thus, the above-mentioned three BCs are applied for the three solvent-heavy oil systems to find the most suitable BC for each system.

It should also be noted that, in the past, all the diffusion experiments were conducted for very long time with an attempt to reach the equilibrium pressure so that the saturation concentration (or solubility) of solvent in heavy oil can be determined. In this study, however, a novel strategy is adopted to determine the equilibrium pressure for each solvent-heavy oil system. Hence, as an important improvement of the pressure decay method, in this study, the diffusion coefficient for each system is determined by using the pressure decay data measured in a short period. 


\section{Chapter 3 THEORY}

\subsection{Interface Mass Transfer Models}

\subsubsection{Diffusion equation}

When a solvent and a heavy oil sample are made in contact with each other inside a closed diffusion cell, the solvent gradually dissolves in heavy oil until the latter is completely saturated with the former. A schematic of the solvent-heavy oil system inside a closed diffusion cell is shown in Fig. 3.1, where the bottom of the pressure cell is located at $x=0$ and the solvent-heavy oil interface is located at $x=L$. By applying the continuity equation and the Fick's first law, the molecular diffusion process in such a solvent-heavy oil system can be described by an unsteady one-dimensional diffusion equation (Bird et al., 2002):

$$
\frac{\partial c}{\partial t}=D \frac{\partial^{2} c}{\partial x^{2}}
$$

where $c(x, t)$ is the molar concentration of solvent in the heavy oil; $x$ is the distance from the bottom of the diffusion cell; $t$ is time; and $D$ is the diffusion coefficient of solvent in heavy oil, assuming that it is constant throughout the diffusion process. In the above equation, the natural convection is not accounted for because the density of the heavy oil near the interface is lower (i.e., the so-called oil swelling effect) than that of the heavy oil at the bottom of the diffusion cell during the diffusion process. Therefore, the mass transfer of solvent into heavy oil occurs only due to molecular diffusion. In the experiment, the position of the solvent-heavy oil interface does not change appreciably and thus the oil swelling effect can be neglected (Zhang et al., 2000). In addition, only 


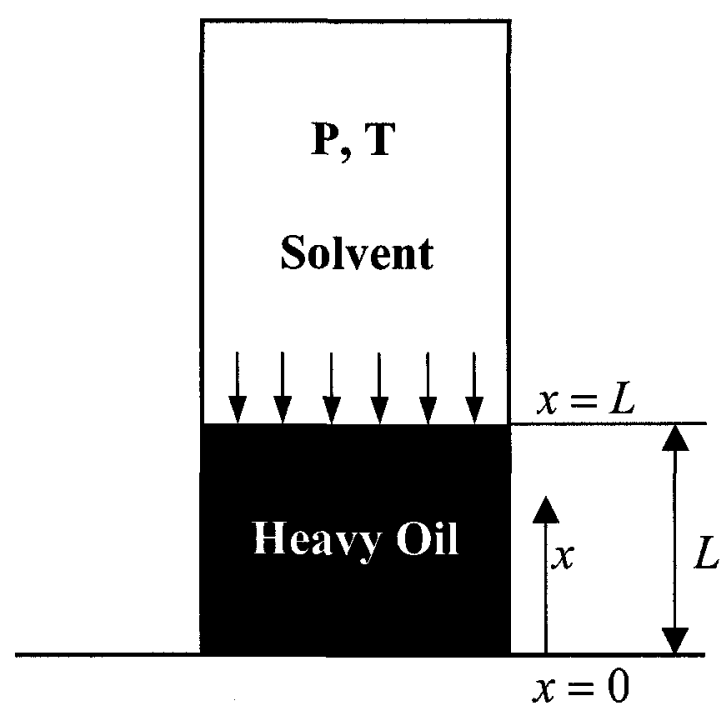

Figure 3.1 Schematic of solvent-heavy oil system inside a closed diffusion cell. 
one-way diffusion of pure solvent into heavy oil is considered because the latter can be usually assumed to be non-volatile under the test conditions. The overall diffusion process is also assumed to be isothermal.

\subsubsection{Initial condition and boundary conditions}

The specific solution to the above diffusion equation depends upon the initial condition (IC) and the boundary conditions (BCs). At the beginning of the test, the concentration of solvent in the heavy oil phase is equal to zero. Thus, the initial condition is given by:

$$
\left.c(x, t)\right|_{t=0}=0 \quad 0 \leq x \leq L .
$$

For the impermeable rigid boundary at the bottom of the diffusion cell, the mass transfer flux at any time is equal to zero (Crank, 1975). Therefore, the Neumann boundary condition can be applied at the bottom of the diffusion cell:

$$
\left.\frac{\partial c}{\partial x}\right|_{x=0}=0 \quad t>0
$$

The boundary condition at the solvent-heavy oil interface is also required in formulating sufficient mathematical description of the concentration distribution of solvent in the heavy oil. In principle, three different BCs can be applied at the solventheavy oil interface, namely, the equilibrium $\mathrm{BC}$, quasi-equilibrium $\mathrm{BC}$ and nonequilibrium BC (Tharanivasan et al., 2003). These three different BCs will be explained in detail in the following paragraphs. Consequently, in this study, the diffusion equation in Eq. [3.1] subject to the initial condition in Eq. [3.2], the boundary condition at the bottom of the diffusion cell in Eq. [3.3], and one of the three BCs at the solvent-heavy oil interface, is referred to as an interface mass transfer model. 
As the first type of $\mathrm{BC}$ at the solvent-heavy oil interface, the equilibrium boundary condition assumes that the interface is saturated with the solvent under the so-called equilibrium pressure at all times (Renner, 1988; Zhang et al., 2000). The corresponding Dirichlet boundary condition at the solvent-heavy oil interface is written as:

$$
\left.c(x, t)\right|_{x=L}=c_{\text {sat }}\left(P_{e q}\right) \quad t>0 .
$$

Here, $P_{\text {eq }}$ is the pressure in the solvent phase when the heavy oil is completely saturated or in equilibrium with the solvent, which is called the equilibrium pressure; and $c_{\text {sat }}\left(P_{\text {eq }}\right)$ is termed the solvent saturation concentration or solubility of the solvent in heavy oil under the equilibrium pressure, $P_{\text {eq }}$.

In the quasi-equilibrium boundary condition (Riazi, 1996; Upreti and Mehrotra, $2000 ; 2002$ ), which is the second type, the solvent-heavy oil interface is assumed to be saturated with the solvent under the existing pressure in the solvent phase rather than the equilibrium pressure. Therefore, the respective Dirichlet boundary condition is expressed as:

$$
\left.c(x, t)\right|_{x=L}=c_{\text {sat }}[P(t)] \quad t>0,
$$

where $P(t)$ is the existing pressure in the solvent phase, which decays with time during the diffusion process until the equilibrium pressure $P_{\mathrm{cq}}$ is reached.

The so-called non-equilibrium boundary condition is the third type (Civan and Rasmussen, 2001; 2002; 2003). In this boundary condition, the solvent mass transfer flux at the solvent-heavy oil interface is assumed to be proportional to the difference between the solvent saturation concentration under the equilibrium pressure and the existing solvent concentration at the interface. By applying the Fick's first law, the corresponding Robin boundary condition is given by: 


$$
\left.D \frac{\partial c}{\partial x}\right|_{x=L}=k\left[c_{s a t}\left(P_{e q}\right)-\left.c(x, t)\right|_{x=L}\right] \quad t>0
$$

where, $k$ is the mass transfer coefficient at the solvent-heavy oil interface, and $1 / k$ represents the interfacial resistance to the mass transfer across the interface.

\subsubsection{Non-dimensionalization}

To solve the diffusion equation [3.1], subject to the initial condition in Eq. [3.2], the boundary condition at the bottom of the diffusion cell in Eq. [3.3] and one of the three BCs at the solvent-heavy oil interface in Eqs. [3.4a-c], it is better to express all the equations in a dimensionless form. Equations [3.1] through [3.4] can be nondimensionalized by introducing the following dimensionless variables and parameter:

$$
C=\frac{c}{c_{s a t}\left(P_{e q}\right)}, \quad X=\frac{x}{L}, \quad \tau=\frac{t}{L^{2} / D}, \quad k_{D}=\frac{k L}{D} .
$$

Here, $\tau$ is the dimensionless time, which is also called the mass-transfer Fourier number; and $k_{\mathrm{D}}$ can be referred to as the mass-transfer Biot number, which represents the ratio of the bulk resistance to the mass transfer due to the molecular diffusion, $L / D$, to the interfacial resistance to the mass transfer, $1 / k$. Thus, the corresponding diffusion equation [3.1], the initial condition in Eq. [3.2], and the boundary condition at the bottom of the diffusion cell in Eq. [3.3] can be expressed in terms of the dimensionless variables:

$$
\begin{array}{cc}
\frac{\partial C}{\partial \tau}=\frac{\partial^{2} C}{\partial X^{2}}, \\
\left.C(X, \tau)\right|_{\tau=0}=0 & 0 \leq X \leq 1, \\
\left.\frac{\partial C}{\partial X}\right|_{X=0}=0 & \tau>0 .
\end{array}
$$


Likewise, the BCs at the solvent-heavy oil interface in Eqs. [3.4a-c] in terms of the dimensionless variables and parameter become:

$$
\begin{array}{ll}
\left.C(X, \tau)\right|_{X=1}=1 & \tau>0, \\
\left.C(X, \tau)\right|_{X=1}=\frac{c_{\text {sat }}[P(\tau)]}{c_{\text {sat }}\left(P_{e q}\right)} & \tau>0, \\
\left.\frac{\partial C}{\partial X}\right|_{X=1}=k_{D}\left[1-\left.C(X, \tau)\right|_{X=1}\right] & \tau>0 .
\end{array}
$$

\subsubsection{Analytical solutions}

Analytical solution to the diffusion equation in Eq. [3.6] can be obtained, subject to the initial condition in Eq. [3.7], the boundary condition at the bottom of the diffusion cell in Eq. [3.8] and one of the three BCs at the solvent-heavy oil interface in Eqs. [3.9ac]. There are two standard forms of the analytical solutions (Crank, 1975). The first form consists of a series of trigonometric functions that converge satisfactorily at large times, i.e., close to the quasi-steady state. This form of analytical solution is derived particularly for diffusion in a finite medium. The second form comprises of a series of error functions and is more suitable for numerical predictions at small times, i.e., at the early stage of transient diffusion process. In general, the error function solution is derived for diffusion in a semi-infinite or an infinite medium. As the diffusion of solvent into heavy oil proceeds rather slowly and the diffusion system studied here is of finite medium, the first form of the analytical solutions consisting of a series of trigonometric functions is invoked. It is worthwhile to point out that, if this type of solution is pursued, more terms should be included in calculating the solvent concentration so as to attain desired accuracy at the early stage of transient diffusion process. In this study, the standard type 
of the analytical solutions consisting of a series of trigonometric functions is used for all the three BCs and obtained by the separation of variables method.

If the equilibrium $\mathrm{BC}$ in Eq. [3.9a] is applied at the solvent-heavy oil interface together with the initial condition in Eq. [3.7] and the $\mathrm{BC}$ at the bottom of the diffusion cell in Eq. [3.8], the specific analytical solution to the diffusion equation in Eq. [3.6] is expressed as:

$$
C(X, \tau)=1-2 \sum_{n=0}^{\infty}(-1)^{n} \frac{\cos \left[\frac{(2 n+1) \pi}{2} X\right]}{\frac{(2 n+1) \pi}{2}} e^{-\frac{(2 n+1)^{2} \pi^{2}}{4} \tau}
$$

The dimensional form of this analytical solution to the diffusion equation with such IC and BCs was previously given by Crank (1975). The detailed derivation of this analytical solution in a dimensionless form is given in Appendix A.1.

In order to obtain a semi-analytical solution to the diffusion equation in Eq. [3.6] together with the initial condition in Eq. [3.7], the BC at the bottom of the diffusion cell in Eq. [3.8], and the quasi-equilibrium BC in Eq. [3.9b] at the solvent-heavy oil interface, it is assumed that the solvent saturation concentration in the heavy oil is proportional to the pressure at the interface (Mehrotra et al., 1992). Hence, Eq. [3.9b] becomes:

$$
\left.C(X, \tau)\right|_{X=1}=\frac{P(\tau)}{P_{e q}} \quad \tau>0
$$

Therefore, the semi-analytical solution for this case can be obtained only if the measured pressure decay data are explicitly expressed as a simple exponential function of time. The best-fit curve to the measured decaying pressures for a solvent-heavy oil system was formulated by Zhang et al. (2000): 


$$
P(t)=m_{1} e^{-\frac{t}{k_{1}}}+m_{2} e^{-\frac{t}{k_{2}}}+P_{e q},
$$

where the specific values of $m_{1}, m_{2}, k_{1}$ and $k_{2}$ for a given solvent-heavy oil system can be found by using the non-linear regression. The above equation can be nondimensionalized by applying Eq. [3.5] and introducing the following dimensionless parameters:

$$
\alpha=\frac{m_{1}}{P_{e q}}, \beta=\frac{m_{2}}{P_{e q}}, \kappa_{1}=\frac{k_{1}}{L^{2} / D}, \kappa_{2}=\frac{k_{2}}{L^{2} / D},
$$

where $\alpha$ and $\beta$ are the dimensionless pressures; $\kappa_{1}$ and $\kappa_{2}$ are the dimensionless times. Thus, the semi-analytical solution to the diffusion equation in Eq. [3.6] corresponding to the quasi-equilibrium BC in Eq. [3.11] can be derived from the general solution given by Carslaw and Jaeger (1959). This solution is further expressed in terms of the dimensionless variables and parameters defined in Eqs. [3.5] and [3.13]:

$$
\begin{gathered}
C(X, \tau)=\sum_{n=0}^{\infty}(-1)^{n}(2 n+1) \pi \cos \left[\frac{(2 n+1) \pi}{2} X\right]\left\{\frac{4 \kappa_{1} \alpha}{(2 n+1)^{2} \pi^{2} \kappa_{1}-4}\left[e^{-\frac{\tau}{\kappa_{1}}}-e^{-\frac{(2 n+1)^{2} \pi^{2}}{4} \tau}\right]\right. \\
\left.+\frac{4 \kappa_{2} \beta}{(2 n+1)^{2} \pi^{2} \kappa_{2}-4}\left[e^{-\frac{\tau}{\kappa_{2}}}-e^{-\frac{(2 n+1)^{2} \pi^{2}}{4} \tau}\right]+\frac{4}{(2 n+1)^{2} \pi^{2}}\left[1-e^{-\frac{(2 n+1)^{2} \pi^{2}}{4} \tau}\right]\right\} \cdot[3.14]
\end{gathered}
$$

For the non-equilibrium $\mathrm{BC}$ in Eq. [3.9c] at the solvent-heavy oil interface, the specific solution to the diffusion equation in Eq. [3.6] was derived by Walas (1991) and its dimensionless form is obtained as:

$$
C(X, \tau)=1-2 \sum_{n=1}^{\infty} \frac{\sin \lambda_{n}}{\left(\lambda_{n}+\sin \lambda_{n} \cos \lambda_{n}\right)} \cos \left(\lambda_{n} X\right) e^{-\lambda_{n}^{2} \tau},
$$


where $\lambda_{n}(n=1,2,3, \ldots)$ represent the eigen values, which are the positive roots of the characteristic equation, $\tan \lambda_{n}=k_{D} / \lambda_{n}$, and depend solely on the mass-transfer Biot number, $k_{\mathrm{D}}$. The detailed procedure in attaining this analytical solution in a dimensionless form is given in Appendix A.2. Furthermore, in this study, the bisection method is used to find the eigen values numerically. In the determination of each eigen value by using this numerical method (Akai, 1994), the upper and lower limits of the positive roots are chosen as $(n-1) \pi$ and $(n-1) \pi+\pi / 2$, respectively. The computation of a root is continued until the difference between the successive roots is less than $1.0 \times 10^{-12}$.

It should be pointed out that the analytical solution given in Eq. [3.15] for the nonequilibrium $\mathrm{BC}$ in Eq. [3.9c] becomes invalid when $k_{\mathrm{D}}=0$. This may occur only in two hypothetical cases. The first case happens if solvent does not dissolve in the heavy oil. Therefore, the solvent concentration in heavy oil is always equal to zero. Apparently, this is a trivial case. The second case occurs when the diffusion coefficient $D$ is infinite and/or the height $L$ of the heavy oil sample in the diffusion cell is infinitesimal. Again this case is unrealistic. In reality, the mass-transfer Biot number, $k_{D}=\frac{k L}{D}$, can be approximated as zero only if the diffusion coefficient is sufficiently large and/or the height of the heavy oil is sufficiently small. Physically, $k_{\mathrm{D}} \approx 0$ means that the interfacial resistance to the mass transfer, $1 / k$, is much larger than the bulk resistance to the molecular diffusion, $L / D$. Consequently, the solvent concentration in the bulk heavy oil phase is almost uniform. This uniform concentration $c_{u}$ for $k_{\mathrm{D}}=0$ is governed by the following ordinary differential equation:

$$
L \frac{d c_{u}}{d t}=k\left[c_{s a t}\left(P_{e q}\right)-c_{u}\right]
$$


The corresponding analytical solution is given by:

$$
c_{u}=c_{s a t}\left(P_{e q}\right)\left[1-e^{-\frac{k t}{L}}\right] .
$$

The above solution is used to determine the uniform solvent concentration for $k_{\mathrm{D}}=0$.

It is worthwhile to note that, in the calculation of the dimensionless solvent concentration distribution for each of the three interface mass transfer models, the number of terms that should be taken into account in the infinite series depends on the pre-specified accuracy. Here, the accuracy represents the magnitude of the first term in the truncated series. In this work, calculation of the dimensionless solvent concentration distribution continues until the difference between the consecutive concentrations is less than $1.0 \times 10^{-9}$.

\subsection{Calculated Pressure Decay Curves}

Once the dimensionless solvent concentration distribution in the heavy oil phase is obtained, the calculated pressure, $P_{\text {cal }}(t)$, in the solvent phase at any time can be determined by following the general procedure as described below. First, the total number of moles of solvent dissolved in the heavy oil sample is computed by numerically integrating the dimensional solvent concentration distribution for each interface mass transfer model. Here, the volume of heavy oil phase is assumed to be constant during the diffusion process, i.e., the oil swelling effect is not considered in this study. This assumption may be valid because the position of the interface does not change appreciably during the experiment (Zhang et al., 2000). The trapezoidal method of numerical integration is used and the integral interval of the dimensionless distance is chosen to be $\Delta X=0.005$, based on the following sensitivity analysis. The differences 
between the dimensionless solvent concentrations calculated by using $\Delta X=0.005$ and $\Delta X$ $=0.001$ are found to be less than $0.01 \%$ for large times, whereas they are approximately $0.2 \%$ for small times. Therefore, the integral interval of $\Delta X=0.005$ is small enough to obtain the accurate numerical results. The resultant quantity $N_{\mathrm{o}}(t)$ of the above numerical integration represents the total number of moles of solvent dissolved in the heavy oil.

Then, in terms of the solvent mass conservation in the closed system, the number of moles of solvent remaining in the gas phase at any time is equal to $N_{\mathrm{g}}(t)=N_{\mathrm{i}}-N_{\mathrm{o}}(t)$. Here, $N_{\mathrm{i}}$ represents the initial total number of moles in the solvent phase, which is calculated from the initial pressure by using an equation of state (EOS) for a real gas. Hence, if $N_{\mathrm{g}}(t)$ is known, the pressure in the solvent phase $P_{\text {cal }}(t)$ can be calculated from the EOS for a real gas:

$$
P_{\text {cal }}(t) V_{\text {solvent }}=Z\left(P_{\text {cal }}(t), T\right) N_{\mathrm{g}}(t) R T=Z\left(P_{\text {cal }}(t), T\right)\left[N_{\mathrm{i}}-N_{\mathrm{o}}(t)\right] R T,
$$

where, $V_{\text {solvent }}$ is the volume of solvent phase in the diffusion cell; $Z\left(P_{\text {cal }}(t), T\right)$ is the compressibility factor or simply the Z-factor, which is a function of pressure $P_{\text {cal }}(t)$ and the absolute temperature $T$; and $R$ is the universal gas constant.

In this study, the Z-factor in the above EOS is determined by using the Lee-Kesler correlation (Lee and Kesler, 1975). Prior to using this correlation, the Z-factor is also calculated (Tharanivasan et al., 2004a; 2004b) by using the Hall-Yarborough equation (Hall and Yarborough, 1973), which is the most accurate representation of the StandingKatz Z-factor correlation chart (Standing and Katz, 1942). For both carbon dioxide and methane, Z-factors calculated by using the Lee-Kesler correlation and Hall-Yarborough equation are almost same. However, the Hall-Yarborough equation is not recommended when the reduced temperature of the solvent is much greater than 1.0 (Yarborough and 
Hall, 1974). Due this reason, the Z-factor for propane is not calculated by using the HallYarborough equation. Instead, the Lee-Kesler correlation is used to calculate the Zfactors for the three solvents tested in this study (Tharanivasan et al., 2004c). The complete description on how to calculate the Z-factor is given in Appendix B.1 when the Lee-Kesler correlation is invoked, and in Appendix B.2 if the Hall-Yarborough equation is used.

\subsection{Determination of Equilibrium Pressure}

In the above-mentioned three interface mass transfer models, the equilibrium pressure, $P_{\text {eq }}$, is always required to determine the saturation concentration, $c_{\mathrm{sat}}\left(P_{\mathrm{eq}}\right)$, of solvent in heavy oil. Ideally, the equilibrium pressure can be measured when the heavy oil is completely saturated with the solvent. In practice, however, such a slow diffusion process may take an unreasonably long time to reach the equilibrium state. Alternatively, in this study, the equilibrium pressure, $P_{\text {eq }}$, is calculated from the measured solubility data for the same solvent-heavy oil system. At the equilibrium state, the EOS for the solvent phase, Eq. [3.18a], can be rewritten as follows.

$$
P_{\text {eq }} V_{\text {solvent }}=Z\left(P_{\text {eq }}, T\right) N_{\text {eq }} R T=Z\left(P_{\text {eq }}, T\right)\left[N_{i}-\chi\left(P_{\text {eq }}, T\right) \frac{m_{\text {oil }}}{M_{\text {solvent }}}\right] R T,
$$

where $Z\left(P_{\text {eq }}, T\right)$ is the $Z$-factor and $\chi\left(P_{\text {eq }}, T\right)$ is the solubility of a solvent in heavy oil at the equilibrium pressure $P_{\text {eq }}$ and at a given temperature $T ; N_{\text {eq }}$ denotes the number of moles of solvent remaining in the gas phase at the equilibrium pressure, $P_{\mathrm{eq}} ; m_{\mathrm{oil}}$ is the mass of heavy oil inside the closed diffusion cell and $M_{\text {solvent }}$ is the molecular weight of solvent tested. In the experiment, $V_{\text {solvent, }} m_{\mathrm{oil}}$ and $T$ are known, and $N_{\mathrm{i}}$ can be calculated from the initial pressure by applying Eq. [3.18a] at the very beginning. In this study, $Z\left(P_{\mathrm{eq}}, T\right)$ is 
determined from the Lee-Kesler correlation (Lee and Kesler, 1975), while $\chi\left(P_{\text {eq }}, T\right)$ represents the measured solubility data for the same solvent-heavy oil system. It should be noted that, in Eq. [3.18b], because both the Z-factor and the solubility, $Z\left(P_{\text {eq }}, T\right)$ and $\chi\left(P_{\mathrm{eq}}, T\right)$, are functions of the equilibrium pressure $P_{\mathrm{eq}}$ at a given temperature $T$, the equilibrium pressure $P_{\text {eq }}$ has to be computed by using the iteration method. The iteration process continues until the relative difference between the consecutive equilibrium pressures is less than $0.01 \%$. A FORTRAN program written for determining the equilibrium pressure from the measured solubility data is given in Appendix C.1.

\subsection{Numerical Optimization}

\subsubsection{Objective function}

After the pressure vs. time curve, i.e., $P_{\text {cal }}(t)$, is calculated from Eq. [3.18a] for each of the three $\mathrm{BCs}$, the true diffusion coefficient $D$ can be determined by finding the best match between the theoretically calculated pressure-time curve $P_{\text {cal }}(t)$ and the experimentally measured pressure-time data $P_{\exp }(t)$. By using the diffusion coefficient as an adjustable parameter, the so-called history matching technique is applied to minimize an objective function $\Delta P_{\text {ave }}$, which is defined as:

$$
\Delta P_{\mathrm{ave}}=\sqrt{\frac{\sum_{i=1}^{m}\left|P_{\mathrm{cal}}(t)-P_{\mathrm{exp}}(t)\right|_{t=t_{i}}^{2}}{m}}=\sqrt{\frac{\sum_{i=1}^{m}\left|\Delta P_{i}\right|^{2}}{m}} .
$$

Here, the difference between the calculated pressure $P_{\text {cal }}(t)$ and the measured pressure $P_{\text {exp }}(t)$ is expressed by $\Delta P_{i}$ at $t=t_{i}, i=1,2,3, \ldots, m$. Mathematically, the average pressure difference $\Delta P_{\text {ave }}$ is defined as an average of all the pressure differences corresponding to a guessing value of diffusion coefficient $D$. Physically, this objective function represents 
the discrepancy between the theoretically calculated and experimentally measured pressure vs. time curves.

\subsubsection{One-parameter simultaneous search method}

More specifically, when the equilibrium $\mathrm{BC}$ or the quasi-equilibrium $\mathrm{BC}$ is applied at the solvent-heavy oil interface, the objective function is minimized by using the diffusion coefficient $D$ as the only adjustable parameter. The minimization scheme is carried out by using the one-parameter simultaneous search method (Husain and Gangiah, 1976). Successful application of this search method depends upon two conditions. First, the lower and upper limits (i.e., the initial interval of uncertainty) of the adjustable parameter should be known, between which the optimal $D$ is located so that the objective function is minimized. Secondly, the objective function should be unimodal. In this study, the lower and upper limits of the diffusion coefficient are chosen as $0.01 \times 10^{-9}$ and $100 \times 10^{-9} \mathrm{~m}^{2} / \mathrm{s}$, respectively, which should cover most practical cases of interest. Also it is found from numerical calculations that the objective function, $\Delta P_{\text {ave }}$, is unimodal with respect to the diffusion coefficient $D$. In this search method, the objective function is first evaluated at ten equally spaced interior trial points. Then the immediate points on both sides of the trial point, at which the minimum objective function is obtained, are chosen as the new limits of interval of uncertainty. This search procedure is repeated until the interval reaches the termination criterion of $0.01 \times 10^{-9} \mathrm{~m}^{2} / \mathrm{s}$ for the diffusivity $D$. The average of the lower and upper limits of the final interval is called the optimal $D$ and considered as the true diffusion coefficient for the equilibrium $\mathrm{BC}$ or the quasi-equilibrium BC. FORTRAN programs for determining the diffusion coefficient are 
provided in Appendix C.2 for the equilibrium BC and in Appendix C.3 for the quasiequilibrium $\mathrm{BC}$, respectively.

\subsubsection{Two-parameter simultaneous search method}

If the non-equilibrium $\mathrm{BC}$ is imposed at the solvent-heavy oil interface, the objective function is minimized by using both the diffusion coefficient $D$ and the masstransfer Biot number $k_{\mathrm{D}}$ as the adjustable parameters. In this two-parameter simultaneous search method, the lower and upper limits of $k_{\mathrm{D}}$ are chosen as 0.01 and 500 , whereas the search extremes for $D$ remain the same as those used in the one-parameter simultaneous search method. The initial interval of uncertainty for $k_{\mathrm{D}}$ is divided into ten equally spaced interior trial points. At each trial point, the local optimal diffusion coefficient and the corresponding local minimum objective function are found by using the above-mentioned one-parameter search method. A new interval of uncertainty for $k_{\mathrm{D}}$ is found by comparing the local minimum objective functions at all the trial points. This search procedure is repeated until the interval of uncertainty reaches the termination criterion of 0.01 for the mass-transfer Biot number $k_{\mathrm{D}}$. The corresponding termination criterion for $D$ remains the same as that used in the one-parameter simultaneous search method. Thus, the diffusion coefficient corresponding to the global minimum objective function is called the global optimal $D$ and considered as the true diffusion coefficient for the non-equilibrium BC. By using the two-parameter simultaneous search method, the optimal $k_{\mathrm{D}}$ is also found and considered as the true mass-transfer Biot number for this BC. The determination of the diffusion coefficient and the mass-transfer Biot number for a given solvent-heavy oil system by applying the non-equilibrium $\mathrm{BC}$ at the interface is performed by executing a FORTRAN program given in Appendix C.4. 


\section{Chapter 4 EXPERIMENTAL MEASUREMENTS}

\subsection{Materials}

The heavy oil sample is collected from Well 11/04-33-51-23W3M/2 in the Celtic field, Waseca formation, Lloydminster area, Alberta, Canada, and cleaned by Alberta Research Council (ARC), Edmonton, Canada. The density and viscosity of the cleaned field heavy oil are measured at the Petroleum Technology Research Centre (PTRC) and they are equal to $988 \mathrm{~kg} / \mathrm{m}^{3}$ and $20,267 \mathrm{mPa} \cdot \mathrm{s}$ at 1 atm and $23.9^{\circ} \mathrm{C}$, respectively. The asphaltene content of the heavy oil sample is $11.5 \mathrm{wt} . \% \mathrm{n}$-heptane insoluble. The gaseous solvents used in the experiments, carbon dioxide, methane and propane, have the purity of $99.99 \%, 99.97 \%, 99.5 \%$, respectively. Nitrogen is used for drying and leakage testing and its purity is equal to $99.998 \%$. These gases are purchased from Praxair, USA.

\subsection{Pressure Decay Measurements}

\subsubsection{Experimental setup}

During the course of this thesis study, a complete experimental setup is successfully designed, constructed, tested and used to study the diffusion process of a solvent into the heavy oil sample under high pressures at constant temperature. Figure 4.1 shows a block diagram of the experimental setup used to measure the decaying pressures as the solvent gradually diffuses into heavy oil inside a closed diffusion cell at constant temperature. The experimental apparatus comprises of a stainless steel cylindrical diffusion cell (CYL0250-10NP-316-2, DBR, Canada) of $5.4 \mathrm{~cm}$ inner diameter and $16.0 \mathrm{~cm}$ length, which is fitted with two cylinder caps at the two ends. A Viton O-ring is placed between the 


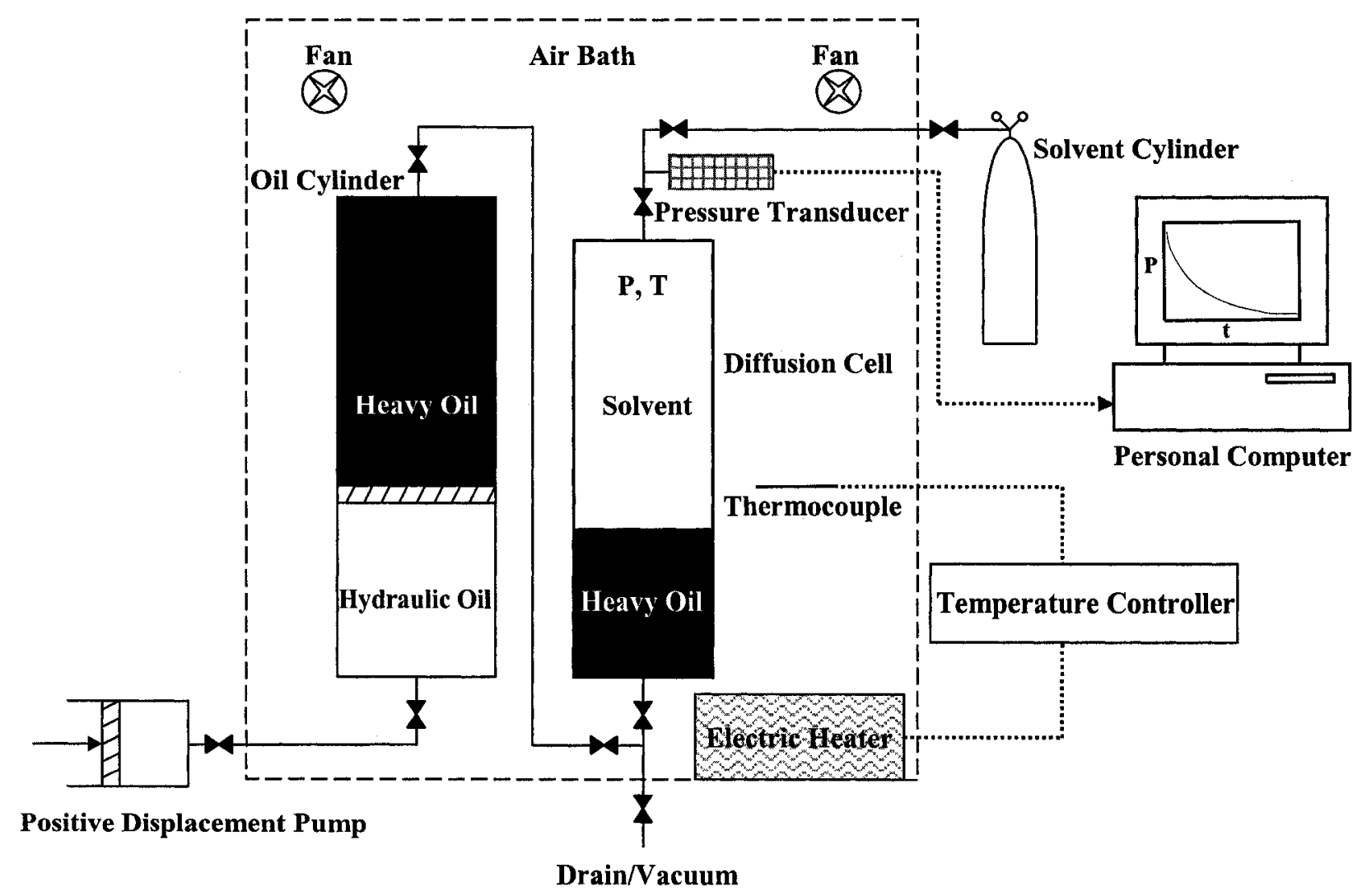

Figure 4.1 Block diagram of the experimental setup used to measure the solvent-heavy oil diffusion coefficient at constant temperature. 
cylinder cap and the diffusion cell at each end and several threaded screws are used to seal the cell. The maximum operating pressure and temperature of the diffusion cell are equal to $69,000 \mathrm{kPa}$ and $200^{\circ} \mathrm{C}$, respectively. The diffusion cell is mounted vertically on a metal support throughout the experiment. The top port is connected to the solvent supply cylinder through a needle valve (SS-0VS2, Swagelok, USA), whereas the bottom port is connected to a heavy oil transfer cylinder. The heavy oil sample is introduced into the diffusion cell by using a high pressure positive displacement pump (PMP-500-1-10HB-316-MO-CO, DBR, Canada). The bottom port is also connected to a needle valve for evacuating the diffusion cell before the experiment or draining the heavy oil sample after the experiment. All the connections in the experimental setup are made by using $1 / 8^{\prime \prime}$ stainless steel tubing (Swagelok, USA).

In addition to the above-mentioned diffusion cell, another main component in the experimental apparatus is a high-precision digiquartz pressure transducer (46KR-101, Paroscientific, Inc., USA), which is connected to the top port of the diffusion cell as shown in Fig. 4.1. The pressure transducer (See Fig. 4.2) is used to measure the absolute pressure in the solvent phase inside the closed diffusion cell with an accuracy of $0.01 \%$ at a full scale of $41,385 \mathrm{kPa}$. It is accurately calibrated and carefully tested by the manufacturer prior to shipping. On-line data acquisition and pressure display are made possible through an interface board and software purchased from the manufacturer. The pressure transducer also has a built-in function of automatically recording and displaying the temperature of the solvent phase in a personal computer.

In order to maintain constant temperature condition (i.e., isothermal diffusion process) throughout the experiment, the whole experimental setup is placed inside an air 


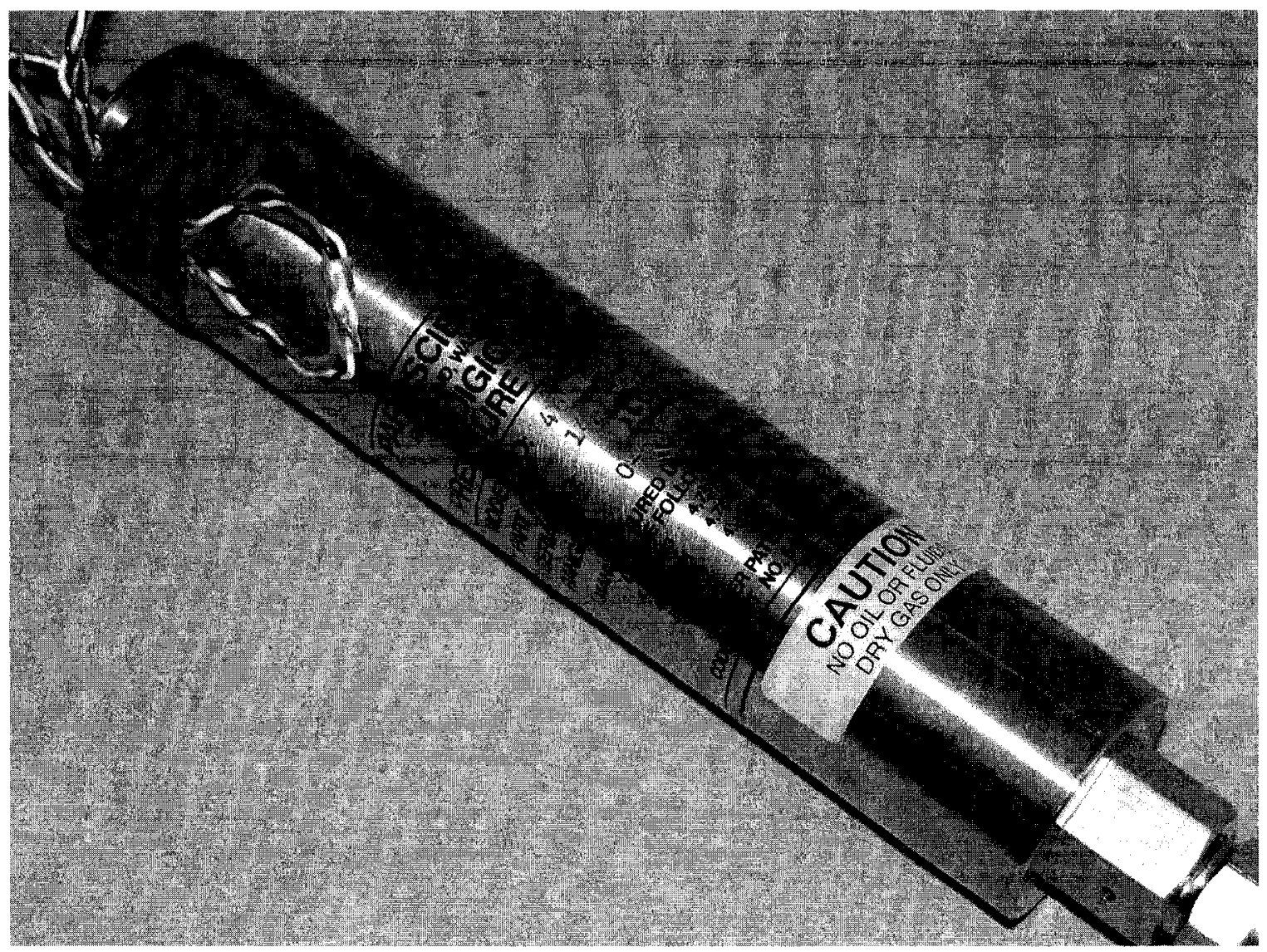

Figure 4.2 High-precision absolute pressure transducer used to measure the decaying pressure in the diffusion cell. 
bath. A temperature controller (Standard-89000-00, Cole-Parmer, Canada) is used to control the heat supply from an electric heater. Temperature feedback to the temperature controller is obtained from a thermocouple (Type-T-08500-96, Cole-Parmer, Canada) inside the air bath. Two fans are used to circulate the air inside the air bath. The air bath keeps the desired constant temperature $\left(T=23.9 \pm 0.5^{\circ} \mathrm{C}\right)$. The actual temperature in the air bath is also measured and verified by using a thermometer.

A state-of-the-art experimental setup used for applying the above-described pressure decay method is established at the Petroleum Technology Research Centre (PTRC), University of Regina. A digital photograph of this setup is shown in Fig. 4.3.

\subsubsection{Experimental procedure}

Prior to each experiment, the diffusion cell and all the connections are pressurized with nitrogen and tested for any leakage several times. The following experimental procedure is implemented for recording the pressure decay due to the molecular diffusion. First, the diffusion cell is cleaned and evacuated. The temperature controller, fans and electric heater are turned on for the temperature maintenance. Then the diffusion cell is purged three times with the solvent to be tested and completely filled with it to reach certain pressure, which is estimated from the required initial pressure in the experiment and the volume of the heavy oil sample to be injected. After the diffusion cell is filled with the solvent, it is tested for leakage for 24 hours and its temperature is allowed to reach an equilibrium value. Finally, a pre-specified volume of heavy oil sample is injected from the heavy oil transfer cylinder into the diffusion cell at a constant flow rate by using the positive displacement pump. As the solvent gradually dissolves in heavy oil, the decaying pressure and constant temperature inside the diffusion cell are 


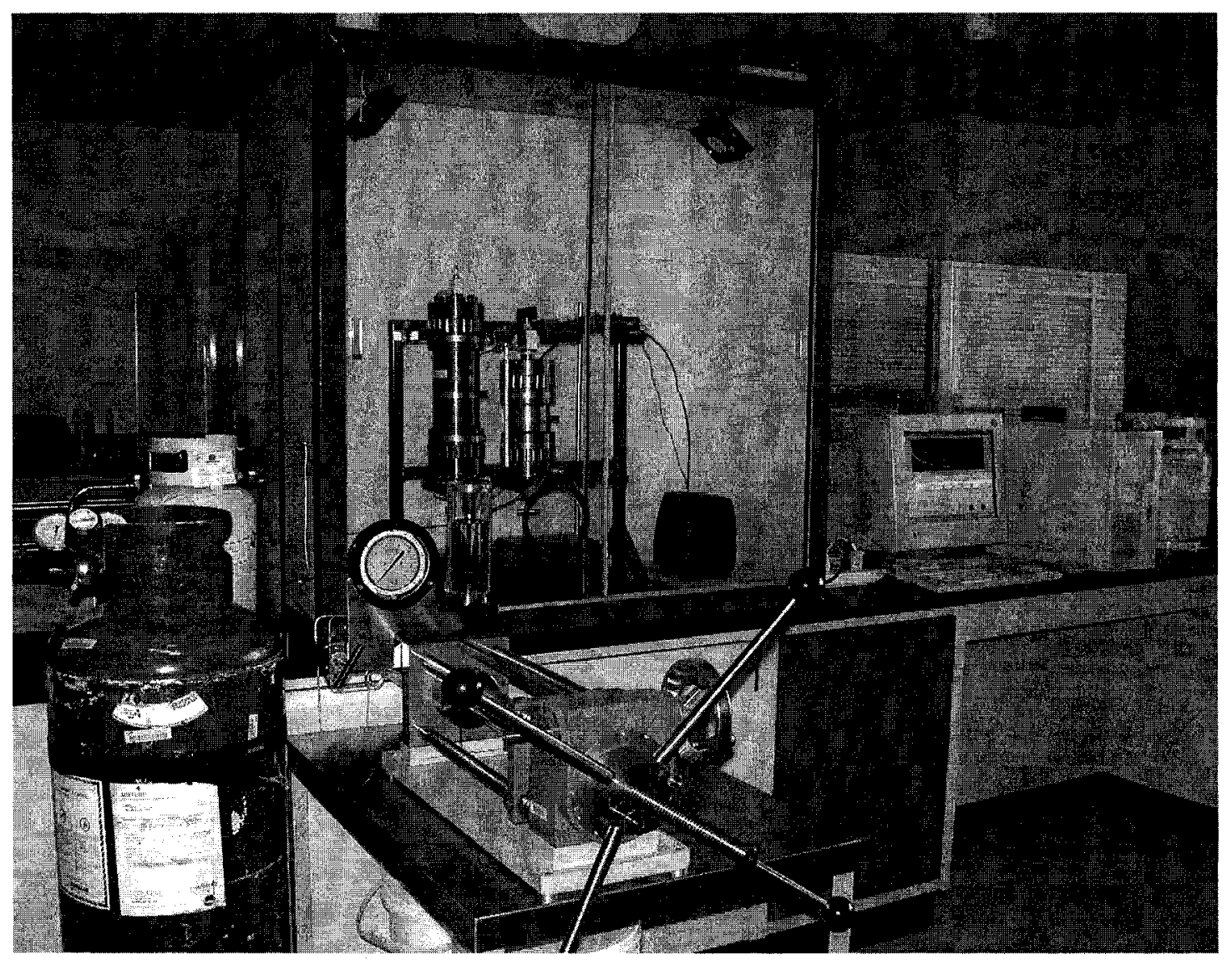

Figure 4.3 The experimental setup and the personal computer-based on-line data acquisition system for measuring the solvent-heavy oil diffusion coefficient by using the pressure decay method. 
acquired on-line, stored and displayed in the personal computer. These experimental data are recorded once every two minutes during each pressure decay measurement. The data acquisition process continues until the daily pressure decay is within the accuracy of the pressure transducer, i.e., approximately $3.0 \mathrm{kPa}$ per day.

\subsubsection{Measured pressure decay data}

In this thesis study, the pressure decay tests for three different solvents, $\mathrm{CO}_{2}, \mathrm{CH}_{4}$ and $\mathrm{C}_{3} \mathrm{H}_{8}$, are conducted at constant temperature of $T=23.9^{\circ} \mathrm{C}$. All the three experiments are repeated to verify and compare the obtained results. Therefore, a total of six runs are carried out in this thesis project, with two runs for each specific solvent-heavy oil system. A detailed summary of all the pressure decay experiments is given in Table 4.1. For each solvent-heavy oil system, the data presented in this table include the initial pressure, i.e., the pressure at the start of the experiment, the volume percentage of heavy oil in the diffusion cell, the termination time, i.e., time of experiment, and the termination pressure, i.e., the pressure at the time at which the experiment is terminated.

The obtained pressure decay data for a total of six runs conducted in this study are shown in Figs. 4.4 through 4.6. Specifically, Figures $4.4 \mathrm{a}$ and $4.4 \mathrm{~b}$ are for $\mathrm{CO}_{2}$-heavy oil system, Figures $4.5 \mathrm{a}$ and $4.5 \mathrm{~b}$ are for $\mathrm{CH}_{4}$-heavy oil system and Figures $4.6 \mathrm{a}$ and $4.6 \mathrm{~b}$ are for $\mathrm{C}_{3} \mathrm{H}_{8}$-heavy oil system. All these figures are plotted by using the measured data for every 30 minutes. Furthermore, these figures indicate that the pressure decays faster at the beginning and finally tends to approach an asymptotic value, i.e., the equilibrium pressure. The discontinuities in the measured pressure decay data for each solvent-heavy oil system are caused by neglecting some pressure data when the room temperature is above $23.9^{\circ} \mathrm{C}$. This is because the air bath used in this study can only maintain the system 
Table 4.1 Experimental conditions of the pressure decay measurements at $T=23.9^{\circ} \mathrm{C}$.

\begin{tabular}{|c|c|c|c|c|c|c|}
\hline \multirow{3}{*}{ Experimental parameters } & \multicolumn{6}{|c|}{ Solvent-heavy oil system } \\
\hline & \multicolumn{2}{|c|}{$\mathrm{CO}_{2}$-heavy oil } & \multicolumn{2}{|c|}{$\mathrm{CH}_{4}$-heavy oil } & \multicolumn{2}{|c|}{$\mathrm{C}_{3} \mathrm{H}_{8}$-heavy oil } \\
\hline & Run-1 & Run-2 & Run-1 & Run-2 & Run-1 & Run-2 \\
\hline Initial pressure $[\mathrm{kPa}]$ & $4,172.5$ & $4,210.0$ & $5,031.3$ & $5,004.2$ & 765.9 & 790.4 \\
\hline Volume percentage of heavy oil [v/v\%] & 27.2 & 25.5 & 16.4 & 24.4 & 27.4 & 25.9 \\
\hline Termination time [day] & 37.3 & 10.5 & 23.8 & 26.3 & 23.5 & 18.0 \\
\hline Termination pressure $[\mathrm{kPa}]$ & $3,530.0$ & $3,815.0$ & $4,918.0$ & $4,872.0$ & 393.3 & 482.0 \\
\hline
\end{tabular}




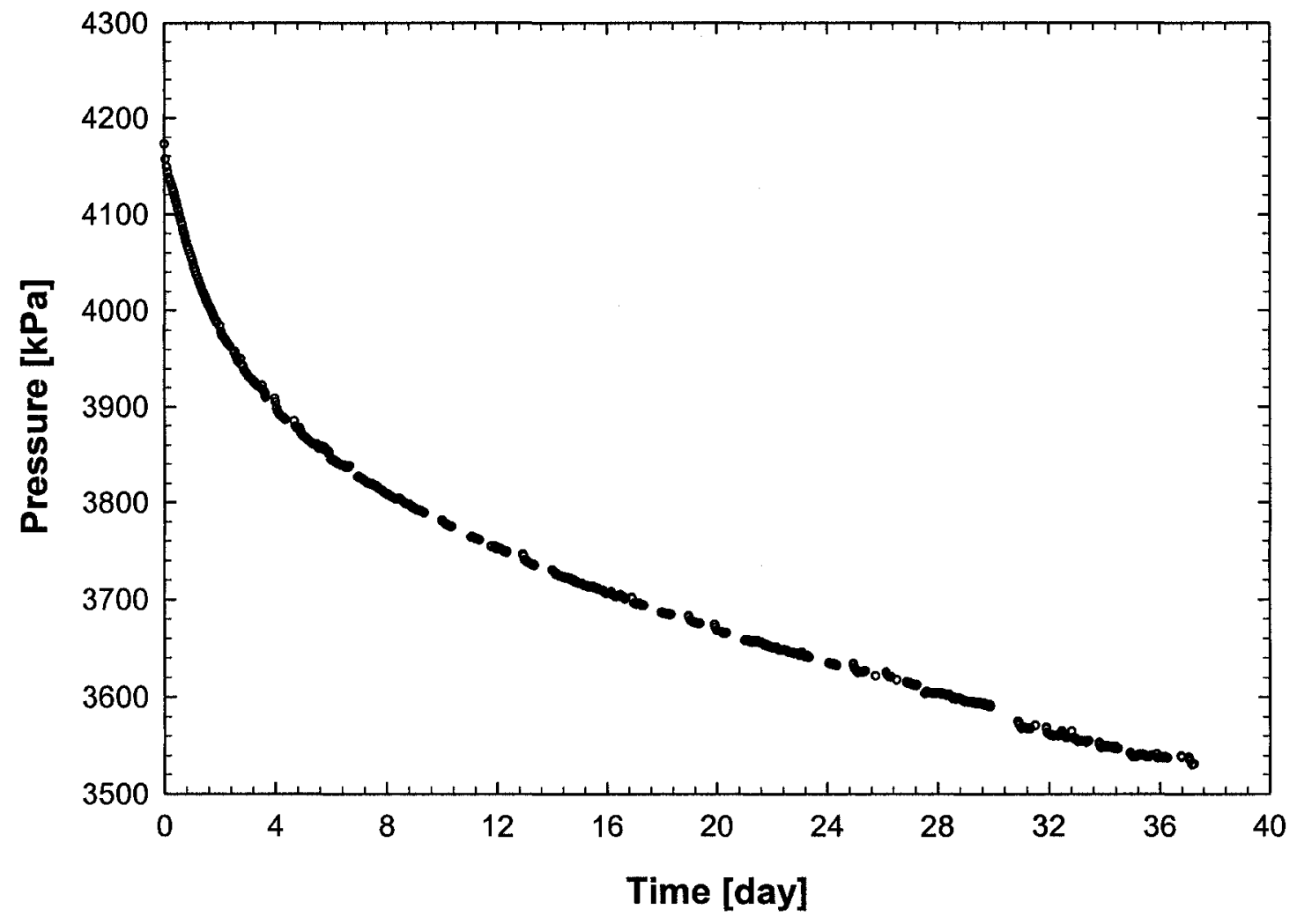

Figure 4.4a Measured pressure decay data for $\mathrm{CO}_{2}$-heavy oil system (Run-1) at $T=23.9^{\circ} \mathrm{C}$. 


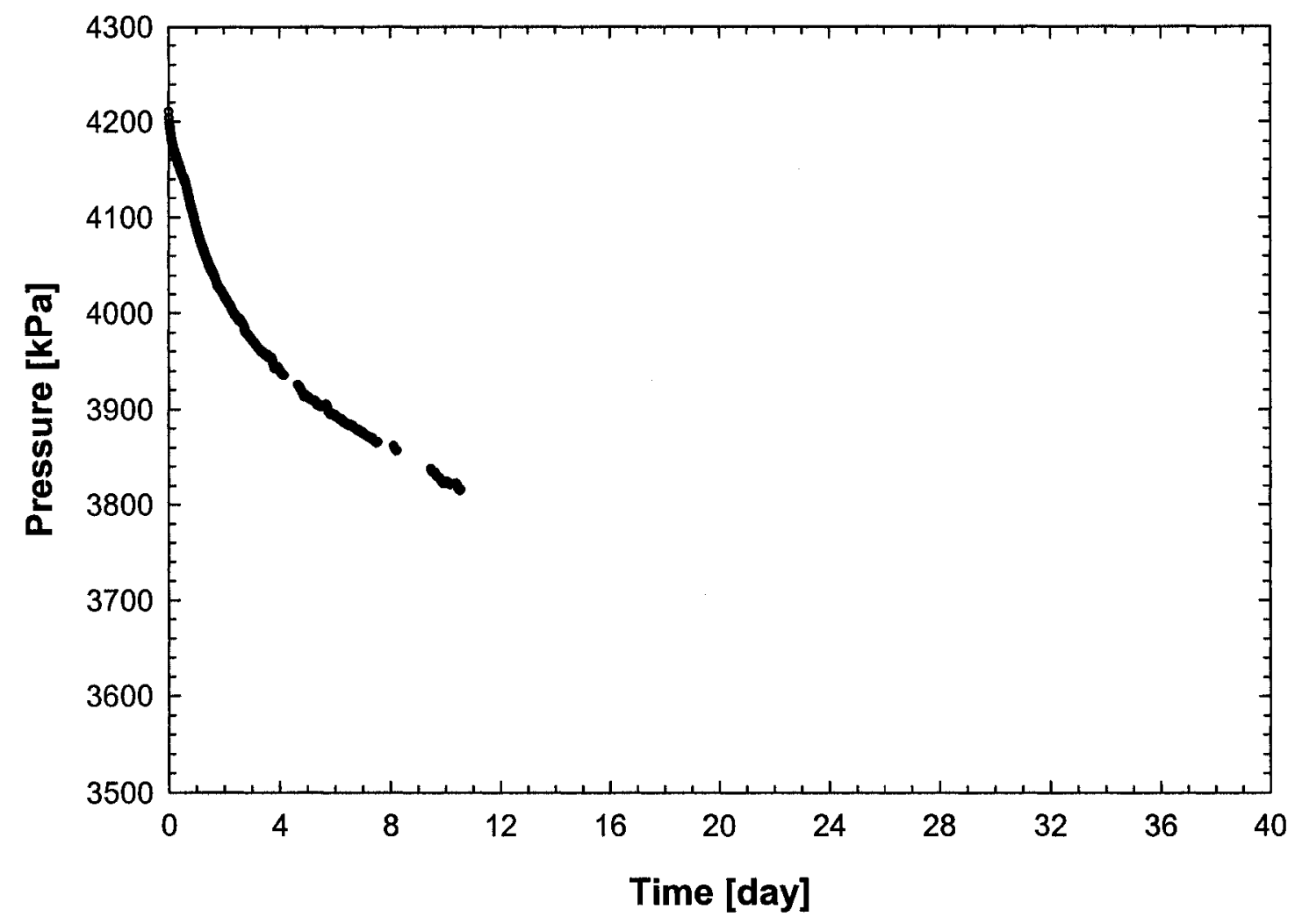

Figure 4.5b Measured pressure decay data for $\mathrm{CO}_{2}$-heavy oil system (Run-2) at $T=23.9^{\circ} \mathrm{C}$. 


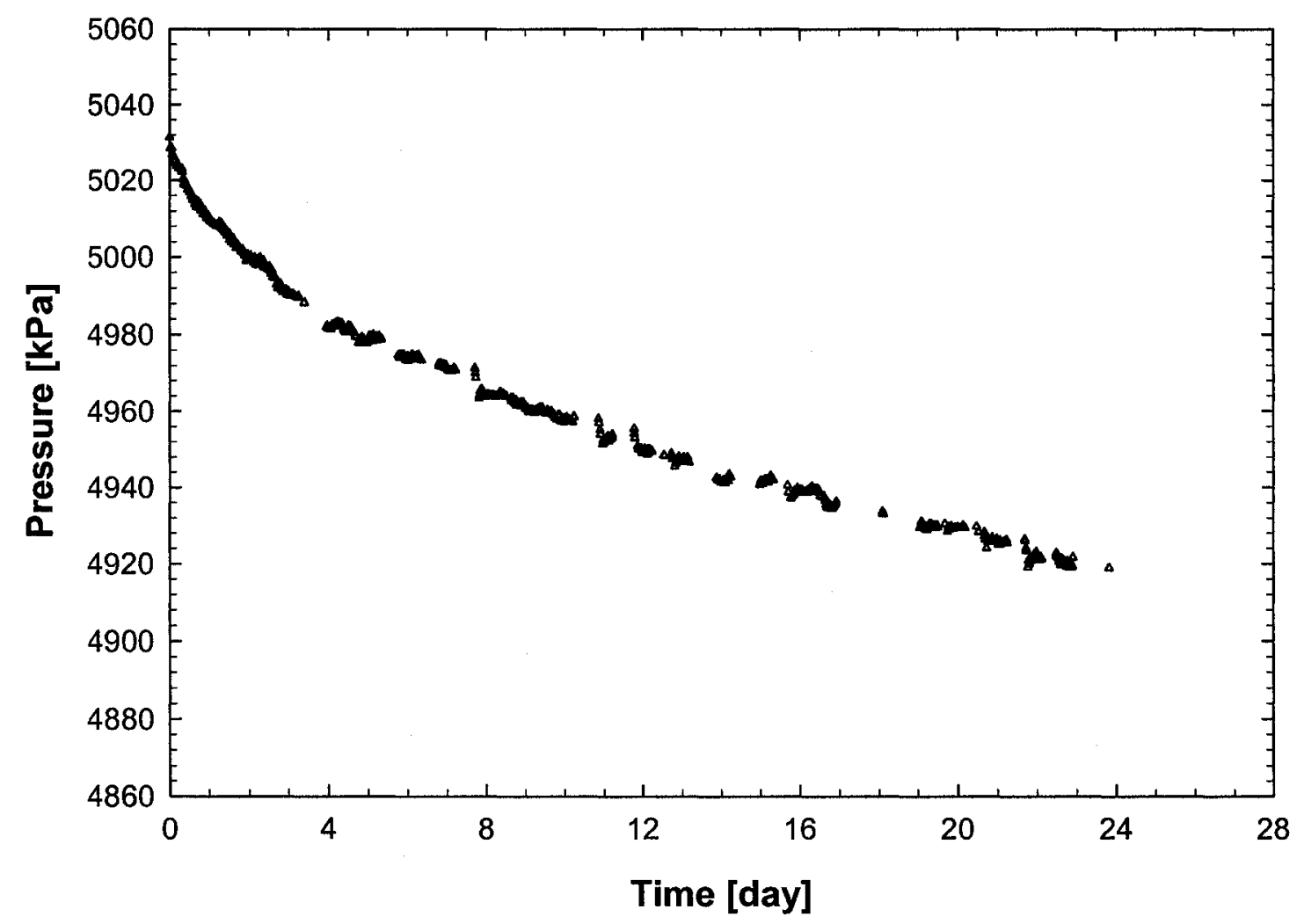

Figure 4.5a Measured pressure decay data for $\mathrm{CH}_{4}$-heavy oil system (Run-1) at $T=23.9^{\circ} \mathrm{C}$. 


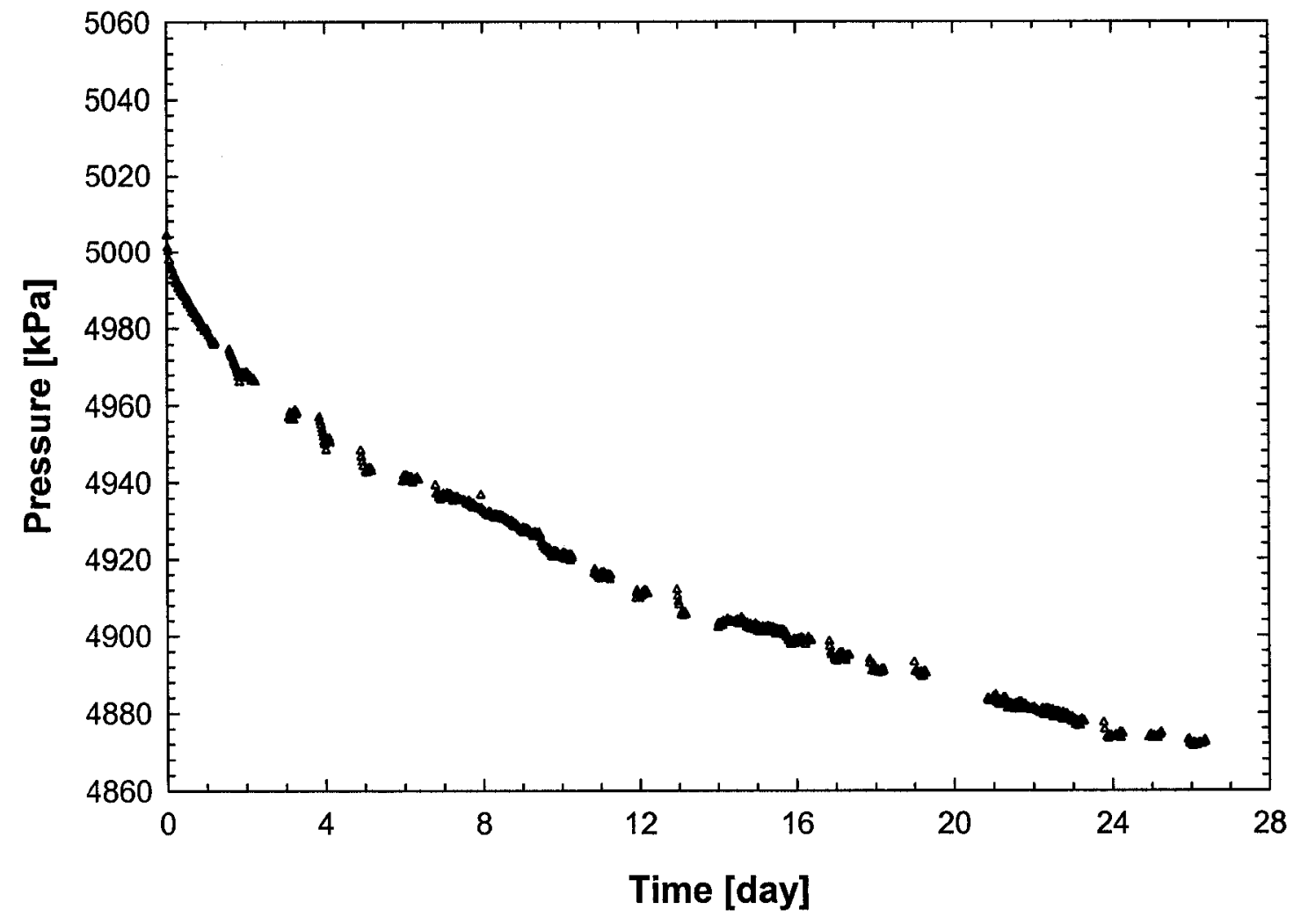

Figure 4.5b Measured pressure decay data for $\mathrm{CH}_{4}$-heavy oil system (Run-2) at $T=23.9^{\circ} \mathrm{C}$. 


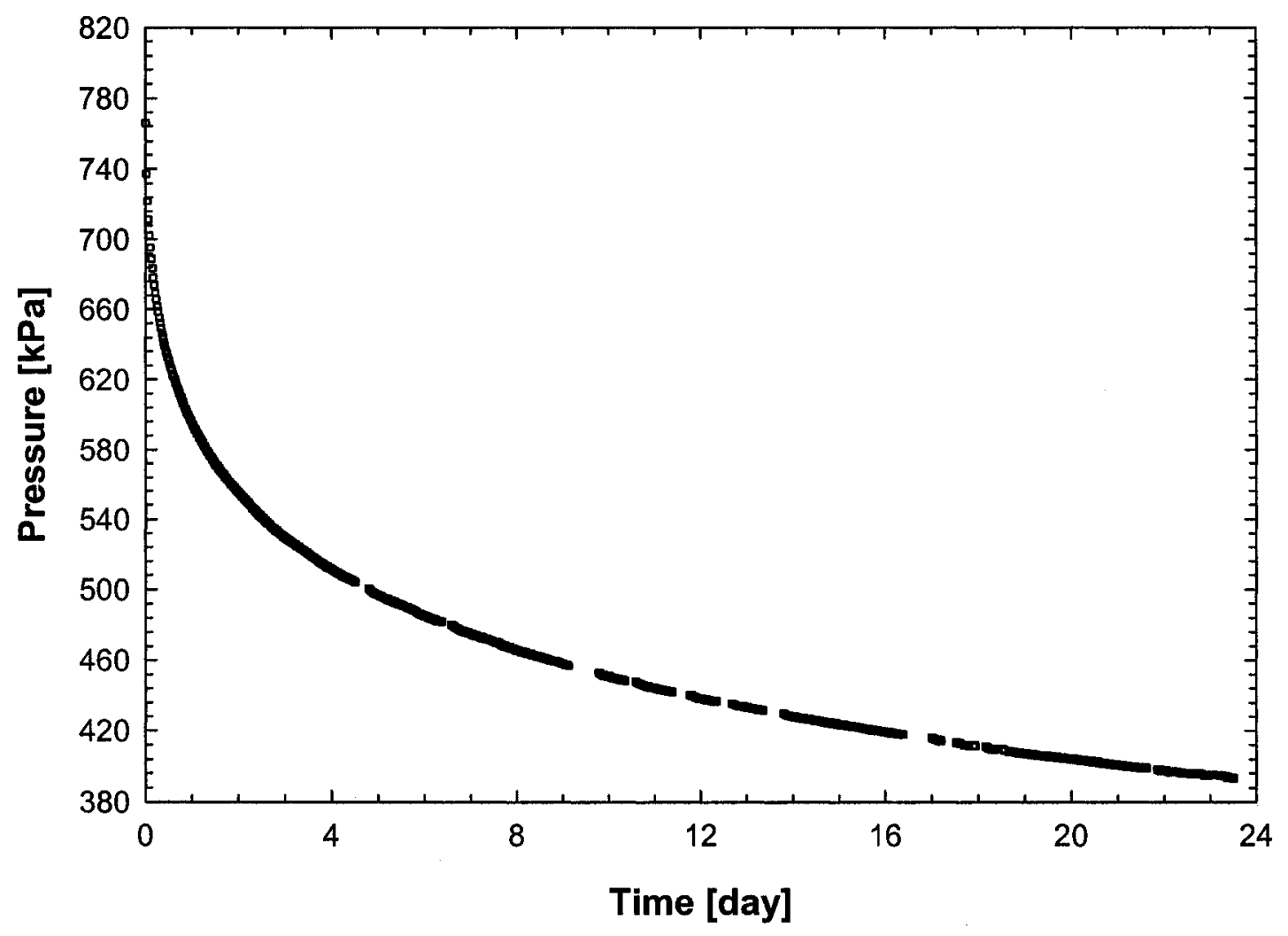

Figure 4.6a Measured pressure decay data for $\mathrm{C}_{3} \mathrm{H}_{8}$-heavy oil system (Run-1) at $T=23.9^{\circ} \mathrm{C}$. 


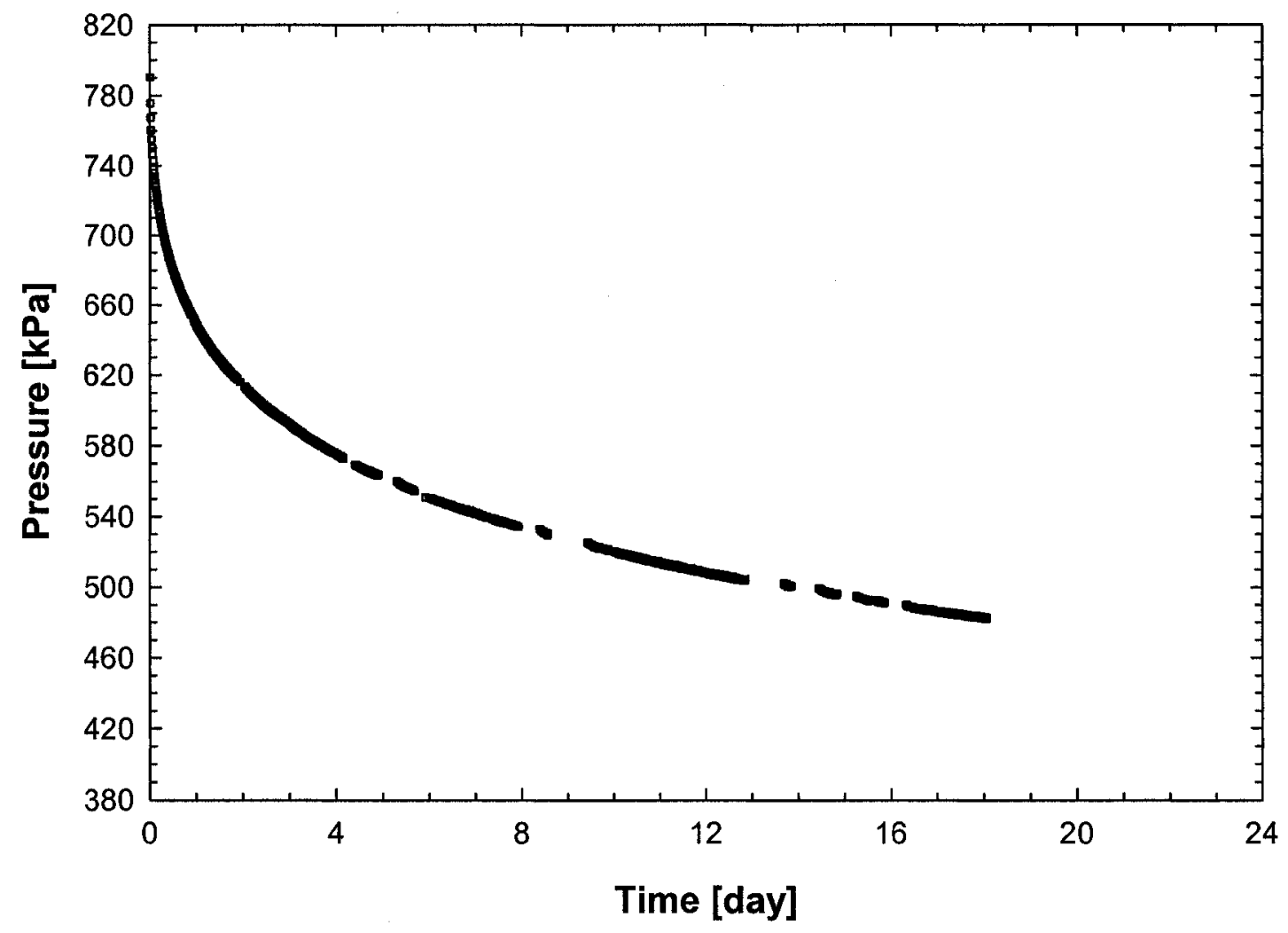

Figure 4.6b Measured pressure decay data for $\mathrm{C}_{3} \mathrm{H}_{8}$-heavy oil system (Run-2) at $T=23.9^{\circ} \mathrm{C}$. 
temperature above the room temperature by electrical heating. In addition, during the experiment, the pressure decay data for each solvent-heavy oil system are measured for a sufficiently long period, with an expectation for the system to reach the final equilibrium pressure. Nevertheless, the equilibrium pressure is still not reached during the experiment, as the diffusion process under investigation is extremely slow. Therefore, in this thesis study, a new approach is adopted to determine the equilibrium pressure at the same temperature from the measured solubility data for the same solvent-heavy oil system. The theoretical aspects of this approach have been thoroughly described in the previous chapter. The detailed experimental apparatus and the procedure for conducting the solubility measurements will be described in the subsequent section.

After the completion of each test, gas chromatographic analysis of the remaining solvent phase in the diffusion cell is undertaken to determine whether there are any hydrocarbon gases that are extracted from the heavy oil sample. It has been found that there are no hydrocarbon gases (i.e., no light-ends extraction) in $\mathrm{CO}_{2}$-heavy oil system and that there are no other hydrocarbon gases found in $\mathrm{CH}_{4}$-heavy oil system and in $\mathrm{C}_{3} \mathrm{H}_{8}$-heavy oil system, respectively. Thus, these findings support the assumption that there is only one-way diffusion from pure solvent phase to heavy oil, i.e., the heavy oil is non-volatile.

\subsection{Solubility Measurements}

\subsubsection{Experimental apparatus}

Figure 4.7 shows a schematic of the vapor-liquid equilibrium (VLE) apparatus for measuring the solubility of a solvent in heavy oil. The apparatus mainly consists of a see- 


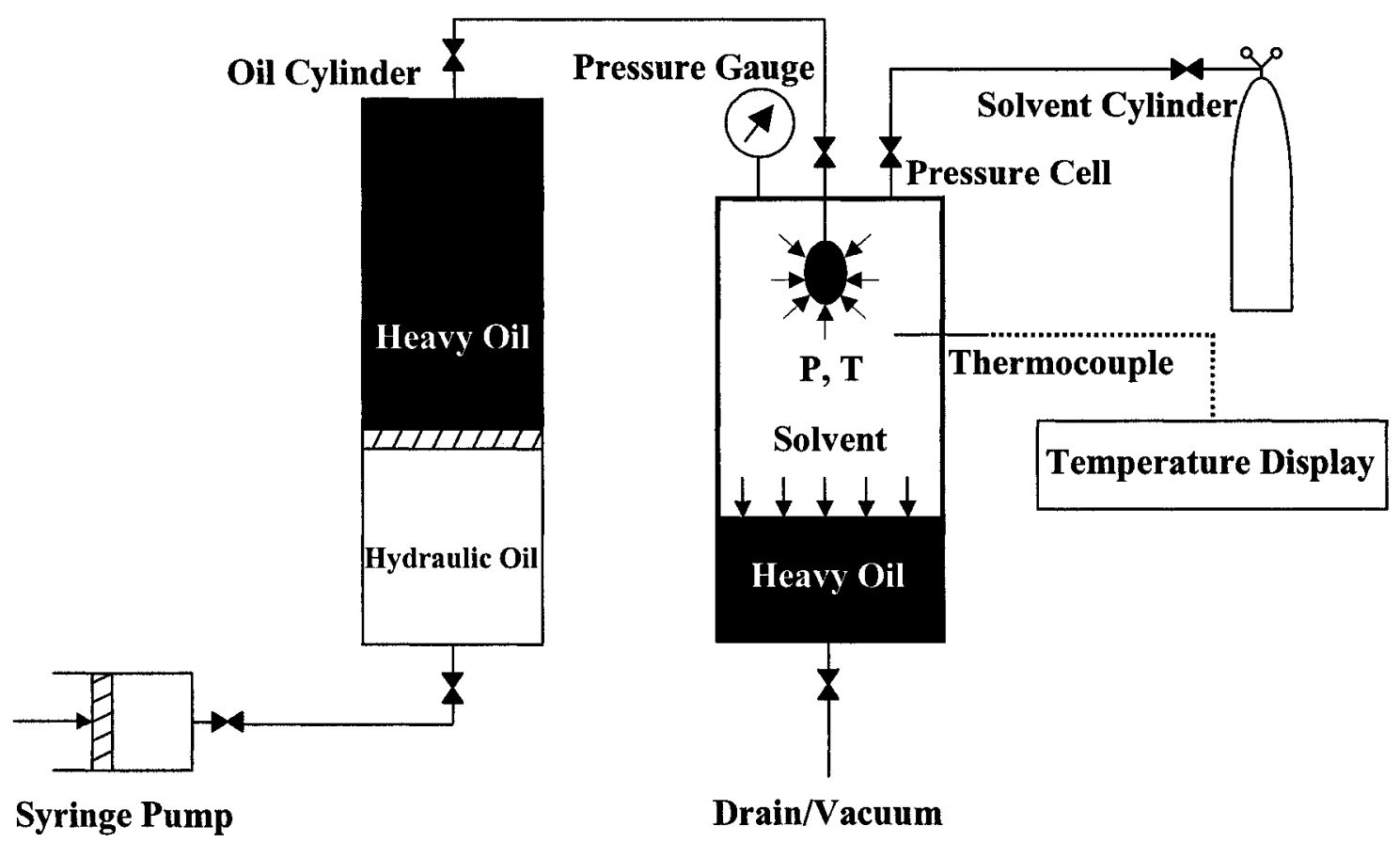

Figure 4.7 Schematic of the vapor-liquid equilibrium (VLE) apparatus used to measure the solubility of a solvent in heavy oil. 
through windowed high-pressure cell (P/N 2329-800, Ruska Fluid Products, Chandler Engineering, USA) and a programmable syringe pump (100DX, ISCO, Inc., USA). The see-through windowed pressure cell has an internal volume of $100 \mathrm{~cm}^{3}$ and can sustain pressure up to $69,000 \mathrm{kPa}$. The operating temperature of the pressure cell is from -17 to $148^{\circ} \mathrm{C}$. The programmable syringe pump can operate continuously and automatically to deliver accurate amount of heavy oil under a high pressure. Its flow rate ranges from $1 \times 10^{-5}$ to $50 \mathrm{~cm}^{3} / \mathrm{min}$ with an accuracy of $0.3 \%$. The displacement resolution is $9.65 \times 10^{-6}$ $\mathrm{cm}^{3}$. The temperature during the test is maintained at $T=23.9^{\circ} \mathrm{C}$ by wrapping the pressure cell with two heating tapes (HT95504×1, Electrothermal, USA), which are connected to a stepless temperature controller (CN45515, Thermolyne, USA). The pressure measurement is carried out by using a digital pressure gauge (DTG-6000, 3D Instruments, USA), which is connected to one of the top ports of the pressure cell. The full scale of the digital pressure gauge is $34,487 \mathrm{kPa}$. Its accuracy is equal to $0.02 \%$ of the full scale if the measured pressure is within 0 to $20 \%$ of the full scale, and $0.1 \%$ of the measured pressure in the range of 20 to $100 \%$ of the full scale, respectively. The abovedescribed vapor-liquid equilibrium (VLE) apparatus is constructed at the Petroleum Technology Research Centre (PTRC), University of Regina, for measuring the solubility of a gas solvent in heavy oil at different pressures and constant temperature. A digital photograph of the VLE apparatus is shown in Fig. 4.8.

\subsubsection{Experimental procedure}

Prior to each experiment, the pressure cell and all the connections are leak-tested with nitrogen. The pressure cell is then purged with the test solvent and filled with it to reach a pre-specified pressure. The initial cell pressure $P_{\mathrm{i}}$ and the solvent volume $V_{\mathrm{i}}$ are 


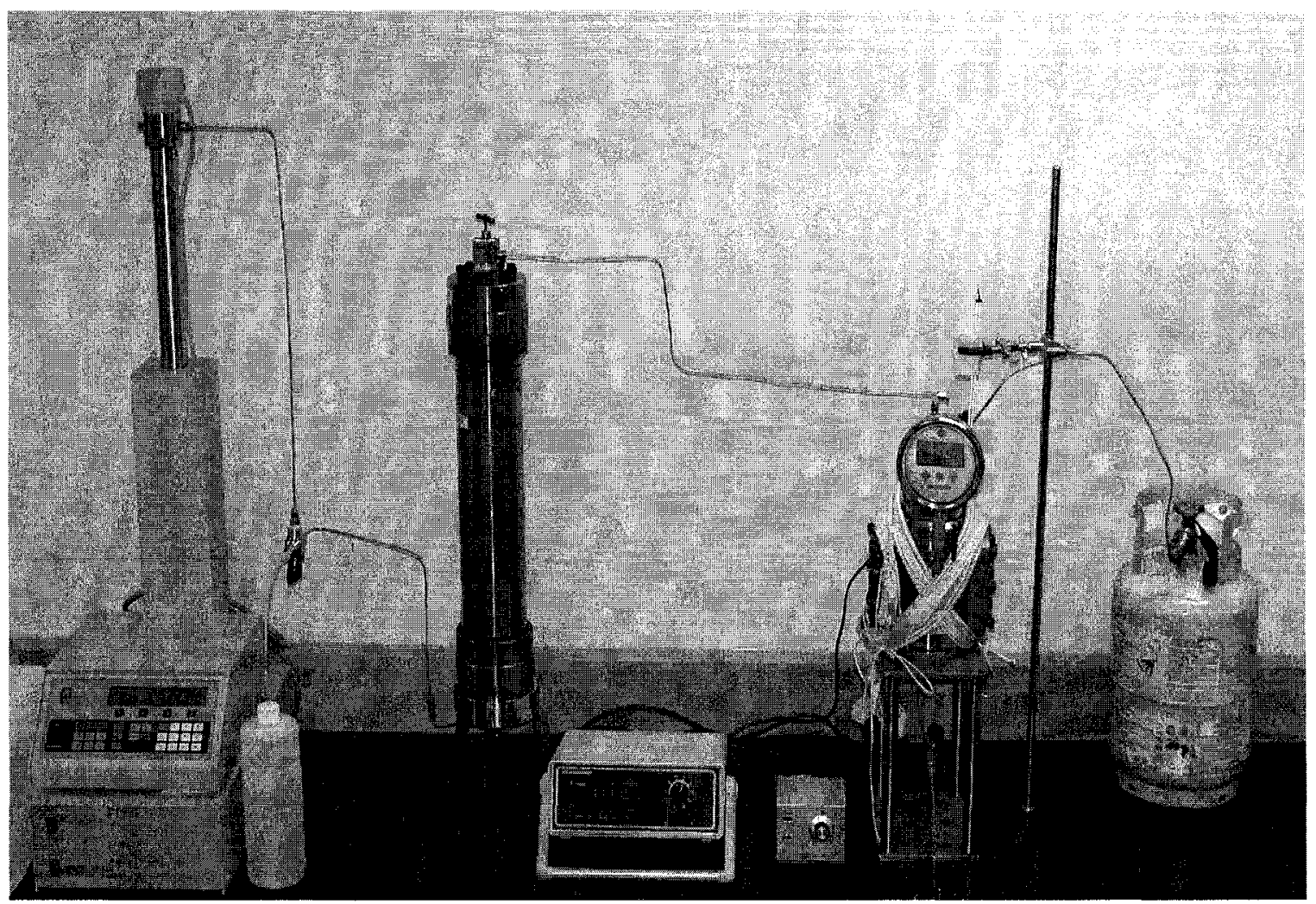

Figure 4.8 Vapor-liquid equilibrium (VLE) apparatus used to measure the solubility of a solvent in heavy oil. 
recorded before heavy oil sample is introduced. Then the heavy oil sample is injected slowly from the top port into the pressure cell by using the syringe pump at a constant flow rate of $0.005 \mathrm{~cm}^{3} / \mathrm{min}$. The slow injection process of heavy oil takes place by forming pendant oil drop continuously at the tip of $1 / 8^{\prime \prime}$ stainless steel tubing inside the pressure cell. Formation of the pendant oil drops at such a slow rate accelerates the mass transfer of the solvent into heavy oil by creating large contact area. As a result, rocking, circulating and stirring, which are frequently involved in the conventional methods (Frauenfeld et al., 2002) to accelerate the mass transfer process, are completely eliminated in the solubility measurements. After the required amount of heavy oil is injected into the pressure cell, the syringe pump is stopped and the pressure inside the pressure cell is recorded and monitored continuously. Eventually, the heavy oil is completely saturated and no pressure change is observed after 2-3 days. The corresponding pressure in the solvent phase, $P_{\mathrm{f}}$, is noted.

\subsubsection{Determination of solubility}

The following steps are involved in determining the solubility of each solvent in heavy oil. First, the initial mass of solvent is calculated by using the EOS for a real gas with the measured initial cell pressure, $P_{\mathrm{i}}$, and the initial solvent volume, $V_{\mathrm{i}}$. Then the mass of solvent remaining in the solvent phase under the final saturation condition is found from the EOS with the final saturation pressure, $P_{\mathrm{f}}$, and the final solvent volume, $V_{\mathrm{f}}$. Finally, the conservation law of solvent mass for the closed system is applied to compute the solubility of the solvent dissolved in heavy oil, $\chi$, which can be written as:

$$
\chi=\frac{M_{\text {solvent }}}{R T m_{\text {oil }}}\left[\frac{P_{\mathrm{i}} V_{\mathrm{i}}}{Z_{\mathrm{i}}\left(P_{\mathrm{i}}, T\right)}-\frac{P_{\mathrm{f}} V_{\mathrm{f}}}{Z_{\mathrm{f}}\left(P_{\mathrm{f}}, T\right)}\right] \times 100,
$$


where $Z_{\mathrm{i}}\left(P_{\mathrm{i}}, T\right)$ and $Z_{\mathrm{f}}\left(P_{\mathrm{f}}, T\right)$ are the $Z$-factors at the initial pressure $P_{\mathrm{i}}$ and the final saturation pressure $P_{\mathrm{f}}$ at the given temperature $T$, respectively. In this equation, the $\mathrm{Z}$ factors are also calculated by using the Lee-Kesler correlation (Lee and Kesler, 1975). The measured solvent solubility $\chi$ is expressed as the weight percentage of the mass of heavy oil sample at the corresponding final saturation pressure and given temperature.

\subsubsection{Measured solubility data}

The measured solubility data for $\mathrm{CO}_{2}$-heavy oil system, $\mathrm{CH}_{4}$-heavy oil system and $\mathrm{C}_{3} \mathrm{H}_{8}$-heavy oil system, are plotted in Fig. 4.9, Fig. 4.10 and Fig. 4.11, respectively. These solubility data are obtained by using the vapor-liquid equilibrium (VLE) apparatus. A linear regression of the measured solubility data is undertaken and the solubility, $\chi(P, T)$, is expressed as a linear function of pressure at $T=23.9^{\circ} \mathrm{C}$ as shown in Fig. 4.9 for $\mathrm{CO}_{2}$-heavy oil system, in Fig. 4.10 for $\mathrm{CH}_{4}$-heavy oil system, and in Fig. 4.11 for $\mathrm{C}_{3} \mathrm{H}_{8}$-heavy oil system, respectively. The correlation coefficients of the linear regression show excellent linearity between the measured solubility and the pressure for both carbon dioxide-heavy oil and methane-heavy oil systems in the pressure ranges tested. Furthermore, the correlation coefficient of the linear regression for propane-heavy oil system indicates good linearity between the measured solubility and the pressure in the pressure range tested. Such linearity is also reported for other $\mathrm{CO}_{2}$-heavy oil/bitumen systems (Mehrotra et al., 1985; 1992; Quail et al., 1988), $\mathrm{CH}_{4}$-heavy oil/bitumen systems (Svrcek and Mehrotra, 1982; Mehrotra and Svrcek, 1985) and $\mathrm{C}_{3} \mathrm{H}_{8}$-heavy oil/bitumen systems (Fraunfeld et al., 2002). It can also be noted that the pressure range chosen for measuring the solubility of propane-heavy oil system is about one order lower than those tested for the other two solvent-heavy oil systems. This is because the vapour pressure of 


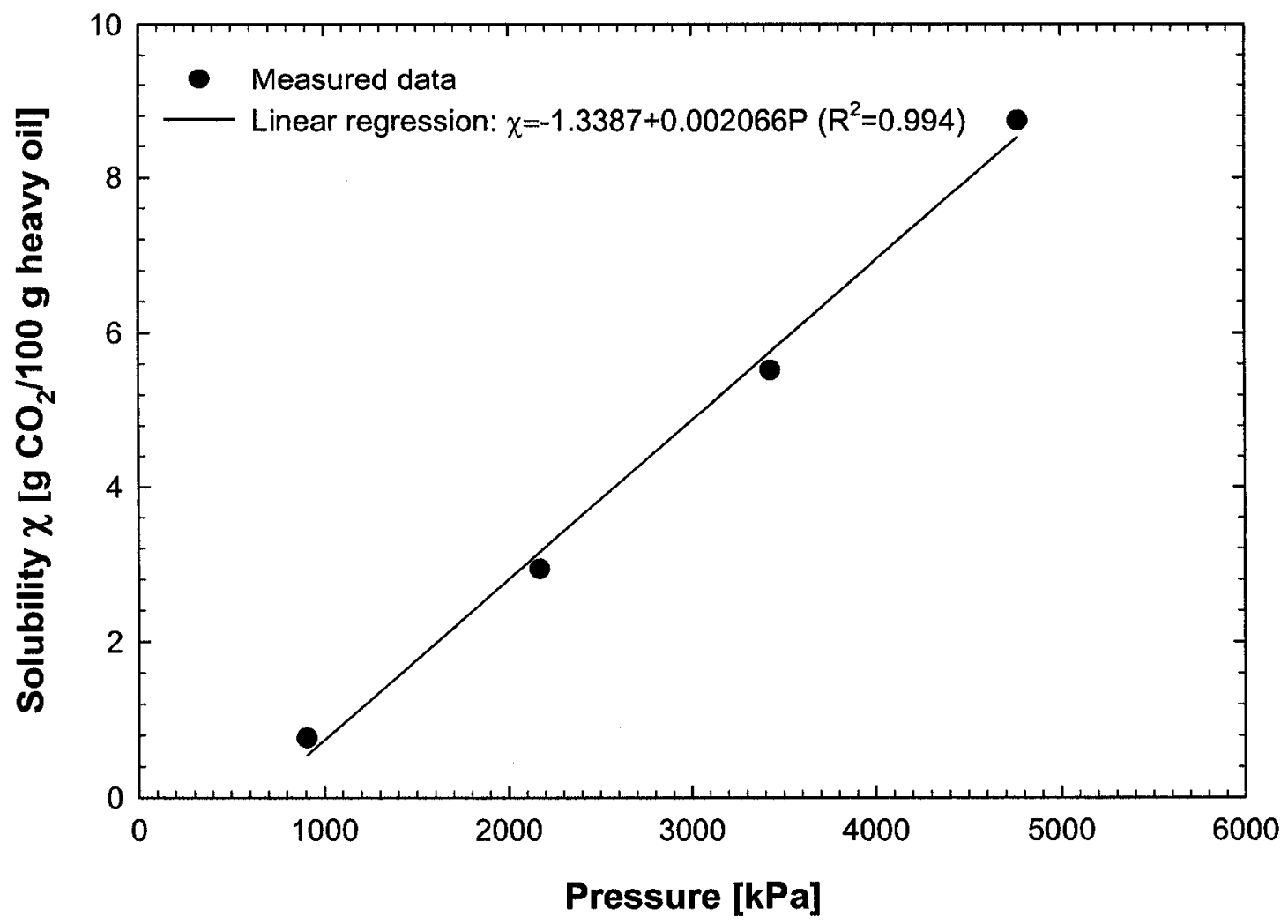

Figure 4.9 Measured solubility of $\mathrm{CO}_{2}$ in heavy oil as a function of the pressure at $T=23.9^{\circ} \mathrm{C}$. 


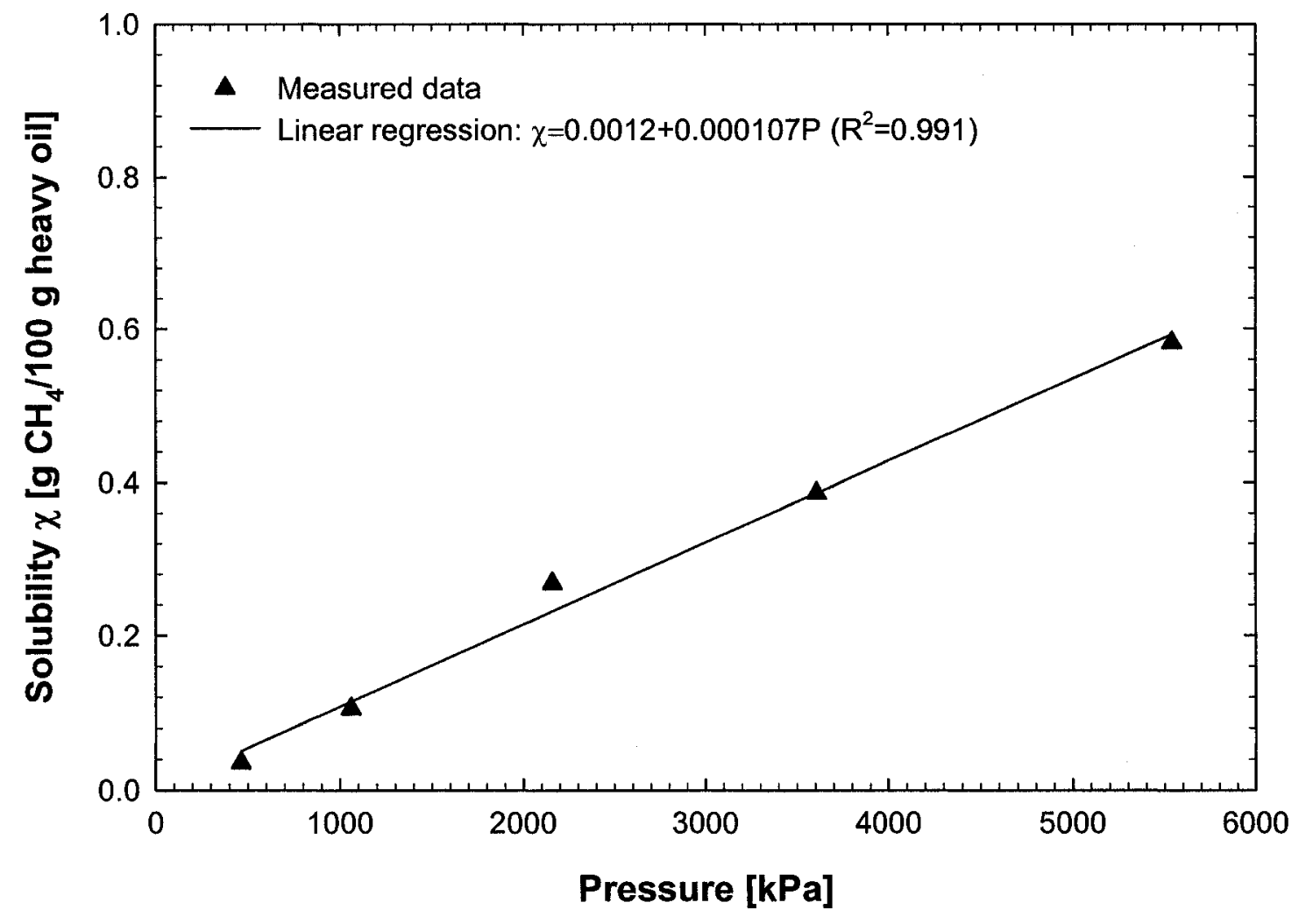

Figure 4.10 Measured solubility of $\mathrm{CH}_{4}$ in heavy oil as a function of the pressure at $T=23.9^{\circ} \mathrm{C}$. 


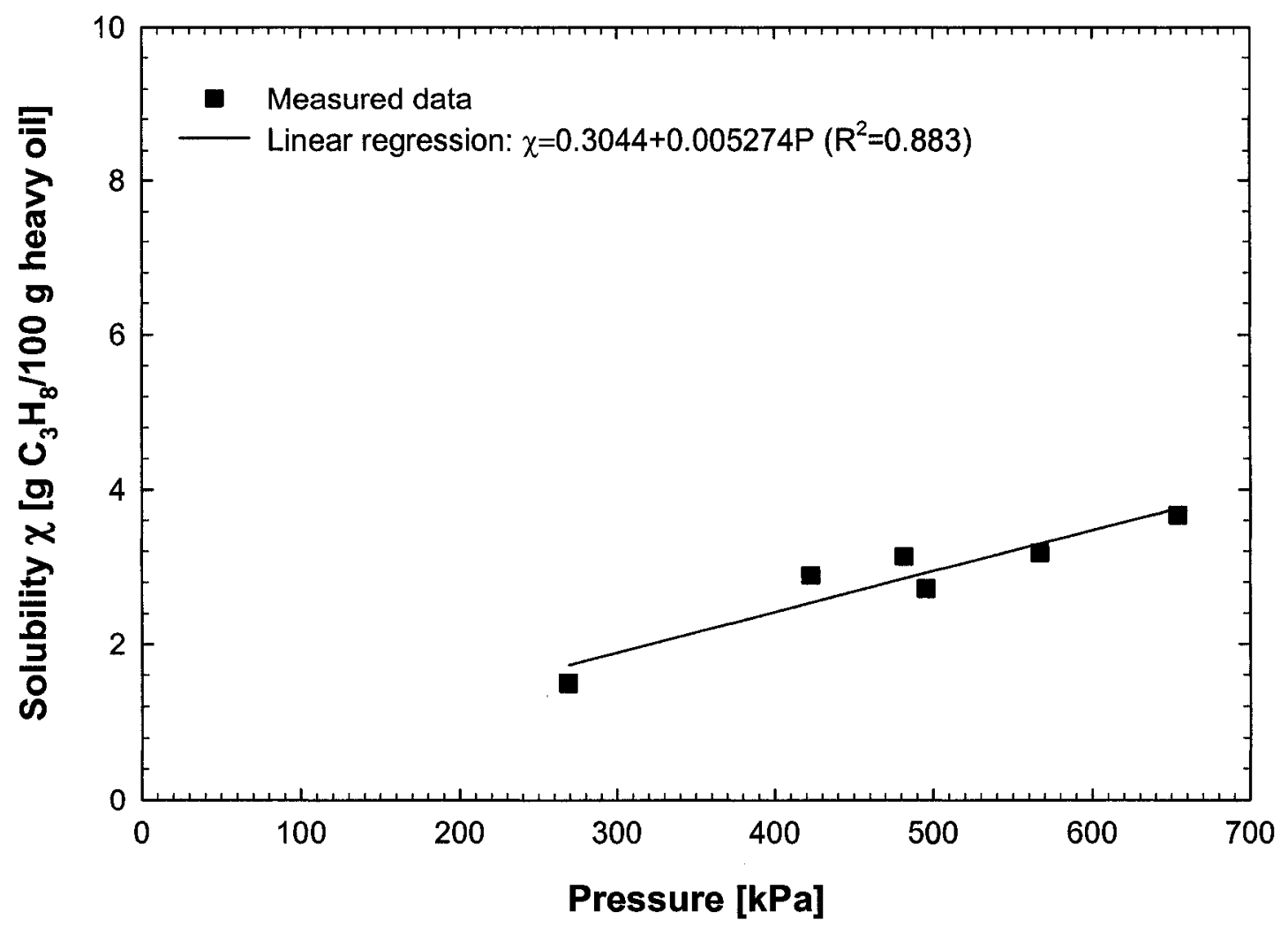

Figure 4.11 Measured solubility of $\mathrm{C}_{3} \mathrm{H}_{8}$ in heavy oil as a function of the pressure at $T=23.9^{\circ} \mathrm{C}$. 
propane is very low $(\sim 950 \mathrm{kPa})$, in comparison with carbon dioxide $(\sim 6,200 \mathrm{kPa})$ and methane $(\sim 31,700 \mathrm{kPa})$ at $23.9^{\circ} \mathrm{C}$ (Edmister and Lee, 1984; McCain, Jr., 1990). Obviously, the solvent becomes liquid when its pressure is above its vapour pressure. Accordingly, the solubility measurement cannot be conducted as the EOS cannot be applied in this case. Here, it is worthwhile to point out that the solubility data are required in order to determine the equilibrium pressure $P_{\text {eq }}$ of the pressure decay test for the respective solvent-heavy oil system.

In addition, comparison of the measured solubility data in Figs. 4.9 and 4.10 shows that the solubility of carbon dioxide in heavy oil is approximately one order higher than that of methane in heavy oil. Therefore, much more carbon dioxide can diffuse into heavy oil than methane under the same pressure and temperature conditions. On the other hand, it is also noted from Fig. 4.11 that the solubility of propane in the heavy oil sample is relatively high even at low pressures. In principle, solubility of a solvent in heavy oil can be related to its critical temperature and normal boiling point. It has been stated that a gas with a higher critical temperature and a higher normal boiling point is more soluble in hydrocarbon liquids (Nguyen and Farouq Ali, 1998). The critical temperatures of carbon dioxide, methane and propane are equal to $31.06,-82.50$ and $96.70^{\circ} \mathrm{C}$, respectively (Whitson and Brule, 2000). Their respective normal boiling points are $-78.20,-161.49$ and $-42.04^{\circ} \mathrm{C}$ (Whitson and Brule, 2000). Thus, propane has the highest solubility in heavy oil in comparison with carbon dioxide, which is followed by methane. 


\section{Chapter 5 RESULTS AND DISCUSSION}

This chapter consists of three major sections. In the first section, the three interface mass transfer models described in Chapter 3 are studied by using the previously published pressure decay data (Zhang et al., 2000) for carbon dioxide-heavy oil and methane-heavy oil systems. The second section presents the results obtained from the pressure decay experiments conducted in this thesis study to determine the diffusion coefficients of carbon dioxide, methane and propane in a given heavy oil sample. Finally, the third section gives a summary of the major results achieved in this thesis study.

\subsection{Interface Mass Transfer Studies Based on the Pressure Decay Data Available in the Literature}

\subsubsection{Pressure decay data}

In the literature, Zhang et al. (2000) carried out the pressure decay experiments for both the $\mathrm{CO}_{2}$-heavy oil and $\mathrm{CH}_{4}$-heavy oil systems to assess the feasibility of determining the diffusion coefficients by applying the pressure decay method. A constant volume stainless steel cylinder with the internal cross-sectional area of $4.94 \mathrm{~cm}^{2}$ and the height of $25.0 \mathrm{~cm}$ was used to perform the pressure decay experiments at the room temperature of $21^{\circ} \mathrm{C}$. These pressure decay experiments were conducted for 20 days in the case of $\mathrm{CO}_{2}-$ heavy oil system and 5 days for $\mathrm{CH}_{4}$-heavy oil system, respectively. The detailed experimental conditions for these two experiments are given in Table 5.1. The values of four parameters, $m_{1}, m_{2}, k_{1}$ and $k_{2}$, for each system are taken from the literature (Zhang et al., 2000) and also listed in this table. In principle, as described in Chapter 3, the pressure at the very end of the diffusion process (i.e., when the heavy oil is completely saturated 
Table 5.1 Experimental conditions of the pressure decay measurements conducted by Zhang et al. (2000) with the heavy oil of viscosity $\mu=5,000 \mathrm{mPa} \cdot \mathrm{s}$ at $T=21^{\circ} \mathrm{C}$ and four parameters required in Eq. [3.12].

\begin{tabular}{|c|r|r|}
\hline $\begin{array}{c}\text { Experimental conditions } \\
\text { and four parameters }\end{array}$ & $\mathrm{CO}_{2}$-heavy oil system & $\mathrm{CH}_{4}$-heavy oil system \\
\hline Initial pressure $[\mathrm{kPa}]$ & $3,427.0$ & $3,471.0$ \\
\hline Volume percentage of heavy oil [v/v\%] & 28.0 & 26.8 \\
\hline Termination pressure $[\mathrm{kPa}]$ & $2,856.0$ & $3,420.0$ \\
\hline Duration of experiment $[\mathrm{day}]$ & 20.0 & 5.0 \\
\hline$m_{1}[\mathrm{kPa}]$ & 575.9 & 23.97 \\
\hline$m_{2}[\mathrm{kPa}]$ & 11.07 & 35.88 \\
\hline$k_{1}[\mathrm{day}]$ & 4.83 & 2.44 \\
\hline$k_{2}[\mathrm{day}]$ & 4.73 & 0.1245 \\
\hline
\end{tabular}


with the solvent) is referred to as the equilibrium pressure. It should be noted that, however, Zhang et al. (2000) assumed the termination pressure of each of these experiments to be the equilibrium pressure, though their diffusion experiments did not reach the saturation state. The measured pressure decay data from these experiments are utilized in this study to examine the three interface mass transfer models and determine the diffusion coefficient of a solvent in heavy oil (Tharanivasan et al., 2003; 2004a).

\subsubsection{Carbon dioxide-heavy oil system}

By following the procedure described in Chapter 3, the diffusion coefficient of $\mathrm{CO}_{2}$-heavy oil system is determined by using the three developed interface mass transfer models. Table 5.2 compares the diffusivity data obtained in this study with the previously published data for the same system.

A plot of the average pressure difference $\Delta P_{\text {ave }}$ versus the diffusion coefficient $D$ of $\mathrm{CO}_{2}$-heavy oil system is shown in Fig. 5.1 for the equilibrium $\mathrm{BC}$ and the quasiequilibrium $\mathrm{BC}$, respectively. It is seen from Fig. 5.1 that the minimum $\Delta P_{\text {ave }}$ of $24.2 \mathrm{kPa}$ occurs at $D=4.1 \times 10^{-9} \mathrm{~m}^{2} / \mathrm{s}$ for the equilibrium $\mathrm{BC}$ and $28.8 \mathrm{kPa}$ at $D=3.4 \times 10^{-9} \mathrm{~m}^{2} / \mathrm{s}$ for the quasi-equilibrium $\mathrm{BC}$, respectively. If the minimum $\Delta P_{\text {ave }}$ values for these two $\mathrm{BCs}$ are compared, the equilibrium $\mathrm{BC}$ at the interface is better than the quasi-equilibrium $\mathrm{BC}$ to describe the mass transfer across $\mathrm{CO}_{2}$-heavy oil interface. Moreover, $D=4.1 \times 10^{-9} \mathrm{~m}^{2} / \mathrm{s}$ for the equilibrium $\mathrm{BC}$ obtained in this study is in good agreement with $D=4.8 \times 10^{-9}$ $\mathrm{m}^{2} / \mathrm{s}$ published by Zhang et al. (2000) for the same system.

Similar results for the non-equilibrium BC are plotted in Fig. 5.2 for several values of the mass-transfer Biot number $k_{\mathrm{D}}$. These results show that as long as $k_{\mathrm{D}}$ is equal to or less than 0.6 , the minimum $\Delta P_{\text {ave }}$ remains at about $10.7 \mathrm{kPa}$. Although the diffusion 
Table 5.2 Comparison of the results obtained in this study with the previously published data for $\mathrm{CO}_{2}$-heavy oil system (Zhang et al., 2000) at $T=21^{\circ} \mathrm{C}$.

\begin{tabular}{|l|l|c|c|}
\hline \multicolumn{1}{|c|}{ Reference } & Boundary condition & $\begin{array}{c}\text { Diffusion coefficient } \\
D\left[10^{-9} \mathrm{~m}^{2} / \mathrm{s}\right]\end{array}$ & $\begin{array}{c}\text { Biot number } \\
k_{\mathrm{D}}\end{array}$ \\
\hline \multirow{3}{*}{ This study } & Equilibrium & 4.1 & - \\
\cline { 2 - 4 } & Quasi-equilibrium & 3.4 & - \\
\cline { 2 - 4 } & Non-equilibrium & $>25.0$ & $<0.6$ \\
\hline Zhang et al. (2000) & Equilibrium & 4.8 & 14.7 \\
\hline Civan and Rasmussen (2002) & Non-equilibrium & 0.084 & 144.49 \\
\hline Civan and Rasmussen (2003) & Non-equilibrium & 4.9 & - \\
\hline
\end{tabular}




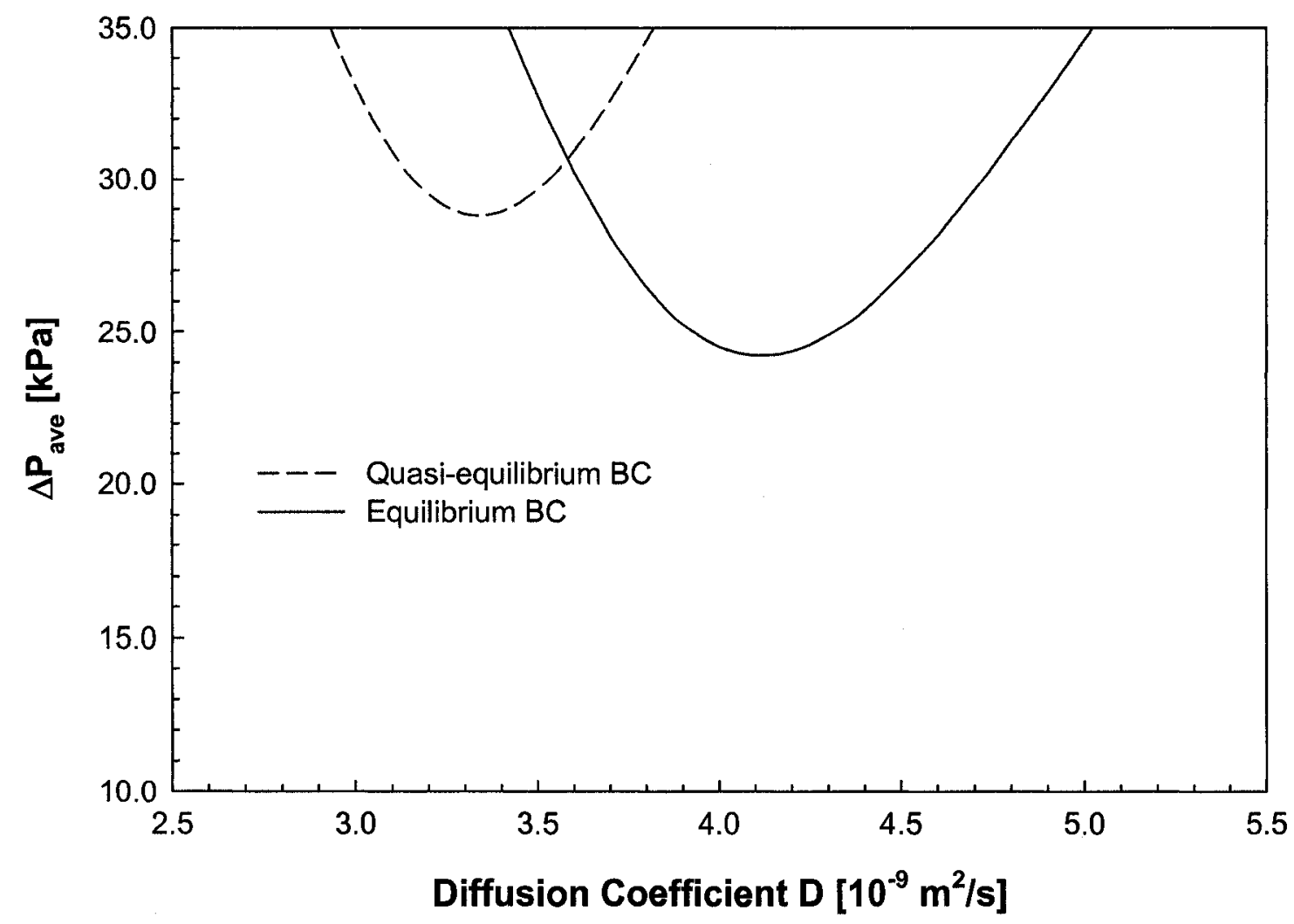

Figure 5.1 Variations of the average pressure difference $\Delta P_{\text {ave }}$ with the diffusion coefficient $D$ under the equilibrium and quasi-equilibrium boundary conditions for $\mathrm{CO}_{2}$ heavy oil system, based on the pressure decay data published by Zhang et al. (2000). 


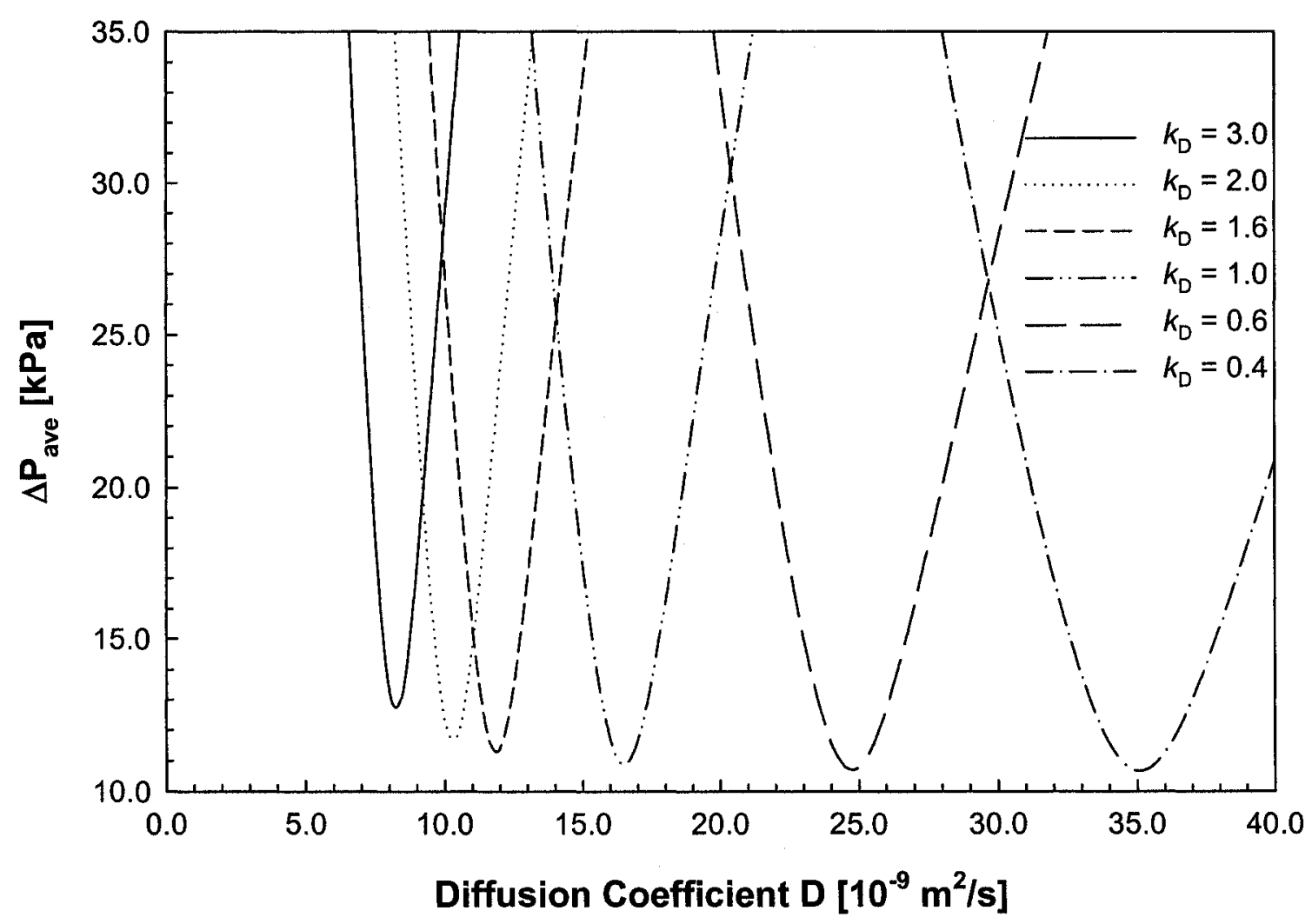

Figure 5.2 Variations of the average pressure difference $\Delta P_{\text {ave }}$ with the diffusion coefficient $D$ under the non-equilibrium boundary condition for $\mathrm{CO}_{2}$-heavy oil system, based on the pressure decay data published by Zhang et al. (2000). 
coefficient $D$ and the mass-transfer Biot number $k_{\mathrm{D}}$ cannot be uniquely determined for this $\mathrm{BC}$, the minimum $\Delta P_{\mathrm{ave}}$ values obtained in this case are much smaller than those for the other two $\mathrm{BCs}$. This fact indicates that the non-equilibrium $\mathrm{BC}$ at $\mathrm{CO}_{2}$-heavy oil interface is more applicable to this system in comparison with the equilibrium $\mathrm{BC}$ and the quasi-equilibrium BC. Physically, the non-equilibrium BC approaches the equilibrium $\mathrm{BC}$ when $k_{\mathrm{D}}$ is sufficiently large, noting that $k_{\mathrm{D}}$ represents the ratio of the bulk resistance $L / D$ for the molecular diffusion to the interfacial resistance $1 / k$ for the mass transfer.

It should also be noted that, as shown in Fig 5.2, the minimum objective functions are small for small values of $k_{\mathrm{D}}$ but become insensitive to the mass-transfer Biot number $k_{\mathrm{D}}$. Thus neither the global optimal $D$ nor the optimal $k_{\mathrm{D}}$ can be accurately determined. In this case, the determination of diffusion coefficient is carried out by assuming $k_{\mathrm{D}}=0$. With this assumption, the solvent concentration distribution in the bulk heavy oil phase is uniform. Hence, the uniform concentration, $c_{\mathrm{u}}$, for $k_{\mathrm{D}}=0$ is found from Eq. [3.17]. The average pressure difference in this case is minimized by using $k$ as an adjustable parameter and following the same procedure as described in Chapter 3 for the oneparameter simultaneous search method. Figure 5.3 shows that the minimum $\Delta P_{\text {ave }}$ of 10.7 $\mathrm{kPa}$ occurs when the interface mass transfer coefficient $k$ is equal to $1.77 \times 10^{-7} \mathrm{~m} / \mathrm{s}$. It is worthwhile to note that the diffusion coefficient cannot be determined in this case. This fact also implies that a sufficient amount of heavy oil sample should be used in the pressure decay measurement in order to have a reasonable value of $k_{\mathrm{D}}$.

The detailed results of the history matching of the calculated pressures with the measured pressures for all the three BCs are shown in Fig. 5.4. This figure shows that, for the equilibrium and quasi-equilibrium BCs, the differences between the calculated and 


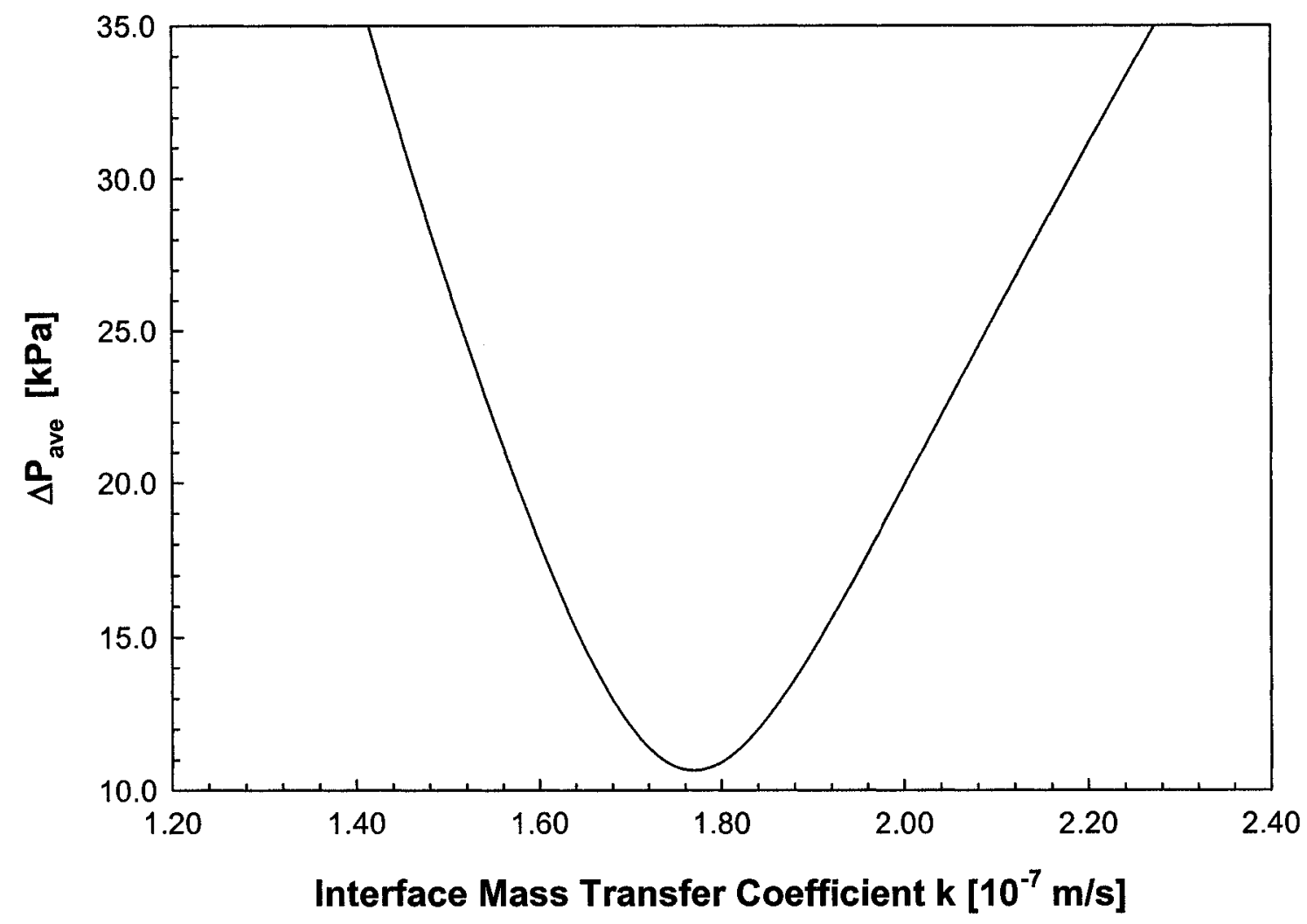

Figure 5.3 Variations of the average pressure difference $\Delta P_{\text {ave }}$ with the interface mass transfer coefficient $k$ in the case of $k_{\mathrm{D}}=0$ for $\mathrm{CO}_{2}$-heavy oil system, based on the pressure decay data published by Zhang et al. (2000). 


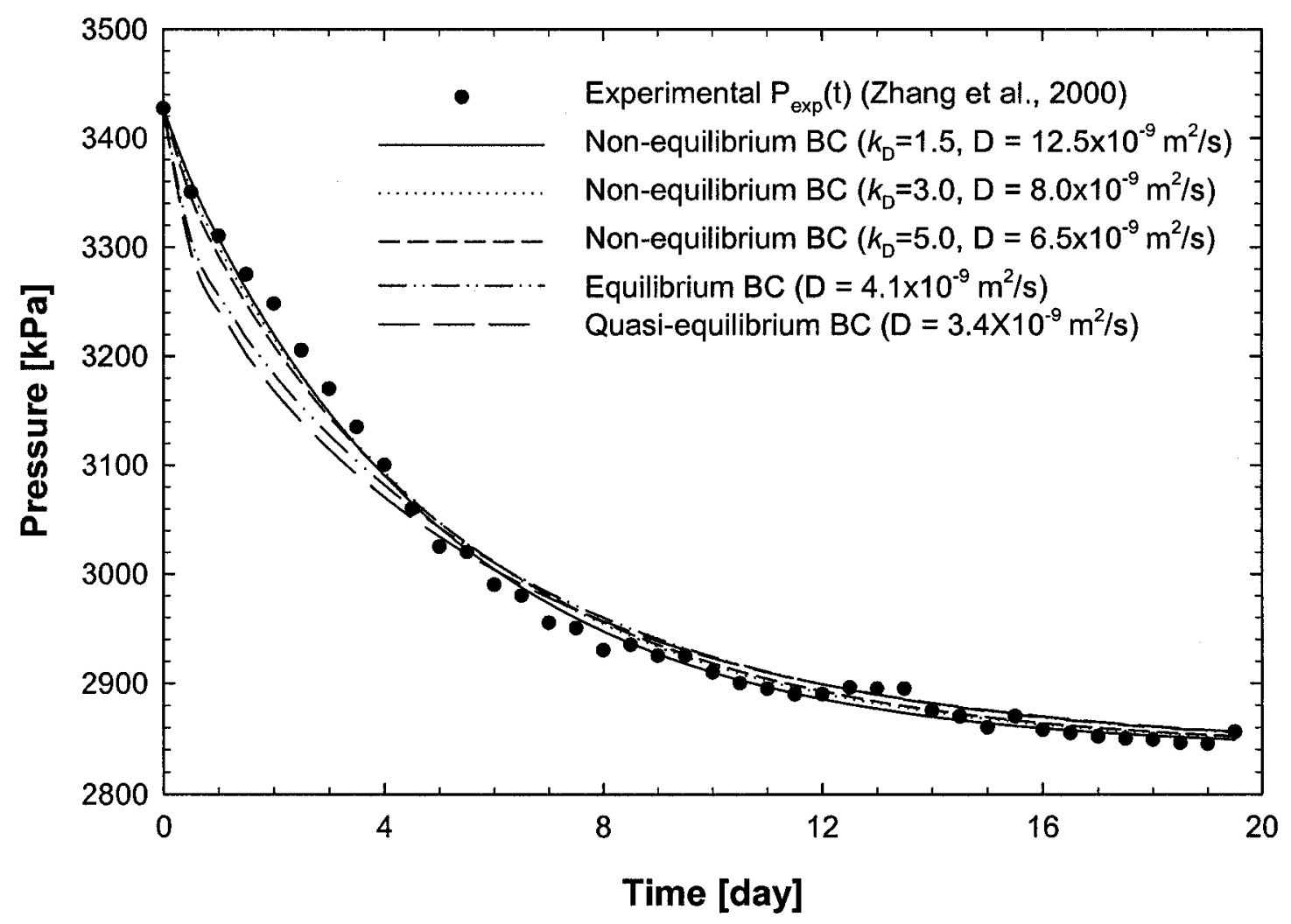

Figure 5.4 History matching of the calculated pressure vs. time curves for the three different BCs (Equilibrium, Quasi-equilibrium and Non-equilibrium) with the experimental data for $\mathrm{CO}_{2}$-heavy oil system (Zhang et al., 2000). 
measured pressures are larger at the initial stage than those at the final stage close to the equilibrium state. The reason for larger pressure differences at the beginning is that the solvent-heavy oil interface cannot be saturated instantaneously, which is assumed in the equilibrium and quasi-equilibrium $\mathrm{BCs}$. When the non-equilibrium $\mathrm{BC}$ is applied, the pressure differences are comparatively smaller during the entire diffusion process. Overall, the $P_{\text {car }} \sim t$ curves predicted by using the non-equilibrium $\mathrm{BC}$ better fit the experimental data than those calculated by using the other two BCs. This indicates that the non-equilibrium $\mathrm{BC}$ can be used to best describe the diffusion process for $\mathrm{CO}_{2}$-heavy oil system. However, it is also seen from Fig. 5.4 that, when the non-equilibrium $\mathrm{BC}$ is applied, the calculated $P_{\text {cal }} \sim t$ curves for several combinations of $D$ and $k_{\mathrm{D}}$ are close to each other. Thus with the non-equilibrium $\mathrm{BC}$, one cannot uniquely determine the optimal $D$ and $k_{\mathrm{D}}$.

Based on the minimum $\Delta P_{\text {ave }}$ values shown in Figs. 5.1 and 5.2, and the $P_{\text {cal }} \sim t$ curves shown in Fig. 5.4 for the three BCs, it becomes obvious that the non-equilibrium $\mathrm{BC}$ is more applicable for carbon dioxide-heavy oil system than the equilibrium and quasi-equilibrium BCs. This may be correct as the heavy oil may offer strong interfacial resistance to the non-hydrocarbon solvent. In practice, it takes a long time for $\mathrm{CO}_{2}$ to reach its complete saturation in the heavy oil phase. Furthermore, the experimental pressure decay for $\mathrm{CO}_{2}$-heavy oil system is about $17 \%(\sim 590 \mathrm{kPa})$ of the initial pressure (Zhang et al., 2000). Therefore, the solvent-heavy oil interface is not instantaneously saturated with the solvent under the so-called equilibrium pressure during the experiment. Accordingly, the equilibrium $\mathrm{BC}$ and quasi-equilibrium $\mathrm{BC}$ may not be suitable for $\mathrm{CO}_{2}$ heavy oil system. 


\subsubsection{Methane-heavy oil system}

The diffusivity data for methane-heavy oil system obtained in this study and published previously at the termination pressure of $3,420 \mathrm{kPa}$ and $T=21^{\circ} \mathrm{C}$ are compared in Table 5.3. Figure 5.5 shows the $\Delta P_{\text {ave }}$ vs. $D$ curves for $\mathrm{CH}_{4}$-heavy oil system under the three $\mathrm{BCs}$, in which four $k_{\mathrm{D}}$ values are chosen for the non-equilibrium $\mathrm{BC}$. The minimum $\Delta P_{\text {ave }}$ values for all the three $\mathrm{BCs}$ are found to be about $1.80 \mathrm{kPa}$. The corresponding diffusion coefficients determined from the history matching are equal to $16.1 \times 10^{-9}$, $16.0 \times 10^{-9}$ and $16.5 \times 10^{-9} \mathrm{~m}^{2} / \mathrm{s}\left(k_{\mathrm{D}}=100\right)$ for the equilibrium, quasi-equilibrium and nonequilibrium boundary conditions, respectively.

The above three diffusion coefficients obtained for the three BCs are very close to each other. More specifically, it is found that, in order to obtain similar minimum $\Delta P_{\text {ave }}$ value, the non-equilibrium boundary condition can be applied only if a sufficiently large mass-transfer Biot number $k_{\mathrm{D}}$ is chosen. Physically, the non-equilibrium $\mathrm{BC}$ with sufficiently large $k_{\mathrm{D}}$ values is close to the equilibrium $\mathrm{BC}\left(k_{\mathrm{D}}=\infty\right)$. The equilibrium $\mathrm{BC}$ means that $\mathrm{CH}_{4}$-heavy oil interface offers no resistance to the mass transfer and that the interface is saturated with methane at the equilibrium pressure instantaneously. Methaneheavy oil system has this peculiar behaviour probably because methane belongs to the same family of hydrocarbons. In most cases, methane actually coexists with heavy oil before it is recovered. Therefore, methane saturates the interface immediately after it is made in contact with heavy oil. It is also generally anticipated that the hydrocarbon solvents, such as methane, ethane, propane and butane, diffuse more readily across the solvent-heavy oil interface than non-hydrocarbon solvents, such as carbon dioxide, flue gas and nitrogen. Furthermore, it should also be noted that the experimental pressure 
Table 5.3 Comparison of the results obtained in this study with the previously published data for $\mathrm{CH}_{4}$-heavy oil system (Zhang et al., 2000) at $T=21^{\circ} \mathrm{C}$.

\begin{tabular}{|l|l|c|c|}
\hline \multicolumn{1}{|c|}{ Reference } & Boundary condition & $\begin{array}{c}\text { Diffusion coefficient } \\
D\left[10^{-9} \mathrm{~m}^{2} / \mathrm{s}\right]\end{array}$ & $\begin{array}{c}\text { Biot number } \\
k_{\mathrm{D}}\end{array}$ \\
\hline \multirow{3}{*}{ This study } & Equilibrium & 16.1 & - \\
\cline { 2 - 4 } & Quasi-equilibrium & 16.0 & - \\
\cline { 2 - 4 } & Non-equilibrium & 16.5 & - \\
\hline Zhang et al. (2000) & Equilibrium & 8.6 & 1.461 \\
\hline Civan and Rasmussen (2002) & Non-equilibrium & 0.22 & 67.89 \\
\hline Civan and Rasmussen (2003) & Non-equilibrium & 0.50 & \\
\hline
\end{tabular}




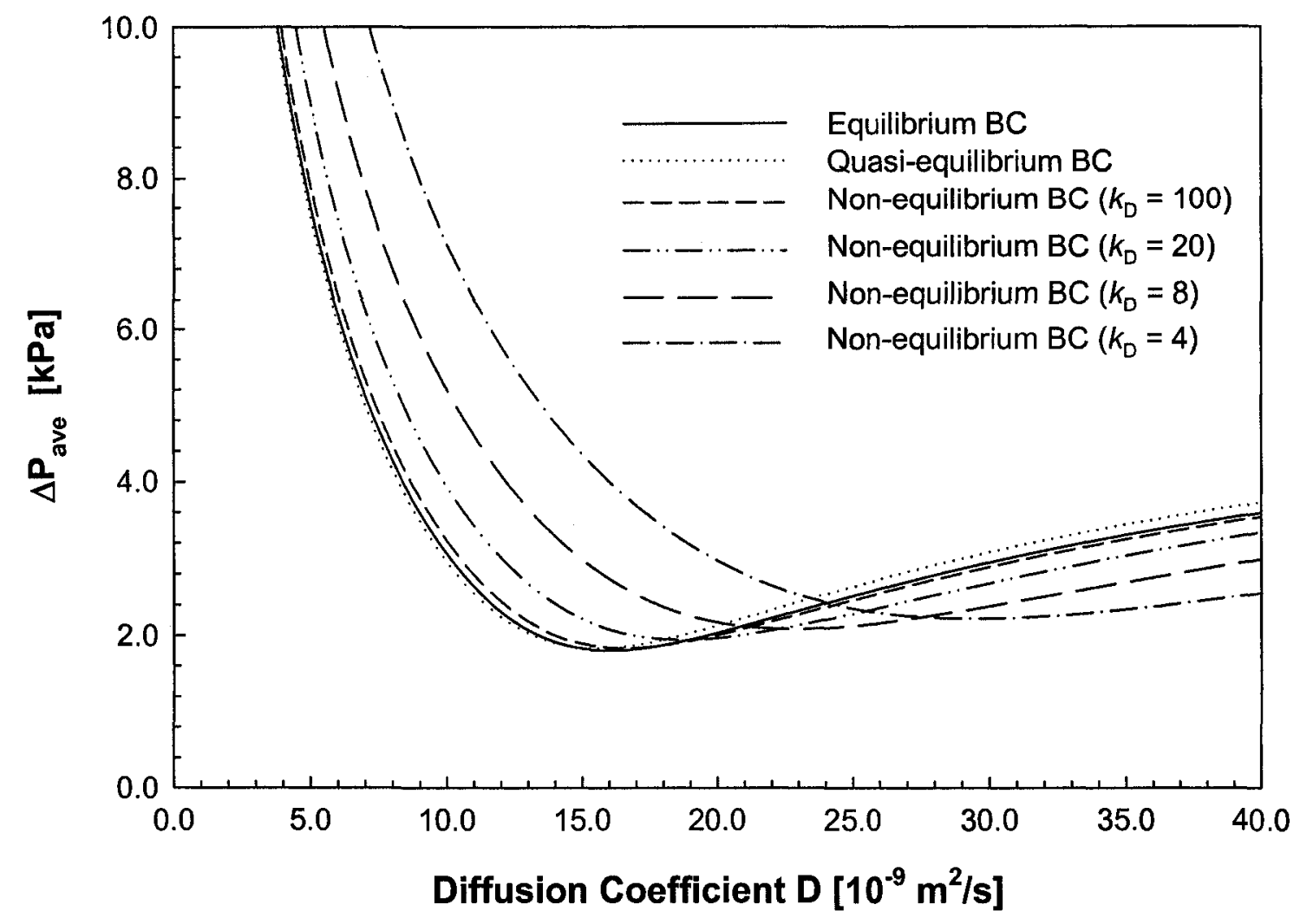

Figure 5.5 Variations of the average pressure difference $\Delta P_{\text {ave }}$ with the diffusion coefficient $D$ under the equilibrium, quasi-equilibrium and non-equilibrium boundary conditions for $\mathrm{CH}_{4}$-heavy oil system based on the pressure decay data published by Zhang et al. (2000). 
decay for $\mathrm{CH}_{4}$-heavy oil system is about $1.5 \%(\sim 50 \mathrm{kPa})$ of the initial pressure (Zhang et al., 2000). This small pressure decay can be considered as another reason why the equilibrium $\mathrm{BC}$ is most suitable for describing the mass transfer across $\mathrm{CH}_{4}$-heavy oil interface.

The detailed results of the history matching of the calculated pressures with the measured pressures for all the three BCs are shown in Fig. 5.6. This figure shows that, the calculated $P_{\text {cal }} \sim t$ curves for all the three BCs are very close to each other. Hence, any one of the three BCs can be applied at the methane-heavy oil interface throughout the diffusion process in order to determine the diffusion coefficient. Particularly, when the non-equilibrium $\mathrm{BC}$ is assumed at the interface, a sufficiently large value of mass-transfer Biot number should be chosen for this system. Moreover, it should be noted that the pressure decay within the initial 0.5 days is much larger in comparison with that at a later time. This implies that more methane diffuse into heavy oil at the beginning and that $\mathrm{CH}_{4}$-heavy oil interface offers no resistance to the diffusion of methane in heavy oil. Based on the above analyses, the equilibrium $\mathrm{BC}$ is suitable for methane-heavy oil system.

\subsection{Determination of Diffusion Coefficients Based on the Measured Pressure Decay}

\section{Data in this Study}

\subsubsection{Equilibrium pressures}

As a first step in the diffusivity determination, the equilibrium pressure, $P_{\text {eq }}$, of each experimental run is calculated from the measured solubility, $\chi(P, T)$, of the corresponding solvent-heavy oil system at $T=23.9^{\circ} \mathrm{C}$. The detailed solubility measurement results of all 


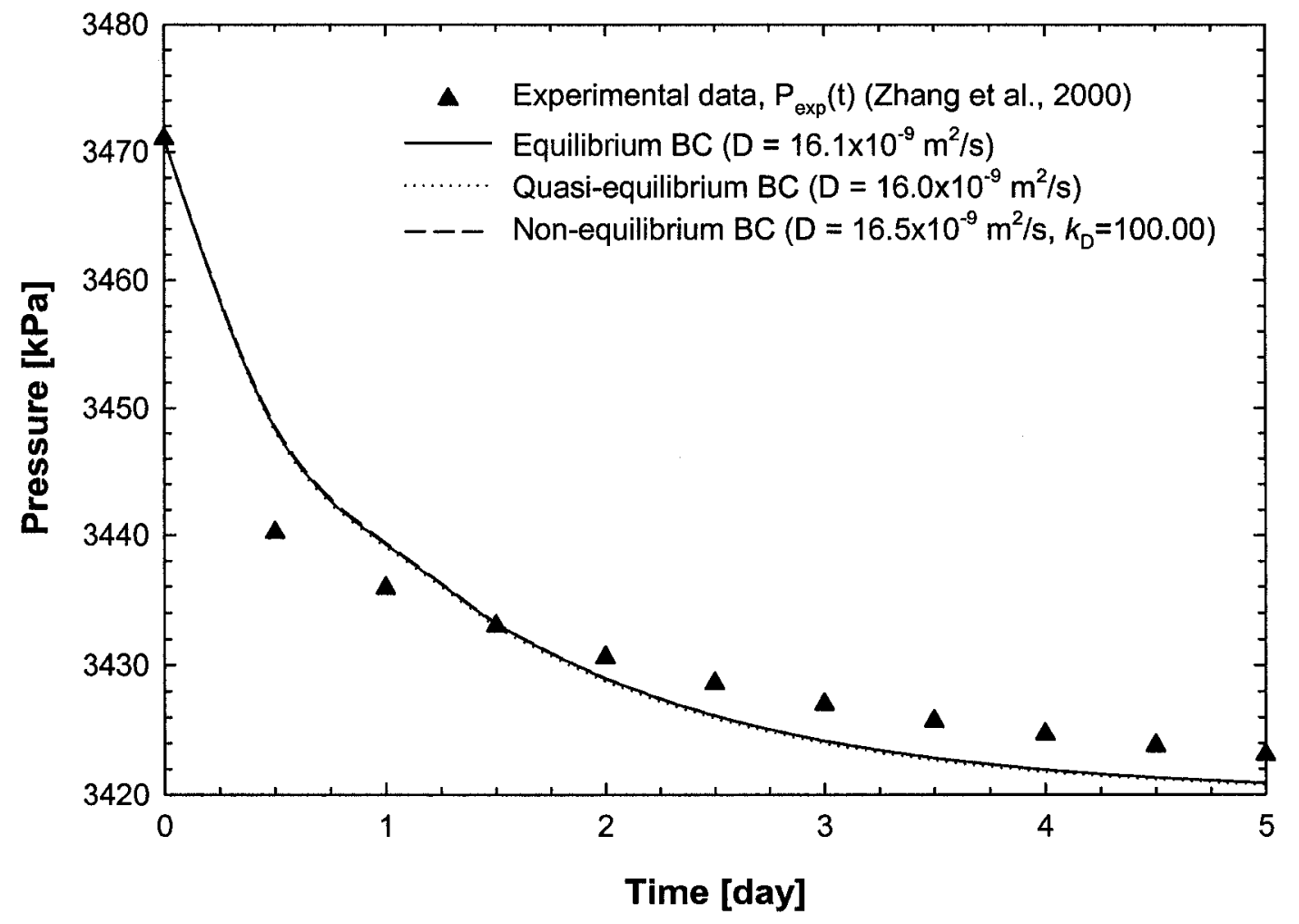

Figure 5.6 History matching of the calculated pressure vs. time curves for the three different BCs (Equilibrium, Quasi-equilibrium and Non-equilibrium) with the experimental data for $\mathrm{CH}_{4}$-heavy oil system (Zhang et al., 2000). 
the three solvent-heavy oil systems are given in Section 4.3.4. Furthermore, the complete description about the equilibrium pressure calculation from such measured solubility for each pressure decay test is presented in Section 3.3. From such determined equilibrium pressure, the number of moles of solvent remaining in the gas phase, $N_{\text {eq }}$, at the equilibrium pressure $P_{\text {eq }}$, is computed from Eq. [3.18b]. Then in terms of conservation of solvent mass in a closed system, the solvent saturation concentration in heavy oil is found to be $c_{\text {sat }}\left(P_{\text {eq }}\right)=\left(N_{\mathrm{i}}-N_{\text {eq }}\right) / V_{\text {oil }}$.

The measured solubility data and the results of the equilibrium pressure calculation for each pressure decay test are shown in Fig. 5.7 for carbon dioxide-heavy oil system, Fig. 5.8 for methane-heavy oil system, and Fig 5.9 for propane-heavy oil system. Here, it is worthwhile to point out that determining the equilibrium pressure by using this new strategy is a major improvement made for this pressure decay method in the determination of diffusion coefficient (Tharanivasan et al., 2004b; 2004c). Thus, there is no need to measure the pressure decay data for an unreasonably long time until the equilibrium pressure is reached. It should also be noted that, though in this study the pressure decay test for each solvent-heavy oil system lasts for a sufficiently long period, its termination pressure is still slightly higher than its corresponding equilibrium pressure (See Table 4.1 and Figs. 5.7-5.9).

\subsubsection{Carbon dioxide-heavy oil system}

The measured pressure decay data for both Run-1 and Run-2 of carbon dioxideheavy oil system are plotted in Figs. 5.10a and 5.10b, respectively. These plots include the best-fit curves of the measured data at different diffusion times, i.e., $t=5,10,20$ and 37.3 days for Run- 1 and $t=5$ and 10.6 days for Run-2. The diffusion time represents the 


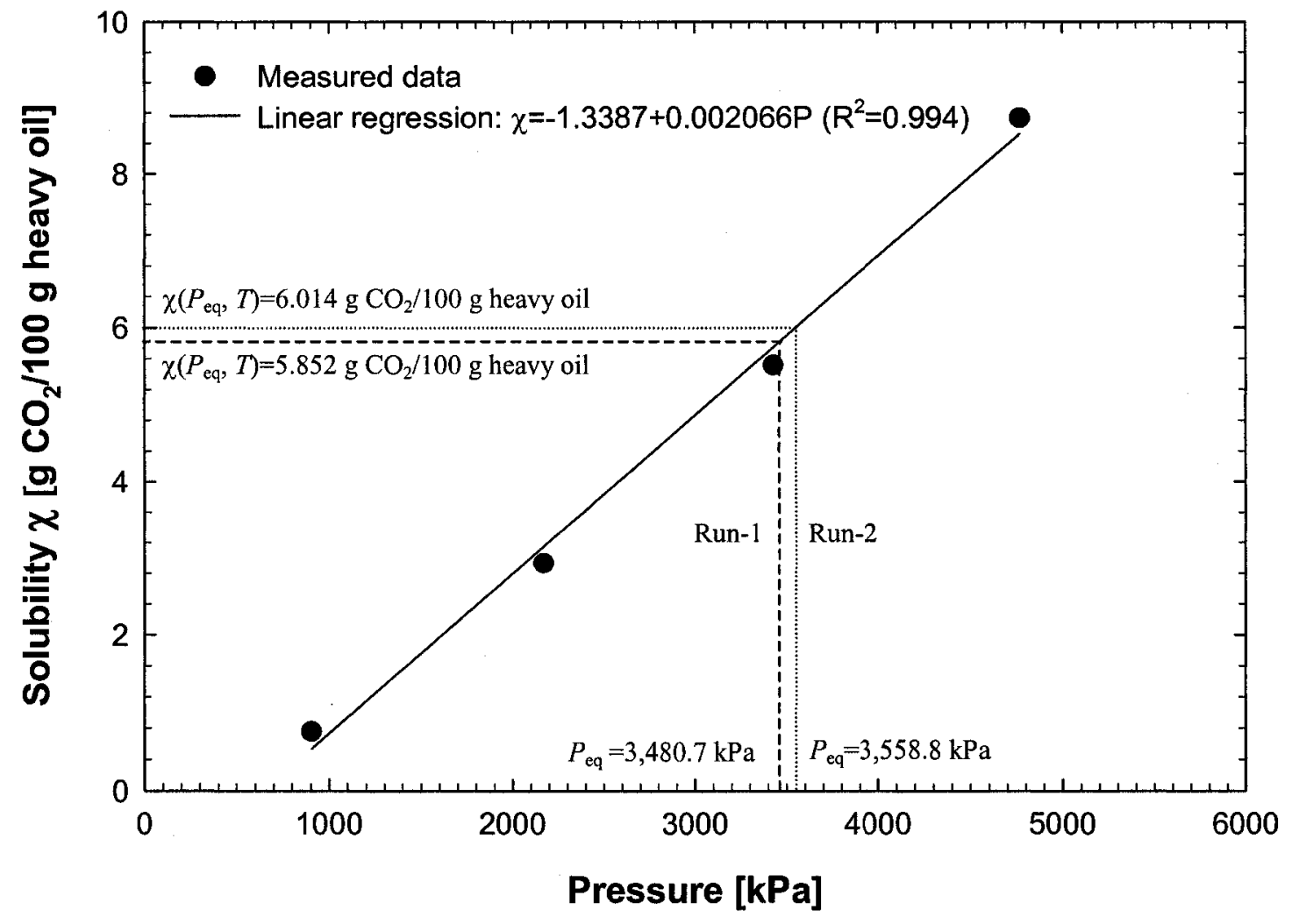

Figure 5.7 The equilibrium pressures determined from the measured solubility data for $\mathrm{CO}_{2}$-heavy oil system at $T=23.9^{\circ} \mathrm{C}$. 


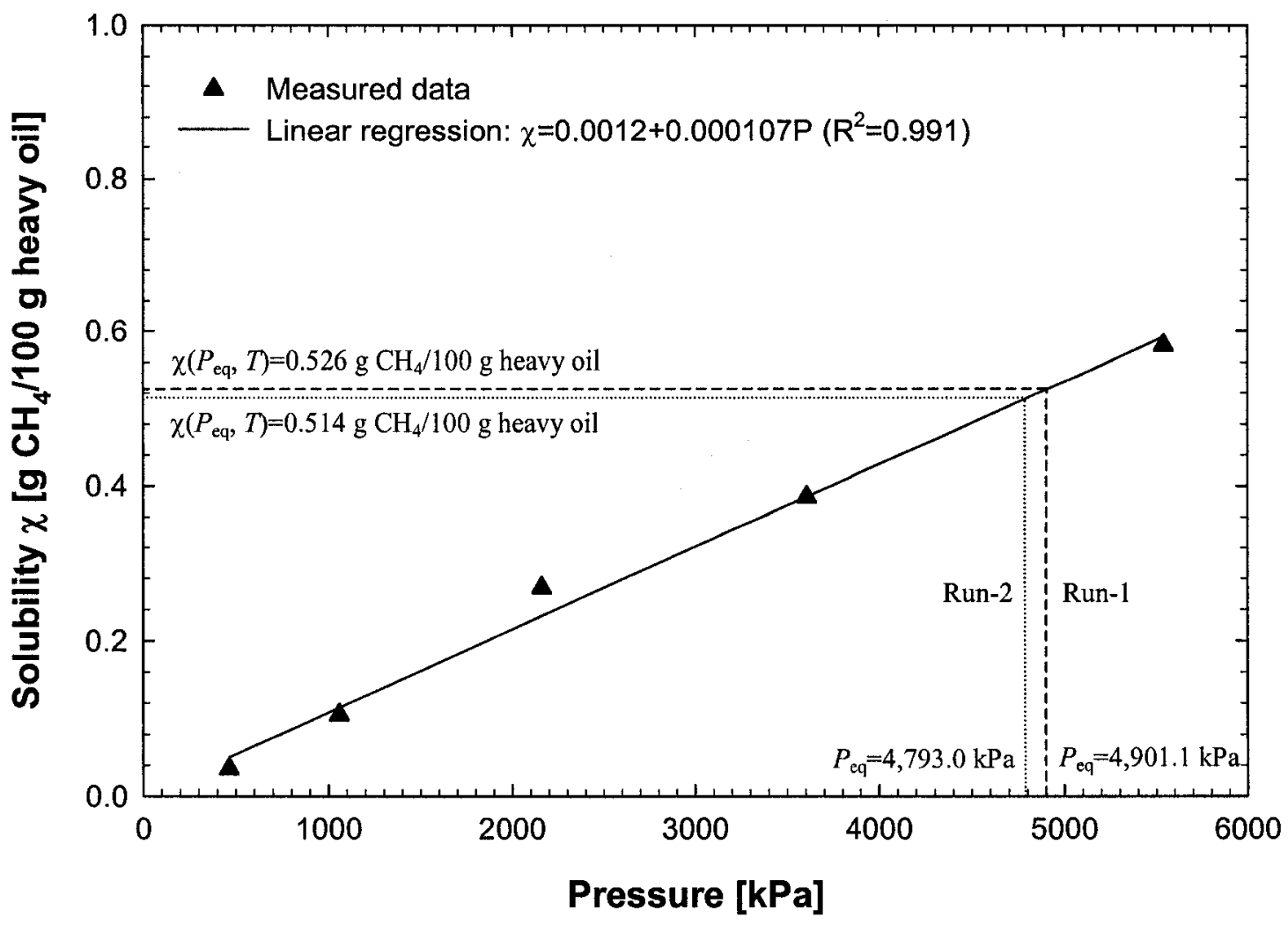

Figure 5.8 The equilibrium pressures determined from the measured solubility data for $\mathrm{CH}_{4}$-heavy oil system at $T=23.9^{\circ} \mathrm{C}$. 


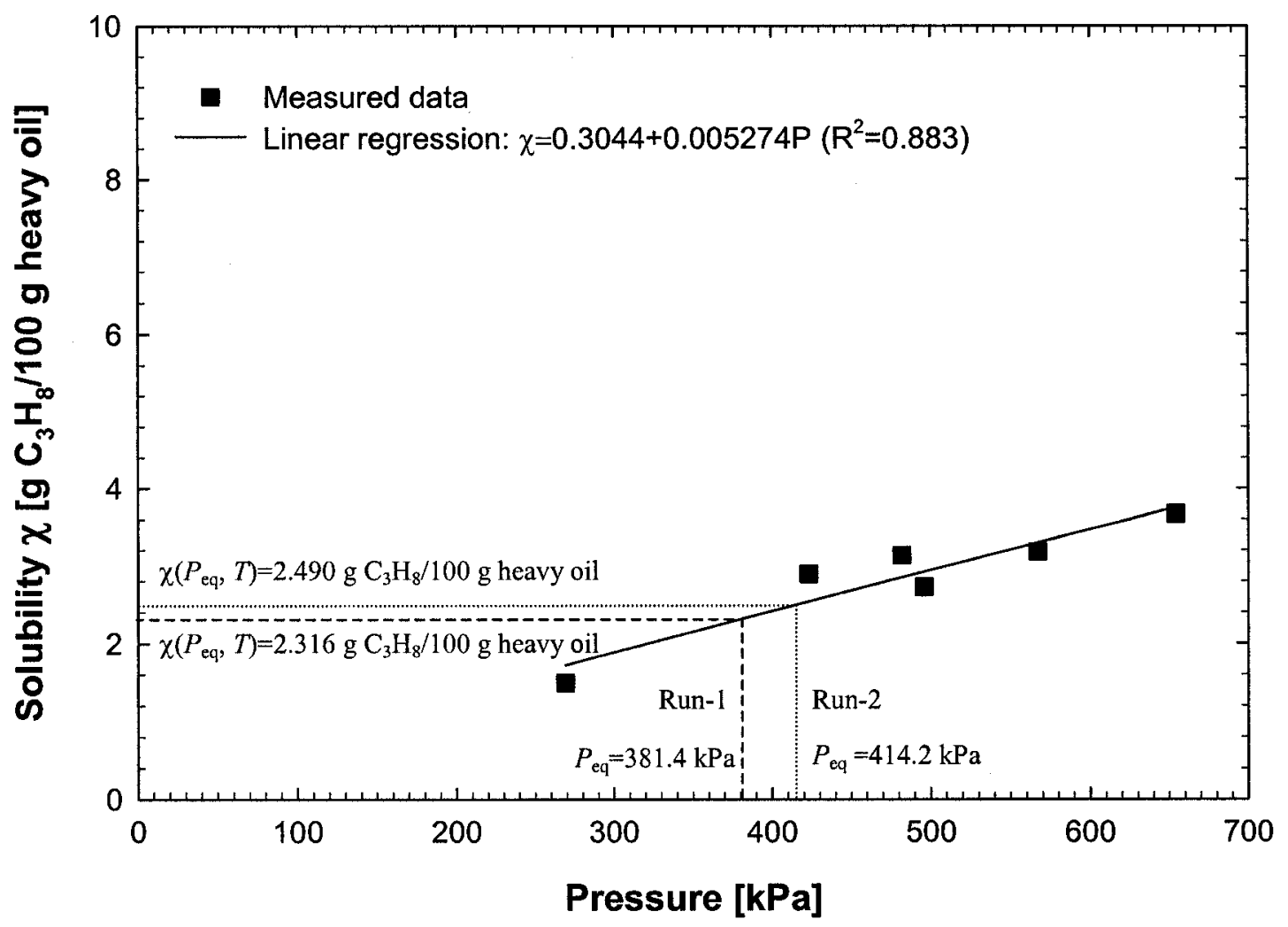

Figure 5.9 The equilibrium pressures determined from the measured solubility data for $\mathrm{C}_{3} \mathrm{H}_{8}$-heavy oil system at $T=23.9^{\circ} \mathrm{C}$. 


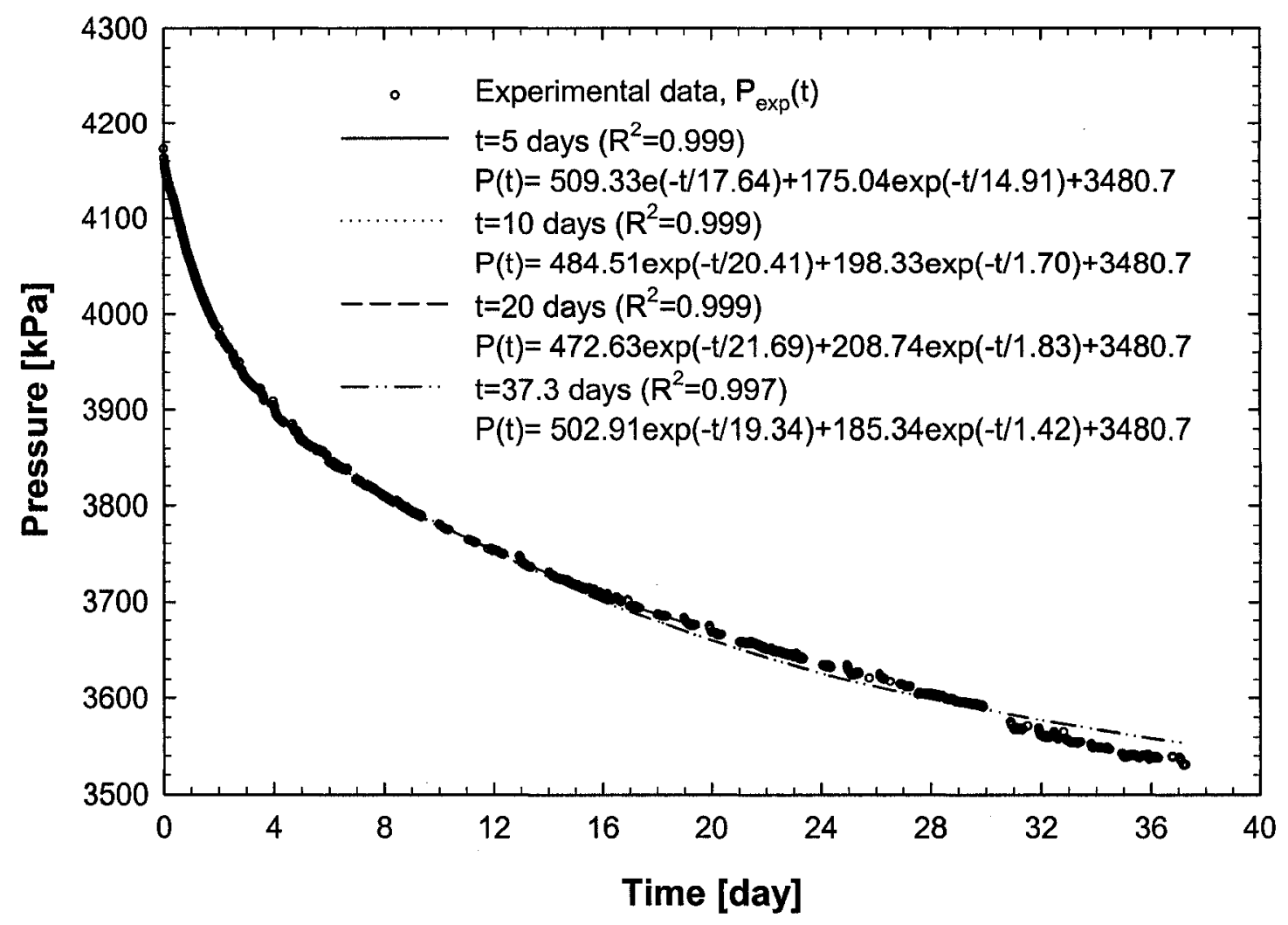

Figure 5.10a Measured pressure decay data and the best-fit curves at different diffusion times for $\mathrm{CO}_{2}$-heavy oil system (Run-1) at $T=23.9^{\circ} \mathrm{C}$. 


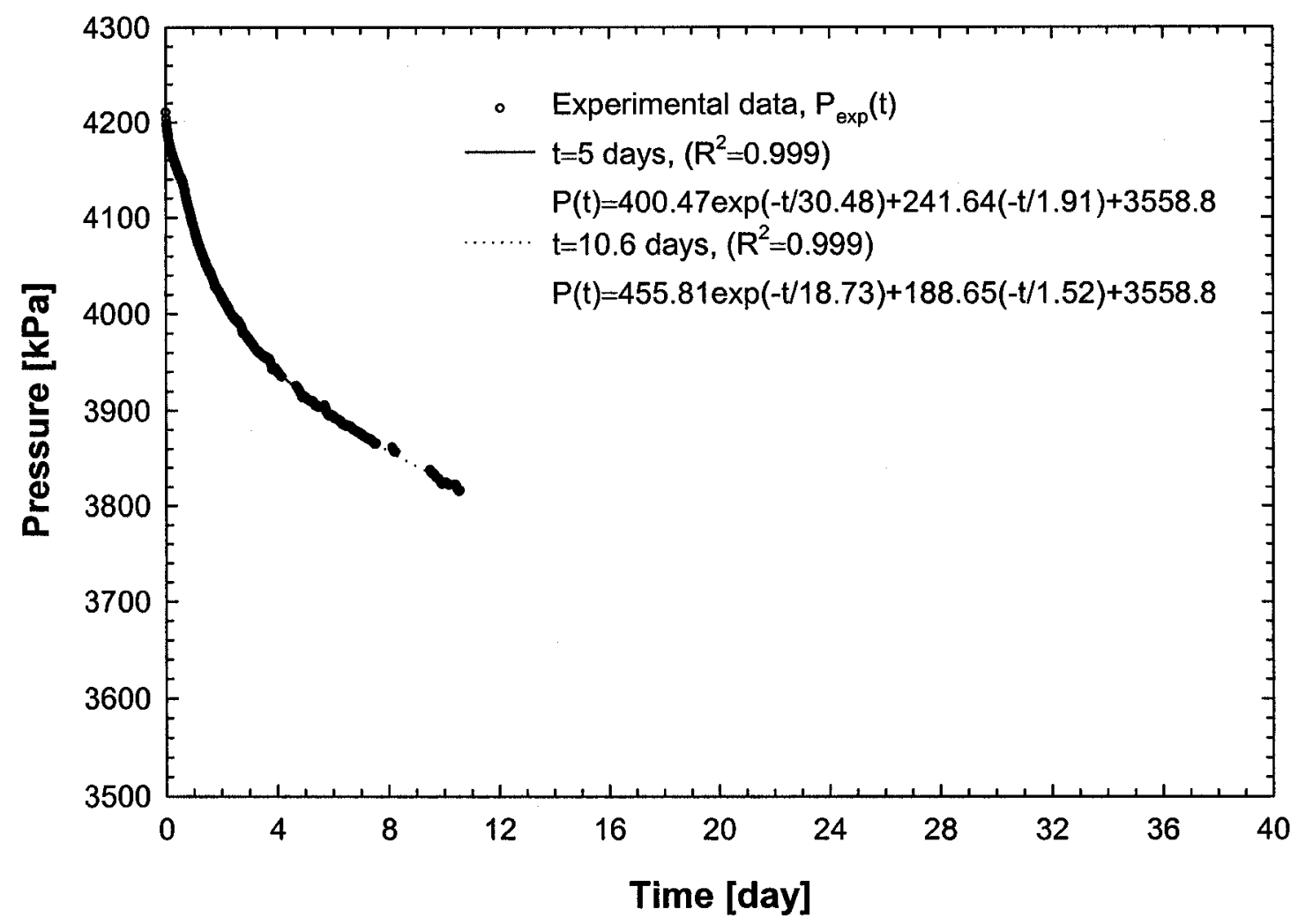

Figure 5.10b Measured pressure decay data and the best-fit curves at different diffusion times for $\mathrm{CO}_{2}$-heavy oil system (Run-2) at $T=23.9^{\circ} \mathrm{C}$. 
duration in which the measured data are used to find the best-fit curve. Here, Equation [3.12] along with the known equilibrium pressure, $P_{\text {eq }}$, from the previous section, is used to do the best-curve fitting (Wittink, 1988). The non-linear regression yields excellent correlation coefficients $\left(R^{2} \geq 0.997\right)$ for these two experimental runs of $\mathrm{CO}_{2}$-heavy oil system. This indicates that the assumed expression for pressure as a function of time in Eq. [3.12] is an excellent approximation for the present system. From this non-linear regression of the measured pressure decay data for each diffusion time, the corresponding parameters, $m_{1}, m_{2}, k_{1}$ and $k_{2}$ defined in Eq. [3.12], are found. The values of these parameters for different diffusion times are given in the regressed equations, which are also included in Figs. 5.10a and 5.10b.

The diffusion coefficients of carbon dioxide in heavy oil at different diffusion times for each of the three different $\mathrm{BCs}$ are given in Table 5.4a for Run-1 and Table 5.4b for Run-2, respectively. It is noted from these two tables that, in general, the diffusivity slightly decreases with increase in the diffusion time for each $\mathrm{BC}$. In addition, when the non-equilibrium $\mathrm{BC}$ is applied at the interface, the value of mass-transfer Biot number, $k_{\mathrm{D}}$, increases with the diffusion time. This implies that the resistance to mass transfer at the interface decreases as the diffusion proceeds. The variations of the diffusivity and the mass-transfer Biot number with the diffusion time can also be attributed to the changes of the heavy oil properties due to the gradual dissolution of solvent (Upreti and Mehrotra, 2002).

If the minimum objective functions $\Delta P_{\text {ave }}$ given in Table $5.4 \mathrm{a}$ and Table $5.4 \mathrm{~b}$ for three different BCs at the diffusion time of $t=5$ days are compared, the non-equilibrium $\mathrm{BC}$ gives the best history matching (the smallest $\Delta P_{\text {ave }}$ ) of the calculated pressure decay 
Table 5.4a. Comparison of diffusion coefficients at different diffusion times for $\mathrm{CO}_{2^{-}}$ heavy oil system (Run-1) at $T=23.9^{\circ} \mathrm{C}$.

\begin{tabular}{|c|c|c|c|c|}
\hline $\begin{array}{c}\text { Diffusion time } \\
\text { [day }]\end{array}$ & Boundary condition & $\begin{array}{c}\text { Diffusivity } \\
D\left[10^{-9} \mathrm{~m}^{2} / \mathrm{s}\right]\end{array}$ & $\begin{array}{c}\text { Biot number } \\
k_{\mathrm{D}}\end{array}$ & $\begin{array}{c}\Delta P_{\text {ave }} \\
{[\mathrm{kPa}]}\end{array}$ \\
\hline \multirow{4}{*}{5} & Equilibrium & 0.72 & - & 11.0 \\
\cline { 2 - 5 } & Quasi-equilibrium & 0.52 & - & 11.8 \\
\cline { 2 - 5 } & Non-equilibrium & 0.94 & 19.20 & 6.5 \\
\hline \multirow{4}{*}{10} & Equilibrium & 0.67 & - & 11.7 \\
\cline { 2 - 5 } & Quasi-equilibrium & 0.53 & - & 10.7 \\
\cline { 2 - 5 } & Non-equilibrium & 0.70 & $>80$ & $<11.9$ \\
\hline \multirow{3}{*}{20} & Equilibrium & 0.59 & - & 20.0 \\
\cline { 2 - 5 } & Quasi-equilibrium & 0.49 & - & 17.5 \\
\cline { 2 - 5 } & Non-equilibrium & 0.62 & $>100$ & $<21.0$ \\
\hline \multirow{5}{*}{37.3} & Equilibrium & 0.56 & - & 19.9 \\
\cline { 2 - 5 } & Quasi-equilibrium & 0.46 & - & 16.6 \\
\cline { 2 - 5 } & Non-equilibrium & 0.57 & $>100$ & $<20.9$ \\
\hline
\end{tabular}


Table 5.4b. Comparison of diffusion coefficients at different diffusion times for $\mathrm{CO}_{2}$ heavy oil system (Run-2) at $T=23.9^{\circ} \mathrm{C}$.

\begin{tabular}{|c|c|c|c|c|}
\hline $\begin{array}{c}\text { Diffusion time } \\
{[\text { day }]}\end{array}$ & Boundary condition & $\begin{array}{c}\text { Diffusivity, } \\
D\left[10^{-9} \mathrm{~m}^{2} / \mathrm{s}\right]\end{array}$ & $\begin{array}{c}\text { Biot number } \\
k_{\mathrm{D}}\end{array}$ & $\begin{array}{c}\Delta P_{\text {ave }} \\
{[\mathrm{kPa}]}\end{array}$ \\
\hline \multirow{3}{*}{5} & Equilibrium & 0.72 & - & 11.0 \\
\cline { 2 - 5 } & Quasi-equilibrium & 0.56 & - & 12.0 \\
\cline { 2 - 5 } & Non-equilibrium & 0.92 & 19.90 & 7.4 \\
\hline \multirow{3}{*}{10.6} & Equilibrium & 0.65 & - & 13.3 \\
\cline { 2 - 5 } & Quasi-equilibrium & 0.53 & - & 11.9 \\
\cline { 2 - 5 } & Non-equilibrium & 0.66 & $>375$ & $<13.4$ \\
\hline
\end{tabular}


with the measured pressure decay $\left(\Delta P_{\text {ave }}=6.5 \mathrm{kPa}\right.$ for Run-1 and $\Delta P_{\mathrm{ave}}=7.4 \mathrm{kPa}$ for Run-2). Also for this $\mathrm{BC}$, it is found that the mass-transfer Biot number $k_{\mathrm{D}}$ increases with the diffusion time. This indicates that the resistance to mass transfer at the solvent-heavy oil interface, $1 / k$, is dominant only at early diffusion stages. At large diffusion times, i.e., $t=10,20$ and 37.3 days for Run- 1 and $t=10.6$ days for Run-2, in terms of the minimum $\Delta P_{\text {ave, }}$ nevertheless, the quasi-equilibrium $\mathrm{BC}$ gives the best history matching of the calculated pressures with the measured pressures in comparison with the other two BCs. This means that the solvent concentration at the interface varies with the existing pressure in the solvent phase at large times and follows the quasi-equilibrium BC. In summary, the non-equilibrium $\mathrm{BC}$ at $\mathrm{CO}_{2}$-heavy oil interface is better at the beginning and the quasiequilibrium $\mathrm{BC}$ is more suitable for carbon dioxide-heavy oil system at large times. This is expected because carbon dioxide may not saturate its interface with heavy oil instantaneously as it is a typical non-hydrocarbon solvent. Thus, the non-equilibrium $\mathrm{BC}$ should give the best result in the determination of diffusion coefficient of carbon dioxide in heavy oil at small times, while the quasi-equilibrium $\mathrm{BC}$ is more applicable for the solvent-heavy oil system at large times (Tharanivasan et al., 2004b).

At the diffusion time of $t=5$ days, the detailed comparisons of the calculated pressures under the three different BCs are compared with the measured pressures and shown in Fig. 5.11a for Run-1 and in Fig. 5.11b for Run-2. These figures clearly show that the differences between the calculated and measured pressures are larger at the beginning for both the equilibrium and quasi-equilibrium $\mathrm{BCs}$. This is because $\mathrm{CO}_{2}$ heavy oil interface cannot be saturated instantaneously, in contrast to the assumptions made in these two $\mathrm{BCs}$. When the non-equilibrium $\mathrm{BC}$ is applied, however, the pressure 


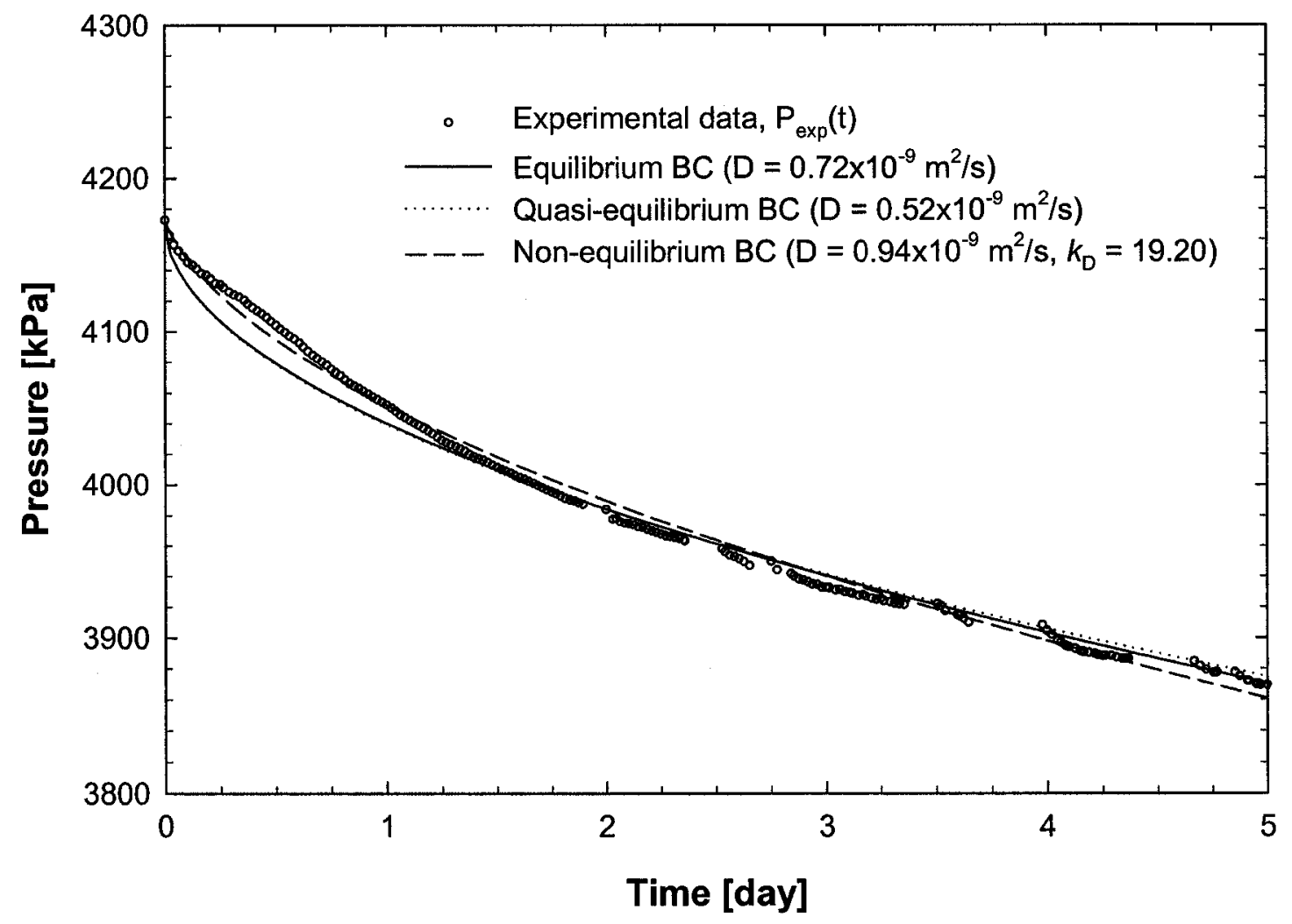

Figure 5.11a. History matching of the calculated pressures under three different BCs with the experimental data $\left(t=5\right.$ days) for $\mathrm{CO}_{2}$-heavy oil system (Run-1) at $T=23.9^{\circ} \mathrm{C}$. 


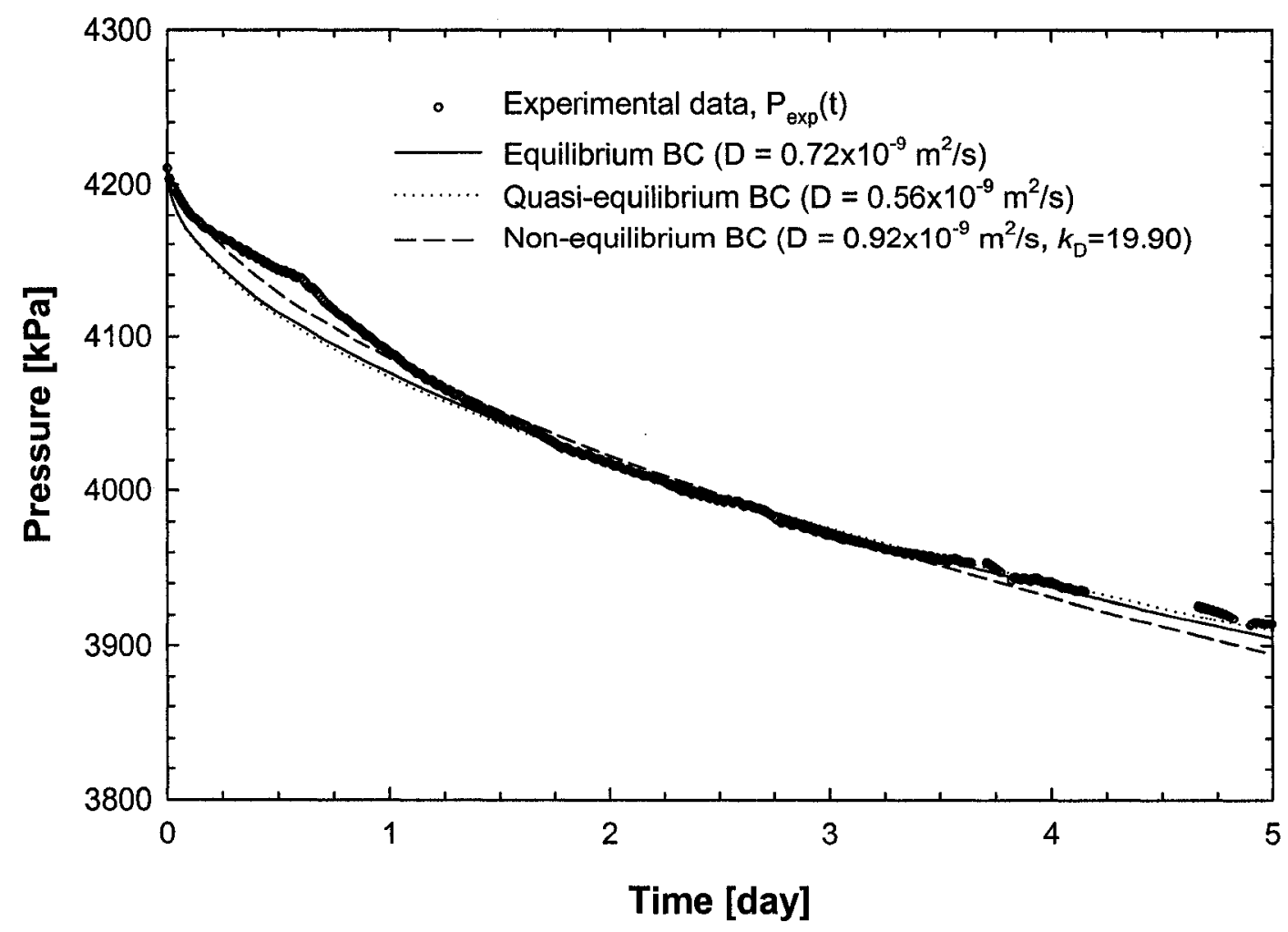

Figure 5.11b. History matching of the calculated pressures under three different BCs with the experimental data ( $t=5$ days) for $\mathrm{CO}_{2}$-heavy oil system (Run-2) at $T=23.9^{\circ} \mathrm{C}$. 
differences are much smaller. It should be noted that the non-equilibrium BC considers the interfacial resistance to the mass transfer. In this case, the diffusivity and masstransfer Biot number are found to be $0.94 \times 10^{-9} \mathrm{~m}^{2} / \mathrm{s}$ and 19.20 for Run- 1 , and $0.92 \times 10^{-9}$ $\mathrm{m}^{2} / \mathrm{s}$ and 19.90 for Run-2, respectively.

The dimensionless solvent concentration profiles in heavy oil at various times, $t=$ $0.5,1.0,2.0,5.0,10.0,15.0,20.0,30.0,37.3$ and 50.0 days, under the non-equilibrium $\mathrm{BC}$ determined from Run-1 are plotted in Fig. 5.12a for $\mathrm{CO}_{2}$-heavy oil system. Similarly, the dimensionless concentration profiles for Run- 2 at various diffusion times, $t=0.5,1.0$, $2.0,5.0,10.6,15.0,20.0,30.0$ and 50.0 days, under the non-equilibrium $\mathrm{BC}$ are plotted in Fig. $5.12 \mathrm{~b}$. In these figures, $X=0$ represents the bottom of the diffusion cell, whereas $X=1$ denotes the solvent-heavy oil interface. Comparison of different concentration profiles at different times shows that the solvent reaches the bottom of the diffusion cell within 1-2 days for both the experimental runs. On the other hand, it is also noted from the solvent concentration profile at $t=50.0$ days that the dimensionless solvent concentration in heavy oil at the bottom of the diffusion cell reaches about $99.0 \%$ in Fig. $5.12 \mathrm{a}$, and $99.5 \%$ in Fig 5.12b, respectively. This indicates that the complete saturation of heavy oil with $\mathrm{CO}_{2}$ and the corresponding equilibrium pressure can be achieved in an extremely long time. Alternatively, in this study, the equilibrium pressure is found from the measured solubility data at the same temperature for each solvent-heavy oil system. This new strategy not only provides a reliable way to accurately determine the equilibrium pressure, but also makes it possible to determine the solvent diffusivity in heavy oil by using the pressure decay data measured in a short period. 


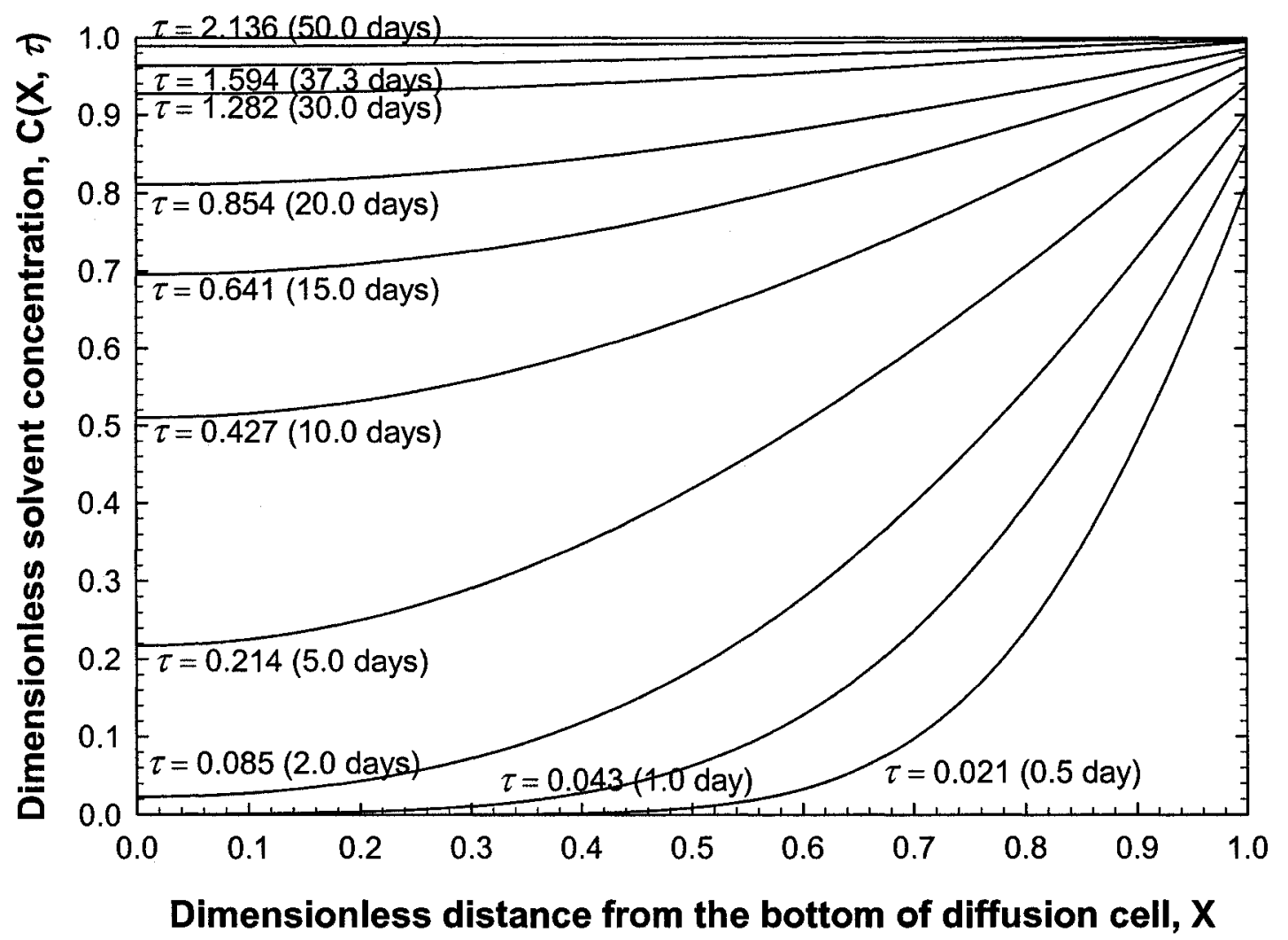

Figure 5.12a. Dimensionless solvent concentration profiles for $\mathrm{CO}_{2}$-heavy oil system (Run-1) at different times under the non-equilibrium boundary condition $\left(D=0.94 \times 10^{-9}\right.$ $\mathrm{m}^{2} / \mathrm{s}$ and $\left.k_{\mathrm{D}}=19.20\right)$. 


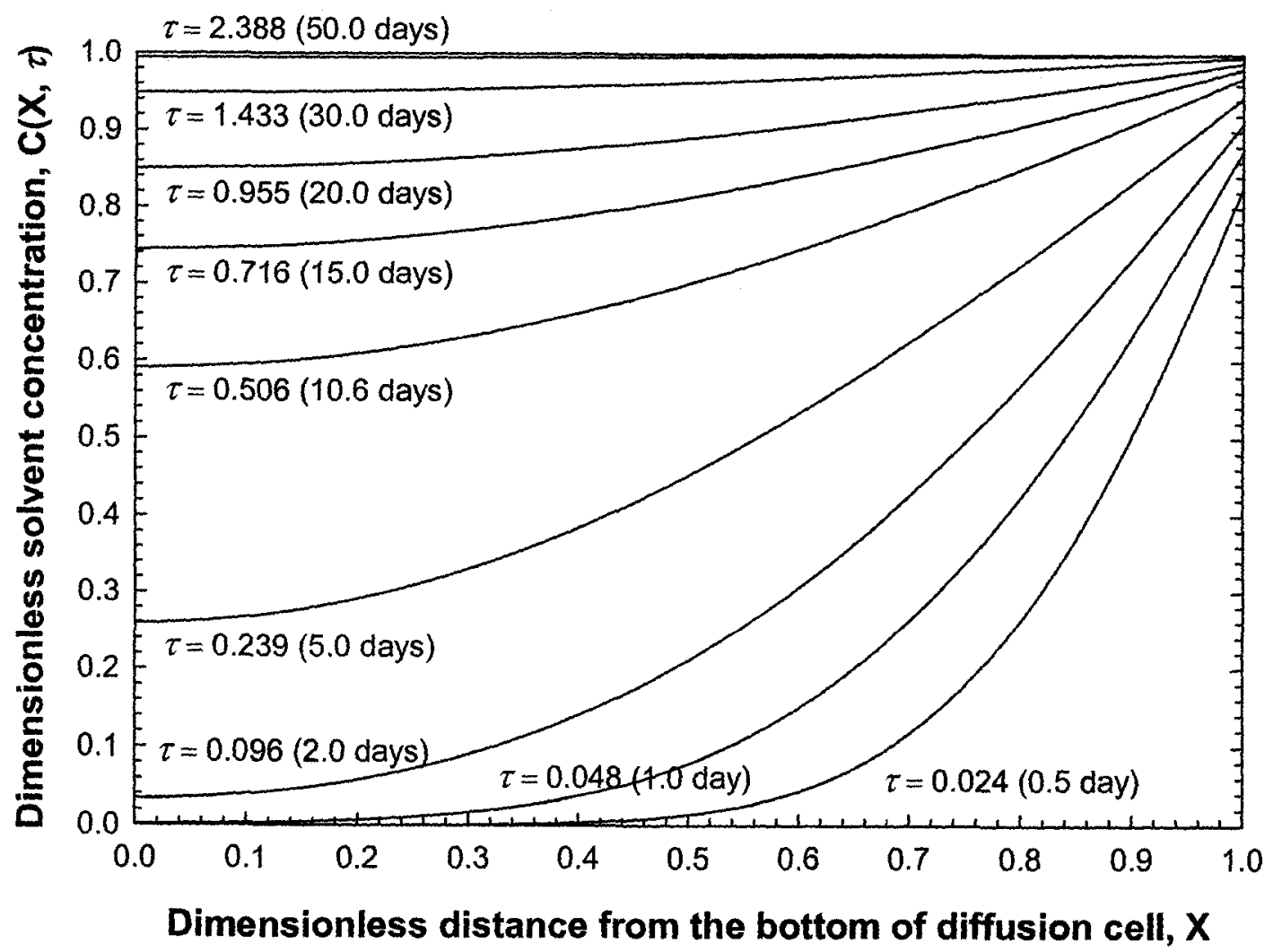

Figure 5.12b. Dimensionless solvent concentration profiles for $\mathrm{CO}_{2}$-heavy oil system (Run-2) at different times under the non-equilibrium boundary condition $\left(D=0.92 \times 10^{-9}\right.$ $\mathrm{m}^{2} / \mathrm{s}$ and $\left.k_{\mathrm{D}}=19.90\right)$. 


\subsubsection{Methane-heavy oil system}

The measured pressure decay data for both Run-1 and Run-2 of $\mathrm{CH}_{4}$-heavy oil system are plotted in Figs. 5.13a and 5.13b, respectively. These plots include the best-fit curves of the measured data at different diffusion times, i.e., $t=5,10,20$ and 23.8 days for Run-1 and $t=5,10,19.3$ and 26.3 days for Run-2. As discussed earlier, Equation [3.12] along with the known equilibrium pressure, $P_{\text {eq }}$, determined in the previous section 5.2.1 is used to find the best-fit curve for each diffusion time. The non-linear regression yields excellent correlation coefficients, $R^{2} \geq 0.997$ over longer diffusion times $(t \geq 10$ days), and $R^{2}=0.995$ for Run-1 and $R^{2}=0.993$ for Run-2 over a short diffusion time ( $t=$ 5 days). This indicates that the assumed expression for pressure as a function of time in Eq. [3.12] is an excellent approximation for $\mathrm{CH}_{4}$-heavy oil system. The values of the parameters, $m_{1}, m_{2}, k_{1}$ and $k_{2}$, for each diffusion time are given in the regressed equation, which is also included in Fig. 5.13a for Run-1 and in Fig. 5.13b for Run-2.

The diffusion coefficients of methane in heavy oil at four different diffusion times for the respective three different BCs are given in Table 5.5a for Run-1 and in Table 5.5b for Run-2. These two tables show that the determined diffusion coefficients are close to each other, independent of the boundary condition imposed at the methane-heavy oil interface. More specifically, the diffusivity data determined by using the equilibrium and quasi-equilibrium $\mathrm{BCs}$ are closer to each other because the total pressure decay due to the molecular diffusion for $\mathrm{CH}_{4}$-heavy oil system is very small. For example, in Run-1 experiment, the total pressure decay for methane-heavy oil system is about $2.3 \%$ of the initial pressure or $113.3 \mathrm{kPa}$, in comparison with the total pressure decay of $15.4 \%$ of the initial pressure or $642.5 \mathrm{kPa}$ for carbon dioxide-heavy oil system. Hence, there is no 


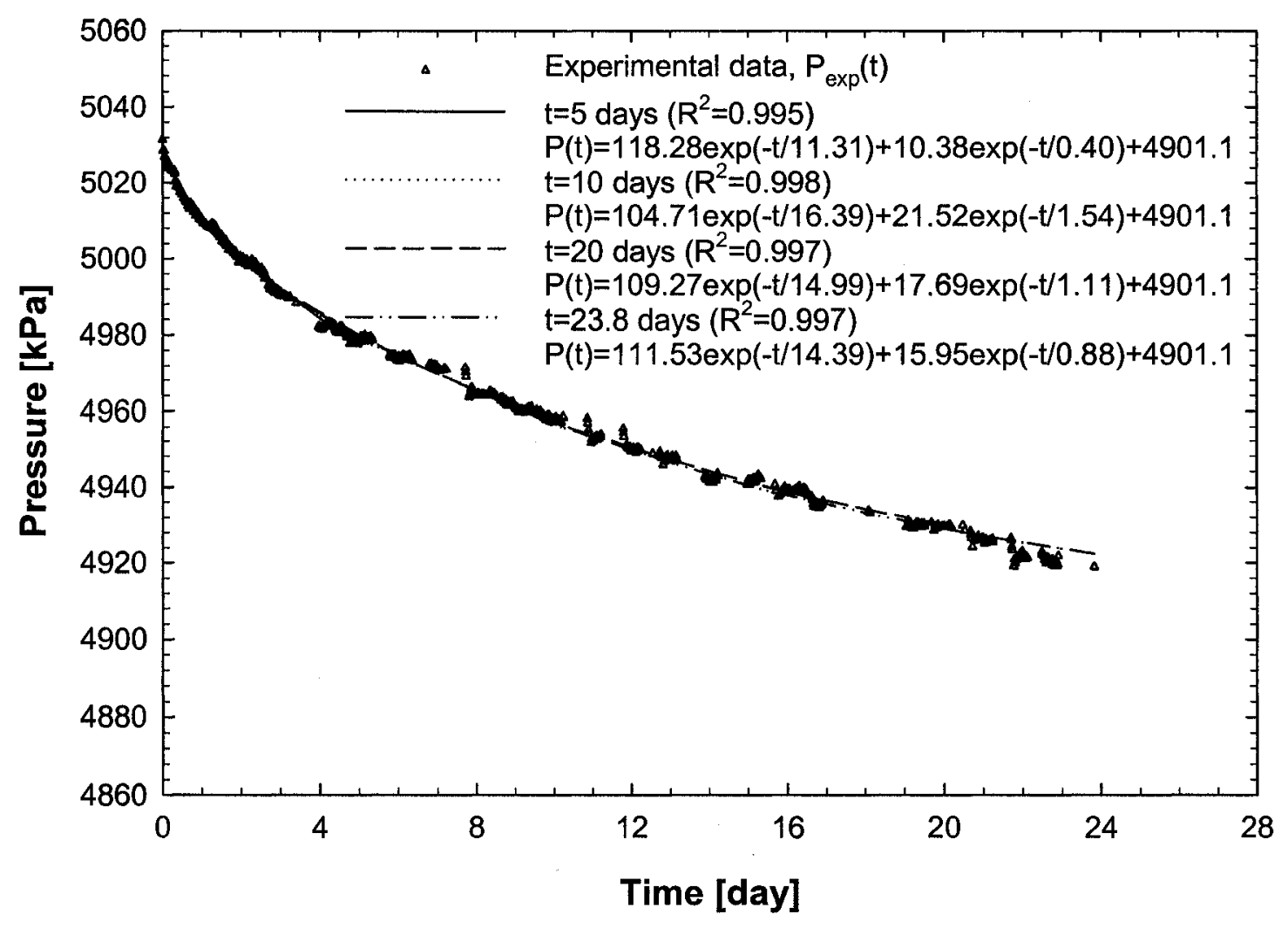

Figure 5.13a. Measured pressure decay data and the best-fit curves at different diffusion times for $\mathrm{CH}_{4}$-heavy oil system (Run-1) at $T=23.9^{\circ} \mathrm{C}$. 


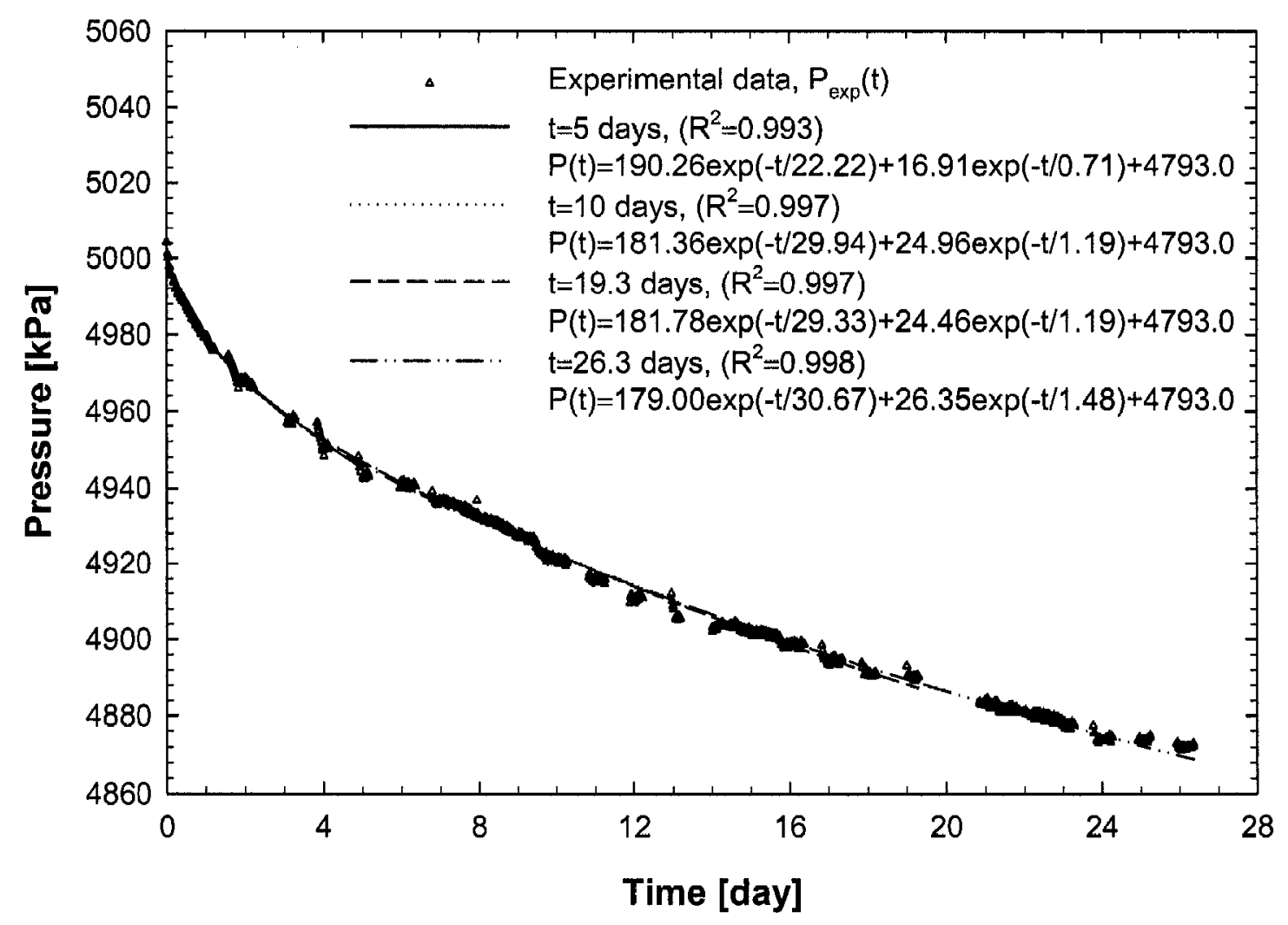

Figure 5.13b. Measured pressure decay data and the best-fit curves at different diffusion times for $\mathrm{CH}_{4}$-heavy oil system (Run-2) at $T=23.9^{\circ} \mathrm{C}$. 
Table 5.5a. Comparison of diffusion coefficients at different diffusion times for $\mathrm{CH}_{4}{ }^{-}$ heavy oil system (Run-1) at $T=23.9^{\circ} \mathrm{C}$.

\begin{tabular}{|c|c|c|c|c|}
\hline $\begin{array}{l}\text { Diffusion time } \\
\text { [day] }\end{array}$ & Boundary condition & $\begin{array}{c}\text { Diffusivity } \\
D\left[10^{-9} \mathrm{~m}^{2} / \mathrm{s}\right]\end{array}$ & $\begin{array}{l}\text { Biot number } \\
k_{\mathrm{D}}\end{array}$ & $\begin{array}{l}\Delta P_{\text {ave }} \\
{[\mathrm{kPa}]}\end{array}$ \\
\hline \multirow{3}{*}{5} & Equilibrium & 0.19 & - & 1.9 \\
\hline & Quasi-equilibrium & 0.19 & - & 2.0 \\
\hline & Non-equilibrium & 0.26 & 19.62 & 1.0 \\
\hline \multirow{3}{*}{10} & Equilibrium & 0.19 & - & 1.6 \\
\hline & Quasi-equilibrium & 0.19 & - & 1.6 \\
\hline & Non-equilibrium & 0.23 & 29 & 1.3 \\
\hline \multirow{3}{*}{20} & Equilibrium & 0.20 & - & 1.7 \\
\hline & Quasi-equilibrium & 0.19 & - & 1.8 \\
\hline & Non-equilibrium & 0.21 & 90 & 1.5 \\
\hline \multirow{3}{*}{23.8} & Equilibrium & 0.21 & - & 2.3 \\
\hline & Quasi-equilibrium & 0.20 & - & 2.3 \\
\hline & Non-equilibrium & 0.21 & 150 & 2.1 \\
\hline
\end{tabular}


Table 5.5b. Comparison of diffusion coefficients at different diffusion times for $\mathrm{CH}_{4}$ heavy oil system (Run-2) at $T=23.9^{\circ} \mathrm{C}$.

\begin{tabular}{|c|c|c|c|c|}
\hline $\begin{array}{l}\text { Diffusion time } \\
\text { [day] }\end{array}$ & Boundary condition & $\begin{array}{l}\text { Diffusivity, } \\
D\left[10^{-9} \mathrm{~m}^{2} / \mathrm{s}\right]\end{array}$ & $\begin{array}{c}\text { Biot number } \\
k_{\mathrm{D}}\end{array}$ & $\begin{array}{l}\Delta P_{\text {ave }} \\
{[\mathrm{kPa}]}\end{array}$ \\
\hline \multirow{3}{*}{5} & Equilibrium & 0.21 & - & 1.4 \\
\hline & Quasi-equilibrium & 0.19 & - & 1.4 \\
\hline & Non-equilibrium & 0.23 & 97 & 1.2 \\
\hline \multirow{3}{*}{10} & Equilibrium & 0.21 & - & 1.4 \\
\hline & Quasi-equilibrium & 0.19 & - & 1.4 \\
\hline & Non-equilibrium & 0.21 & 144 & 1.4 \\
\hline \multirow{3}{*}{19.3} & Equilibrium & 0.21 & - & 1.8 \\
\hline & Quasi-equilibrium & 0.20 & - & 1.9 \\
\hline & Non-equilibrium & 0.23 & 82 & 1.6 \\
\hline \multirow{3}{*}{26.3} & Equilibrium & 0.21 & - & 1.7 \\
\hline & Quasi-equilibrium & 0.21 & - & 1.9 \\
\hline & Non-equilibrium & 0.22 & 120 & 1.5 \\
\hline
\end{tabular}


significant difference between the methane concentrations determined by using these two $\mathrm{BCs}$ at the $\mathrm{CH}_{4}$-heavy oil interface.

Moreover, at each diffusion time, when the minimum $\Delta P_{\text {ave }}$ values for the three $\mathrm{BCs}$ are compared, the non-equilibrium $\mathrm{BC}$ gives the smallest minimum $\Delta P_{\text {ave }}$ value. However, the differences among the minimized objective functions are very small. It is also found that the mass-transfer Biot number $k_{\mathrm{D}}$ increases with the diffusion time. This means that the resistance at the solvent-heavy oil interface becomes less important at large times, i.e., the non-equilibrium $\mathrm{BC}$ approaches the equilibrium $\mathrm{BC}$. Therefore, methane-heavy oil system initially follows the non-equilibrium $\mathrm{BC}$ and later on the equilibrium or quasi-equilibrium $\mathrm{BC}$ once the interface is saturated with the solvent. As methane belongs to the same family of hydrocarbons as heavy oil, the hydrocarbon solvent-heavy oil interface is saturated more readily than any non-hydrocarbon solventheavy oil interface, such as carbon dioxide-heavy oil interface. In general, the determination of diffusion coefficient of $\mathrm{CH}_{4}$-heavy oil system is insensitive to the boundary condition applied at the solvent-heavy oil interface at large times (Tharanivasan et al., 2004b).

At the diffusion time of $t=5$ days, the calculated pressures for the three different BCs are compared with the measured pressures in Fig. 5.14a for Run-1 and Fig 5.14b for Run-2. It can be seen from these two figures that the differences between the calculated and measured pressures are slightly larger at the beginning for both the equilibrium and quasi-equilibrium $\mathrm{BCs}$, in comparison with those for the non-equilibrium $\mathrm{BC}$. This means that methane-heavy oil interface cannot be saturated instantaneously. Thus, the equilibrium and quasi-equilibrium $\mathrm{BCs}$ cannot be applied at the very beginning. If 


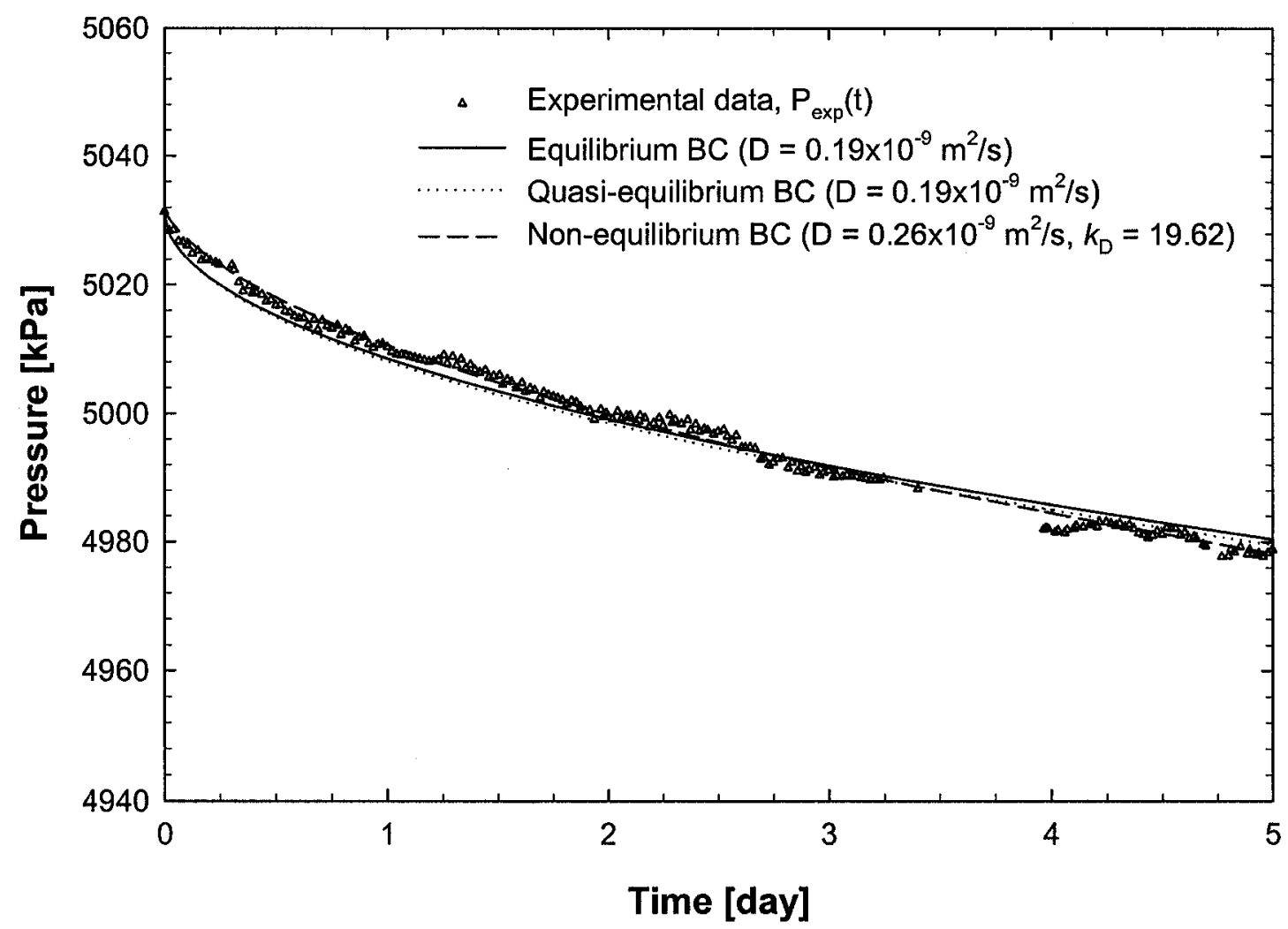

Figure 5.14a. History matching of the calculated pressures under three different BCs with the experimental data ( $t=5$ days) for $\mathrm{CH}_{4}$-heavy oil system (Run-1) at $T=23.9^{\circ} \mathrm{C}$. 


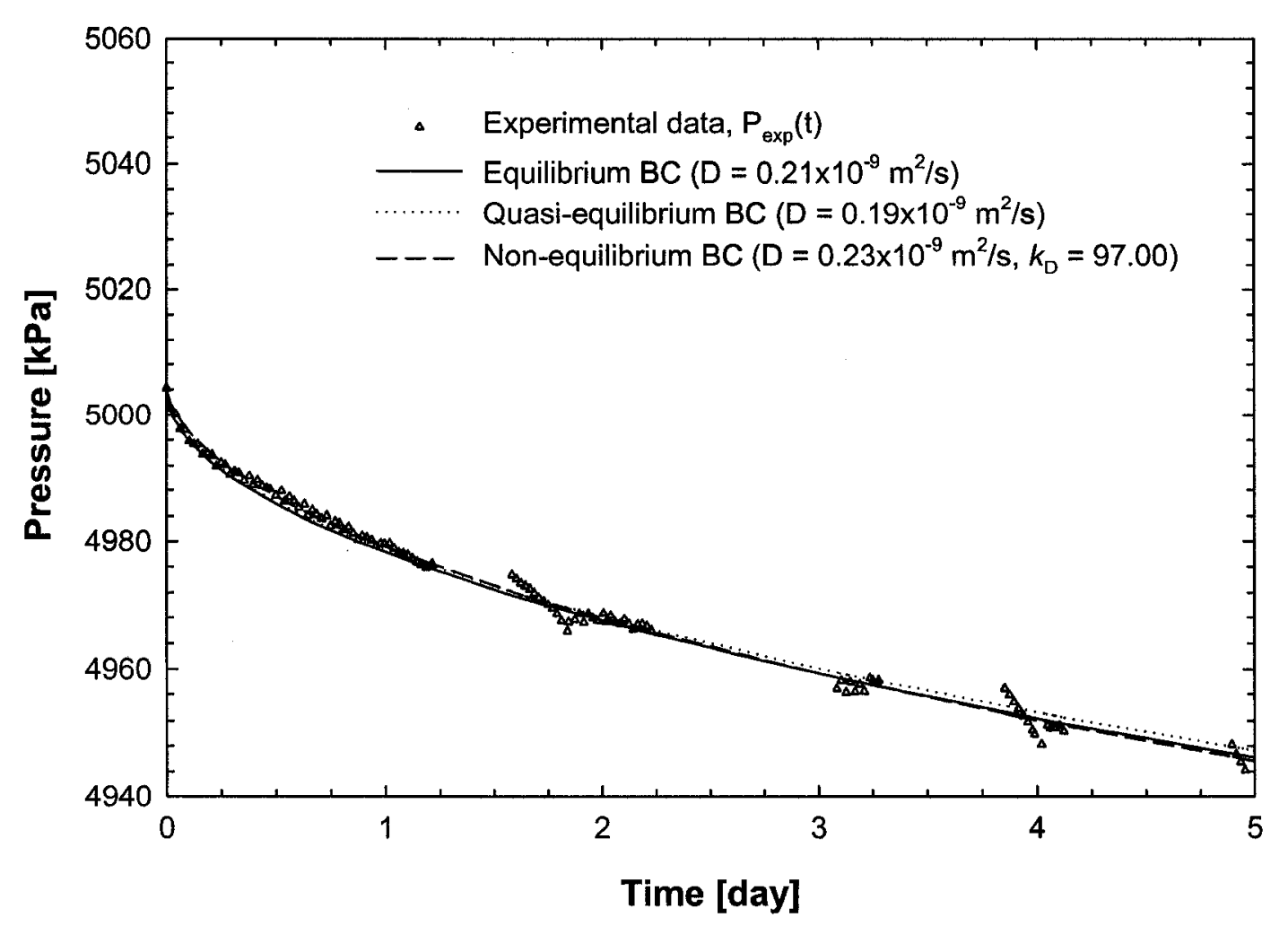

Figure 5.14b. History matching of the calculated pressures under three different BCs with the experimental data ( $t=5$ days) for $\mathrm{CH}_{4}$-heavy oil system (Run-2) at $T=23.9^{\circ} \mathrm{C}$. 
the interfacial resistance is taken into account at early stages of diffusion (i.e., the nonequilibrium $\mathrm{BC}$ is used), the diffusivity and mass-transfer Biot number for $\mathrm{CH}_{4}$-heavy oil system are found to be $0.26 \times 10^{-9} \mathrm{~m}^{2} / \mathrm{s}$ and 19.62 for Run- 1 , and $0.23 \times 10^{-9} \mathrm{~m}^{2} / \mathrm{s}$ and 97.00 for Run-2, respectively.

The dimensionless solvent concentration profiles in heavy oil at various times, $t=$ $0.5,1.0,2.0,5.0,10.0,15.0,20.0,23.8$ and 50.0 days, under the non-equilibrium $\mathrm{BC}$ are plotted in Fig. 5.15a for $\mathrm{CH}_{4}$-heavy oil system (Run-1). Similarly, Fig. 5.15b shows the dimensionless solvent concentration profiles plotted at various times, $t=0.5,1.0,2.0,5.0$, $10.0,15.0,20.0,26.3,50.0$ and 75.0 days, for Run-2 experiment. These two figures clearly show that methane diffuses to the bottom of the diffusion cell in about 2 days for Run-1 and in about 4 days for Run-2. This difference is due to different amounts of heavy oil used in these two experiments. In Run-1 experiment, the volume percentage of heavy oil in the diffusion cell is $16.4 \%$, whereas it is about $24.4 \%$ in Run-2 experiment. Certainly, during Run-2 experiment methane takes longer time to reach the bottom of the diffusion cell. In addition, it is noted from Figs. 5.15a and 5.15b that even up to 50.0 days, the dimensionless solvent concentration in heavy oil at the bottom of the diffusion cell reaches only $93 \%$ for Run- 1 , and $73 \%$ for Run- 2 . Given the fact that the diffusion process of $\mathrm{CH}_{4}$-heavy oil system takes an extremely long time to reach the equilibrium state, in this study, the equilibrium pressure is determined from the measured solubility data at the same temperature. 


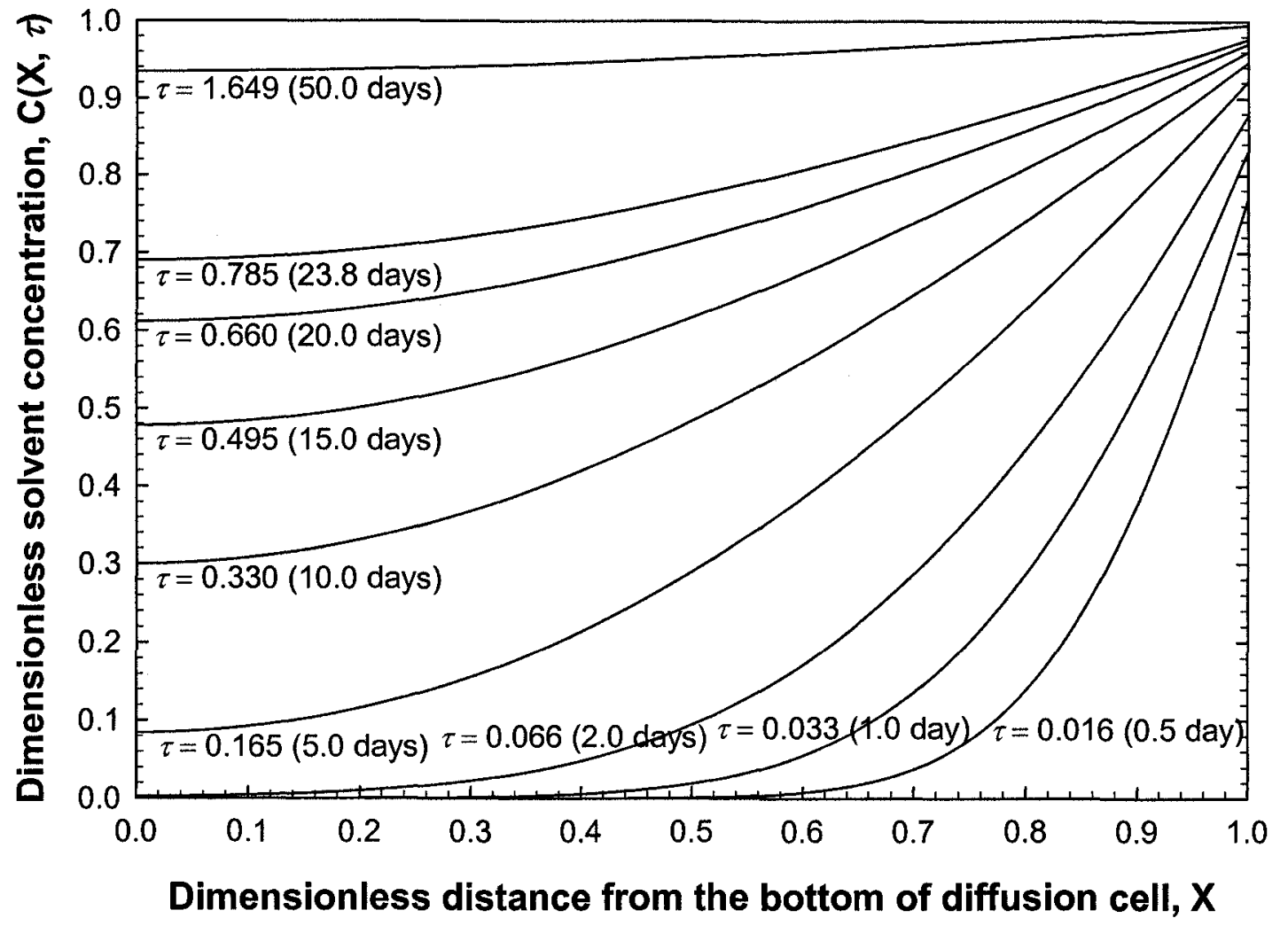

Figure 5.15a. Dimensionless solvent concentration profiles for $\mathrm{CH}_{4}$-heavy oil system (Run-1) at different times under the non-equilibrium boundary condition $\left(D=0.26 \times 10^{-9}\right.$ $\mathrm{m}^{2} / \mathrm{s}$ and $k_{\mathrm{D}}=19.62$ ). 


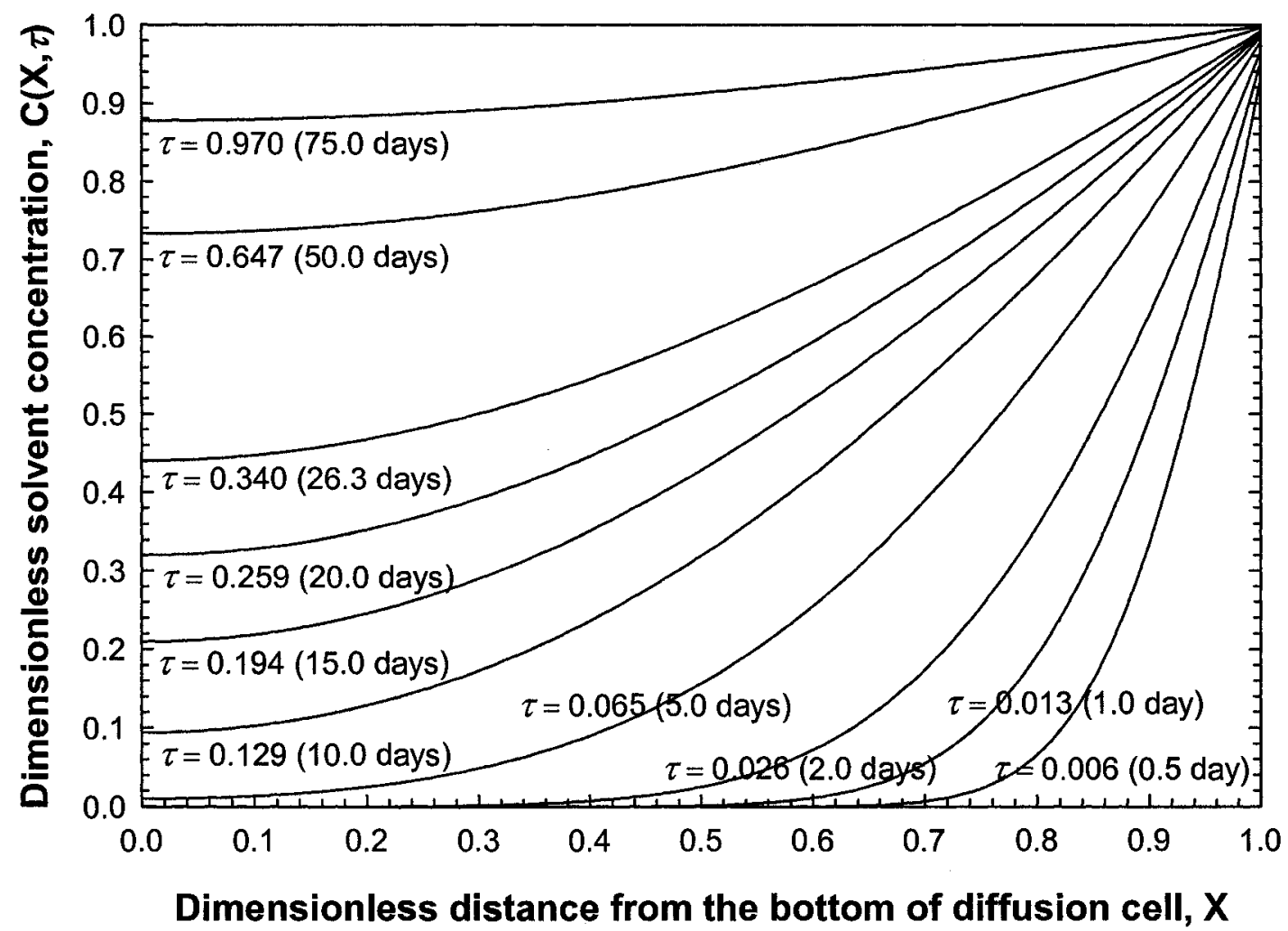

Figure 5.15b. Dimensionless solvent concentration profiles for $\mathrm{CH}_{4}$-heavy oil system (Run-2) at different times under the non-equilibrium boundary condition $\left(D=0.23 \times 10^{-9}\right.$ $\mathrm{m}^{2} / \mathrm{s}$ and $k_{\mathrm{D}}=97.00$ ). 


\subsubsection{Propane-heavy oil system}

The measured pressure decay data for both the experimental runs conducted for propane-heavy oil system are plotted in Figs. 5.16a and 5.16b, respectively. These two plots include the best-fit curves of the experimental data at different diffusion times, i.e., $t$ $=5,10,20$ and 23.5 days for Run-1, and $t=5,10$ and 18 days for Run-2. Equation [3.12] along with the known equilibrium pressure, $P_{\text {eq }}$, determined the previous section 5.2 .1 is used to find the best-fit curve for each diffusion time. The non-linear regression yields excellent correlation coefficients $\left(R^{2} \geq 0.996\right)$ for all the diffusion times of the two experimental runs for propane-heavy oil system. This indicates that the assumed expression for pressure as a function of time in Eq. [3.12] is also an excellent approximation for propane-heavy oil system. The values of the parameters, $m_{1}, m_{2}, k_{1}$ and $k_{2}$ defined in Eq. [3.12], are found for each diffusion time and given in the regressed equation, which is also included in Fig. 5.16a for Run-1 and in Fig. 5.16b for Run-2.

The diffusion coefficients of propane in heavy oil at different diffusion times for each of the three different BCs are given in Table 5.6a for Run-1, and Table 5.6b for Run-2, respectively. When the minimum objective functions $\Delta P_{\text {ave }}$ given in both these tables for three different $\mathrm{BCs}$ at each diffusion time are compared, the quasi-equilibrium $\mathrm{BC}$ always gives the best history matching of the calculated pressures with the measured data. Therefore, the quasi-equilibrium $\mathrm{BC}$ is found to be more applicable throughout the diffusion process for propane-heavy oil system (Tharanivasan et al., 2004c). Physically, this reveals that the heavy oil at the interface is saturated instantaneously with propane at the existing pressure in the solvent phase. Hence, the solvent concentration at propaneheavy oil interface decreases as the diffusion proceeds. On the other hand, when the 


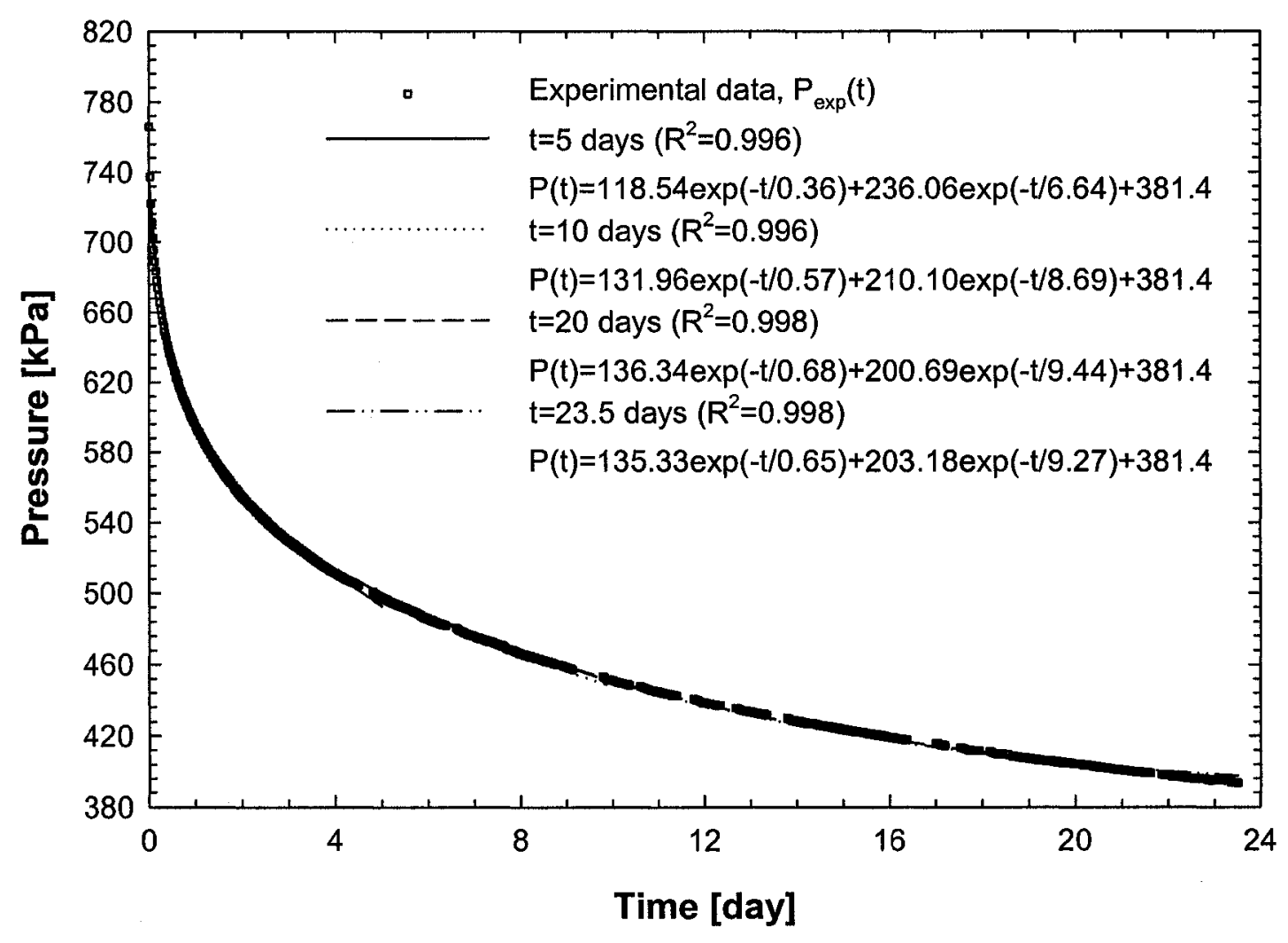

Figure 5.16a. Measured pressure decay data and the best-fit curves at different diffusion times for $\mathrm{C}_{3} \mathrm{H}_{8}$-heavy oil system (Run-1) at $T=23.9^{\circ} \mathrm{C}$. 


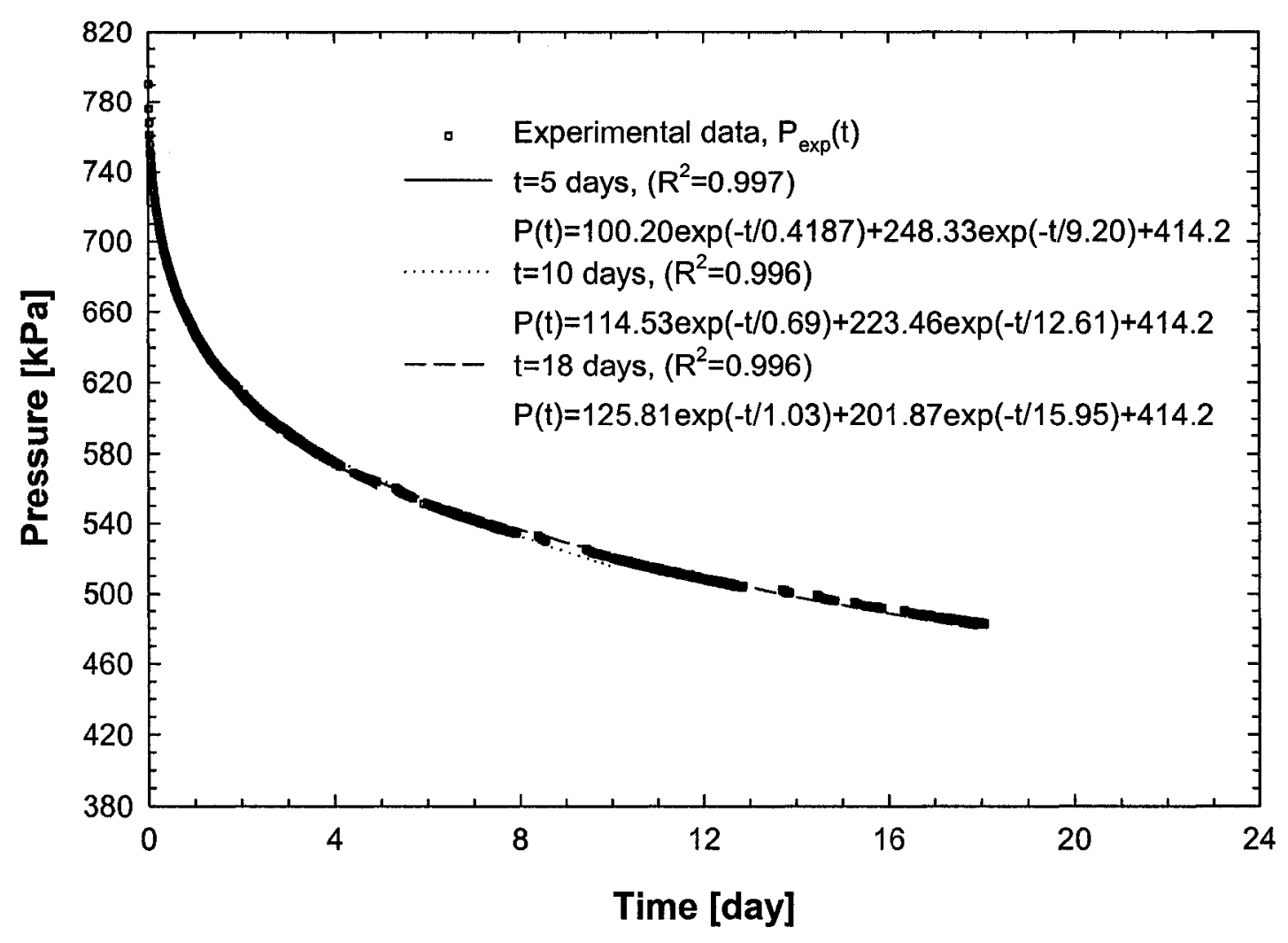

Figure 5.16b. Measured pressure decay data and the best-fit curves at different diffusion times for $\mathrm{C}_{3} \mathrm{H}_{8}$-heavy oil system (Run-2) at $T=23.9^{\circ} \mathrm{C}$. 
Table 5.6a. Comparison of diffusion coefficients at different diffusion times for $\mathrm{C}_{3} \mathrm{H}_{8}$ heavy oil system (Run-1) at $T=23.9^{\circ} \mathrm{C}$.

\begin{tabular}{|c|c|c|c|c|}
\hline $\begin{array}{c}\text { Diffusion time } \\
\text { [day }]\end{array}$ & Boundary condition & $\begin{array}{c}\text { Diffusivity } \\
D\left[10^{-9} \mathrm{~m}^{2} / \mathrm{s}\right]\end{array}$ & $\begin{array}{c}\text { Biot number } \\
k_{\mathrm{D}}\end{array}$ & $\begin{array}{c}\Delta P_{\text {ave }} \\
{[\mathrm{kPa}]}\end{array}$ \\
\hline \multirow{4}{*}{5} & Equilibrium & 2.53 & - & 23.6 \\
\cline { 2 - 5 } & Quasi-equilibrium & 1.10 & - & 16.1 \\
\cline { 2 - 5 } & Non-equilibrium & 2.55 & $>500$ & $<23.7$ \\
\hline \multirow{3}{*}{10} & Equilibrium & 2.01 & - & 28.8 \\
\cline { 2 - 5 } & Quasi-equilibrium & 0.92 & - & 22.0 \\
\cline { 2 - 6 } & Non-equilibrium & 2.02 & $>500$ & $<28.9$ \\
\hline \multirow{4}{*}{20} & Equilibrium & 1.78 & - & 27.7 \\
\cline { 2 - 6 } & Quasi-equilibrium & 0.80 & - & 23.4 \\
\cline { 2 - 6 } & Non-equilibrium & 1.79 & $>450$ & $<27.8$ \\
\hline \multirow{2}{*}{23.5} & Equilibrium & 1.76 & - & 25.8 \\
\cline { 2 - 6 } & Quasi-equilibrium & 0.79 & - & 22.0 \\
\cline { 2 - 6 } & Non-equilibrium & 1.78 & $>300$ & $<26.0$ \\
\hline
\end{tabular}


Table 5.6b. Comparison of diffusion coefficients at different diffusion times for $\mathrm{C}_{3} \mathrm{H}_{8}$ heavy oil system (Run-2) at $T=23.9^{\circ} \mathrm{C}$.

\begin{tabular}{|c|c|c|c|c|}
\hline $\begin{array}{c}\text { Diffusion time } \\
{[\text { day }]}\end{array}$ & Boundary condition & $\begin{array}{c}\text { Diffusivity, } \\
D\left[10^{-9} \mathrm{~m}^{2} / \mathrm{s}\right]\end{array}$ & $\begin{array}{c}\text { Biot number } \\
k_{\mathrm{D}}\end{array}$ & $\begin{array}{c}\Delta P_{\text {ave }} \\
{[\mathrm{kPa}]}\end{array}$ \\
\hline \multirow{5}{*}{5} & Equilibrium & 1.63 & - & 19.8 \\
\cline { 2 - 5 } & Quasi-equilibrium & 0.71 & - & 13.9 \\
\cline { 2 - 5 } & Non-equilibrium & 1.64 & $>400$ & $<20.0$ \\
\hline \multirow{3}{*}{10} & Equilibrium & 1.28 & - & 26.6 \\
\cline { 2 - 6 } & Quasi-equilibrium & 0.59 & - & 20.0 \\
\cline { 2 - 6 } & Non-equilibrium & 1.29 & $>350$ & $<26.8$ \\
\hline \multirow{3}{*}{18.07} & Equilibrium & 1.02 & - & 31.1 \\
\cline { 2 - 6 } & Quasi-equilibrium & 0.49 & - & 25.5 \\
\cline { 2 - 6 } & Non-equilibrium & 1.03 & $>350$ & $<31.3$ \\
\hline
\end{tabular}


equilibrium and non-equilibrium BCs are applied at the interface, the history matching of the calculated pressures with the measured pressures results in similar $D$ and $\Delta P_{\text {ave }}$ values. This is because the non-equilibrium $\mathrm{BC}$ approaches the equilibrium $\mathrm{BC}$ if a sufficiently large value of $k_{\mathrm{D}}\left(k_{\mathrm{D}}=500\right.$ for Run-1 and $k_{\mathrm{D}}=400$ for Run-2) is used. Certainly, this also means that propane-heavy oil interface does not offer any resistance to the mass transfer of propane in heavy oil.

At the diffusion time of $t=5$ days, the calculated pressures for the three different BCs are compared with the measured pressures in Fig. 5.17a for Run-1, and Fig. 5.17b for Run-2. These two figures clearly show that the pressures calculated by applying the quasi-equilibrium $\mathrm{BC}$ at the solvent-heavy oil interface gives relatively better fit to the measured pressure decay data, in comparison with the other two BCs. In addition, it is found that the calculated pressures found by applying equilibrium and non-equilibrium $\mathrm{BC}$ are almost the same. When the quasi-equilibrium $\mathrm{BC}$ is applied, the diffusivity of propane in heavy oil is found to be $1.10 \times 10^{-9} \mathrm{~m}^{2} / \mathrm{s}$ for Run- 1 , and $0.71 \times 10^{-9} \mathrm{~m}^{2} / \mathrm{s}$ for Run2. These two figures also show that, in general, the calculated pressures are larger at small times ( $t \leq 2.7$ days for Run- 1 and $t \leq 2.8$ days for Run-2) and smaller at large times ( $t>2.7$ days for Run-1 and $t>2.8$ days for Run-2). Accordingly, the predicted mass transfer rate of propane in heavy oil is underestimated at small times and overestimated at large times, respectively. This may be caused by the assumption of constant diffusion coefficient throughout the diffusion process. Figures $5.17 \mathrm{a}$ and $5.17 \mathrm{~b}$ also indicate that propane diffusivity in heavy oil is larger at small times and smaller at large times. Physically, it is speculated that the accelerated mass transfer of propane in heavy oil occurs at the initial stages due to the convective flow in the heavy oil phase, which is 


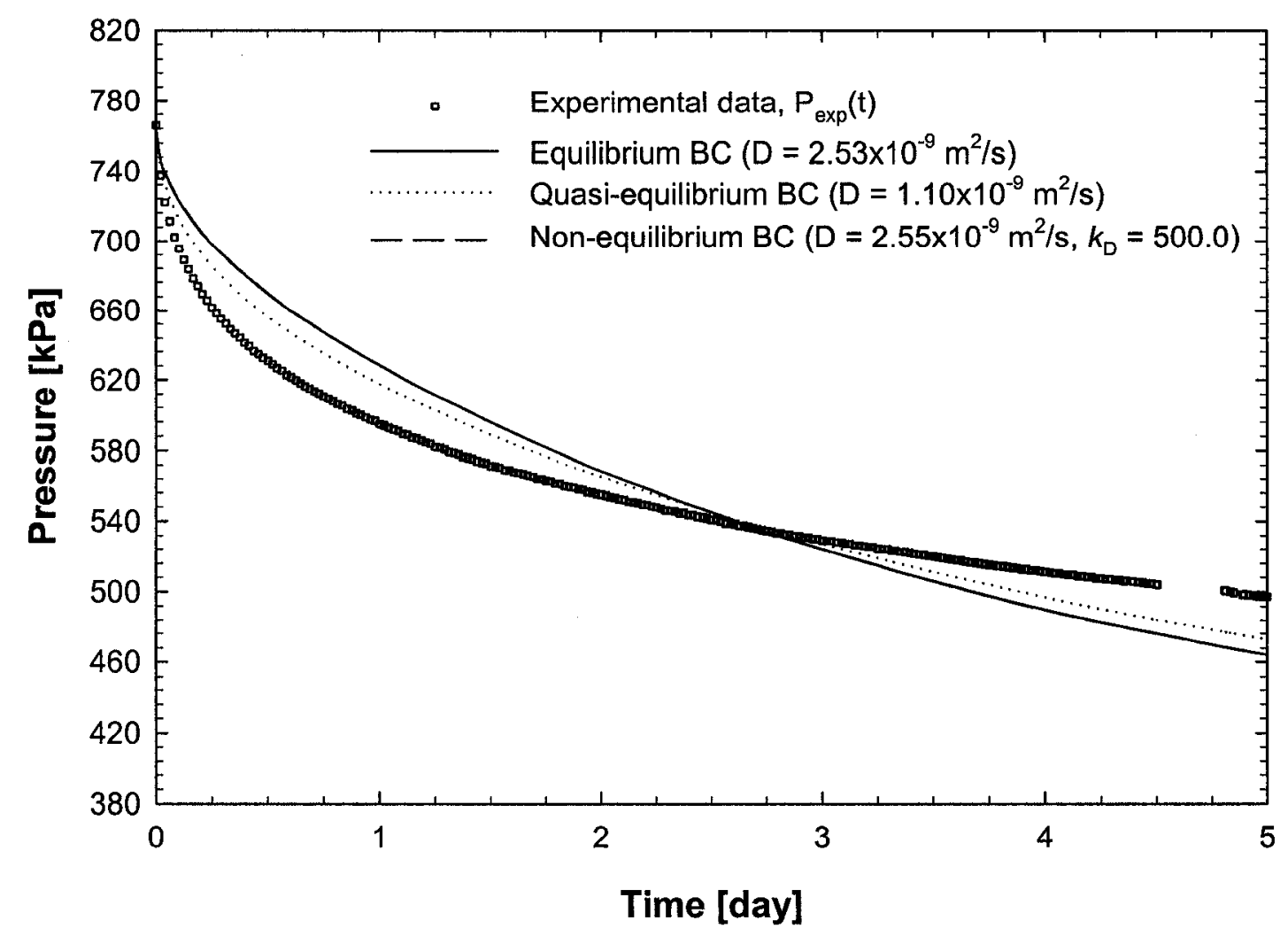

Figure 5.17a. History matching of the calculated pressures under three different BCs with the experimental data ( $t=5$ days) for $\mathrm{C}_{3} \mathrm{H}_{8}$-heavy oil system (Run-1) at $T=23.9^{\circ} \mathrm{C}$. 


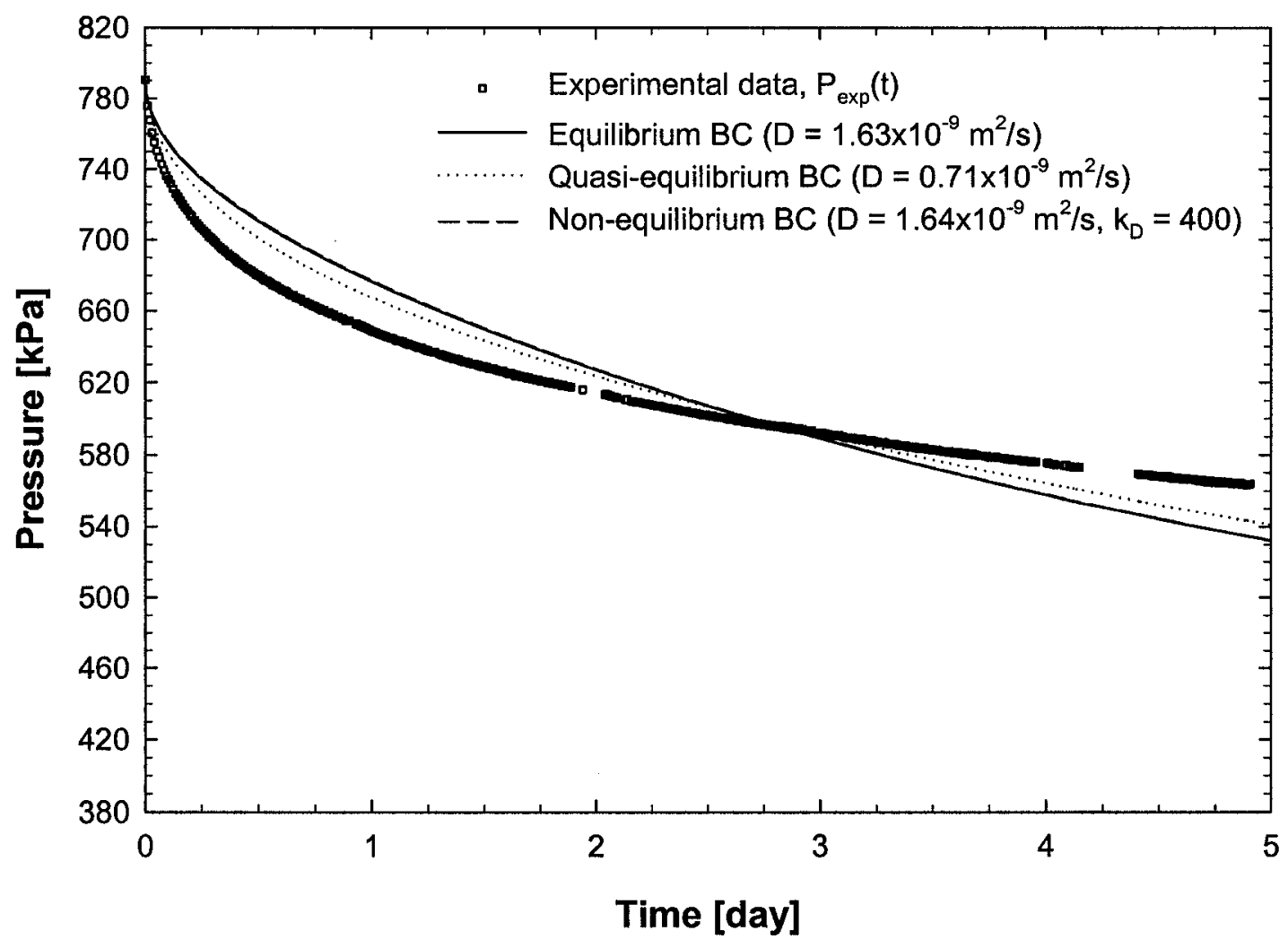

Figure 5.17b. History matching of the calculated pressures under three different BCs with the experimental data $\left(t=5\right.$ days) for $\mathrm{C}_{3} \mathrm{H}_{8}$-heavy oil system (Run-2) at $T=23.9^{\circ} \mathrm{C}$. 
caused by the asphaltene precipitation. Frauenfeld et al. (2002) found that there is possibility of asphaltene precipitation above $P=600 \mathrm{kPa}$ at $T=15^{\circ} \mathrm{C}$ for propane-heavy oil system. In this study, the diffusion test of propane in the heavy oil sample starts at $P_{\text {in }}=765.9 \mathrm{kPa}$ and ends at $P_{\mathrm{t}}=393.3 \mathrm{kPa}$ for Run- 1 . For Run-2, $P_{\text {in }}=790.4 \mathrm{kPa}$ and ends at $P_{\mathrm{t}}=482.0 \mathrm{kPa}$. Therefore, weak asphaltene precipitation is expected to take place.

With the determined diffusivity $\left(D=1.10 \times 10^{-9} \mathrm{~m}^{2} / \mathrm{s}\right)$, the dimensionless solvent concentration profiles in heavy oil at various times, $t=0.25,0.5,1.0,2.0,5.0,10.0,15.0$ and 23.5 days, are predicted by using the quasi-equilibrium $\mathrm{BC}$ and plotted in Fig. 5.18a for $\mathrm{C}_{3} \mathrm{H}_{8}$-heavy oil system (Run-1). Similar plot for Run-2 with the determined diffusivity $\left(D=0.71 \times 10^{-9} \mathrm{~m}^{2} / \mathrm{s}\right)$, the dimensionless solvent concentration profiles in heavy oil at various times, $t=0.25,0.5,1.0,2.0,5.0,10.0,15.0$ and 18.0 days, are presented in Fig. 5.18b. These two figures clearly show that the solvent concentration at the interface decreases as the diffusion proceeds. In addition, it is noted that propane reaches the bottom of the diffusion cell within approximately one day. It saturates the heavy oil much faster than the other two solvents, i.e., carbon dioxide as shown in Figs. 5.12a-b and methane as shown in Figs. 5.15a-b. This is attributed to its larger diffusivity and higher interface solvent concentration.

\subsection{Summary}

The diffusion coefficients of carbon dioxide, methane and propane in the given heavy oil are determined from this thesis study by using the pressure decay method. The complete summary of the obtained results is tabulated in Table 5.7. The following is the list of some major achievements made in the course of this thesis study. A state-of-the-art 


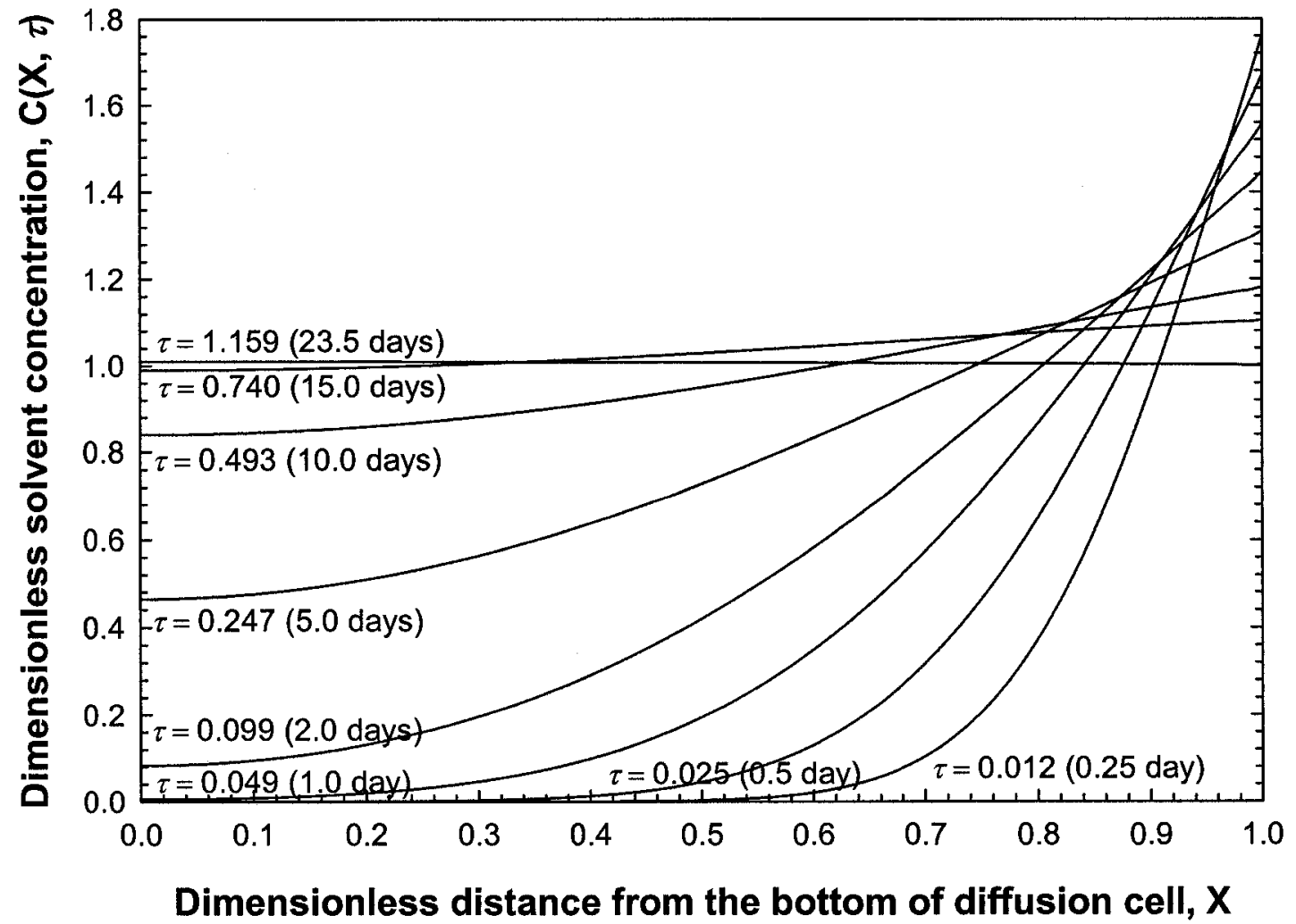

Figure 5.18a. Dimensionless solvent concentration profiles for $\mathrm{C}_{3} \mathrm{H}_{8}$-heavy oil system (Run-1) at different times under the quasi-equilibrium boundary condition $\left(D=1.10 \times 10^{-9} \mathrm{~m}^{2} / \mathrm{s}\right)$. 


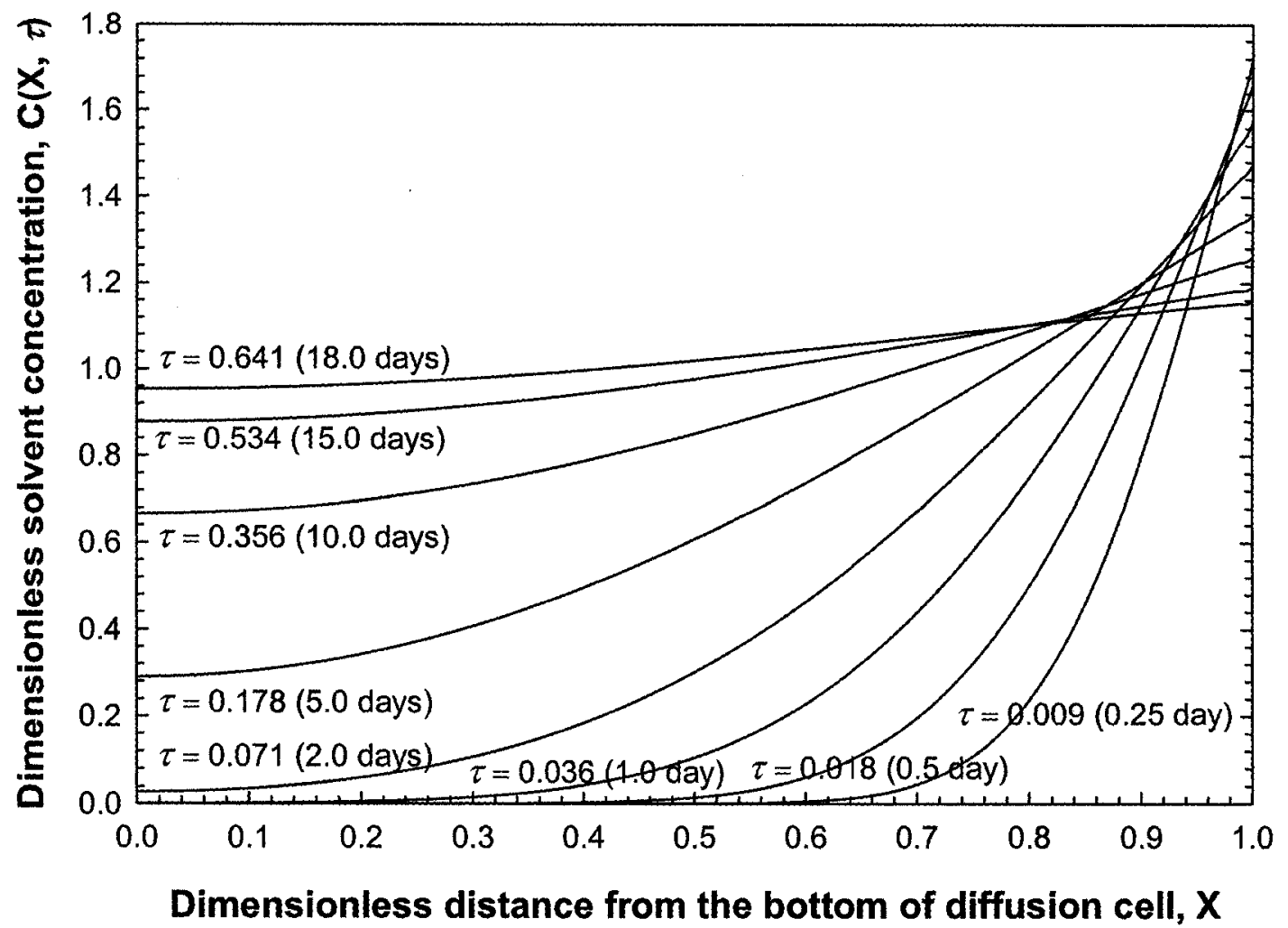

Figure 5.18b. Dimensionless solvent concentration profiles for $\mathrm{C}_{3} \mathrm{H}_{8}$-heavy oil system (Run-2) at different times under the quasi-equilibrium boundary condition $\left(D=0.71 \times 10^{-9} \mathrm{~m}^{2} / \mathrm{s}\right)$. 
Table 5.7. Summary of experimental results for three solvent-heavy oil systems at $T=23.9^{\circ} \mathrm{C}$.

\begin{tabular}{|c|c|c|c|c|c|c|}
\hline \multirow{3}{*}{ Parameters } & \multicolumn{6}{|c|}{ Solvent-heavy oil system } \\
\hline & \multicolumn{2}{|c|}{$\mathrm{CO}_{2}$-heavy oil } & \multicolumn{2}{|c|}{$\mathrm{CH}_{4}$-heavy oil } & \multicolumn{2}{|c|}{$\mathrm{C}_{3} \mathrm{H}_{8}$-heavy oil } \\
\hline & Run-1 & Run-2 & Run-1 & Run-2 & Run-1 & Run-2 \\
\hline Initial pressure $[\mathrm{kPa}]$ & $4,172.5$ & $4,210.0$ & $5,031.3$ & $5,004.2$ & 765.9 & 790.4 \\
\hline Termination pressure $[\mathrm{kPa}]$ & $3,530.0$ & $3,815.0$ & $4,918.0$ & $4,872.0$ & 393.3 & 482.0 \\
\hline Equilibrium pressure $[\mathrm{kPa}]$ & $3,480.7$ & $3,558.8$ & $4,901.1$ & $4,793.0$ & 381.4 & 414.2 \\
\hline $\begin{array}{l}\text { Solubility at the equilibrium pressure } \\
\text { [g solvent } / 100 \mathrm{~g} \text { heavy oil] }\end{array}$ & 5.852 & 6.014 & 0.526 & 0.514 & 2.316 & 2.490 \\
\hline Diffusion coefficient $\left[10^{-9} \mathrm{~m}^{2} / \mathrm{s}\right]$ & 0.94 & 0.92 & 0.26 & 0.23 & 1.10 & 0.71 \\
\hline Mass-transfer Biot number & 19.20 & 19.90 & 19.62 & 97.00 & - & - \\
\hline Recommended boundary condition & Non-equi & ibrium & $\begin{array}{l}\text { Any on } \\
\text { three BC}\end{array}$ & of the & Quasi-e & ilibrium \\
\hline
\end{tabular}


experimental setup for applying the pressure decay method is established. It is also examined which boundary condition should be applied at the solvent-heavy oil interface. In addition, the diffusion coefficient is determined by using the pressure decay data measured in a short duration. Finally, it is worthwhile to emphasize that the diffusion coefficient of propane in heavy oil is measured for the first time.

The experimental setup of the pressure decay method is established at the Petroleum Technology Research Centre (PTRC) to accurately measure the decaying pressure during the diffusion process at constant temperature. The major components of this setup include high-pressure high-temperature diffusion cell, high-precision pressure transducer, automatic data acquisition system for both pressure and temperature, and constant temperature control system. Secondly, the proper boundary condition at the solvent-heavy oil interface is examined by using both the pressure decay data available in the literature (Zhang et al., 2000) and those obtained by using the established experimental setup during this thesis study. The recommended BC at the solvent-heavy oil interface for each of the following three solvent-heavy oil systems, $\mathrm{CO}_{2}$-heavy oil, $\mathrm{CH}_{4}$-heavy oil and $\mathrm{C}_{3} \mathrm{H}_{8}$-heavy oil systems, is given in Table 5.7. Thirdly, a new strategy is adopted to determine the equilibrium pressure from the measured solubility data for the same solvent-heavy oil system. Thus with the pressure decay data measured for a short period, one can determine the diffusion coefficient by using the pressure decay method. Finally, the diffusion coefficient of propane in heavy oil is measured for the first time. In the literature, only an empirical correlation is available for relating the diffusivity of propane to the viscosity of propane-bitumen mixture (Das and Butler, 1998). 


\section{Chapter 6 CONCLUSIONS AND RECOMMENDATIONS}

\subsection{Conclusions}

In this thesis study, the diffusion coefficients of a non-hydrocarbon solvent, i.e., carbon dioxide, and two hydrocarbon solvents, i.e., methane and propane, in the given heavy oil sample are determined under reservoir conditions by using the so-called pressure decay method. The major conclusions that can be made from this study are listed as follows.

1. A state-of-the-art experimental setup for applying the pressure decay method is established at the Petroleum Technology Research Centre (PTRC), University of Regina, to accurately determine the diffusion coefficient of a gas solvent in given heavy oil at constant temperature.

2. A novel idea is proposed and has been successfully utilized to determine the diffusion coefficient of a specific solvent in heavy oil by minimizing the average pressure difference between the calculated and measured pressure decay data. The numerical optimization is performed by using the simultaneous search method.

3. For the given heavy oil sample, among the three solvents tested at constant temperature, methane has the lowest diffusivities even at high pressures, in comparison with carbon dioxide and propane. The diffusivities of carbon dioxide and propane in heavy oil are close to each other.

4. Three different boundary conditions that can be applied at the solvent-heavy oil interface are thoroughly examined in the determination of diffusion coefficient. For carbon dioxide-heavy oil system, it is found that the non-equilibrium $\mathrm{BC}$ is most 
applicable at the solvent-heavy oil interface. For methane-heavy oil system, the determination of diffusivity is insensitive to the boundary condition applied at the interface. The diffusion process of propane in heavy oil is best described by applying the quasi-equilibrium $\mathrm{BC}$ at the interface. Three FORTRAN programs are developed for determining the diffusion coefficient under each of the three different boundary conditions.

5. A new strategy is adopted to determine the equilibrium pressure of each pressure decay experiment by using the measured solubility of the solvent in heavy oil rather than by measuring the equilibrium pressure at the end of diffusion experiment. A FORTRAN program is developed to determine the equilibrium pressure from the measured solubility data for each solvent-heavy oil system.

6. With the determined equilibrium pressure, the diffusion coefficient of each solvent in heavy oil can be determined by measuring the pressure decay data for a short duration. Thus, in the diffusion experiment, there is no need to measure the pressure decay for an extremely long time until the heavy oil is completely saturated.

7. The solubility measurements are carried out by using a newly constructed vapourliquid equilibrium (VLE) apparatus, which is established at the Petroleum Technology Research Centre (PTRC), University of Regina, during this thesis study.

8. Measured solubility data of carbon dioxide in heavy oil are approximately one order higher than those of methane in heavy oil. Therefore, more carbon dioxide can diffuse into heavy oil than methane under the same pressure and temperature conditions. On the other hand, the solubility of propane in heavy oil is high even at a low pressure. 
9. The solubility vs. pressure relationship is obtained in a certain pressure range for each solvent-heavy oil system. It is found that there is excellent linear relationship for $\mathrm{CO}_{2}$-heavy oil and $\mathrm{CH}_{4}$-heavy oil systems, whereas good linearity exists for $\mathrm{C}_{3} \mathrm{H}_{8}$ heavy oil system.

10. Theoretically, the pressure decay data are calculated by using the conservation of mass and an equation of state for a real gas. In this study, the compressibility factor used in the equation of state is calculated by using the Lee-Kesler correlation, instead of assuming it to be constant throughout the diffusion process. This increases the computation accuracy in calculating the pressure decay data.

\subsection{Recommendations}

This study is aimed at facilitating the field design of VAPEX and other solventbased recovery process by improving the existing pressure decay method in the determination of diffusion coefficient of solvent in heavy oil. In this regard, the following recommendations are made for future research.

1. The pressure and temperature effects on the determined solvent diffusion coefficient in heavy oil can be studied by conducting the pressure decay experiment at various pressures and temperatures.

2. The developed diffusion model and the experimental setup for pressure decay method could be used to determine the diffusion coefficient of other solvents of interest in heavy oil, e.g., non-hydrocarbon solvents, such as carbon monoxide and nitrogen, and hydrocarbon solvents, such as ethane and butane. 
3. The pressure decay method can be extended to multi-component solvent diffusion in heavy oil. In this case, the diffusion model should be modified to determine an effective diffusion coefficient.

4. As an important improvement to the pressure decay method, the oil swelling effect during the diffusion process may be observed and measured by using a see-through windowed diffusion cell and a computer-aided digital image acquisition and processing system. Thus the oil swelling effect can be incorporated into the present diffusion model.

5. The solubility measurement of each solvent in given heavy oil can be made for a wide pressure range. Therefore, the linear assumption made in obtaining the semianalytical solution for the quasi-equilibrium $\mathrm{BC}$ can be thoroughly examined. 


\section{REFERENCES}

Akai, T.J., Applied Numerical Methods for Engineers, John Wiley \& Sons, Inc., New York, 1994.

Akgerman, A., and Gainer, J.L., Diffusion of Gases in Liquids, Ind. Eng. Chem. Fundam., 11 (3), 373-379, 1972.

Bird, R.B., Stewart, W.E., and Lightfoot, E.N., Transport Phenomena, Second Edition, John Wiley \& Sons, Inc., New York, 2002.

Boustani, A., and Maini, B.B., The Role of Diffusion and Convective Dispersion in Vapour Extraction Process, J. Can. Pet. Tech., 40 (4), 68-77, 2001.

Butler, R.M., and Mokrys, I.J., A New Process (VAPEX) for Recovering Heavy Oils Using Hot Water and Hydrocarbon Vapour, J. Can. Pet. Tech., 30 (1), 97-106, 1991. Butler, R.M., and Yee, C.T., Progress in the In Situ Recovery of Heavy Oils and Bitumen, J. Can. Pet. Tech., 41 (1), 31-40, 2002.

Carslaw, H.S., and Jaeger, J.C., Conduction of Heat in Solids, Oxford University Press, London, 1959.

Civan, F., and Rasmussen, M.L., Accurate Measurement of Gas Diffusivity in Oil and Brine Under Reservoir Conditions, Paper SPE 67319, SPE Production and Operations Symposium, Oklahoma City, OK, 2001.

Civan, F., and Rasmussen, M.L., Improved Measurement of Gas Diffusivity for Miscible Gas Flooding Under Non-equilibrium vs. Equilibrium conditions, Paper SPE 75135, SPE/DOE Improved Oil Recovery Symposium, Tulsa, OK, 2002.

Civan, F., and Rasmussen, M.L., Analysis and Interpretation of Gas Diffusion in Quiescent Reservoir, Drilling and Completion Fluids: Equilibrium vs. Non- 
equilibrium Models, Paper SPE 84072, SPE Annual Technical Conference and Exhibition, Denver, CO, 2003.

Crank, J., The Mathematics of Diffusion, Second Edition, Clarendon Press, Oxford, 1975. Cussler, E.L., Diffusion Mass Transfer in Fluid Systems, Second Edition, Cambridge University Press, Cambridge, 1997.

Cuthiell, D., McCarthy, C., Frauengeld, T., Scameron, S., and Kissel, G., Investigation of the VAPEX Process Using CT Scanning and Numerical Simulation, J. Can. Pet. Tech., 42 (2), 41-49, 2003.

Dake, L.P., Fundamentals of Reservoir Engineering, Elsevier, New York, 1978.

Das, S.K., Vapex: An Efficient Process for the Recovery of Heavy Oil and Bitumen, SPE $J ., 3(3), 232-237,1998$.

Das, S.K., and Butler, R.M., Diffusion Coefficients of Propane and Butane in Peace River Bitumen, Can. J. Chem. Eng., 74 (6), 985-991, 1996.

Das, S.K., and Butler R.M., Mechanism of the Vapor Extraction Process for Heavy Oil and Bitumen, J. Pet. Sci. \& Eng., 21 (1-2), 43-59, 1998.

Denoyelle, L., and Bardon, C., Diffusivity of Carbon Dioxide in Reservoir Fluids, Paper 114, Annual Meeting of Canadian Institute of Mining, Metallurgy and Petroleum, Ottawa, ON, 1984.

Duda, J.L., and Vrentas, J.S., Diffusion in Polymer Solutions - An Overview, AIChE Annual Meeting, Los Angeles, CA, 1982.

Edmister, W.C., and Lee, B.I., Applied Hydrocarbon Thermodynamics, Vol. 1, Second Edition, Gulf Publishing Company, Houston, TX, 1984. 
Frauenfeld, T.W.J., Kissel, G., and Zhou, S., PVT and Viscosity Measurements for Lloydminster-Aberfeldy and Cold Lake Blended Oil Systems, Paper SPE 79018, SPE International Thermal Operations and Heavy Oil Symposium and International Horizontal Well Technology Conference, Calgary, AB, 2002.

Fu, B.C.H., and Phillips, C.R., New Technique for Determination of Diffusivities of Volatile Hydrocarbons in Semi-solid Bitumen, Fuel, 58 (8), 557-560, 1979.

Ghez, R., Diffusion Phenomena: Cases and Studies, Kluwer Academic \& Plenum Publishers, New York, 2001.

Green, D.W., and Willhite, G.P., Enhanced Oil Recovery, Henry L. Doherty Memorial Fund of AIME, Society of Petroleum Engineers, SPE Textbook Series, Vol. 6, Richardson, TX, 1998.

Grogan, A.T., and Pinczewski, W.V., The Role of Molecular Diffusion Processes in Tertiary $\mathrm{CO}_{2}$ Flooding, J. Pet. Tech., 39 (5), 591-602, 1987.

Grogan, A.T., Pinczewski, W.V., Ruskauff, G.J., and Orr Jr, F.M., Diffusion of $\mathrm{CO}_{2}$ at Reservoir Conditions: Models and Measurements, SPE Reservoir Eng., 3 (1), 93$102,1988$.

Hall, K.R., and Yarborough, L., A New Equation of State for Z-factor Calculations, Oil \& Gas J., 71 (25), 82-92, 1973.

Hayduk, W., and Cheng, S.C., Review of Relation Between Diffusivity and Solvent Viscosity in Dilute Liquid Solutions, Chem. Eng. Sci., 26 (5), 635-646, 1971.

Hirschfelder, J.O., Curtiss, C.F., and Bird, R.B., Molecular Theory of Gases and Liquids, John Wiley \& Sons, Inc., New York, 1967. 
Hiss, T.G., and Cussler, E.L., Diffusion in High Viscosity Liquids, $A I C h E J ., 19$ (4), 859$861,1973$.

Husain, A., and Gangiah, K., Optimization Techniques for Chemical Engineers, The Macmillan Company of India Limited, Lucknow, India, 1976.

Jacobs, M.H., Diffusion Processes, Springer-Verlag New York, Inc., New York, 1967.

Jaubert, J.N., Avaullee, L., and Pierre, C., Is It Still Necessary to Measure the Minimum Miscibility Pressure?, Ind. Eng. Chem. Res., 41 (2), 303-310, 2002.

Jost, W., Diffusion in Solids, Liquids and Gases, Academic Press, New York, 1960.

Lake, L.W., Enhanced Oil Recovery, Prentice Hall, Inc., New Jersey, 1989.

Lee, B.I., and Kesler, M.G., A Generalized Thermodynamic Correlation Based on ThreeParameter Corresponding States, AIChE J., 21 (3), 510-528, 1975.

McCain, Jr., W.D., The Properties of Petroleum Fluids, Second Edition, PennWell Publishing Company, Tulsa, OK, 1990.

Mehrotra, A.K., Garg, A., and Svrcek, W.Y., Prediction of Mass Diffusivity of $\mathrm{CO}_{2}$ into Bitumen, Can. J. Chem. Eng., 65 (5), 826-832, 1987.

Mehrotra, A.K., Sarkar, M., and Svrcek, W.Y., Bitumen Density and Gas Solubility Predictions Using the Peng-Robinson Equation of State, AOSTRA J. Res., 1 (4), 215$229,1985$.

Mehrotra, A.K., and Svrcek, W.Y., Viscosity, Density and Gas Solubility Data for Oil Sand Bitumens Part III: Wabasca Bitumen Saturated with $\mathrm{N}_{2}, \mathrm{CO}, \mathrm{CO}_{2}$ and $\mathrm{C}_{2} \mathrm{H}_{6}$, AOSTRA J. Res., 2 (2), 83-93, 1985. 
Mehrotra, A.K., Svrcek, W.Y., Helper, L.G., Xu, Y., and Fu, C.T., Comments on Solubility of Carbon Dioxide in Tar Sand Bitumen: Experimental Determination and Modeling, Ind. Eng. Chem. Res., 31 (5), 1422-1423, 1992.

Miller, K.A., EOR Pilot Review - Husky Experience, Paper No. 11, Annual Technical Meeting of the South Saskatchewan Section, The Petroleum Society of CIM, Regina, SK, 1987.

Miller, K.A., Heavy Oil and Bitumen - Not Glamorous, But Often Profitable, J. Can. Pet. Tech., 33 (4), 13-15, 1994.

Miller, K.A., Moore, R.G., Ursenbach, M.G., Laureshen, C.J., and Mehta, S.A., Proposed Air Injection Recovery of Cold-Produced Heavy Oil Reservoirs, J. Can. Pet. Tech., $41(3), 40-49,2002$.

Moore, R.G., Laureshen, C.J., Belgrave, J.D.M., Ursenbach, M.G., and Mehta, S.A., In Situ Combustion: New Ideas for an Old Process, Annual Heavy Oil and Oil Sands Technology Symposium, Canadian Heavy Oil Association, Calgary, AB, 1994.

Nguyen, T.A., and Farouq Ali, S.M., Effect of Nitrogen on the Solubility and Diffusivity of Carbon Dioxide into Oil and Oil Recovery by the Immiscible WAG Process, $J$. Can. Pet. Tech., 37 (2), 24-31, 1998.

Oballa, V., and Butler, R.M., An Experimental Study of Diffusion in the BitumenToluene System, J. Can. Pet. Tech., 28 (2), 63-69, 1989.

Quail, B., Hill, G.A., and Jha, K.N., Correlations of Viscosity, Gas Solubility, and Density for Saskatchewan Heavy Oils, Ind. Eng. Chem. Res., 27 (3), 519-523, 1988. Reid, R.C., Prausnitz, J.M., and Poling, B.E., The Properties of Gases and Liquids, Fourth Edition, McGraw-Hill Companies, New York, 1987. 
Renner, T.A., Measurement and Correlation of Diffusion Coefficients for $\mathrm{CO}_{2}$ and Rich Gas Applications, SPE Reservoir Eng., 3 (2), 517-523, 1988.

Riazi, M.R., A New Method for Experimental Measurement of Diffusion Coefficients in Reservoir Fluids, J. Pet. Sci. \& Eng., 14 (3-4), 235-250, 1996.

Riazi, M.R., and Whitson, C.H., Estimating Diffusion Coefficients of Dense Fluids, Ind. Eng. Chem. Res., 32 (12), 3081-3088, 1993.

Sachs, W., The Diffusional Transport of Methane in Liquid Water: Method and Result of Experimental Investigation at Elevated Pressure, J. Pet. Sci. \& Eng., 21 (3-4), 153164, 1998.

Schmidt, T., Mass Transfer by Diffusion, AOSTRA Technical Handbook on Oil Sands, Bitumens and Heavy Oils, Alberta Oil Sands Technology and Research Authority, Edmonton, AB, 1989.

Schmidt, T., Leshchyshyn, T.H., and Puttagunta, V.R., Diffusivity of Carbon Dioxide into Athabasca Bitumen, Paper 82-33-100, Annual Technical Meeting of the Petroleum Society of CIM, Calgary, AB, 1982.

Sigmund, P.M., Prediction of Molecular Diffusion at Reservoir Conditions, Part I. Measurement and Prediction of Binary Dense Gas Diffusion Coefficients, J. Can. Pet. Tech., 15 (2), 48-57, 1976.

Sridhar, T., and Potter, O.E., Predicting Diffusion Coefficients, AIChE J., 23 (4), 590$592,1977$.

Srivastava, R.K., Huang, S.S., and Dyer, S.B., and Mourits, F.M., A Comparison of Minimum Miscibility Pressure Determinations for Weyburn $\mathrm{CO}_{2}$ Solvent Design, 
Paper 94-50, Annual Technical Meeting of the Petroleum Society of CIM, Calgary, $\mathrm{AB}, 1994$.

Standing, M.B., and Katz, D.L., Density of Natural Gases, Trans. AIME, 146, 140-149, 1942.

Strausz, O.P., Bitumen and Heavy Oil Chemistry, AOSTRA Technical Handbook on Oil Sands, Bitumens and Heavy Oils, Alberta Oil Sands Technology and Research Authority, Edmonton, AB, 1989.

Svrcek, W.Y., and Mehrotra, A.K., Gas Solubility, Viscosity and Density Measurements for Athabasca Bitumen, J. Can. Pet. Tech., 21 (4), 31-38, 1982.

Tharanivasan, A.K., Yang, C., and Gu, Y., Combined Interface Mass Transfer and Bulk Molecular Diffusion in the Measurement of Solvent Diffusivity in Heavy Oil, Recent Progress in Transport Phenomena, International Symposium on Transport Phenomena (ISTP-14), Bali, Indonesia, 2003.

Tharanivasan, A.K., Yang, C., and Gu, Y., Comparison of Three Different Interface Mass Transfer Models Used in the Experimental Measurement of Solvent Diffusivity in Heavy Oil, J. Pet. Sci. \& Eng., 44 (3-4), 269-282, 2004 a.

Tharanivasan, A.K., Yang, C., and Gu, Y., Measurements of Molecular Diffusion Coefficients of Carbon Dioxide and Methane in Heavy Oil, Paper 2004-081, Canadian International Petroleum Conference, Calgary, AB, $2004 \mathrm{~b}$.

Tharanivasan, A.K., Yang, C., and Gu, Y., Measurements of Molecular Diffusion Coefficients of Carbon Dioxide, Methane and Propane in Heavy Oil Under Reservoir Conditions, J. Can. Pet. Tech., Submitted, 2004c. 
Umesi, N.O., and Danner, R.P., Predicting Diffusion Coefficients in Nonpolar Solvents, Ind. Eng. Chem. Process Des. Dev., 20 (4), 662-665, 1981.

Upreti, S.R., Experimental Measurement of Gas Diffusivity in Bitumen: Results for $\mathrm{CO}_{2}$, $\mathrm{CH}_{4}, \mathrm{C}_{2} \mathrm{H}_{6}$ and $\mathrm{N}_{2}$, Ph.D. dissertation, Department of Chemical and Petroleum Engineering, University of Calgary, AB, 2000.

Upreti, S.R., and Mehrotra, A.K., Diffusivity of $\mathrm{CO}_{2}, \mathrm{CH}_{4}, \mathrm{C}_{2} \mathrm{H}_{6}$, and $\mathrm{N}_{2}$ in Athabasca Bitumen, Can. J. Chem. Eng., 80 (1), 116-125, 2002.

Upreti, S.R., and Mehrotra, A.K., Experimental Measurement of Gas Diffusivity in Bitumen: Results for Carbon Dioxide, Ind. Eng. Chem. Res., 39 (4), 1080-1087, 2000.

Walas, S.M., Modeling with Differential Equations in Chemical Engineering, Butterworth-Heinmann Series in Chemical Engineering, Boston, 1991.

Wang, W., and Gu, Y., Detection and Reuse of the Produced Chemicals in AlkalineSurfactant-Polymer Floods, Paper SPE 84075, SPE Annual Technical Conference and Exhibition, Denver, CO, 2003.

Wen, Y., Bryan, J., and Kantzas, A., Estimation of Diffusion Coefficients in Bitumen Solvent Mixtures as Derived from Low Field NMR Spectra, Paper 2003-017, Canadian International Petroleum Conference, Calgary, AB, 2003.

Whitson, C.H., and Brule, M.R., Phase Behavior, Monograph Vol. 20, Henry L. Doherty Series, Society of Petroleum Engineers Inc., Richardson, TX, 2000.

Wilke, C.R., and Chang, P., Correlation of Diffusion Coefficients in Dilute Solutions, AIChE J., 1 (2), 264-270, 1955. 
Wittink, D.R., The Application of Regression Analysis, Allyn and Bacon, Inc., Boston, 1988.

Yang, C., and Gu, Y., A New Method for Measuring Solvent Diffusivity in Heavy Oil by Dynamic Pendant Drop Shape Analysis (DPDSA), Paper SPE 84202, SPE Annual Technical Conference and Exhibition, Denver, CO, 2003.

Yang, D., and Gu, Y., Interfacial Tensions and Visual Interactions of Crude Oil-Brine$\mathrm{CO}_{2}$ Systems Under Reservoir Conditions, Paper 2004-083, Canadian International Petroleum Conference, Calgary, AB, 2004.

Yarborough, L., and Hall, K.R., A New Equation of State for Z-factor Calculations, Oil \& Gas J., 72 (7), 86-88, 1974.

Yazdani, J.A., and Maini, B.B., Effect of Drainage Height and Grain Size on the Convective Dispersion in the Vapex Process: Experimental Study, Paper SPE 89409, SPE/DOE Symposium on Improved Oil Recovery, Tulsa, OK, 2004.

Young, A.A., One SPE, J. Pet. Tech., 55 (7), 8-10, 2003.

$\mathrm{Yu}, \mathrm{C} . \mathrm{L} .$, The Time-Dependent Diffusion of $\mathrm{CO}_{2}$ in $n$-Hexadecane at Elevated Pressures, Master thesis, Department of Chemical and Petroleum Engineering, University of Calgary, AB, 1984.

Zhang, Y.P., Hyndman, C.L., and Maini, B.B., Measurement of Gas Diffusivity in Heavy Oils, J. Pet. Sci. \& Eng., 25 (1-2), 37-47, 2000. 


\section{Appendix A: Analytical Solutions to Diffusion Equation}

Appendix A.1 Derivation of analytical solution given in Eq. [3.10] for the equilibrium $\mathrm{BC}$

The diffusion equation together with the initial condition, the boundary condition at the bottom of the diffusion cell and the equilibrium $\mathrm{BC}$ at the solvent-heavy oil interface is expressed in terms of dimensionless variables as:

$$
\begin{array}{cc}
\frac{\partial C}{\partial \tau}=\frac{\partial^{2} C}{\partial X^{2}}, & \\
\left.C(X, \tau)\right|_{\tau=0}=0 & 0 \leq X \leq 1, \\
\left.\frac{\partial C}{\partial X}\right|_{X=0}=0 & \tau>0, \\
\left.C(X, \tau)\right|_{X=1}=1 & \tau>0 .
\end{array}
$$

The specific solution for this interface mass transfer model can be obtained by separating the desired solution, $C(X, \tau)$, into two components, namely, the steady state solution $v(X)$, and the transient state solution $\omega(X, \tau)$. Therefore, the desired solution is written as:

$$
C(X, \tau)=v(X)+\omega(X, \tau)
$$

The differential equation governing the steady state can be assumed to be:

$$
\frac{d^{2} v}{d X^{2}}=0
$$

By applying the boundary conditions, Eq. [3.8] and Eq. [3.9a]:

$$
\left.\frac{d v}{d X}\right|_{X=0}=0 \text { and }\left.\quad v(X)\right|_{X=1}=1 \text {. }
$$


Thus, the solution to Eq. [A.2] is obtained as:

$$
v(X)=1
$$

This is considered as a special solution, which represents the equilibrium solvent concentration at the steady state, i.e., when the heavy oil is completely saturated with the solvent under the equilibrium pressure, $P_{\text {eq }}$.

By substituting Eq. [A.1] into Eq. [3.6] and using Eq. [A.2], the differential equation for describing the transient state and its initial and boundary conditions are given by:

$$
\begin{gathered}
\frac{\partial \omega}{\partial \tau}=\frac{\partial^{2} \omega}{\partial X^{2}}, \\
\left.\omega(X, \tau)\right|_{\tau=0}=\left.C(X, \tau)\right|_{\tau=0}-v(X)=-v(X)=-1, \\
\left.\frac{\partial \omega}{\partial X}\right|_{X=0}=\left.\frac{\partial C}{\partial X}\right|_{X=0}-\left.\frac{d v}{d X}\right|_{X=0}=0, \\
\left.\omega(X, \tau)\right|_{X=1}=\left.C(X, \tau)\right|_{X=1}-\left.v(X)\right|_{X=1}=0 .
\end{gathered}
$$

Thus, the IC and BCs become homogeneous so that Eq. [A.4] can be solved by using the separation of variables method. The solution can be assumed to be:

$$
\omega(X, \tau)=q(\tau) p(X)
$$

where the concentration $\omega(X, \tau)$ is equal to a product of two functions, one is a function of $\tau$ only, and the other is a function of $X$ only. Substituting Eq. [A.8] into Eq. [A.4] gives:

$$
\frac{1}{q} \frac{d q}{d \tau}=\frac{1}{p} \frac{d^{2} p}{d X^{2}} .
$$

It is noted from Eq. [A.9] that the L.H.S is a function of $\tau$ only, whereas the R.H.S is a 
function of $X$ only. Thus, both the L.H.S and R.H.S should be equal to a constant, $k$, which is independent of both $\tau$ and $X$,

$$
\frac{d q}{d \tau}=k q, \text { and } \frac{d^{2} p}{d X^{2}}=k p
$$

Here, there are three possible cases, namely, $k$ is positive, negative and zero. When $k$ is positive and zero, it can be shown that the transient solution to Eq. [A.4] together with its initial and boundary conditions in Eqs. [A.5-7] has to be zero. Therefore, the negative value of $k$ is of interest, neglecting the other two cases. When $k$ is negative, $k=-\lambda^{2}$, Equation [A.10] is written as:

$$
\frac{d q}{d \tau}=-\lambda^{2} q, \text { and } \frac{d^{2} p}{d X^{2}}=-\lambda^{2} p
$$

The analytical solutions to the above ordinary differential equations are obtained as:

$$
q(\tau)=R e^{-\lambda^{2} \tau}, \text { and } p(X)=M^{\prime} \cos (\lambda X)+N^{\prime} \sin (\lambda X),
$$

where $R, M^{\prime}$ and $N^{\prime}$ are arbitrary constants. Thus Equation [A.8] can be written as:

$$
\omega(X, \tau)=e^{-\lambda^{2} \tau}[M \cos (\lambda X)+N \sin (\lambda X)]
$$

where $M=R M^{\prime}$ and $N=R N^{\prime}$. In Eq. [A.12], the arbitrary constant $R$ is eliminated by incorporating it into the arbitrary constants, $M$ and $N$. Differentiating Eq. [A.12] with respect to $X$ yields:

$$
\frac{\partial \omega}{\partial X}=\lambda e^{-\lambda^{2} \tau}[-M \sin (\lambda X)+N \cos (\lambda X)]
$$

By applying the boundary conditions in Eqs. [A.6-7], Equations [A.12-13] become:

$$
\left.\omega(X, \tau)\right|_{X=1}=\left.e^{-\lambda^{2} \tau}[M \cos (\lambda X)+N \sin (\lambda X)]\right|_{X=1}=M(\cos \lambda) e^{-\lambda^{2} \tau}=0
$$

and

$$
\left.\frac{\partial \omega}{\partial X}\right|_{X=0}=\left.\lambda e^{-\lambda^{2} \tau}[-M \sin (\lambda X)+N \cos (\lambda X)]\right|_{X=0}=N \lambda e^{-\lambda^{2} \tau}=0 .
$$


This means that $N=0$ and $\cos \lambda=0(M \neq 0)$, which result in an infinite number of nontrivial solutions. Hence, $\lambda_{n}=\frac{(2 n+1) \pi}{2}, n=0,1,2,3, \ldots$, and Equation [A.12] becomes:

$$
\omega(X, \tau)=M \cos \left[\frac{(2 n+1) \pi}{2} X\right] e^{-\frac{(2 n+1)^{2} \pi^{2}}{4} \tau},
$$

where the infinite number of eigen values and their corresponding eigen functions are:

$$
\begin{array}{ll}
n=0, \lambda_{0}=\frac{\pi}{2}, & \omega_{0}(X, \tau)=M_{0} \cos \left[\frac{\pi}{2} X\right] e^{-\frac{\pi^{2}}{4} \tau}, \\
n=1, \lambda_{1}=\frac{3 \pi}{2}, & \omega_{1}(X, \tau)=M_{1} \cos \left[\frac{3 \pi}{2} X\right] e^{-\frac{9 \pi^{2}}{4} \tau}, \\
\cdot & \\
n=n, \lambda_{n}=\frac{(2 n+1) \pi}{2}, \quad \omega_{n}(X, \tau)=M_{n} \cos \left[\frac{(2 n+1) \pi}{2} X\right] e^{-\frac{(2 n+1)^{2} \pi^{2}}{4} \tau} .
\end{array}
$$

Then by using the principle of superposition, all the eigen values and eigen functions are combined to obtain the general solution for $\omega(X, \tau)$ :

$$
\omega(X, \tau)=\sum_{n=0}^{\infty} M_{n} \cos \left[\frac{(2 n+1) \pi}{2} X\right] e^{-\frac{(2 n+1)^{2} \pi^{2}}{4} \tau}
$$

By applying the initial condition in Eq. [A.5], Eq. [A.14] gives:

$$
\omega(X, 0)=\sum_{n=0}^{\infty} M_{n} \cos \left[\frac{(2 n+1) \pi}{2} X\right]=-1 \text {. }
$$

Here, the orthonormal property of cosine function in the Fourier series can be used to obtain the value of $M_{n}$ by multiplying $\cos \left[\frac{(2 m+1) \pi}{2} X\right]$ on both sides of Eq. [A.15] and integrating the resultant equation from $X=0$ to $X=1$. If $m=n$, one can obtain: 


$$
M_{n}=-\frac{2(-1)^{n}}{\frac{(2 n+1) \pi}{2}} \text {, }
$$

and Eq. [A.14] becomes:

$$
\omega(X, \tau)=-2 \sum_{n=0}^{\infty}(-1)^{n} \frac{\cos \left[\frac{(2 n+1) \pi}{2} X\right]}{\frac{(2 n+1) \pi}{2}} e^{-\frac{(2 n+1)^{2} \pi^{2}}{4} \tau}
$$

By substituting Eq. [A.3] and Eq. [A.16] in Eq. [A.1], the final solution to the dimensionless form of diffusion equation in Eq. [3.6] is obtained:

$$
C(X, \tau)=1-2 \sum_{n=0}^{\infty}(-1)^{n} \frac{\cos \left[\frac{(2 n+1) \pi}{2} X\right]}{\frac{(2 n+1) \pi}{2}} e^{-\frac{(2 n+1)^{2} \pi^{2}}{4} \tau}
$$

Appendix A.2 Derivation of analytical solution given in Eq. [3.15] for the nonequilibrium $\mathrm{BC}$

The diffusion equation together with the initial condition, boundary condition at the bottom of the diffusion cell and the non-equilibrium BC at the solvent-heavy oil interface is expressed in terms of dimensionless variables as:

$$
\begin{array}{cc}
\frac{\partial C}{\partial \tau}=\frac{\partial^{2} C}{\partial X^{2}}, & 0 \leq X \leq 1, \\
\left.C(X, \tau)\right|_{\tau=0}=0 & \tau>0, \\
\left.\frac{\partial C}{\partial X}\right|_{X=0}=0 & \tau>0 . \\
\left.\frac{\partial C}{\partial X}\right|_{X=1}=k_{D}\left[1-\left.C(X, \tau)\right|_{X=1}\right] &
\end{array}
$$


In order to solve the diffusion equation by the separation of variables method, the nonhomogeneous boundary condition can be transformed into the homogeneous boundary condition by variable transformation:

$$
\omega(X, \tau)=1-C(X, \tau)
$$

Therefore, Equations [3.6-3.8] and Equation [3.9c] can be rewritten in terms of $\omega$ :

$$
\begin{array}{cc}
\frac{\partial \omega}{\partial \tau}=\frac{\partial^{2} \omega}{\partial X^{2}}, & \\
\left.\omega(X, \tau)\right|_{\tau=0}=1 & 0 \leq X \leq 1, \\
\left.\frac{\partial \omega}{\partial X}\right|_{X=0}=0 & \tau>0, \\
\left.\frac{\partial \omega}{\partial X}\right|_{X=1}=-k_{D}\left[\left.\omega(X, \tau)\right|_{X=1}\right] & \tau>0 .
\end{array}
$$

The solution to Eq. [A.18] can be assumed to be:

$$
\omega(X, \tau)=q(\tau) p(X)
$$

where the concentration $\omega(X, \tau)$ is a product of two functions, one is a function of $\tau$ only and the other is a function of $X$ only. Substituting Eq. [A.22] in Eq. [A.18] gives:

$$
\frac{1}{q} \frac{d q}{d \tau}=\frac{1}{p} \frac{d^{2} p}{d X^{2}} .
$$

It is noted from Eq. [A.23] that the L.H.S is a function of $\tau$ only, whereas the R.H.S is a function of $X$ only. Thus, both the L.H.S and R.H.S should be equal to a constant, $k$, which is independent of both $\tau$ and $X$,

$$
\frac{d q}{d \tau}=k q, \text { and } \frac{d^{2} p}{d X^{2}}=k p
$$


Here, three cases are possible, namely, $k$ is positive, negative and zero. When $k$ is positive and zero, it can be shown that the transient solution to Eq. [A.18] together with the initial and boundary conditions in Eqs. [A.19-21] has to be zero. Therefore, the negative value of $k$ is of interest, ignoring the other two cases. When $k$ is negative, $k=-\lambda^{2}$, Equation [A.24] is written as:

$$
\frac{d q}{d \tau}=-\lambda^{2} q, \text { and } \frac{d^{2} p}{d X^{2}}=-\lambda^{2} p
$$

The respective analytical solutions to the ordinary differential equations in Eq. [A.25] are obtained as:

$$
q(\tau)=R e^{-\lambda^{2} \tau}, \text { and } p(X)=M^{\prime} \cos (\lambda X)+N^{\prime} \sin (\lambda X)
$$

where $R, M^{\prime}$ and $N^{\prime}$ are arbitrary constants. Thus Equation [A.22] becomes:

$$
\omega(X, \tau)=e^{-\lambda^{2} \tau}[M \cos (\lambda X)+N \sin (\lambda X)]
$$

where $M=R M^{\prime}$ and $N=R N^{\prime}$. In Eq. [A.26], the arbitrary constant $R$ is omitted incorporating it into the arbitrary constants, $M$ and $N$. Differentiating Eq. [A.26] with respect to $X$ yields:

$$
\frac{\partial \omega}{\partial X}=\lambda e^{-\lambda^{2} \tau}[-M \sin (\lambda X)+N \cos (\lambda X)]
$$

Applying the boundary conditions, Eqs. [A.20-21], in Eqs. [A.26-27] gives:

$$
\begin{gathered}
N \lambda e^{-\lambda^{2} t}=0, \quad \Rightarrow N=0, \\
\tan \lambda=\frac{k_{D}}{\lambda} .
\end{gathered}
$$

and

Therefore, by using the principle of superposition, Equation [A.26] becomes:

$$
\omega(X, \tau)=\sum_{n=1}^{\infty} M_{n} \cos \left(\lambda_{n} X\right) e^{-\lambda_{n}^{2} \tau}
$$


By applying the initial condition in Eq. [A.19], Equation [A.29] should be equal to:

$$
\omega(X, 0)=\sum_{n=1}^{\infty} M_{n} \cos \lambda_{n} X=1 \text {. }
$$

Here, the orthonormal property of cosine function in the Fourier series can be used to obtain the value of $M_{n}$ by multiplying $\cos \left(\lambda_{m} X\right)$ on both sides of Eq. [A.30] and integrating the resultant equation from $X=0$ to $X=1$. By assuming $\lambda_{m}=\lambda_{n}$ and using the identity:

$$
\int_{0}^{1} \cos \left(\lambda_{m} X\right) \cos \left(\lambda_{n} X\right) d X= \begin{cases}0 & \text { when } \lambda_{m} \neq \lambda_{n} \\ \frac{1}{2}\left(1+\frac{1}{2 \lambda_{n}} \sin 2 \lambda_{n}\right) & \text { when } \lambda_{m}=\lambda_{n}\end{cases}
$$

one can obtain:

$$
M_{n}=\frac{2 \sin \lambda_{n}}{\left(\lambda_{n}+\sin \lambda_{n} \cos \lambda_{n}\right)}
$$

Substituting Eq. [A.31] in Eq. [A.29] yields:

$$
\omega(X, \tau)=2 \sum_{n=1}^{\infty} \frac{\sin \lambda_{n}}{\left(\lambda_{n}+\sin \lambda_{n} \cos \lambda_{n}\right)} \cos \left(\lambda_{n} X\right) e^{-\lambda_{n}^{2} \tau}
$$

By means of the variable transformation in Eq. [A. 17], Equation [A.32] becomes:

$$
C(X, \tau)=1-2 \sum_{n=1}^{\infty} \frac{\sin \lambda_{n}}{\left(\lambda_{n}+\sin \lambda_{n} \cos \lambda_{n}\right)} \cos \left(\lambda_{n} X\right) e^{-\lambda_{n}^{2} \tau}
$$




\section{Appendix B: Calculation of Compressibility Factor}

Appendix B.1 Lee-Kesler correlation

The Z-factor in the equation of state (EOS) for a real gas is calculated by using the Lee-Kesler correlation (Lee and Kesler, 1975):

$$
Z=Z^{(o)}+\omega\left(Z^{(r)}-Z^{(o)}\right) / \omega^{(r)}
$$

In this equation, both the compressibility factor for simple fluid, $Z^{(o)}$, and compressibility factor for reference fluid, $Z^{(r)}$, are represented by the following reduced form of modified Bendict-Webb-Rubin (BWR) equation of state (Edmister and Lee, 1984).

$$
Z=\left(\frac{P_{r} V_{r}}{T_{r}}\right)=1+\frac{B}{V_{r}}+\frac{C}{V_{r}^{2}}+\frac{D}{V_{r}^{5}}+\frac{c_{4}}{T_{r}^{3} V_{r}^{2}}\left(\beta+\frac{\gamma}{V_{r}^{2}}\right) e^{\left(-\frac{\gamma}{V_{r}^{2}}\right)}
$$

where

$$
\begin{gathered}
V_{r}=\frac{V P_{c}}{R T_{c}}, \\
B=b_{1}-\frac{b_{2}}{T_{r}}-\frac{b_{3}}{T_{r}^{2}}-\frac{b_{4}}{T_{r}^{3}}, \\
C=c_{1}-\frac{c_{2}}{T_{r}}+\frac{c_{3}}{T_{r}^{3}}, \\
D=d_{1}+\frac{d_{2}}{T_{r}} .
\end{gathered}
$$

In Eqs. [B.1-6], $P, T, V$, subscripts $c$ and $r$, and $\omega$ denote the pressure, temperature, volume, the value at the critical condition, the reduced value and the acentric factor, respectively. The molecular weights, critical properties and acentric factors of the test solvents in this thesis study, namely carbon dioxide, methane and propane, are given in Table B.1. In addition, the constants used in Eqs. [B.2-B.6] for a simple fluid $(\omega=0)$ and 
the reference fluid $\left(\omega^{(r)}=0.3978\right.$ for $\mathrm{n}$-octane) are given in Table B.2. A FORTRAN subroutine program for the calculation of compressibility factor by using the abovedescribed Lee-Kesler correlation is given in Appendix C.5.

Table B.1 Properties of pure solvents used in this study (Edmister and Lee, 1984)

\begin{tabular}{|c|c|c|c|c|}
\hline Solvent & $\begin{array}{c}\text { Molecular weight } \\
M[\mathrm{~g} / \mathrm{mol}]\end{array}$ & $\begin{array}{c}\text { Critical pressure } \\
P_{c}[\mathrm{kPa}]\end{array}$ & $\begin{array}{c}\text { Critical temperature } \\
T_{c}\left[{ }^{\circ} \mathrm{C}\right]\end{array}$ & $\begin{array}{c}\text { Acentric factor } \\
\omega\end{array}$ \\
\hline $\mathrm{CO}_{2}$ & 44.0095 & 7384 & 31.06 & 0.2250 \\
\hline $\mathrm{CH}_{4}$ & 16.0425 & 4604 & -82.5 & 0.0115 \\
\hline $\mathrm{C}_{3} \mathrm{H}_{8}$ & 44.0956 & 4249 & 96.7 & 0.1454 \\
\hline
\end{tabular}

Table B.2 Constants used in Equations [B.2-B.6] (Edmister and Lee, 1984)

\begin{tabular}{|c|c|c|}
\hline Constants & $\begin{array}{c}\text { Simple fluids } \\
(\omega=0)\end{array}$ & $\begin{array}{c}\text { Reference fluid } \\
\left(\omega^{(r)}=0.3978 \text { for n-octane }\right)\end{array}$ \\
\hline$b_{1}$ & 0.1181193 & 0.2026579 \\
\hline$b_{2}$ & 0.265728 & 0.331511 \\
\hline$b_{3}$ & 0.154790 & 0.027655 \\
\hline$b_{4}$ & 0.030323 & 0.203488 \\
\hline$c_{1}$ & 0.0236744 & 0.0313385 \\
\hline$c_{2}$ & 0.0186984 & 0.0503618 \\
\hline$c_{3}$ & 0.0 & 0.016901 \\
\hline$c_{4}$ & 0.042724 & 0.041577 \\
\hline$d_{1} \times 10^{4}$ & 0.155488 & 0.48736 \\
\hline$d_{2} \times 10^{4}$ & 0.623689 & 0.0740336 \\
\hline$\beta$ & 0.65392 & 1.226 \\
\hline$\gamma$ & 0.060167 & 0.03754 \\
\hline
\end{tabular}


Appendix B.2 Hall-Yarborough equation

The Z-factor in the EOS can also be calculated by using the Hall-Yarborough equation (Dake, 1978):

$$
Z=\frac{0.06125 p_{p r} \bar{t} e^{-1.2(1-\bar{t})^{2}}}{y}
$$

where $p_{p r}$ is the pseudo reduced pressure $\left(P / P_{\mathrm{pc}}\right) ; P_{\mathrm{pc}}$ is the pseudo critical pressure; $\bar{t}$ is the reciprocal of the pseudo reduced temperature $\left(T_{\mathrm{pc}} / T\right) ; T_{\mathrm{pc}}$ is the pseudo critical temperature; and $y$ is the "reduced" density which can be obtained by solving the following non-linear equation:

$$
\begin{aligned}
& -0.06125 p_{p r} \bar{t} \mathrm{e}^{-1.2(1-\bar{t})^{2}}+\frac{y+y^{2}+y^{3}-y^{4}}{(1-y)^{3}}-\left(14.76 \bar{t}-9.76 \bar{t}^{2}+4.58 \bar{t}^{3}\right) y^{2} \\
& +\left(90.7 \bar{t}-242.2 \bar{t}^{2}+42.4 \bar{t}^{3}\right) y^{(2.18+2.82 \bar{t})}=0 .
\end{aligned}
$$

Here, the "reduced" density $y$ is defined as the product of van der Waals co-volume and density (Hall and Yarborough, 1973). A FORTRAN subroutine program for the calculation of compressibility factor by using the Hall-Yarborough equation is given in Appendix C.6. 


\section{APPENDIX C: COMPUTER PROGRAMS}

Appendix C.1 FORTRAN program for the determination of the equilibrium pressure from the measured solubility data

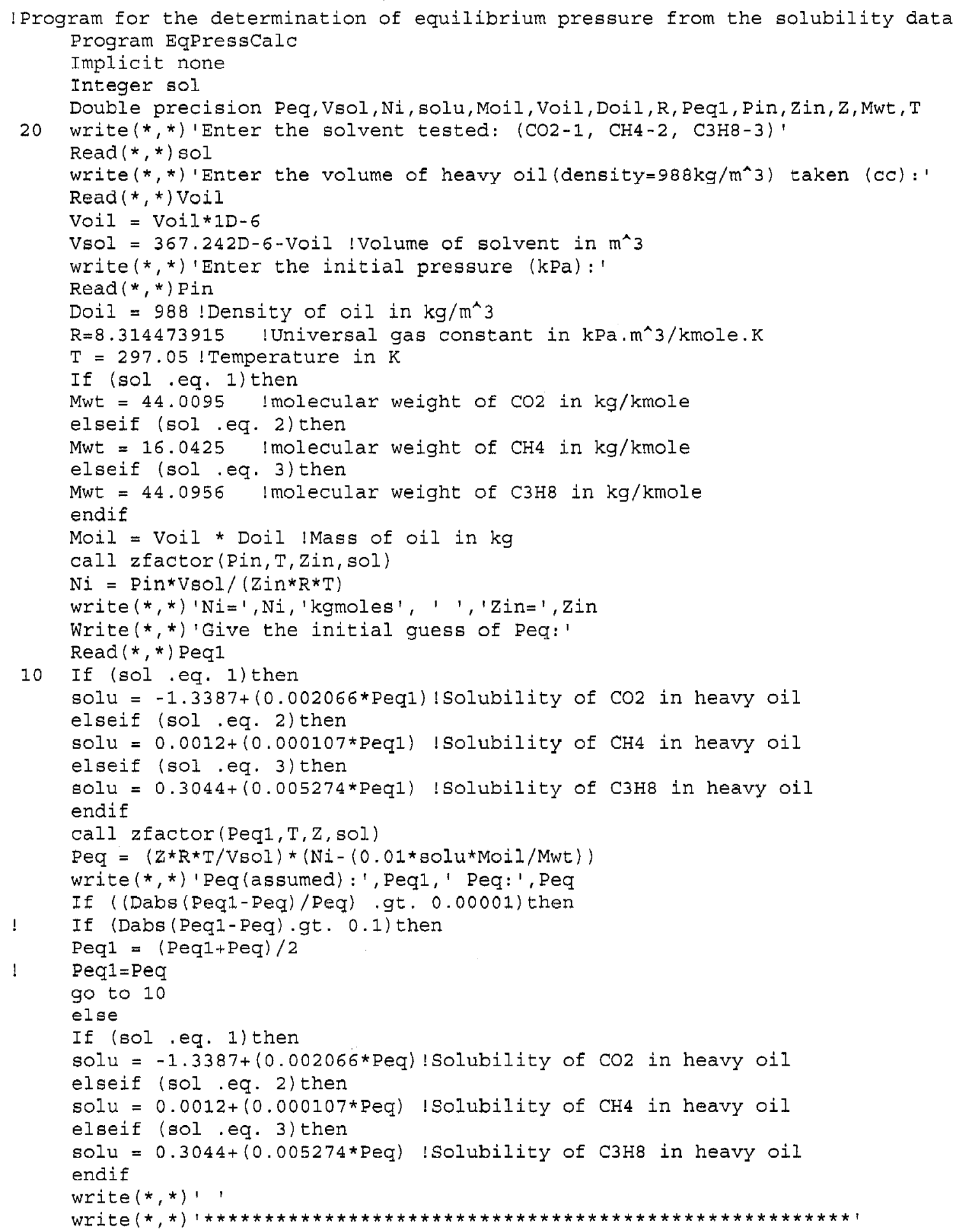


write $(*, 30)$ Peq

write $(*, 40)$ solu

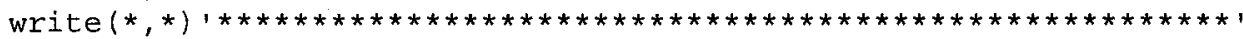

write $(*, *) '$ '

endif

go to 20

30 Format ('Equilibrium Pressure, Peq $=1, \mathrm{~F} 7.2,2 \mathrm{X}, 1,1,1 \mathrm{kPa}$ ')

40 Format ('Solubility@ Peq $=$ ',2X,F7.4,' ','g solvent/100 g hoil')

stop

end

Appendix C.2 FORTRAN program for the diffusivity determination by using the equilibrium $\mathrm{BC}$ at the solvent-heavy oil interface

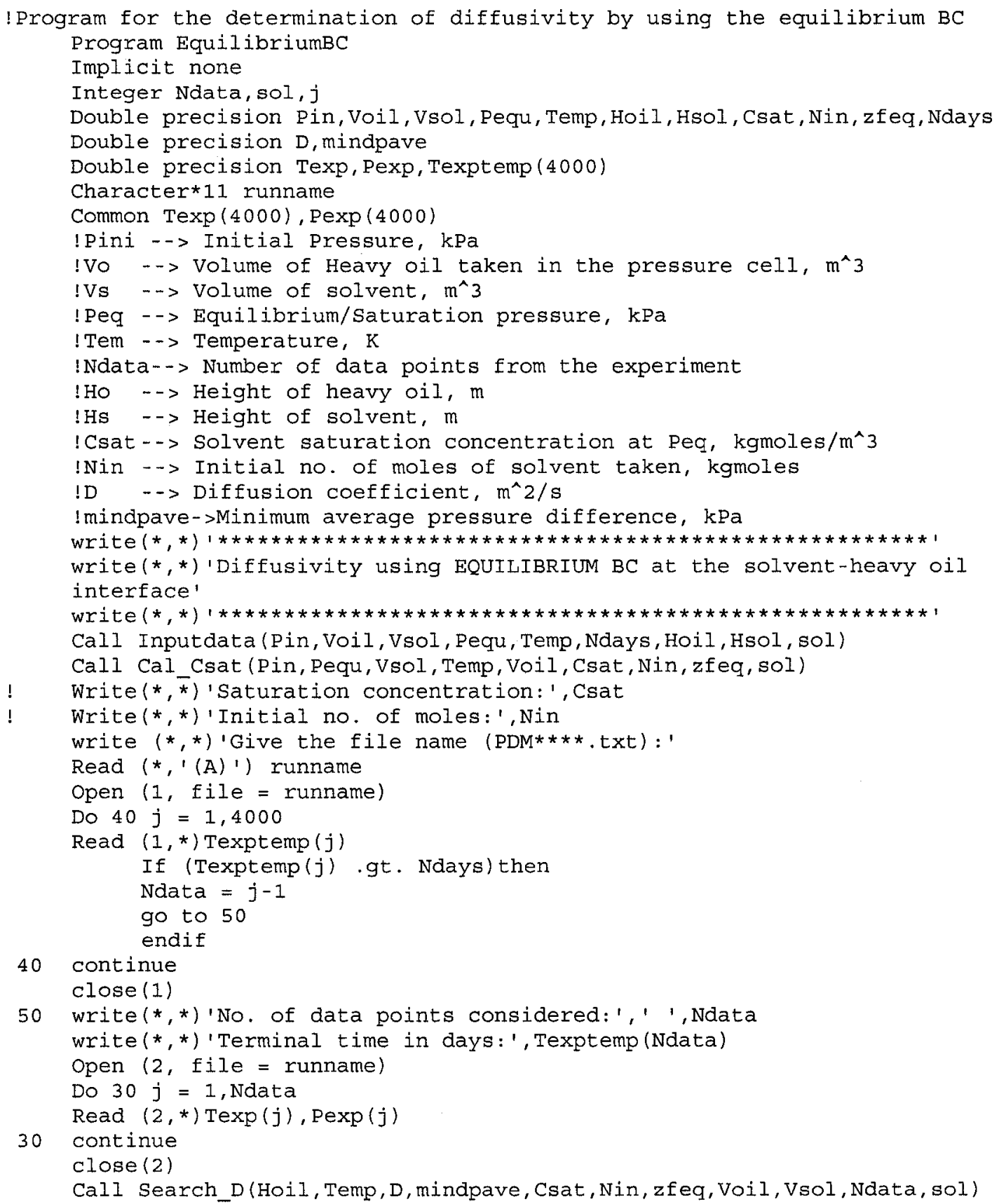




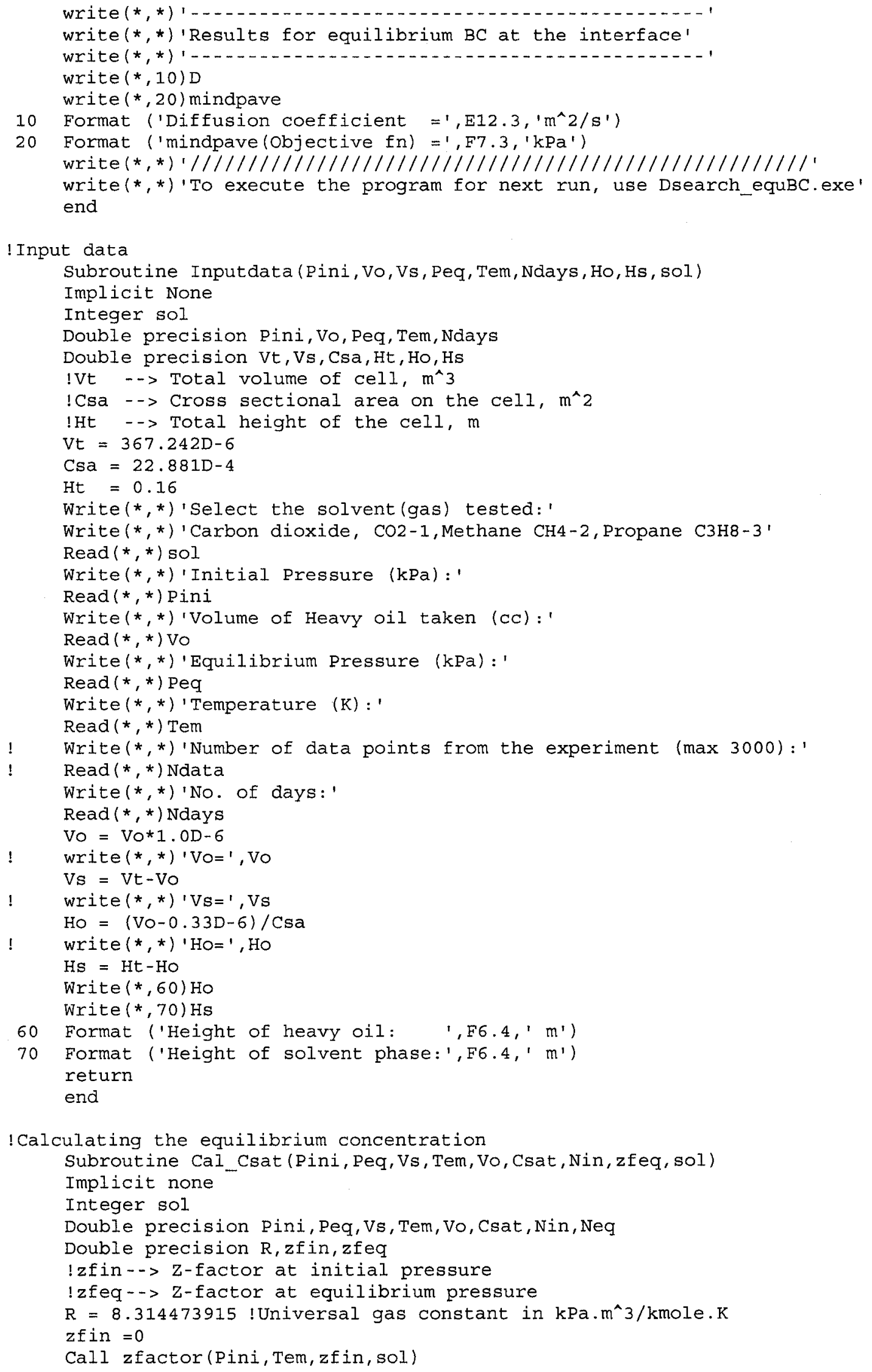


Nin $=\operatorname{Pini} * V s /(z f i n * R * T e m)$

Write $(*, *)$ 'Nin=' , Nin, 'Pin=', Pini, 'zfin=', zfin

Call zfactor (Peq, Tem, zfeq, sol)

Neq $=$ Peq $*$ Vs $/(z f e q * R * T e m)$

Write $(*, *)$ 'Neq=' , Neq, 'Peq=' , Peq, ' zfeq=' , zfeq

Csat $=($ Nin-Neq $) /$ Vo

Write $(*, *)^{\prime} \mathrm{Ceq}={ }^{\prime}$, Csat, ' kmoles $/ \mathrm{m}^{\wedge} 3$ '

Return

end.

!Searching for $D$

Subroutine Search_D(Ho, Tem, D, mindpave, Csat, Nin, zfeq, Vho, Vs, Ndata, sol)

Implicit none

Integer Ndata, sol

double precision Ho, Tem, D, mindpave, Csat, Nin, avgpd, Vho, Vs, zfeq

double precision D1,D2, delD, S, valD

double precision Texp, Pexp

Common Texp (4000), Pexp (4000)

$\mathrm{D} 1=0.010 \mathrm{D}-9$

$\mathrm{D} 2=100.01 \mathrm{D}-9$

$20 S=1000000$

If $(\mathrm{DI}$. It . 0) $\mathrm{D} 1=0.01 \mathrm{D}-9$

write (*, *) 'D1=', D1, 'D2=', D2

$\operatorname{del} D=(D 2-D 1) / 10$

write $(*, *)$ 'delD $=$ ', delD

Do $30 \mathrm{D}=\mathrm{D} 1, \mathrm{D} 2, \operatorname{del} \mathrm{D}$

Call min_obj (Ho, Tem, D, avgpd, Csat,Nin, zfeq, Vho,Vs,Ndata, sol)

If (avgpo . It. S) then

$S=$ avgpd

$\operatorname{valD}=\mathrm{D}$

Endif

30 continue

$\mathrm{D} 1=\mathrm{valD}-\mathrm{delD}$

$\mathrm{D} 2=\operatorname{valD}+$ delD

If (Dabs (D1-D2) .1t. 0.01D-9) then

$\mathrm{valD}=(\mathrm{D} 1+\mathrm{D} 2) / 2.0$

Call min_obj (Ho, Tem, D, avgpd, Csat,Nin, zfeq, Vho,Vs, Ndata, sol) go to 40

else

go to 20

endif

$40 \mathrm{D}=\mathrm{valD}$

mindpave $=\mathrm{S}$

Return

end

!Evaluating the objective function

Subroutine min_obj (Ho, Temperature,Di, avpd, Ceq, Nin, zfeq,Vo,Vs, Ndata, sol)

Implicit none

Double precision Texp, Pexp

Double precision Ho, Temperature, Di, avgpd, Ceq, Nin, avpd, zfeq

Integer $i, j, n, N d a t a, s i g, N P, N s e r i e s$, sol

Double precision $\mathrm{R}, \mathrm{Pi}$

Parameter $(\mathrm{R}=8.314473915, \mathrm{Pi}=3.1415926535$, Nseries $=1000)$

Double precision tow $(1: 4000)$, conc1, term1, term2, conc, totconc $(1: 210,1: 4000)$

Double precision area, area1, avconc (1:4000), Noil, Vo, Nre, Hs

Double precision pres,Vs, zcorr,pres1, Pressure (1:4000)

Real $\mathrm{X}$, error $(1: 4000)$, error 1

Common Texp (4000), Pexp (4000)

Do $11 \mathrm{j}=1$, Ndata

tow $(j)=T \exp (j) * D i * 24 * 3600 /($ Ho**2)!t measured in seconds

do $12 i=0,200$

$x=i * 0.005$ 


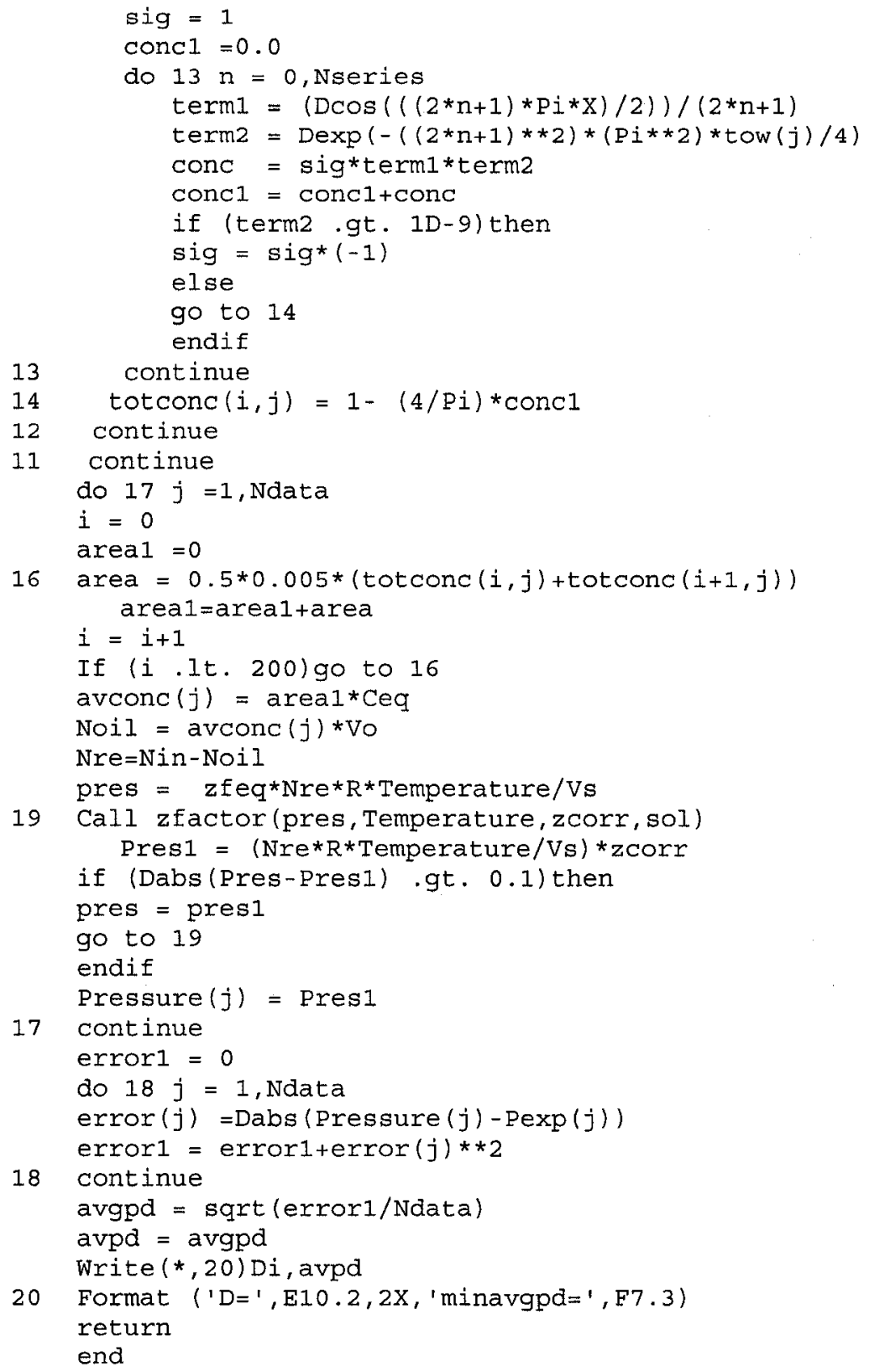

Appendix C.3 FORTRAN program for the diffusivity determination by using the quasiequilibrium $\mathrm{BC}$ at the solvent-heavy oil interface

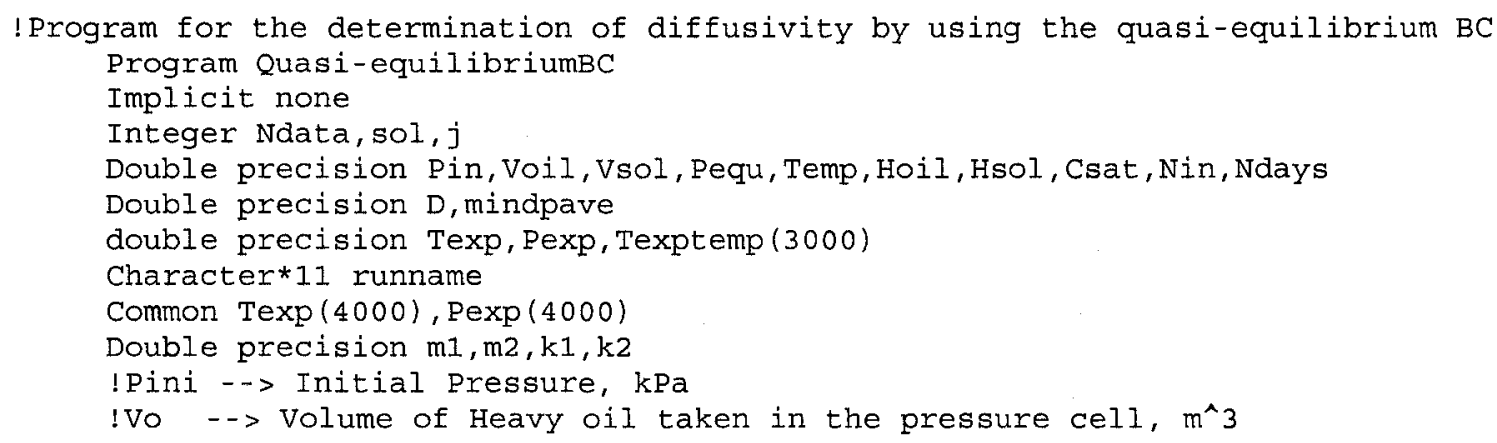




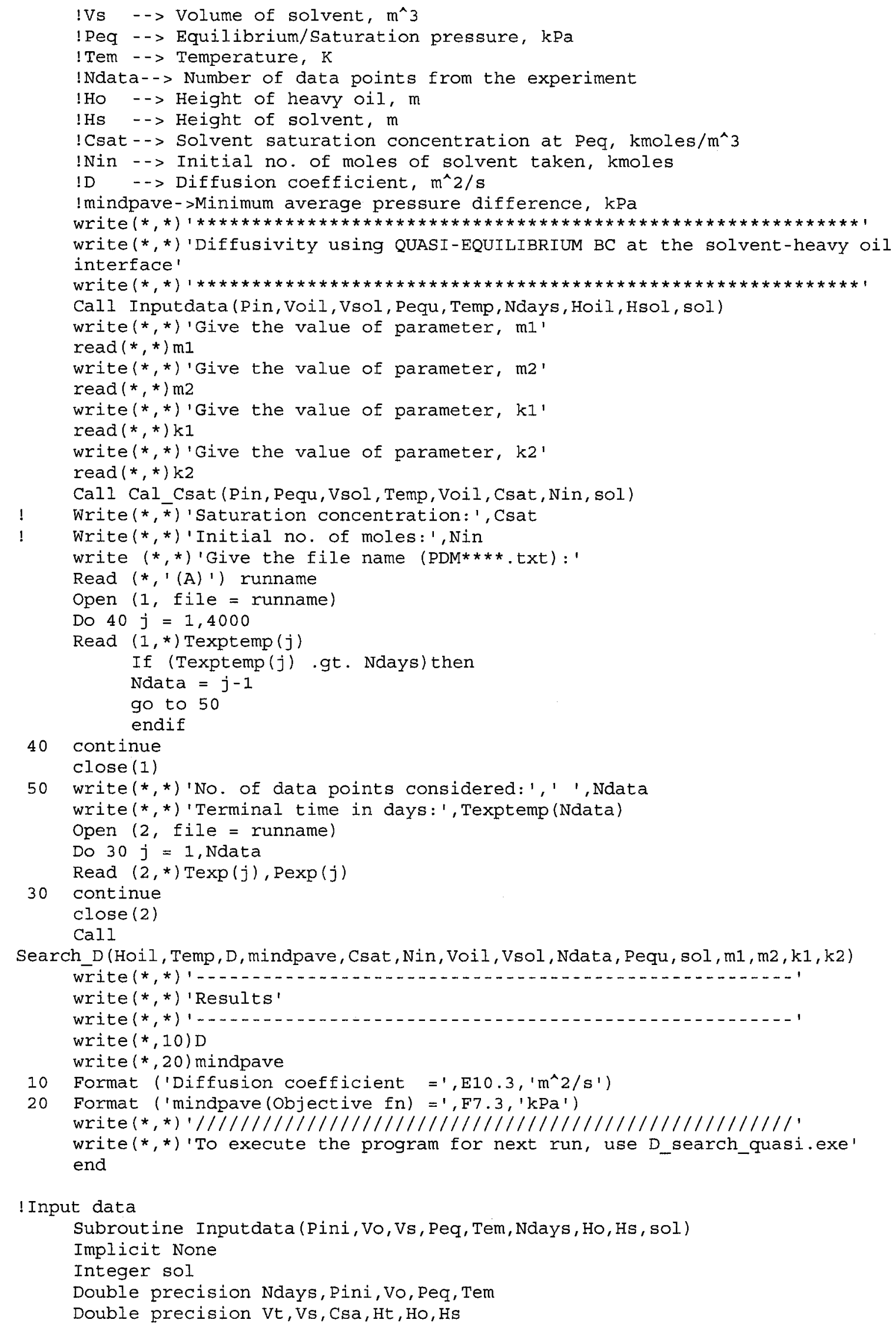




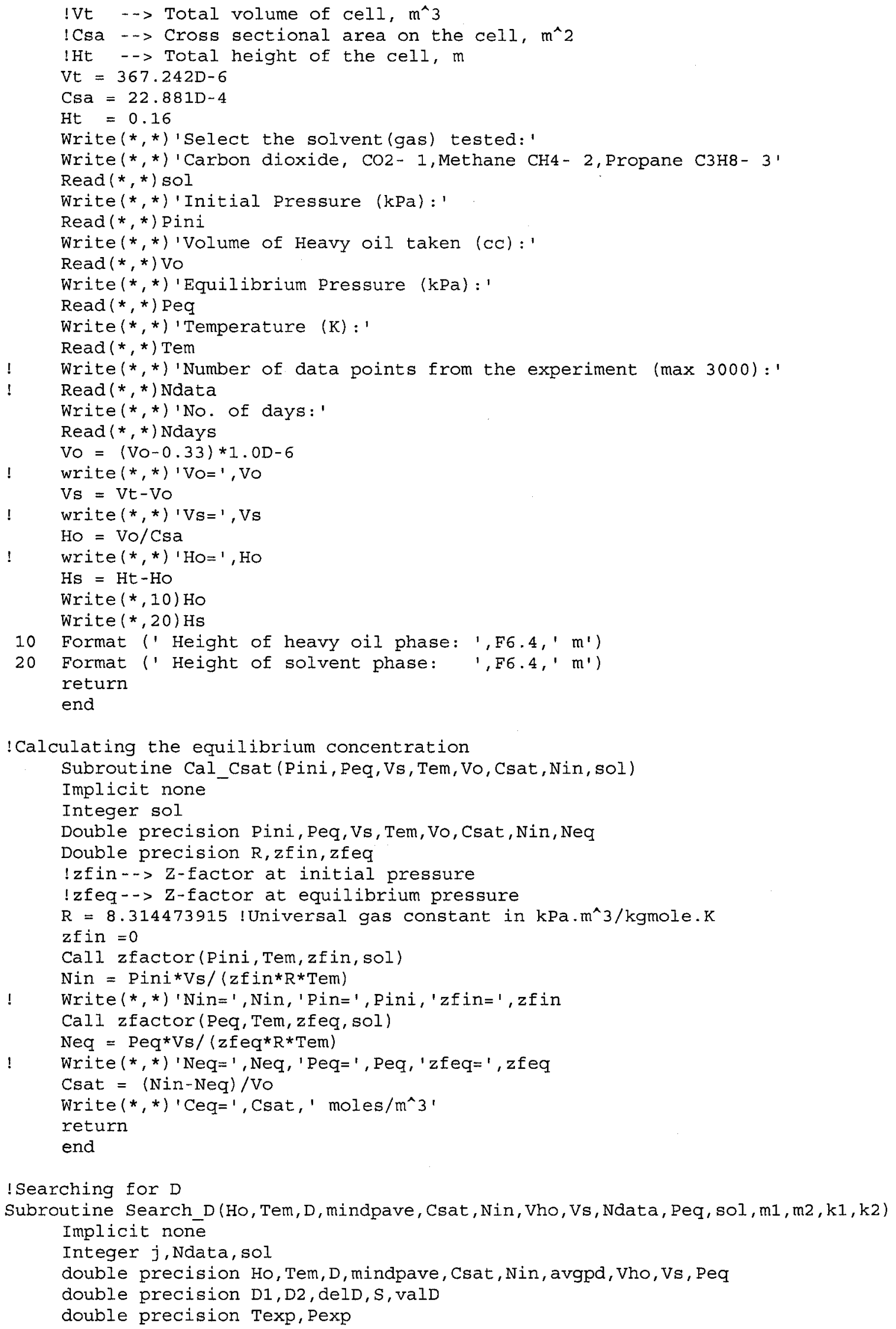


Double precision $\mathrm{m} 1, \mathrm{~m} 2, \mathrm{k} 1, \mathrm{k} 2$

Common Texp (4000), $\operatorname{Pexp}(4000)$

$\mathrm{D} 1=0.010 \mathrm{D}-9$

$\mathrm{D} 2=100.01 \mathrm{D}-9$

$20 \mathrm{~S}=1000000$

If $(\mathrm{DI}$. It . 0) DI $=0.01 \mathrm{D}-9$

write (*, *) ' $\mathrm{D} 1=$ ', $\mathrm{D} 1, \mathrm{D}^{\mathrm{D}}=$ ', $\mathrm{D} 2$

delD $=(\mathrm{D} 2-\mathrm{D} 1) / 10$

write (*,*)' del $D=$ ', delD

Do $30 \mathrm{D}=\mathrm{D} 1, \mathrm{D} 2$, delD

Call min obj (Ho, Tem, D, avgpd, Csat, Nin, Vho, Vs, Ndata, Peq, sol, m1, m2, k1, k2)

If (avgp $\bar{d}$. It. S) then

$S=$ avgpd

$\operatorname{valD}=\mathrm{D}$

endif

30 continue

$\mathrm{DI}=\operatorname{valD}-\mathrm{delD}$

$\mathrm{D} 2=\operatorname{valD}+\operatorname{delD}$

If (Dabs (D1-D2) . . . 0.01D-9) then

$\operatorname{valD}=(\mathrm{D} 1+\mathrm{D} 2) / 2.0$

Call min obj (Ho, Tem, D, avgpd, Csat, Nin, Vho, Vs, Ndata, Peq, sol, m1, m2, k1, k2) go to 40

else

go to 20

endif

$40 \mathrm{D}=\mathrm{valD}$

mindpave $=S$

return

end

IEvaluating the objective function

Subroutine

min obj (Ho, Temperature, Di , avgpd, Ceq, Nin, Vo, Vs, Ndata, Peq, sol, ml, m2 , k1, k2)

Implicit none

Double precision Ho, Temperature, Di, avgpd, Ceq, Nin, Peq

Integer $i, j, n$, Nata, Nseries, sig, NP, sol

Double precision $\mathrm{R}, \mathrm{Pi}$

Parameter $(R=8.314473915, P i=3.1415926535$, Nseries $=1000)$

Double precision tow $(1: 4000), \operatorname{conc} 1, \operatorname{term} 1, \operatorname{term} 2, \operatorname{conc}, \operatorname{tot} \operatorname{conc}(1: 210,1: 4000)$

Double precision term, cst, expo, term3, temp

Double precision alpha, beta, ka1, ka2, m1, m2, k1, k2

Double precision area, areal, avconc (1:4000), Noil, vo, Nre, Hs

Double precision pres, Vs, zcorr, presl, Pressure (1:4000)

Real $\mathrm{X}$, error $(1: 4000)$, error1

double precision Texp, Pexp

Common $\mathrm{T} \exp (4000), \mathrm{Pexp}(4000)$

alpha $=\mathrm{m} 1 /$ Peq

beta $=\mathrm{m} 2 / \mathrm{Peq}$

! write (*,*) 'alpha=', alpha, 'beta=', beta

$20 \mathrm{kal}=\mathrm{kl} * 3600 * 24 * \mathrm{Di} /\left(\mathrm{HO}_{\mathrm{O}} * 2\right)$

$\mathrm{ka} 2=\mathrm{k} 2 * 3600 * 24 * \mathrm{Di} /\left(\mathrm{HO}^{*} * 2\right)$

do $11 j=1$, Ndata

tow $(j)=\mathrm{Texp}(j) * \mathrm{Di} * 24 * 3600 /(\mathrm{Ho} * 2)$

do $12 i=0,200$

$\mathrm{X}=\mathrm{i} * 0.005$

$\operatorname{sig}=1$

conc $=0$.

$\operatorname{conc} 1=0$

If ( $i$.eq. 200 . and. $j$.ne. 1) then

conc1 $=1+(a l p h a * \exp (-\operatorname{tow}(j) / \mathrm{ka} 1))+($ beta*exp $(-$ tow $(j) / k a 2))$ else

do $13 n=0$, Nseries

term $=\operatorname{sig} * P i *(2 * n+1) * D \cos ((2 * n+1) * P i * X / 2)$ 


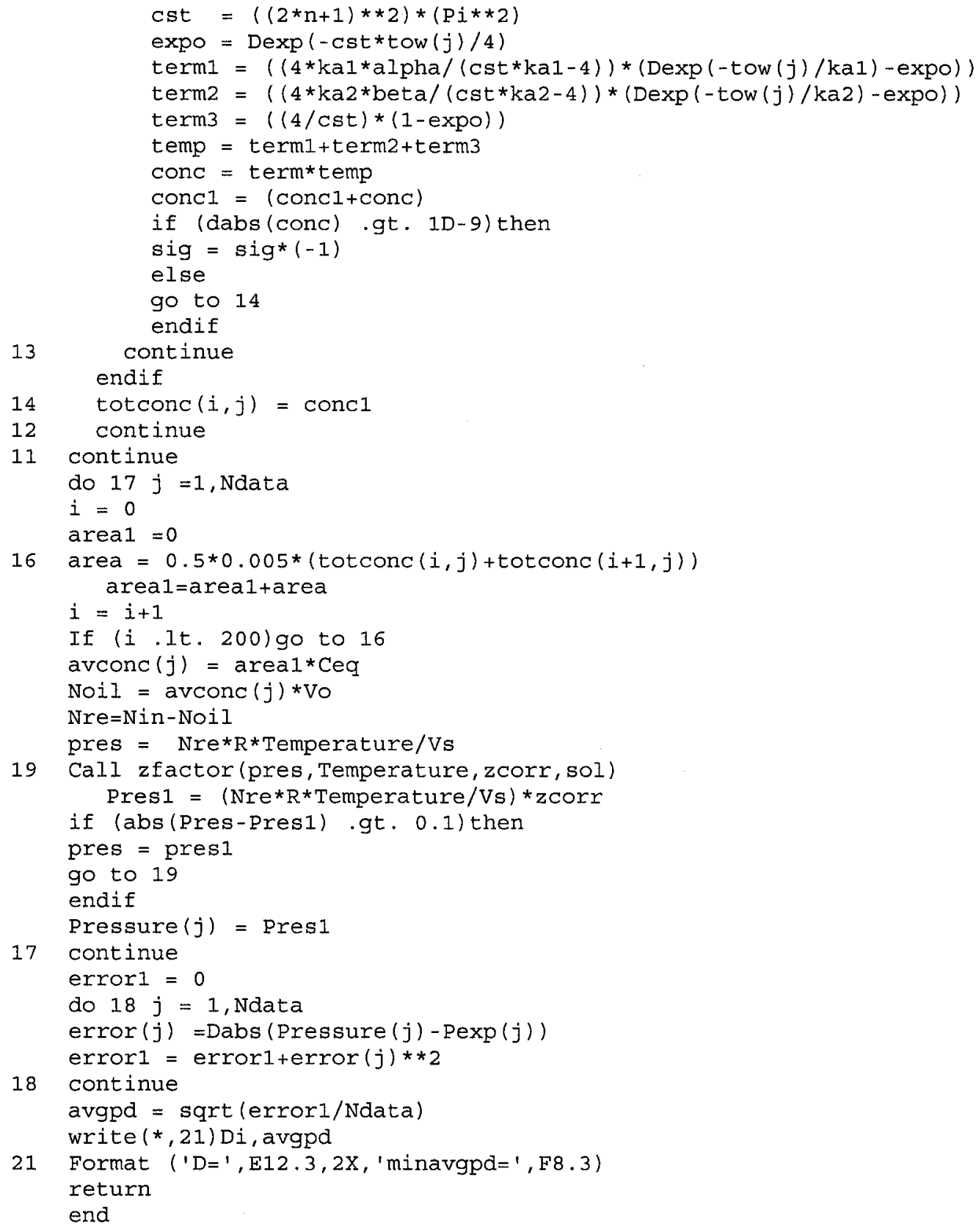

Appendix C.4 FORTRAN program for the diffusivity determination by using the nonequilibrium $\mathrm{BC}$ at the solvent-heavy oil interface

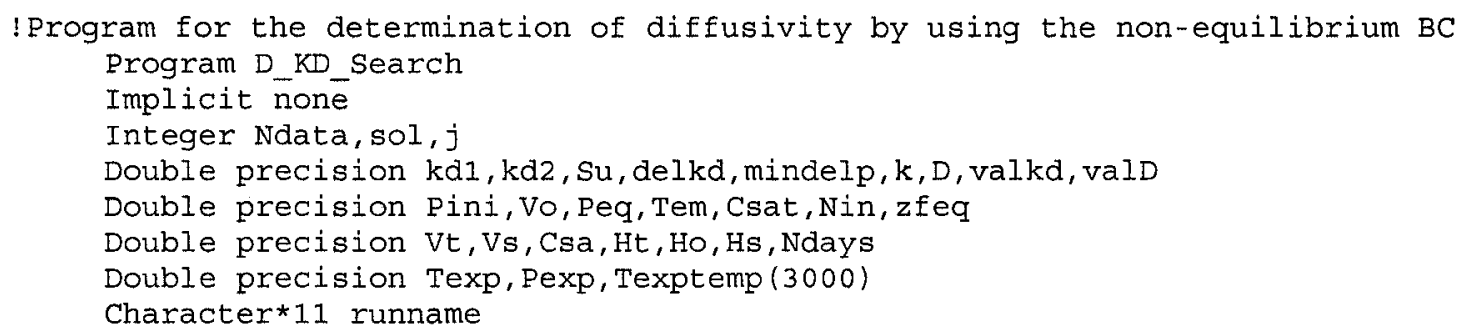




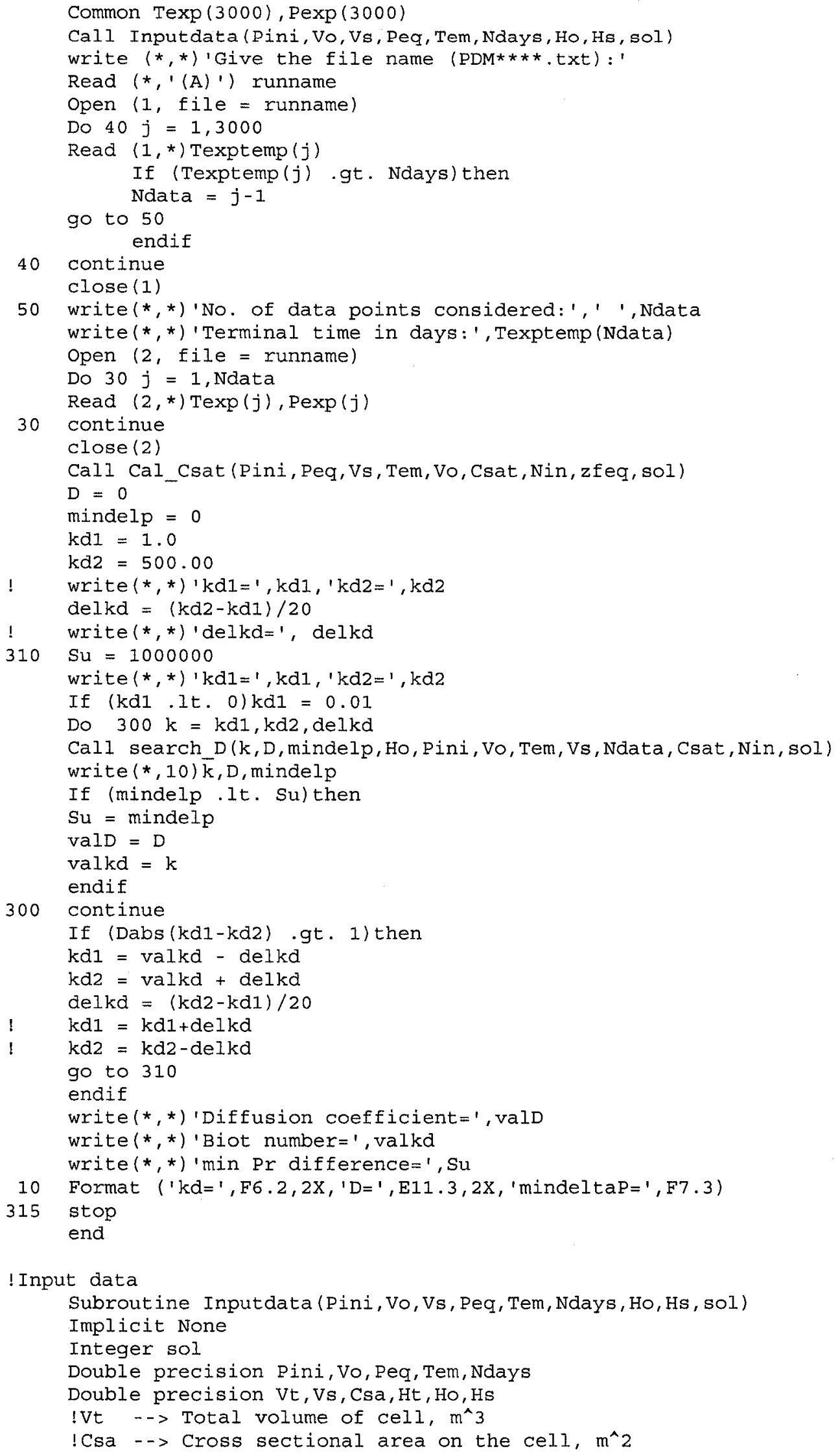




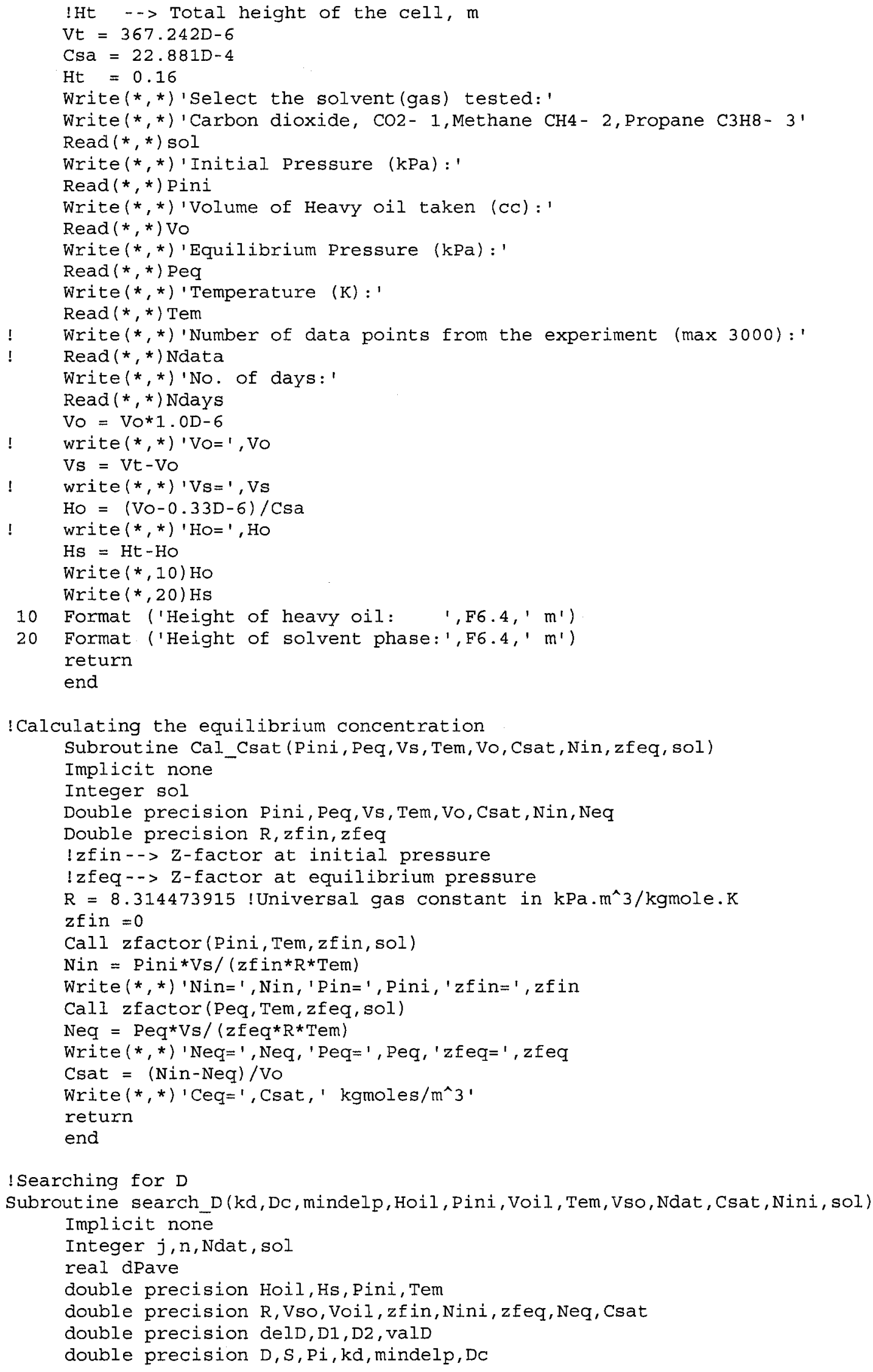


double precision Texp, Pexp

Common Texp (3000), Pexp (3000)

$\mathrm{D} 1=0.010 \mathrm{D}-9$

$\mathrm{D} 2=100 \cdot 01 \mathrm{D}-9$

100

$S=100000$

If (D1 . It. 0) D1=0.01D-9

write $(*, *) ' D 1=$ ', $D 1, D^{\prime} 2=$ ', D2

$\operatorname{del} \mathrm{D}=(\mathrm{D} 2-\mathrm{D} 1) / 10$

! write (*,*)'delD $=$ ', delD

Do $110 \mathrm{D}=\mathrm{D} 1, \mathrm{D} 2$, delD

Call min_obj (Hoil, Csat, Pini, Voil, Nini, Tem, Vso, D, dPave, Ndat, kd, sol)

If (dPave . It. S) then

$S$ = dPave

$\operatorname{valD}=\mathrm{D}$

endif

110 continue

$D 1=$ valD - delD

$\mathrm{D} 2=\mathrm{valD}+\mathrm{delD}$

If (Dabs (D1-D2) .It. 0.01D-9) then

$\operatorname{valD}=(\mathrm{D} 1+\mathrm{D} 2) / 2.0$

Call min obj (Hoil, Csat, Pini, Voil, Nini, Tem, Vso, valD, dPave, Ndat, kd, sol)

else go to $15 \overline{0}$

endif

go to 100

write $(*, *) ' m i n i m u m=', s$, 'valD $=$ ', vald

write $(*, *)$ 'diffusion coefficient in the range'

write $(*, *) ' D 1=', D 1$, to' $^{\prime}, \mathrm{D} 2={ }^{\prime}, \mathrm{D} 2$

$150 \mathrm{DC}=\mathrm{valD}$

mindelp $=$ dPave

return

end

!Evaluating the objective function

Subroutine min_obj (Ho, Ceq, Pin, Vo, Nin, Temperature, Vs, Di, avpd, Ndata, kd, sol)

Implicit none

double Precision Ho, Ceq, Pin, Vo, Nin, Temperature, Vs, R, Pi

integer $i, j, n$, Ndata, Nseries, sig, sol

parameter $(\mathrm{R}=8.314473915, \mathrm{Bi}=3.1415926535$, Nseries $=1000)$

double precision tow $(1: 3000)$, term $1, \operatorname{term} 2, \operatorname{conc}, \operatorname{conc} 1, \operatorname{tot} \operatorname{conc}(1: 210,1: 3000)$

double precision area, areal, avconc $(1: 3000), C$, Noil, pres, Pressure $(1: 3000)$

double precision Nre, zcorr, zcorrl, pres1, Di

real $x$, error $(1: 3000)$, error 1 , avgpd, avpd

double precision $k d, x 1, x 2, l a m, l a m b(1:$ Nseries), term ( $1:$ Nseries)

double precision Texp, Pexp

Common Texp (3000), Pexp (3000)

1 write $(*, *)$ 'value of $\mathrm{kd}$ ', $\mathrm{kd}$

do $21 \mathrm{n}=1$, Nseries

$x 1=(n-1) * P i$

$\mathrm{x} 2=((\mathrm{n}-1) * \mathrm{P} i)+(0.5 * \mathrm{P} i)-(1 \mathrm{D}-8)$

call eigenvalue $(x 1, x 2, k d, l a m)$

$1 \mathrm{amb}(\mathrm{n})=1 \mathrm{am}$

$\operatorname{term}(n)=(d \sin (\operatorname{lamb}(n))) /((2 * l a m b(n))+(d \sin (2 * 1 a m b(n))))$

$1 \quad$ write $(*, *) n, \operatorname{lamb}(n), \operatorname{term}(n)$

21 continue

20 do $11 j=1$, Ndata

tow $(j)=\mathrm{T} \exp (j) * \mathrm{Di} * 24 * 3600 /(\mathrm{HO} * 2)$

do $12 i=0,200$

$\mathrm{x}=i * 0.005$

conc $1=0$

do $13 \mathrm{n}=1$, Nseries

term $1=\operatorname{dcos}(1 \mathrm{amb}(\mathrm{n}) * \mathrm{x})$

term2 $=\operatorname{dexp}(-((\operatorname{Iamb}(n)) * * 2) * \operatorname{tow}(j))$ 


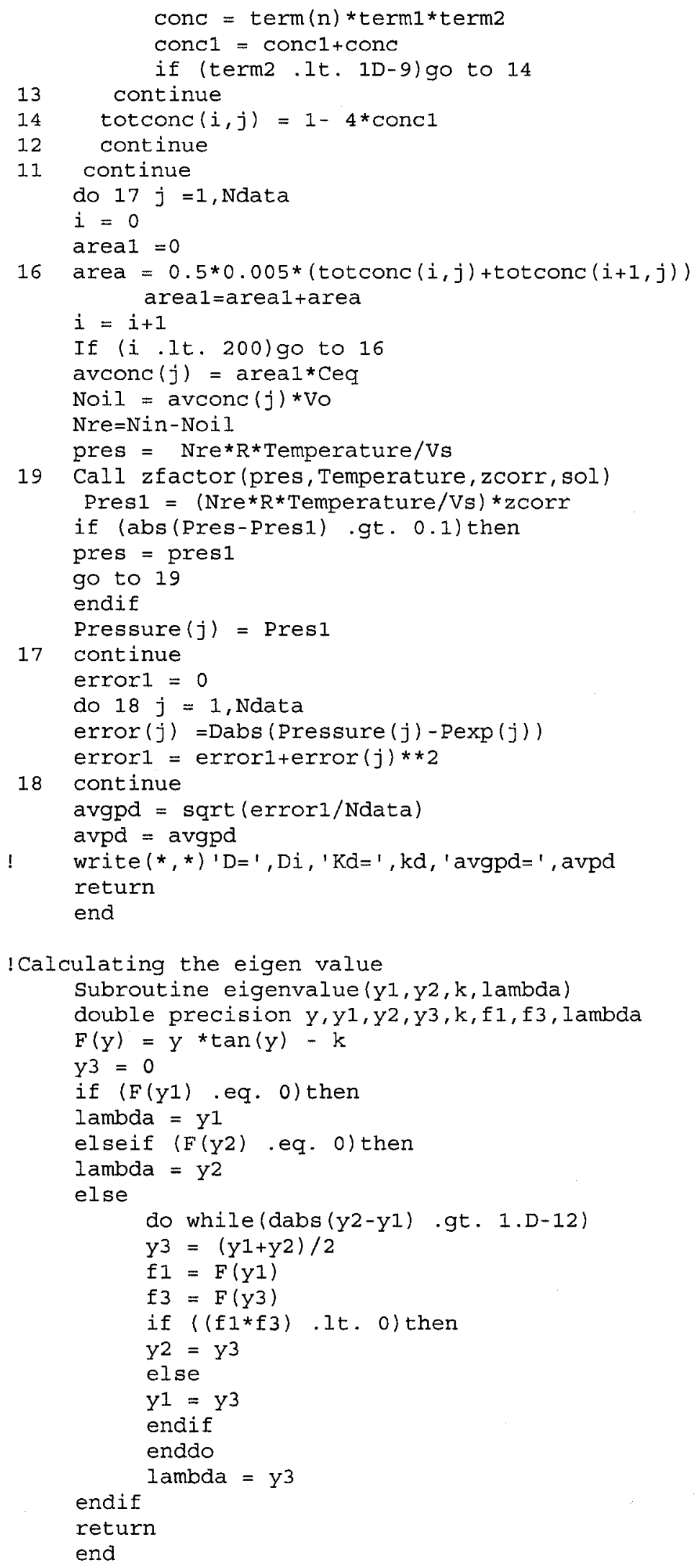


Appendix C.5 FORTRAN subroutine program for the calculation of compressibility factor by using the Lee-Kesler correlation

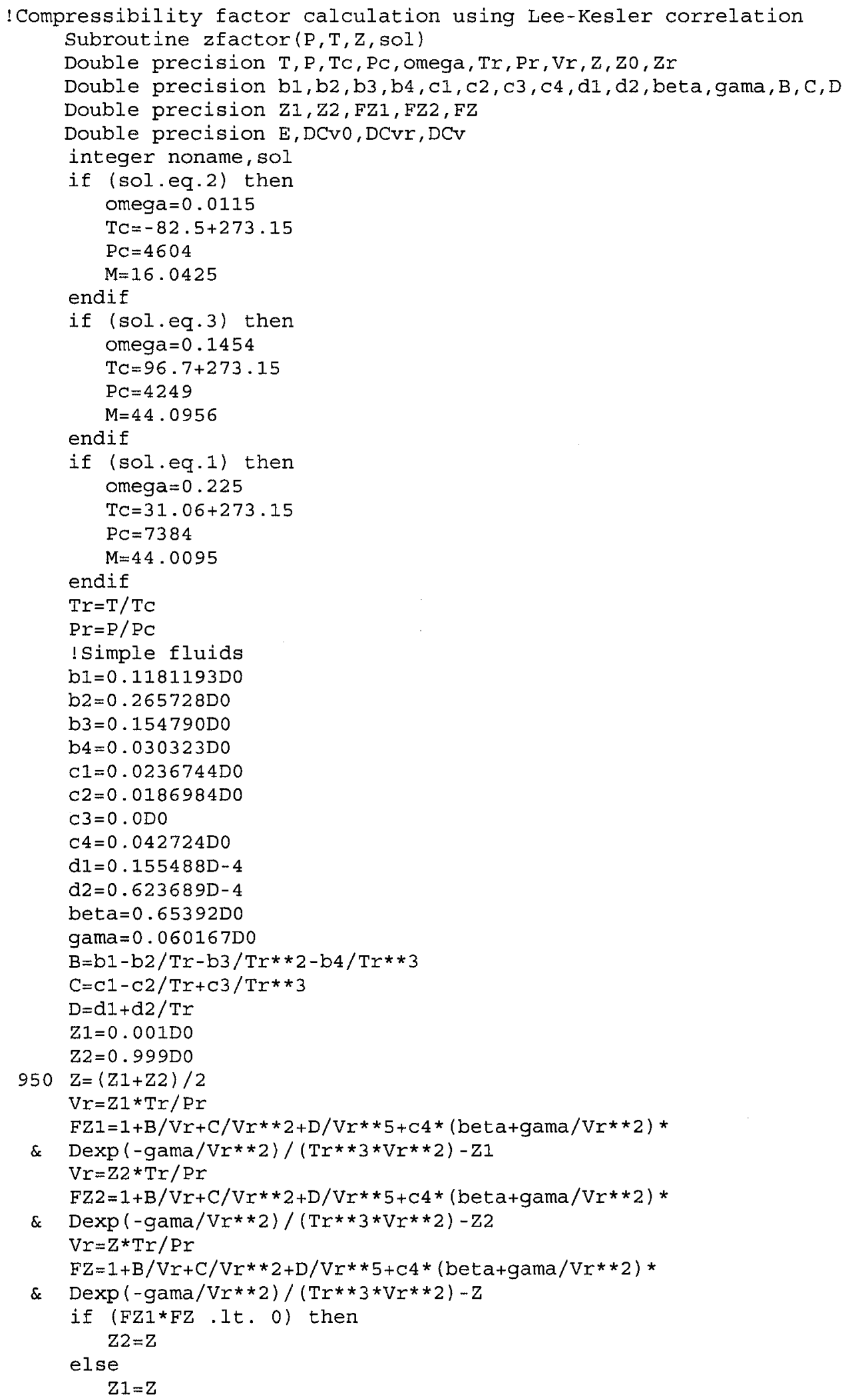




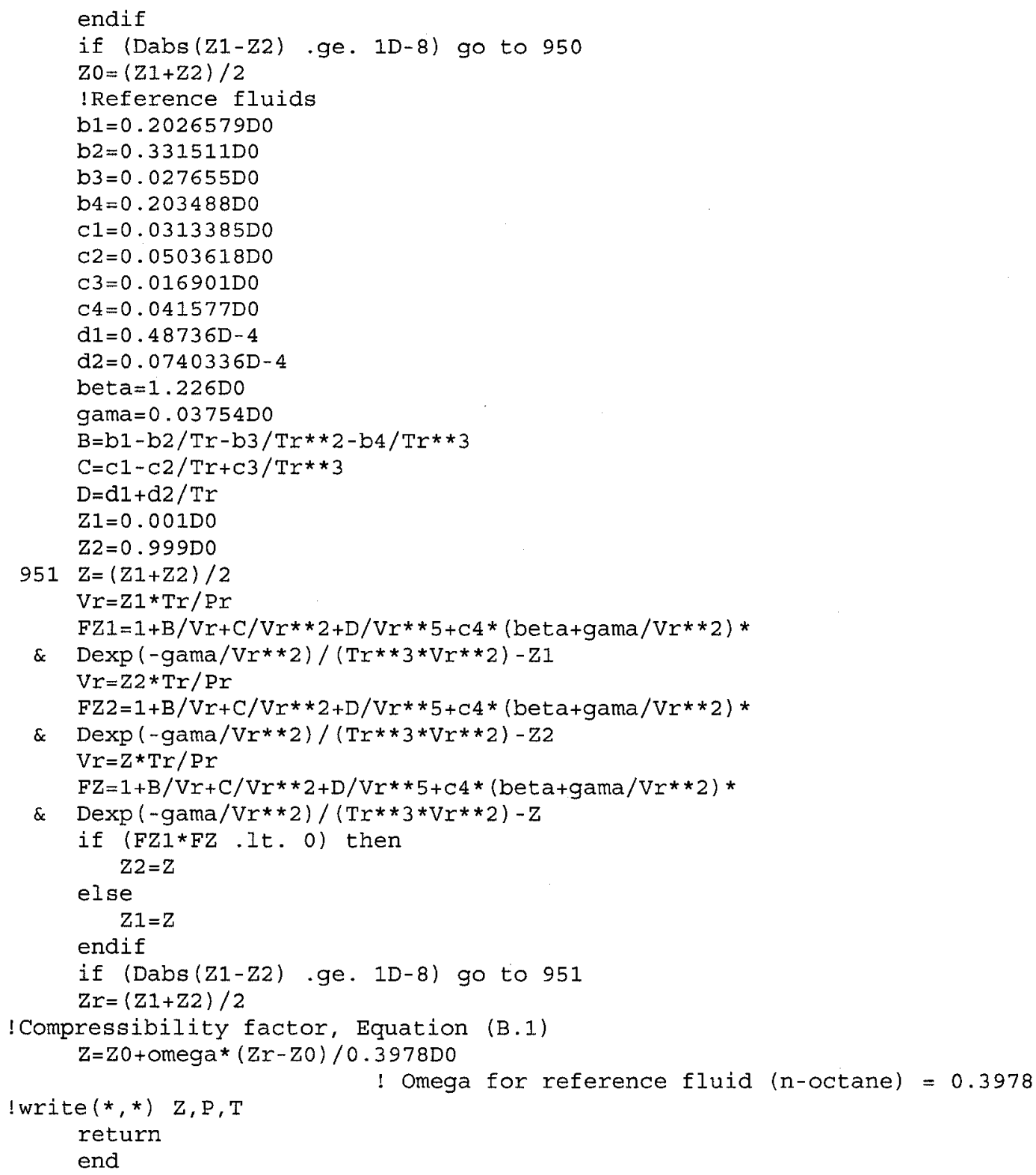

Appendix C.6 FORTRAN subroutine program for the calculation of compressibility factor by using the Hall-Yarborough equation

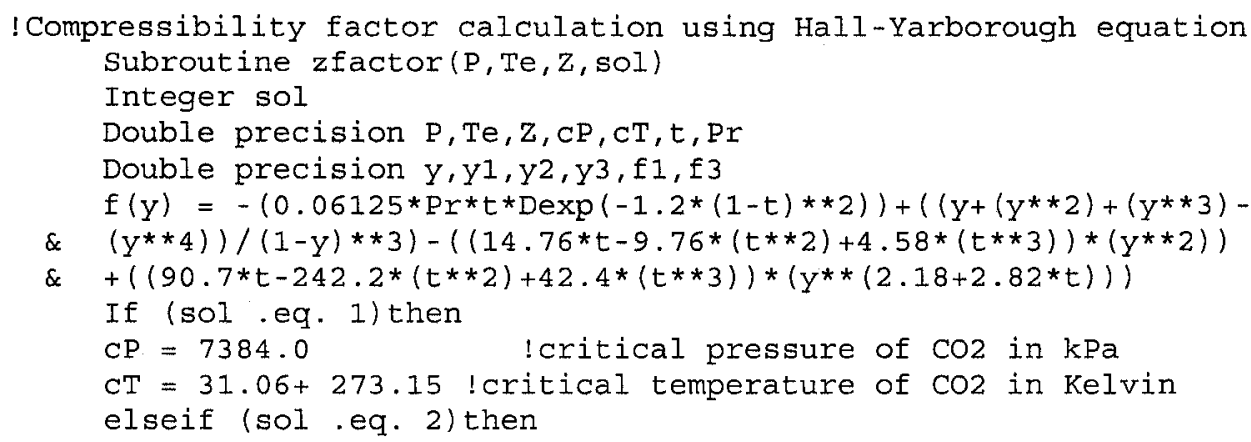




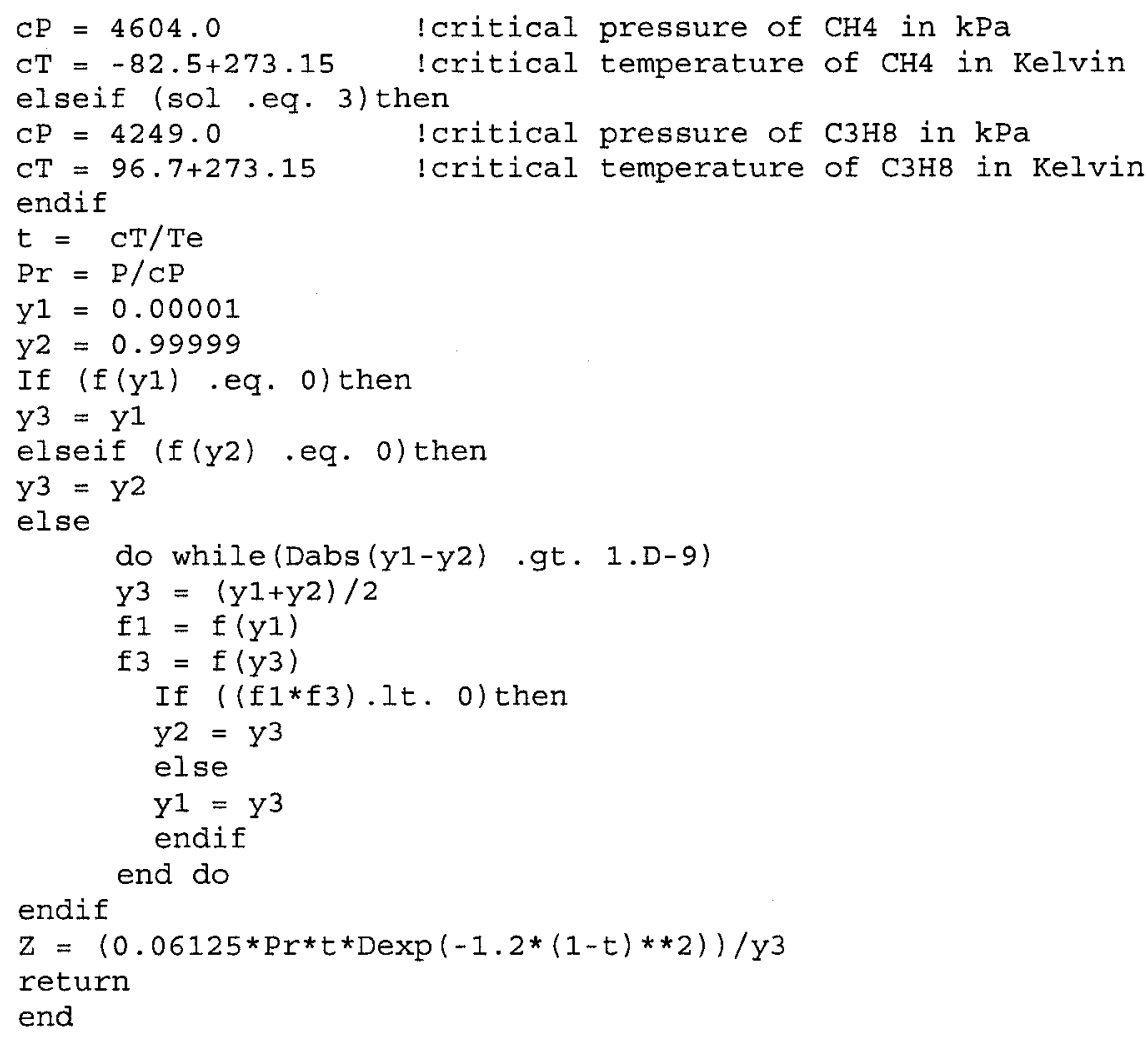

